\title{
Confirmation of TDM Capabilities in Modeling Compartmentalized WAG EOR
}

\author{
Anthony William Morrow \\ West Virginia University, awmorrow@mix.wvu.edu
}

Follow this and additional works at: https://researchrepository.wvu.edu/etd

Part of the Engineering Commons

\section{Recommended Citation}

Morrow, Anthony William, "Confirmation of TDM Capabilities in Modeling Compartmentalized WAG EOR" (2020). Graduate Theses, Dissertations, and Problem Reports. 7570.

https://researchrepository.wvu.edu/etd/7570

This Thesis is protected by copyright and/or related rights. It has been brought to you by the The Research Repository @ WVU with permission from the rights-holder(s). You are free to use this Thesis in any way that is permitted by the copyright and related rights legislation that applies to your use. For other uses you must obtain permission from the rights-holder(s) directly, unless additional rights are indicated by a Creative Commons license in the record and/ or on the work itself. This Thesis has been accepted for inclusion in WVU Graduate Theses, Dissertations, and Problem Reports collection by an authorized administrator of The Research Repository @ WVU. For more information, please contact researchrepository@mail.wvu.edu. 

WAG EOR

Anthony William Morrow

Follow this and additional works at: https://researchrepository.wvu.edu/etd

Part of the Engineering Commons 


\title{
Confirmation of TDM Capabilities in Modeling Compartmentalized WAG EOR
}

Anthony William Morrow

Thesis submitted to Benjamin M. Statler college of Engineering and Mineral Resources at West Virginia University

\author{
In Partial fulfillment of the requirements for the degree of \\ Master of Science \\ In \\ Petroleum and Natural Gas Engineering \\ Shahab Mohaghegh, Ph.D. Chair \\ Mehrdad Zamirian, Ph.D. \\ Kashy Aminian, Ph.D. \\ Sam Ameri, M.S. \\ Department of Petroleum and Natural Gas Engineering \\ Morgantown, West Virginia \\ 2020
}

Keywords: Data-Driven Reservoir Modeling, Artifical Intrelligence and Data Mining, Top-Down-Model

Copyright 2020, Anthony Morrow 


\section{Abstract \\ Confirmation of TDM Capabilities in Modeling Compartmentalized WAG EOR}

\section{Anthony Morrow}

Data-Driven Reservoir Modeling (DDRM), commonly referred to as Top-Down Modeling (TDM), is a relatively new and cutting-edge approach to the traditional numerical reservoir modeling and simulation techniques. DDRM uses artificial intelligence and machine learning in tandem to construct full-field models using measured data instead of calculations that refer to equations derived from averaged values and type curves. TDM allows all of the measured data from a field to be combined and used towards generating predictions of the production on a well by well basis for a specific field.

Due to TDM not using the traditional physics-based approach, it is subjected to a plethora of criticisms within the industry. Therefore, the purpose of this thesis is to confirm the capabilities of TDM versus data synthetically generated using a Numerical Reservoir Simulator (NRS). To do this, the fluid flow through porous media will be modeled via the use of a traditional NRS; this way, everything is known about the reservoir in question. The data generated will then be exported and used towards the construction of the TDM.

To complete the proposed objectives of this thesis, an application will be used to aid in the development of a TDM. All of the data used in order to develop and history match the TDM will have been generated via the NRS; this is done to confirm the abilities of TDM forecasting existing wells behavior. Once the TDM has been constructed; the forecast data will be compared to that from the NRS to validate the ability of the TDM. 


\section{Dedication}

I would like to dedicate this thesis to the following individuals:

\section{My TFather}

For teaching me to not only persevere through the conflicts of life but face them head on, meanwhile remaining an honorable and truthful individual. I would not be the man that I am today without your teachings or continuous inspiration. I aspire to obtain a level of success comparable to your own.

\section{My Mother}

For telling me to believe in myself as I decided to alter the direction of my life in pursuance of my master's in engineering as well as providing a loving and caring home.

\section{Seredith @axner}

For being by my side throughout the struggles incurred during the completion of this thesis. Mitigating my obstinance with your continuous optimism, it is your love that aided me through one of the most difficult tests I have yet to face. I appreciate you in every way and am glad to have you as an integral piece of my life moving forward.

\section{Maxan Fayurood}

For inspiring me to return to school and continue to better myself through education, not to settle for something if it does not fulfil me in life. I wish you were still here to share this success with me. 


\section{Acknowledgement}

I would also like to thank Sam Ameri for the opportunities he has presented me with as well as all of the friendship that we have developed during my years with the university.

I would also like to acknowledge Dr. Shahab Mohaghegh for all that he has taught me. I hope to take all that he has instilled upon me and continue to contribute to the field of Oil and Gas. 
Table of Contents

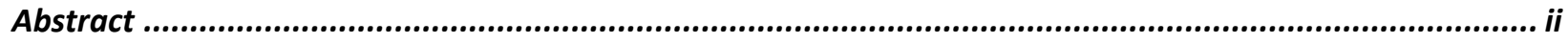

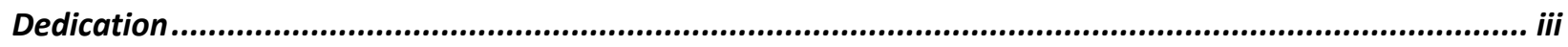

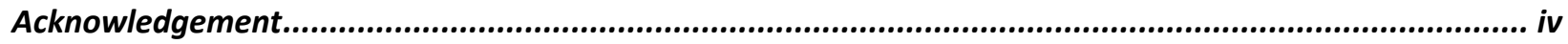

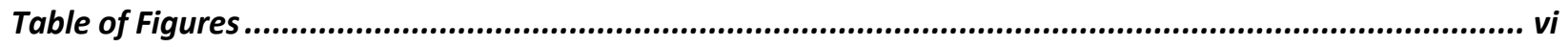

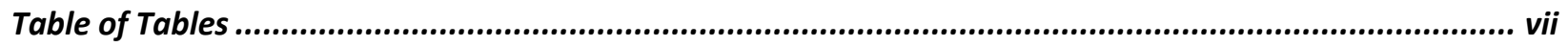

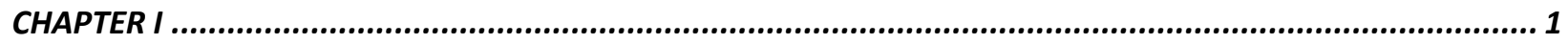

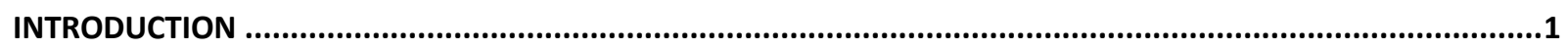

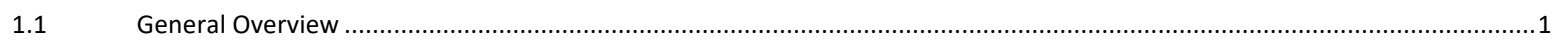

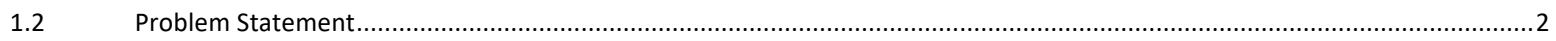

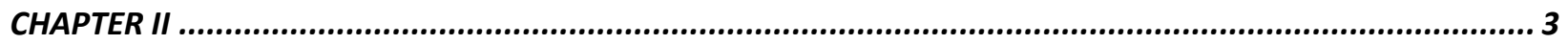

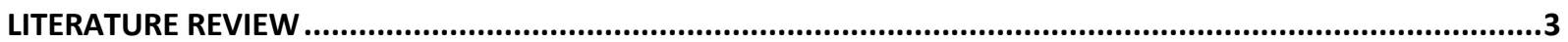

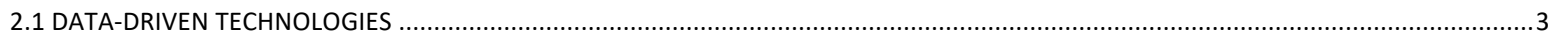

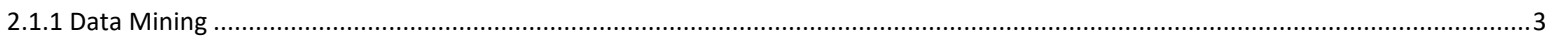

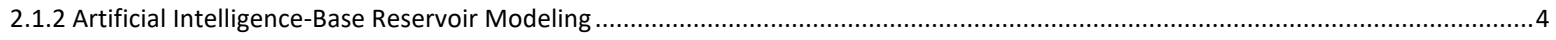

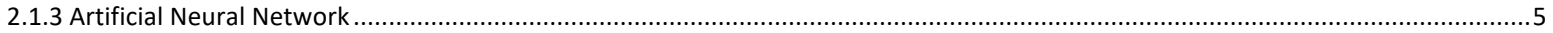

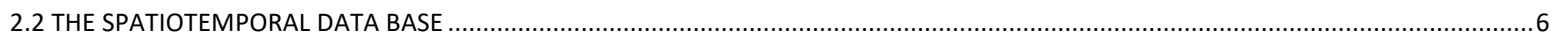

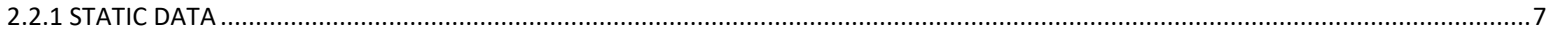

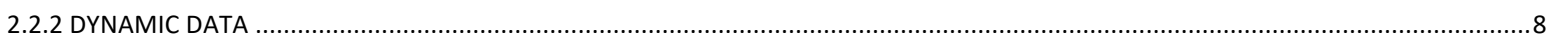

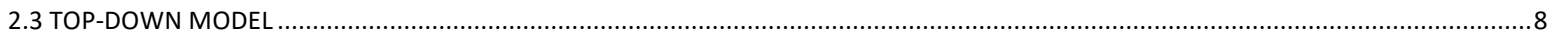

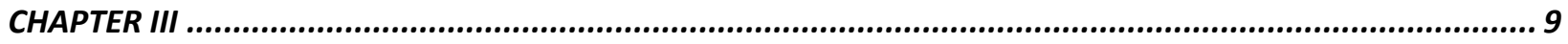

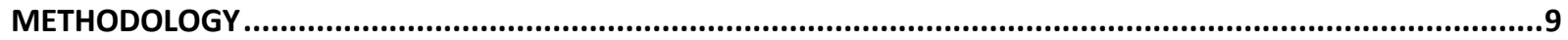

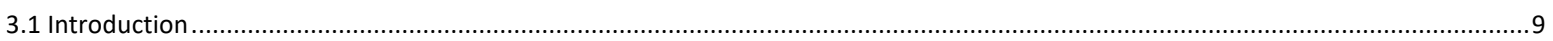

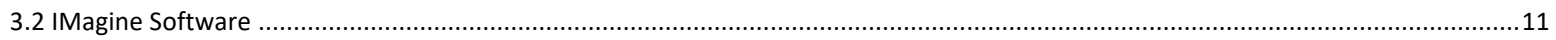

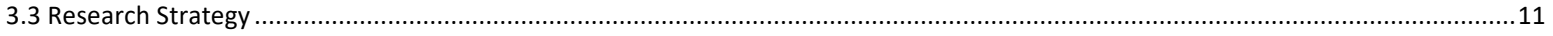

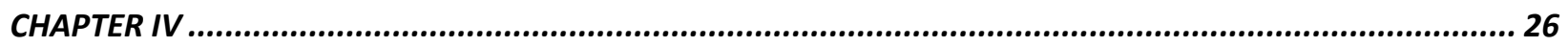

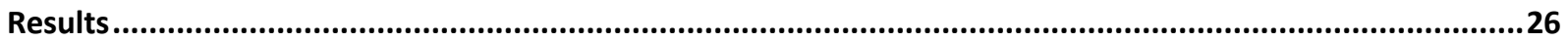

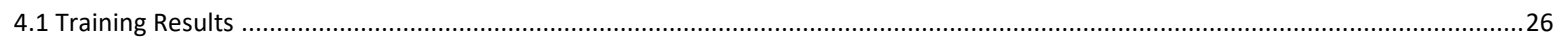

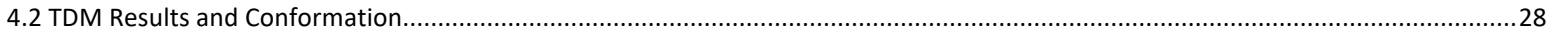

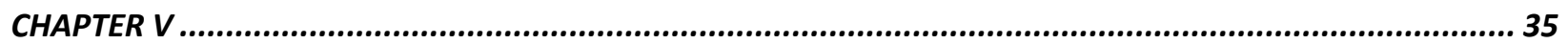

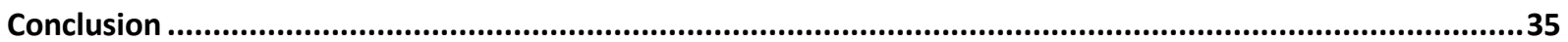

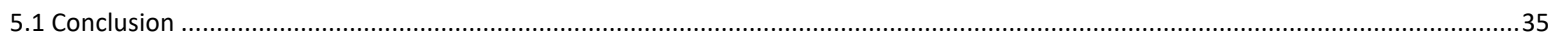

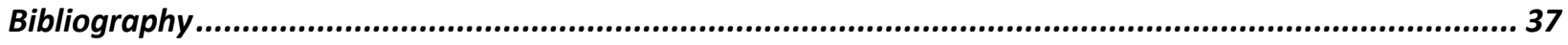

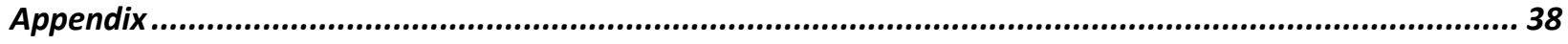

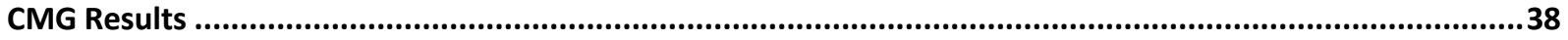

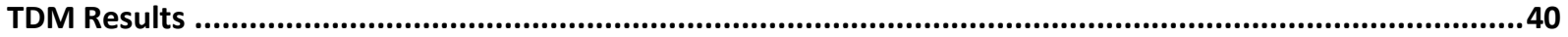

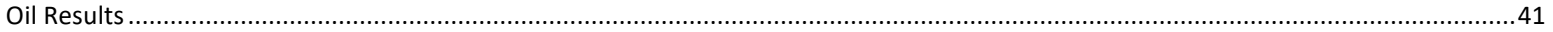

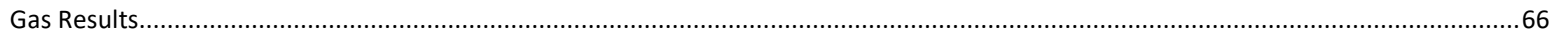

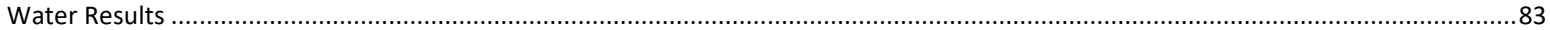

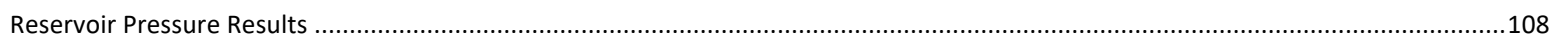

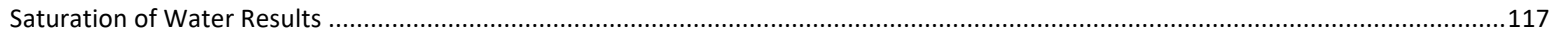




\section{Table of Figures}

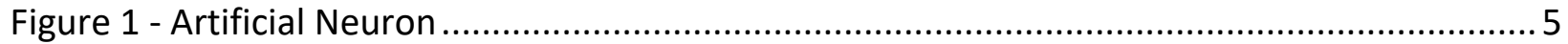

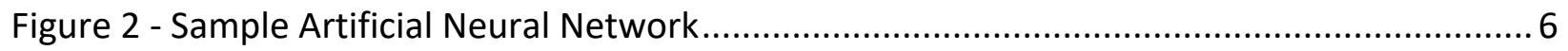

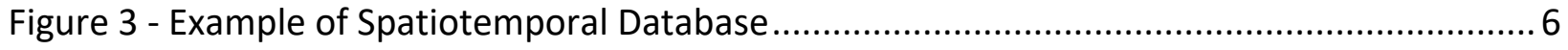

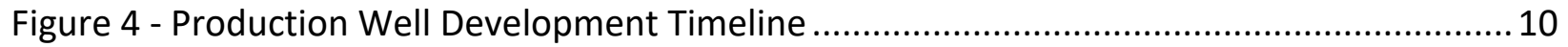

Figure 5 - Injection Development and Phase Timeline........................................................... 10

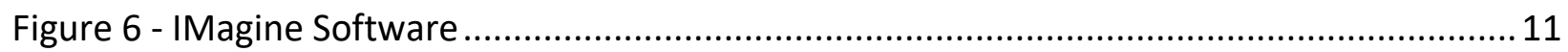

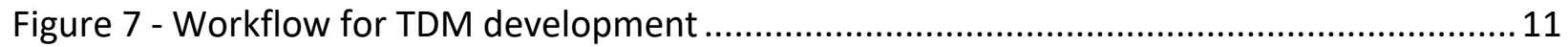

Figure 8 - Well Location as seen over Initial Water Saturation ................................................... 13

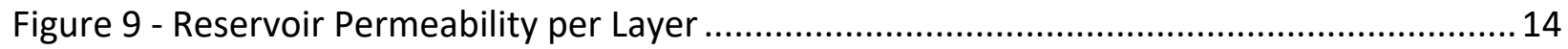

Figure 10 - Reservoir Permeability per Layer ..................................................................... 15

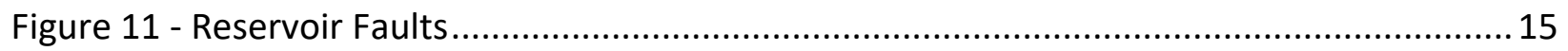

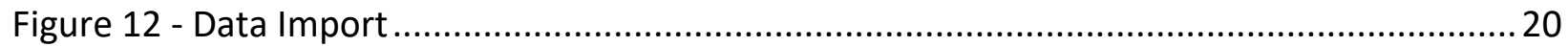

Figure 13 - Data Partitioning ............................................................................................... 21

Figure 14 - Reservoir Delineation Example ................................................................... 21

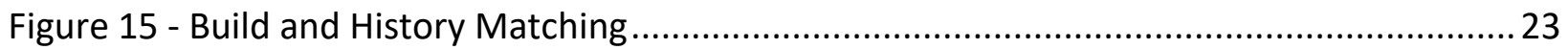

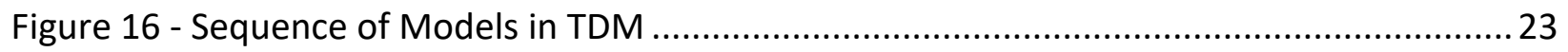

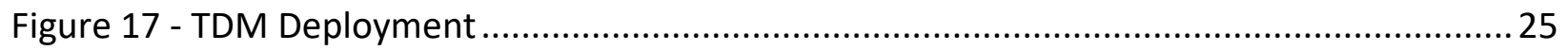

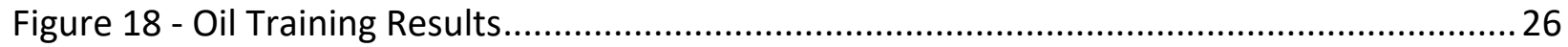

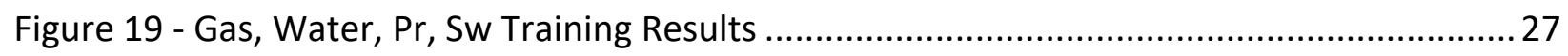

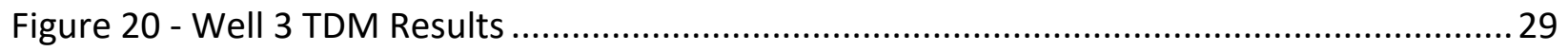

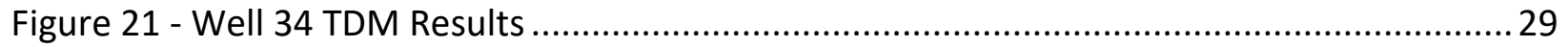

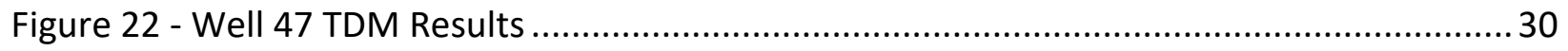

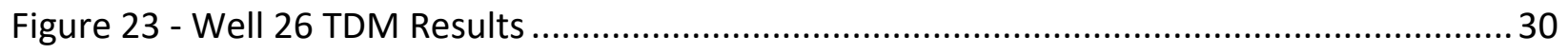

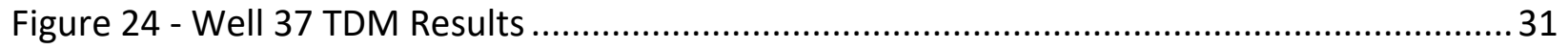

Figure 25 - Oil Production Entire Reservoir ..................................................................... 31

Figure 26 - Gas Production Entire Reservoir ................................................................ 32

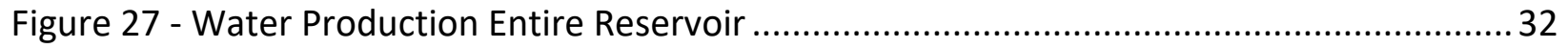

Figure 28 - Reservoir Pressure Forecasting Heat Map Summary ........................................... 33

Figure 29 - Water Saturation Forecasting Heat Map Summary .............................................34 


\section{Table of Tables}

Table 1 - Sample of Attributes used in Database ....................................................................

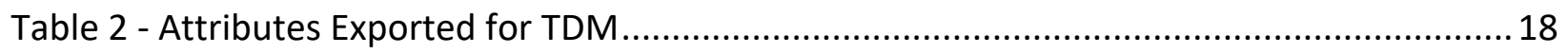

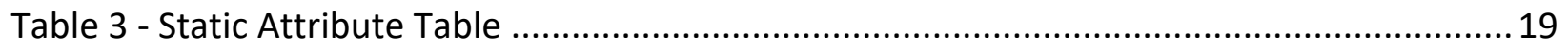

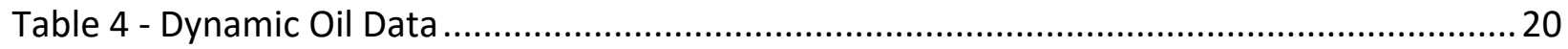

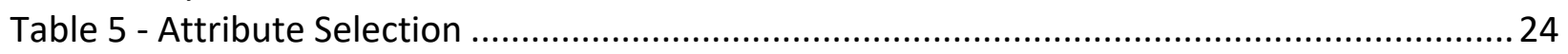

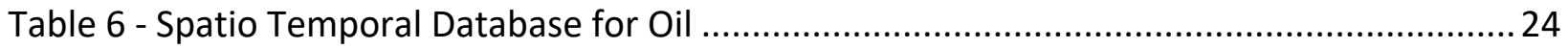

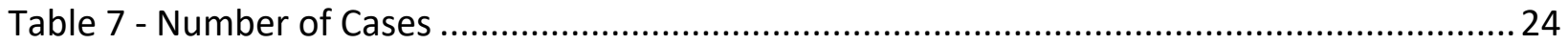

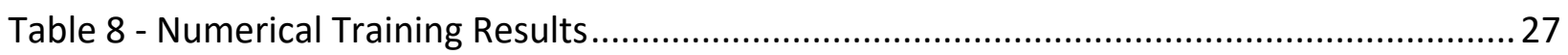




\section{CHAPTER I}

\section{INTRODUCTION}

\subsection{General Overview}

Reservoir modeling has been a standard in the oil and gas industry for years, being used and improved throughout the past century. Until relatively recently, Numerical Reservoir Simulation was the industry standard for modeling fluid flow through porous media. Within the past 20 years, there has been an emergence of the use of artificial intelligence and machine learning to model these same reservoirs and predict the fluid flow through porous media.

When using NRM, one of the significant assumptions that are made would be that all of the complexities of a reservoir are known and can be modeled. Supposing that the complexities are known, their modeling will be based on mathematical equations available to the modeler at the time of development. Along with this, one can conclude that since the reservoir model will be developed from the geological or static model, it will inherently carry assumptions and uncertain values. We, as engineers, however, feel comfortable changing these values to obtain an accurate history match based on gathered production data. Nevertheless, when it comes to developing these models in unconventional reservoirs, it is generally understood that these methods are far from the reality of the physics happening in shale reservoirs. They are only used due to the lack of any viable alternatives. (Mohaghegh, S. D., 2011, January).

Using artificial intelligence is highly successful due to its ability to use only field data when generating a model. These obtained data points are considered to be facts about the reservoir. By obtaining these facts about the reservoir, it eliminates any possibility for assumptions to be integrated into the data and alter the unseen patterns. The caveat to this form of data usage is the inherent noise that will be present due to the collection or real data, but this noise can be accounted for and mitigated. (Mohaghegh, Data-Driven Reservoir Modeling, 2017). 


\subsection{Problem Statement}

To adequately determine the characteristics and behavior of a reservoir, it is beneficial to have a model of the reservoir in question. Currently, the most generally accepted and commonly used method of modeling such reservoirs is by way of Numerical Reservoir Simulation. The problem with said technology is that it does not include a significant amount of the measured data once the field has matured and continues production.

When looking at a method such as decline curve analysis, operational constraints, and reservoir characteristics will not be brought into the decision-making process. Another element to keep in consideration is the amount of time invested in utilizing this approach once completed. Individuals managing reservoirs need to be able to make quick and accurate decisions with the tools provided to them. Data-Driven Reservoir Modeling (DDRM), also known as Top-Down Modeling (TDM), provides exactly that. It is an alternative to the traditional reservoir simulation models that have been created and utilized in the past. TDMs are comprehensive, full-field, empirical reservoir models that allow the measured data from the field to be brought into account when dealing with the reservoir. By using this approach, it will not modify the measured reservoir data that had been collected when history matching the model. Thus, history matching can be performed on multiple independent production values simultaneously. Due to this, for this case, synthetic data will be used to confirm the capabilities of TDM that were previously discussed.

The development of a DDRM is comprised of three phases: the first phase of this process, which is more exploratory, involves data mining. This is then followed by the development of the model based on the data obtained during phase one. This development consists of the training, history matching and validation of the model you are developing. The final phase of this process is then the post-model analysis. Using both data mining and artificial intelligence, due to the mining being more exploratory, it will involve the usage of unsupervised algorithms. When applying these steps in the case of reservoir engineering and modeling, modifications have been made to some of the traditional unsupervised algorithms to allow for the usage of reservoir engineering and geological domain expertise. (Mohaghegh, Data-Driven Reservoir Modeling, 2017). 


\section{CHAPTER II}

\section{LITERATURE REVIEW}

\subsection{DATA-DRIVEN TECHNOLOGIES}

In Mohaghegh's book "Data-Driven Reservoir Modeling," he defines Data-Driven Technologies as "a set of new technologies that rely on data rather than our current understanding of the physical phenomena in order to build models, solve problems, and make recommendations to help us in decisions" (Mohaghegh, Data-Driven Reservoir Modeling, 2017). When we apply this ideology to the concept of reservoir modeling, we can use measured data, also considered to be facts, to gain insight into the reservoir in question. Within these measured points, there will be inherent noise due to the differences in measurement, but this can be compensated for within the modeling approach.

\subsubsection{Data Mining}

Data mining is essentially the process of assessing the dataset and finding the inherent patterns that are present within it. By ascertaining the information about these patterns, they can be put to use in developing a deeper understanding of the structure in question. When this is applied to Reservoir Engineering, the comprehensive dataset is called a Spacio-Temporal Database, and this database is then used to determine the patterns within the data and eventually given to an algorithm for TDM. However, before the modeling can take place, the data has to be cleaned; this is done to remove any noise, missing data points or errors within the database. 


\subsubsection{Artificial Intelligence-Base Reservoir Modeling}

Artificial Intelligence (AI) falls into a subfield of computer science that is used explicitly to create machines that convey behaviors considered intelligent. However, AI can also be seen as an amalgamation of analytical tools in an attempt to imitate life. Researchers are continuously trying to develop and implement systems that can replicate or mimic things as complex as human thoughts. Some of the current uses of AI in the world are implemented through the usage of selfdriving cars, bank loan approvals, credit card fraud detection, medical diagnosis and a multitude of other processes that can be found throughout society. Although AI can be found so prominently throughout everyday functions, it is still broken into three main categories; these categories are fuzzy logic, artificial neural networks and evolutionary programming. Within the context of reservoir engineering tools developed from this technology are used to aid in understanding reservoir characterization, well log interpretations, drilling and pressure transient analysis (Mohaghegh, Shale Analytics, 2017).

In reservoir simulation and modeling, when applied to AI, the goal is to mimic patterns found within the data. Instead of using the conventional understanding of physics sheerly to model, it is used to aid the understanding of fluid flow through porous media. With this understanding, a spatiotemporal database can be constructed. This database is then used to train a predictive model. The training takes place via the modification of the connection between parameters. As training continuously, the algorithm will eventually converge to a state where it can mimic the behavior of the hydrocarbon reservoir, as seen within the provided data. Alternatively, instead of trying to use physics to show the trends within the data, we allow the algorithm to deduce the physics implicitly.

Although the concept of AI reservoir modeling is relatively new, it has an astonishing amount of potential. It allows reservoir models to be generated exponentially quicker compared to more traditional methods of modeling with this decrease in time to construct the model comes to a decrease in cost to develop reservoir models as well. 


\subsubsection{Artificial Neural Network}

As earlier stated, Artificial Neural Networks are considered to be one of the AI technologies that are present today. Systems like these are very complex such as the nervous system of the human body; they are continuously processing information to make decision-based on the data provided to it. Analyzing Figure 1, the decision process can be seen; the data from the previous neuron is provided to the one pictured. This input is affected by the weight of the connection determined during the training of the model, depicted as $\mathrm{W}_{1}, \mathrm{~W}_{2}$ and $\mathrm{W}_{\mathrm{a}}$, respectively. Once this weight has been applied, the data enters the neuron and is applied to the activation function, which dictates if the output will be sent (Shahkarami, A., Mohaghegh, S. D., Gholami, V., \& Haghighat, S. A., 2014, April).

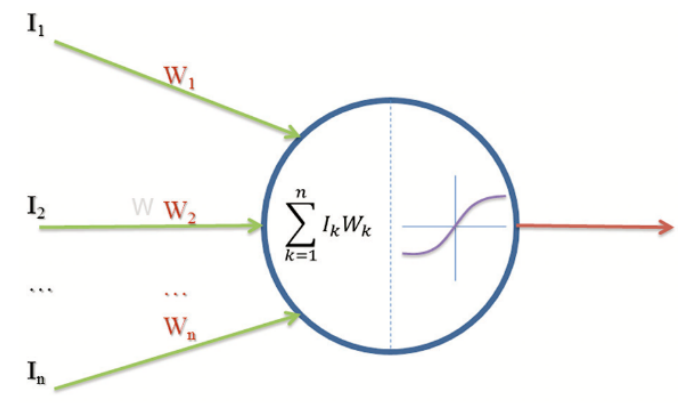

Figure 1 - Artificial Neuron

When discussing a neural network, it is a vast array of these neurons arranged in a particular order to execute specific functions. An example of such an order is called a multilayer network and can be seen in Figure 2. The first layer, called the input layer, will have the same number of neurons as attributes in the provided database. The second layer, also known as the hidden layer, the data is modified based on training. This modified data is determined based on the weights that it assigns to each connection. These hidden layers are often compared to a "Black Box," but unlike this accusation, they can be analyzed on a per-layer basis once the model is fully constructed. In a fully constructed network, there can be anywhere from no hidden layers to multiple, and it is dependent on the dataset and training performed. Lastly, as seen in the figure, the output layer is associated with the designated outputs of the model. In a fully constructed model, every output from a given layer is passed on to another node within the next layer. 


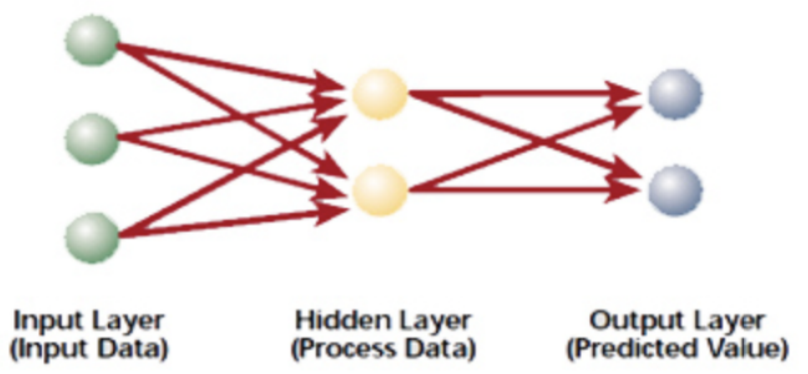

Figure 2 - Sample Artificial Neural Network

\subsection{THE SPATIOTEMPORAL DATA BASE}

The spatiotemporal database is what truly makes or breaks the process of TDM. This database provides all of the necessary field measurement material to the TDM in a simplistic format where the inherent trends within the dataset can be determined. To optimize the number of trends that the algorithms can find within the database the shape must be flat or a matrix consistent of only rows and columns. The rows of the given matrix correspond to a record from the dataset whereas the columns are the attributes associated for a given record. When dealing with these databases, there are two types of data types that they can provide: static data and dynamic data. An example of a generic spatiotemporal database can be seen below in Figure 3. The corresponding data to be used within a given data based can be found in Table 1.

\begin{tabular}{|c|c|c|c|c|c|c|c|c|c|c|}
\hline Well Name & Attribute 1 & Attribute 2 & Attribute 3 & Attribute 4 & Attribute 5 & Attribute 6 & Attribute 7 & Attribute 8 & Attribute 9 & Attribute 10 \\
\hline \multicolumn{11}{|l|}{ Well 1} \\
\hline \multicolumn{11}{|l|}{ Well 2} \\
\hline \multicolumn{11}{|l|}{ Well 3} \\
\hline \multicolumn{11}{|l|}{ Well 4} \\
\hline \multicolumn{11}{|l|}{ Well 5} \\
\hline \multicolumn{11}{|l|}{ Well 6} \\
\hline \multicolumn{11}{|l|}{ Well 7} \\
\hline \multicolumn{11}{|l|}{ Well 8} \\
\hline \multicolumn{11}{|l|}{ Well 9} \\
\hline Well 10 & & & & & & & & & & \\
\hline
\end{tabular}

Figure 3 - Example of Spatiotemporal Database 


\begin{tabular}{|c|c|c|}
\hline \multicolumn{3}{|c|}{ Static Attributes } \\
\hline Well Location & \multicolumn{2}{|c|}{ Formation Evaluation } \\
\hline $\begin{array}{c}\mathrm{X} \text { (Lattitude) } \\
\text { Y (Longitutde) } \\
\text { TVD }\end{array}$ & \multicolumn{2}{|c|}{$\begin{array}{c}\text { Formation Top } \\
\text { Initial Water Saturation } \\
\text { Pay Thickness } \\
\text { Permeability } \\
\text { Porosity }\end{array}$} \\
\hline \multicolumn{3}{|c|}{ Dynamic Attributes } \\
\hline Production & Stimulation & Well Tests \\
\hline $\begin{array}{c}\text { Oil } \\
\text { Gas } \\
\text { Water } \\
\text { Days of Production }\end{array}$ & $\begin{array}{c}\text { Water Injection } \\
\text { Gas Injection }\end{array}$ & Reservoir Pressure \\
\hline
\end{tabular}

Table 1 - Sample of Attributes used in Database

\subsubsection{STATIC DATA}

For an attribute to be classified as a static attribute in the development of the TDM it cannot change over the time. The data that falls within this classification is used to construct the geocellular model where the quantities of oil, gas and water present within the reservoir are calculated. This model is also known as the geological static model. When considering that all rocks can be classified as both heterogeneous and anisotropic, the static data can then be subdivided into two subcategories. The first category would be constructed of all of the attributes that are truly static such as the $X$ and $Y$ location of the well. This will not change for the life of the well at all. The second group would be dynamic-static data. This data contains dynamic attributes that are expected to change throughout the life of the reservoir being modeled. An example of this type of attribute would be drainage area, as new wells are completed within the reservoir the available drainage area to each well can alter over time. Along with this, some static attributes that are associated with drainage area such as the average porosity of the drainage area will also dynamically change over the life of the reservoir in question as well (Mohaghegh, Data-Driven Reservoir Modeling, 2017). 


\subsubsection{DYNAMIC DATA}

Dynamic data, as opposed to static, are attributes that will change over time. Most of the attributes that will fall within this class are due to human interventions within the production, regardless if said intervention was intentional or not. Things such as altering the size of the choke would fall within the domain of an intentional intervention. Whereas altering the well head pressure due to a constraint of the facilities downstream would be unintentional. It is important for the model to differentiate between the different types of data be it dynamic or static.

\subsection{TOP-DOWN MODEL}

Dr. Shahab Mohaghegh is seen as one of the early pioneers of developing Al technology in a manner to aid in petroleum engineering. Within the past few years, he has developed and introduced the world to the technology of TDM. This approach is considered by many to be the first approach to a data-driven reservoir model using comprehensive datasets based on actual data. TDM is such a critical technology within the industry because it simultaneously utilizes multiple disciplines. Some of these include reservoir engineering, statistical analysis using machine learning, reservoir modeling and a thorough understanding of AI. TDM was developed as an alternative to traditional modeling approaches in the sense that it is less demanding of both time and monitory resources to fully develop and complete a model of a reservoir. (Mohaghegh, S. D., Al-Mehairi, Y., Gaskari, R., Maysami, M., \& Khazaeni, Y., October, 2014).

Some of the features of TDM's that set them apart from traditional numerical approaches are the following points:

- It makes no assumptions or interpretations on the data that is given to it; meaning that the geological model that is provided on behalf of the modeling approach is unbiased.

- It does not attempt to make physics-based assumptions pertaining to fluid flow through porous media to model the production from the reservoir. 


\section{CHAPTER III}

\section{METHODOLOGY}

\subsection{Introduction}

To complete this project, IMagine software was used for the development of the TDM. The TDM was constructed based on synthetic data obtained from a numerical reservoir simulator. In this chapter, an in-depth description as to how the synthetic data was generated as well as it was implemented for the TDM will be covered. The numerical simulation will consist of 49 production wells and 20 injection wells. The injection wells will alternate between injecting water and gas injection to adequately model WAG recovery being implemented on this reservoir. Additionally, the reservoir in question has 6 layers but will not be producing from layers 3 and 5 . To fulfill this, all wells were not completed in either of these layers. Production from this reservoir started in January 1995 and continued until December of 2016. The production development plan consisted of 4 primary phases, and the final phase was broken into 4 sub phases. This phase development can be seen in Figure 4. The injection development phases and the WAG alteration plan can be seen in Figure 5 below. The reservoir being studied will have dome like features as well as a series of faults with no transmissibility across said fault to add more complexity and pressure boundaries present within the reservoir. 

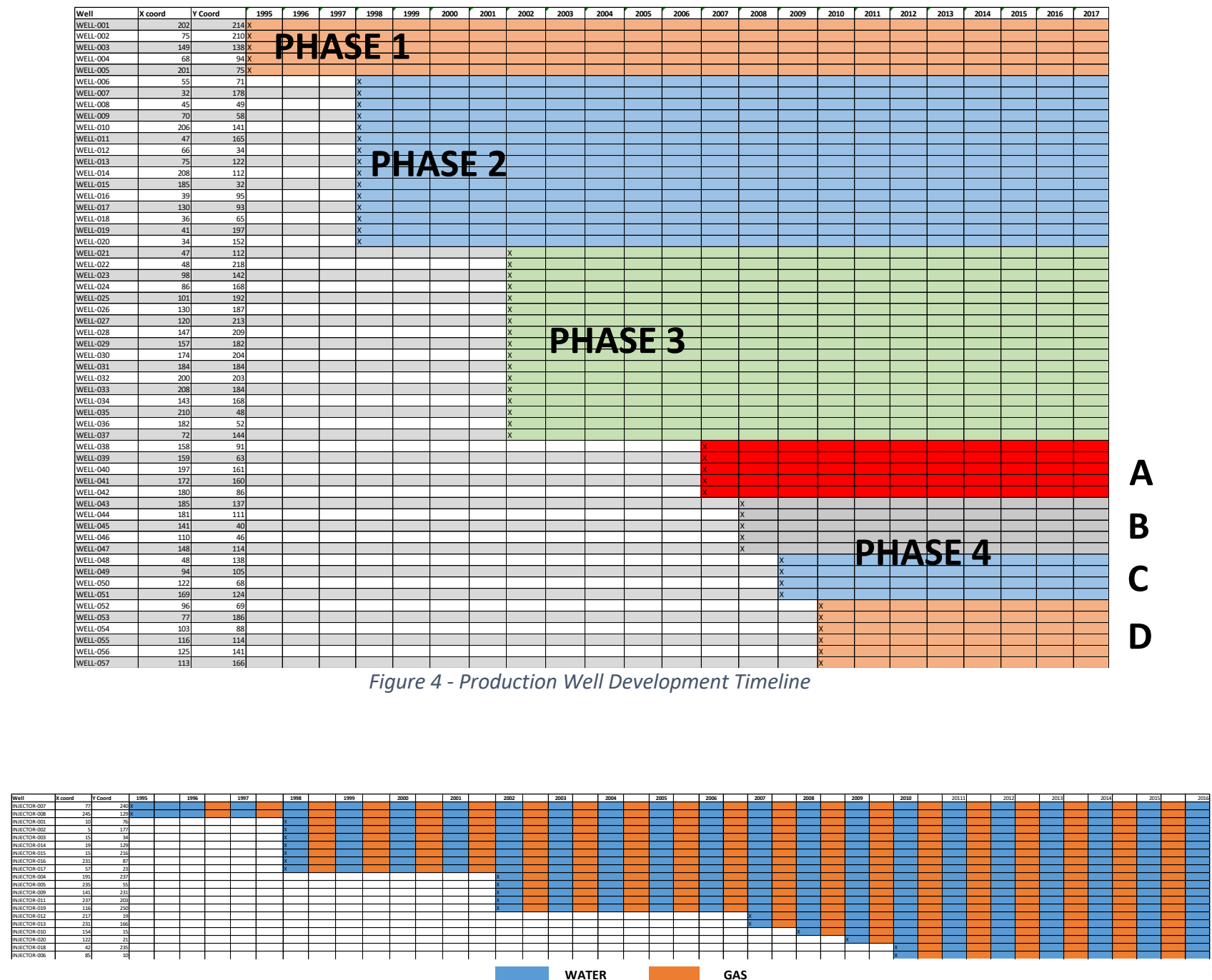

Figure 5 - Injection Development and Phase Timeline 


\subsection{IMagine Software}

The software used for the completion of this study, IMagine (Figure 6), is the only program of its kind. It allows for a thorough construction of TDM through a series of innovative algorithms. Based on artificial neural networks, fuzzy logic and genetic optimization, it aids in the development of comprehensive models.

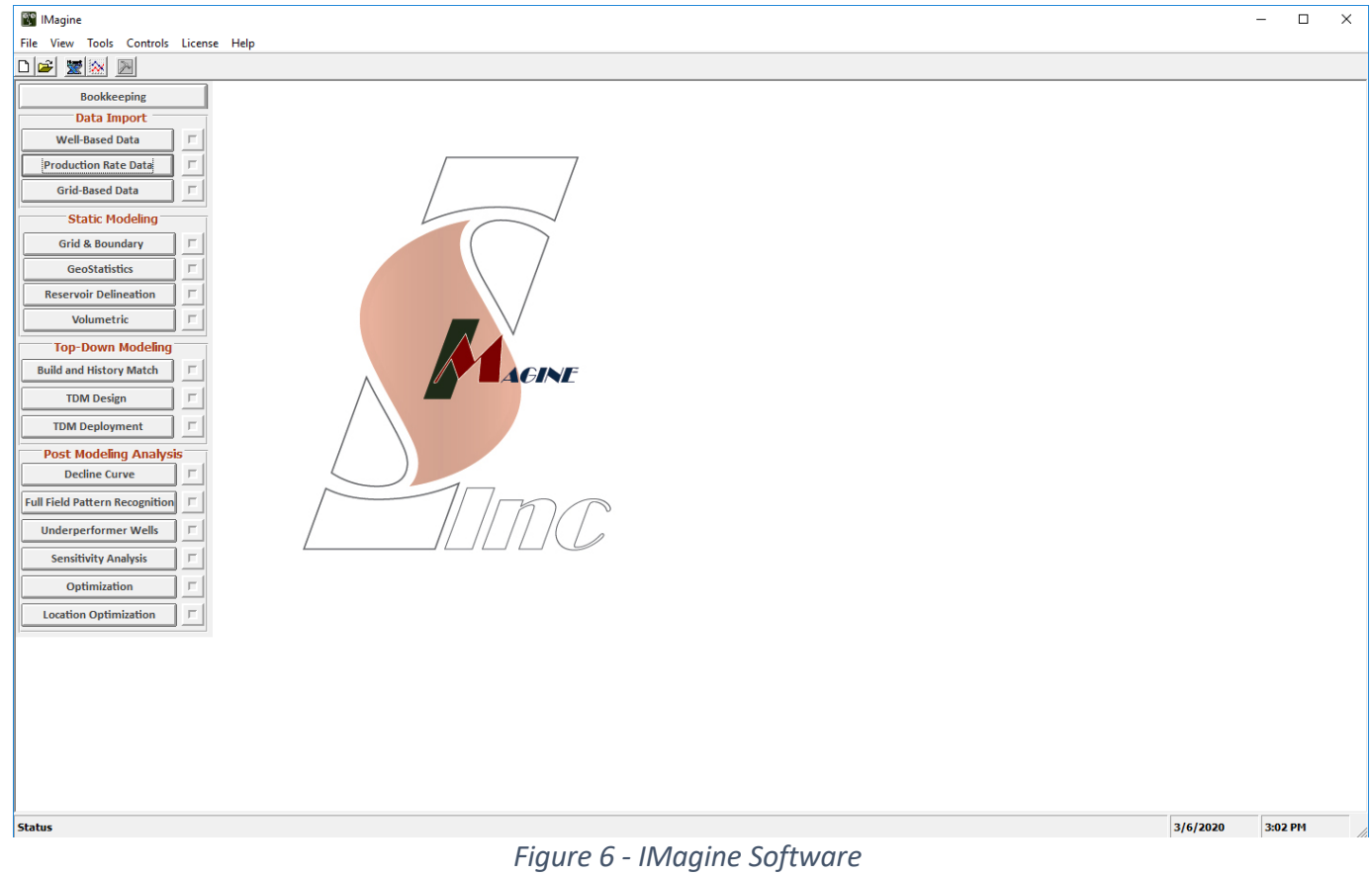

\subsection{Research Strategy}

Visualization of the workflow implemented throughout the completion of this study can be seen in the Figure 7 below.

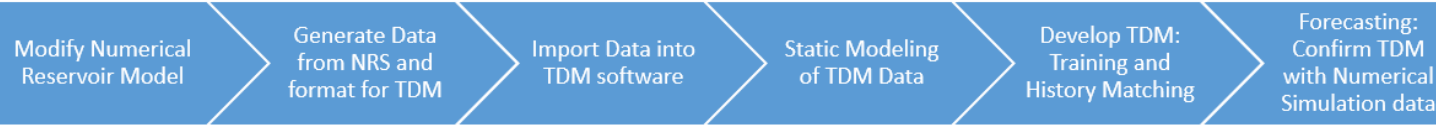

Figure 7 - Workflow for TDM development 


\section{Modify the Numerical Reservoir Simulator}

The model that was used for the purposes of this thesis was developed at WVU for studies such as this one to be conducted on. Once the model was obtained, a few tests were completed to verify that the model was in working order before modifications began. The overview of the well locations as well as the general shape of the reservoir can be seen below in Figure 8 and Figure 9. The permeability and porosity of each of the 6 layers can be seen in Figure 10 and Figure 11 respectively. Once these analyses had been complete, the model underwent alterations to fit the criteria of the thesis. Faults were added to the model to fulfil the required reservoir compartmentalization, these faults can be seen in Figure 12. All three of the faults had the transmissibility across them set to zero. This was done to create pressure boundaries within the reservoir and add complexity. Verification of the success of the faults instituting compartmentalization as well as pressure boundaries can be seen below in Figure 13, image shows the pressure distribution at a timestep within the reservoir's lifespan. Here it can clearly be seen that the boundaries are acting as they are expected to. Both layers 3 and 5 were closed to all production and injection wells to add more complexity to the reservoir. This stipulation was fulfilled by not completing any of the wells within either of the specified layers. The operational conditions were changed to have a daily injection volume for both water and gas injector wells. Determining the final rates to use was an iterative process due to the initial model being consistent of only water injection on the field in question. The volume of gas added had to compensate for the pressure loss due to the removal of water injection if there was not enough gas injected to compensate for the volume of water removed, then the differential pressure across the block over a time step would cause non-convergence errors within the simulation run. The final success of the WAG injection process can be seen below in Figure 14, here it is seen the saturations of water and gas change as the reservoir goes through the first phases of each fluid type. Along with this minimum oil rates as well as a water cut constraints was added to the model in order to prevent wells nearest to the injectors from eventually producing mostly all water and mitigating the effects of the injection procedure. A randomized shut-in program was used to make the model more realistic. As previously mentioned, there were 49 production wells as well as 20 injection wells. 


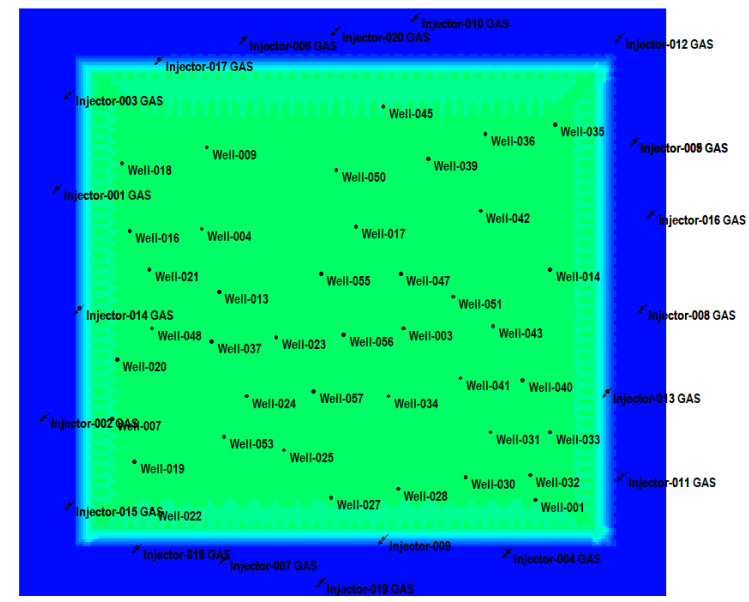

Figure 8 - Well Location as seen over Initial Water Saturation

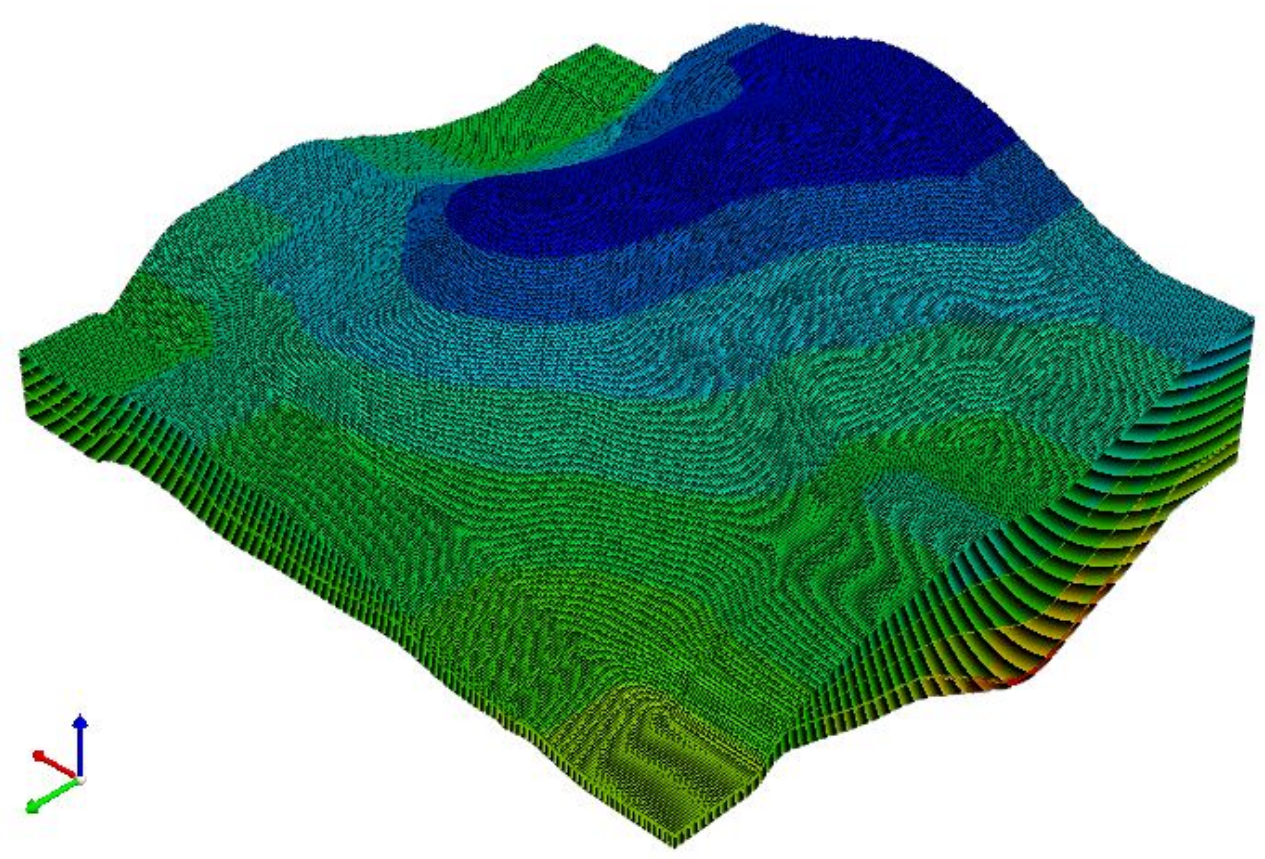

Figure 9-3D Visualization of Reservoir 

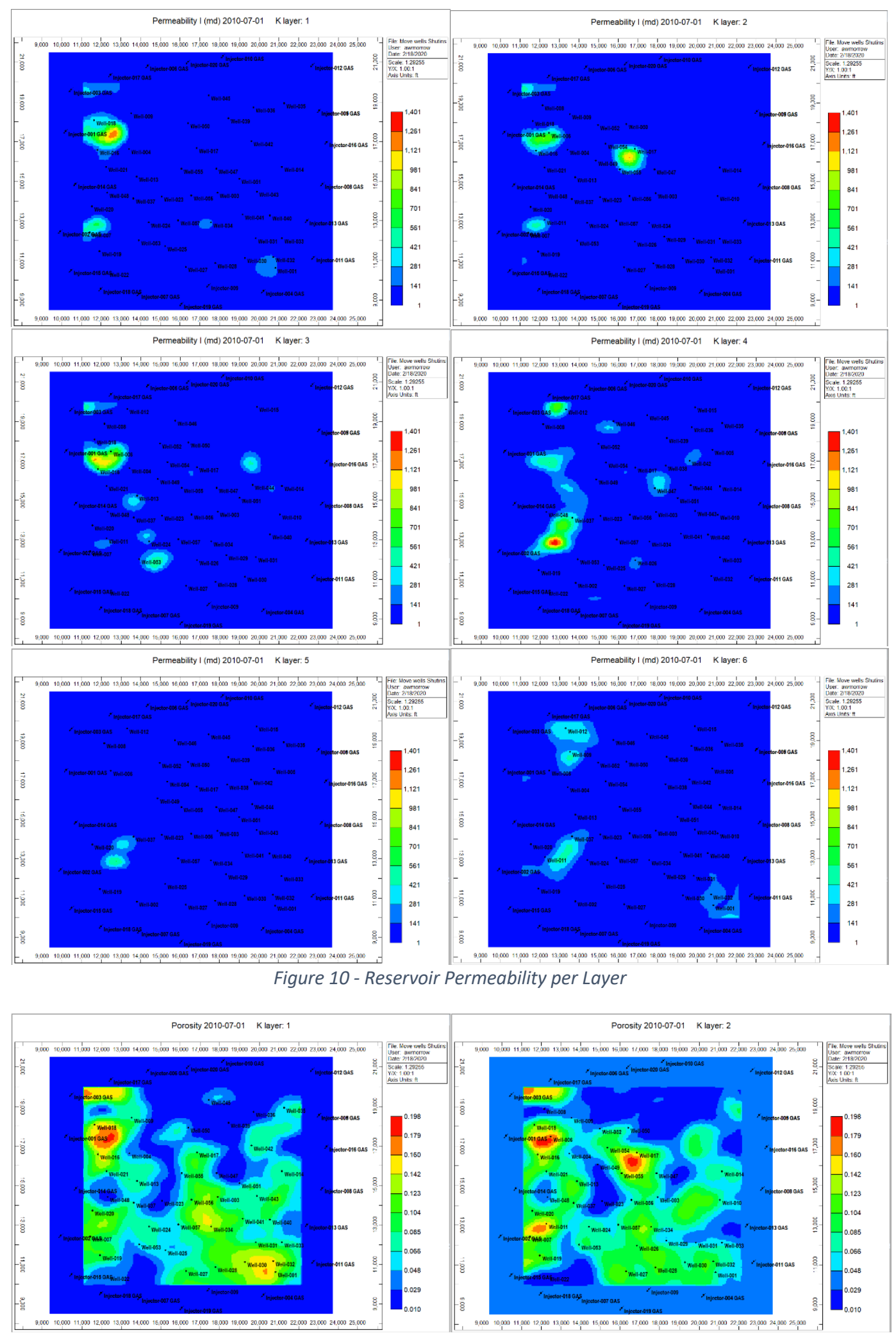


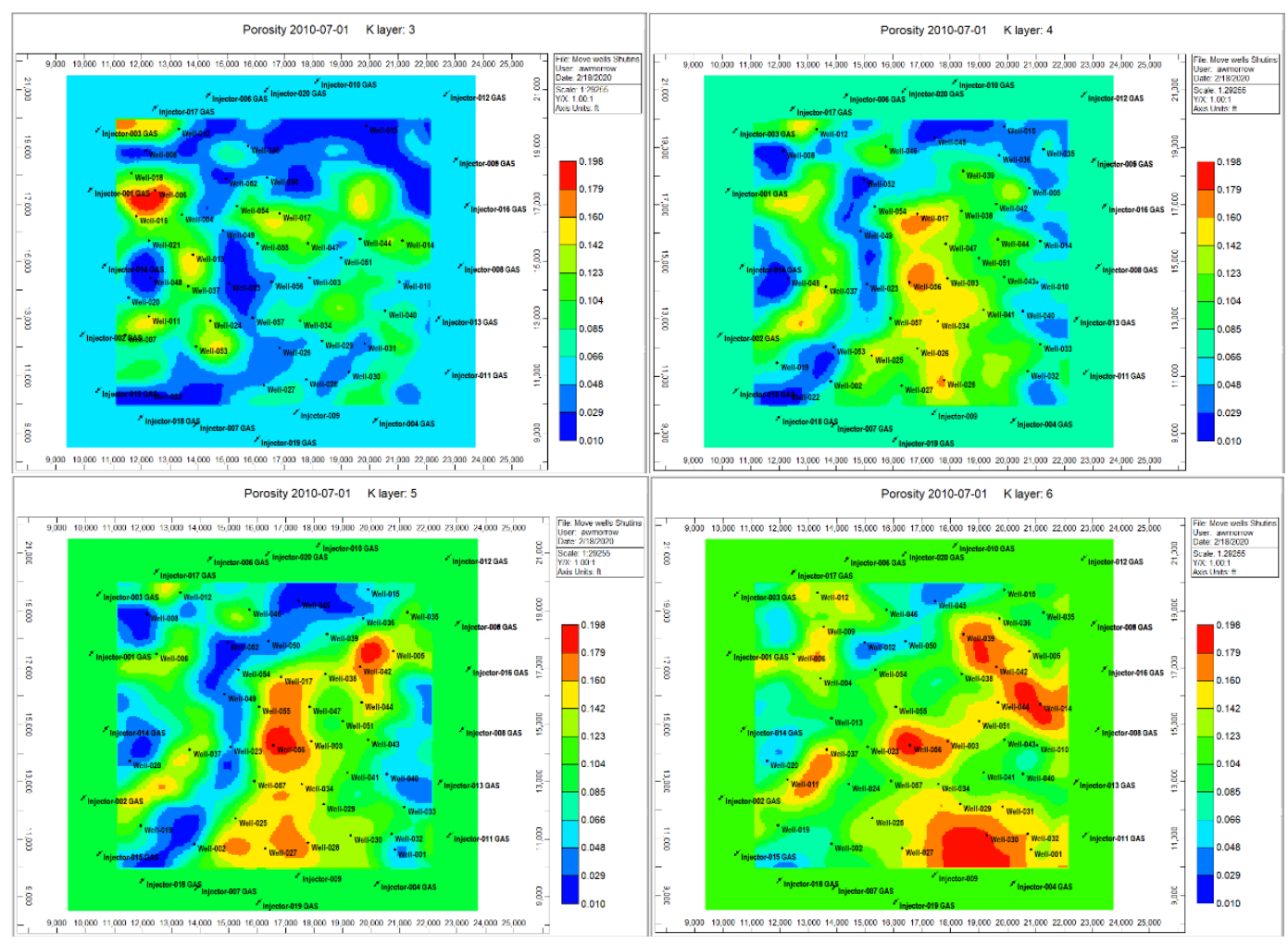

Figure 11 - Reservoir Permeability per Layer

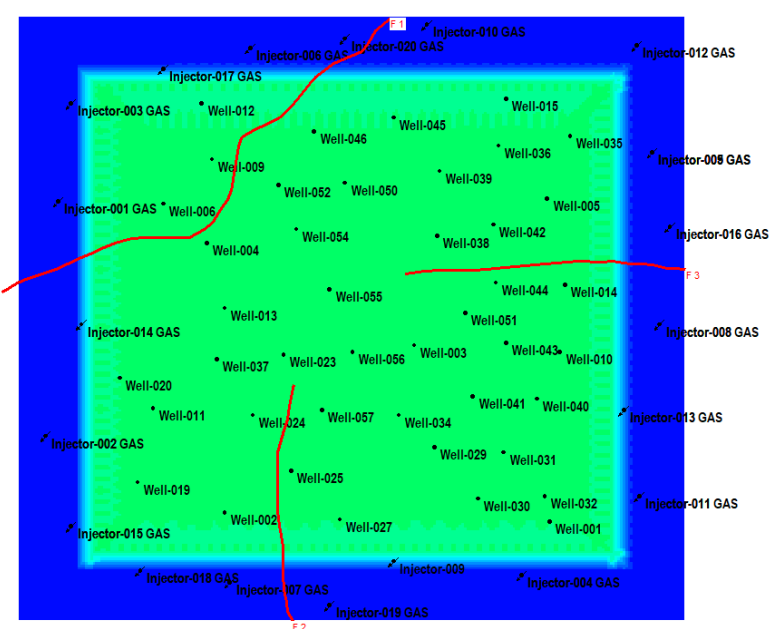

Figure 12 - Reservoir Faults 


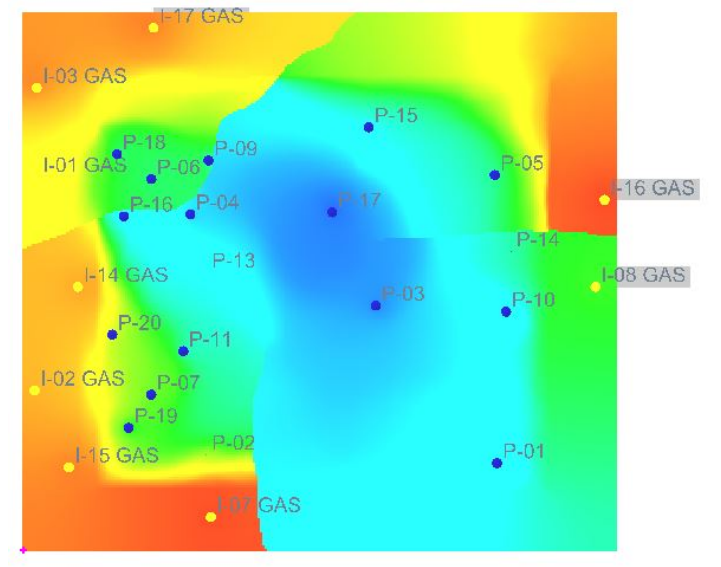

Figure 13 - Pressure Boundary

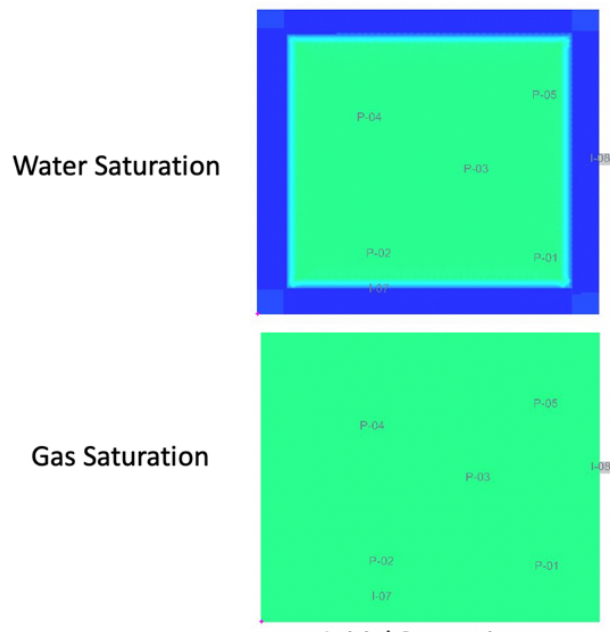

Initial Saturation
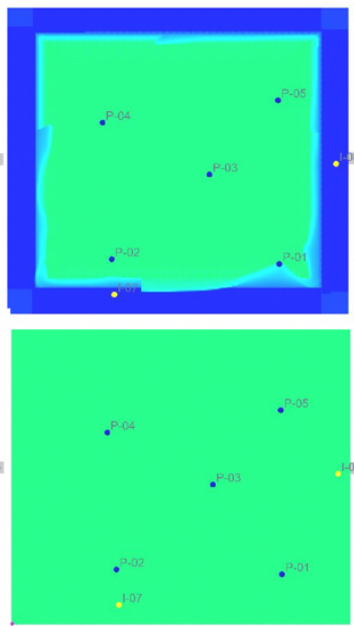

End Phase 1 Water Injection

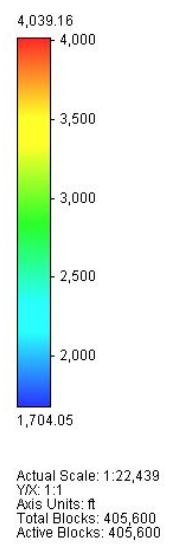

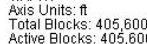
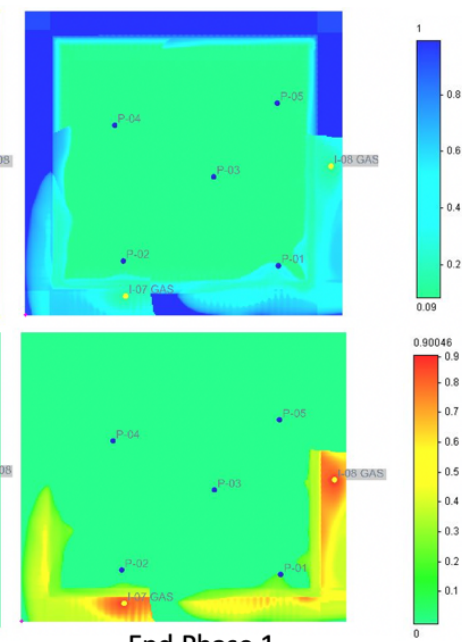

End Phase 1 Gas Injection

Figure 14 - Conformation of WAG Implementation

\section{Generate the required data for the Top-Down-Model}

Once the numerical model was completed and the results were deemed to be adequate for the purposes of this study the data needed to be exported and then converted to an acceptable format for the TDM program. From all of the data generated within the numerical simulation, the attributes as seen in Table 2 below were exported and given to the TDM software. The data was classified into two groups, static and dynamic data. These groups are separated on the basis of the value of that attribute will change throughout the window of simulation. If the attribute was deemed to fall within the static category, then the given information was exported on a per layer basis and then averaged or summed together dependent on the given attribute. The method of combining the data per layer can be seen under the method column in Table 2. Once this data 
was collected it needed to be formatted in a way for the software to adequately use it. The formatting used for the static dataset used within this study can be seen in Table 3. The dynamic attributes had to be formatted in such a way to train the model as to how they were changing per well over time. This information was exported on a cell level instead of per layer of the reservoir to more adequately calculate the needed values. Once these had been exported from CMG some of the attributes were weighted and others were summed across the corresponding blocks within a given well. This was done dependent on the attribute. The method applied to each attribute within the dynamic section can be found in Table 2. For attributes that were completed using weighted averaging, the weighted method was different per attribute. For example, flowing bottom hole pressure was weighted using only the thickness of the formation whereas reservoir pressure was weighted with both thickness and permeability. These averages were completed based off of the monthly exported block information. A piece of the final formatting for the dynamic oil data can been seen below in Table 4. Based on the oil production for the reservoir a days of production table was also completed. Due to there being a minimum oil production rate and water cut constraint implemented on the model, it can be said that when there is no oil production that the well is not producing at all. 


\begin{tabular}{|c|c|c|}
\hline & Data Name & Method \\
\hline \multirow{9}{*}{ 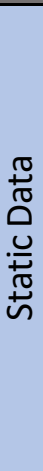 } & Well Type & $\mathrm{N} / \mathrm{A}$ \\
\hline & $x$ & $N / A$ \\
\hline & $Y$ & $N / A$ \\
\hline & Top & $\mathrm{N} / \mathrm{A}$ \\
\hline & TVD & $\mathrm{N} / \mathrm{A}$ \\
\hline & Initial Sw & Average \\
\hline & Pay Thickness & Sumation \\
\hline & Porosity & Average \\
\hline & Completion & Summation \\
\hline \multirow{11}{*}{ 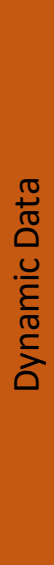 } & Oil Production & Summation \\
\hline & Gas Production & Summation \\
\hline & Water Production & Summation \\
\hline & FBHP & Weighted Average \\
\hline & Reservoir Pressure & Weighted Average \\
\hline & Oil Saturation & Weighted Average \\
\hline & Gas Saturation & Weighted Average \\
\hline & Water Saturation & Weighted Average \\
\hline & Days of Production & Summation \\
\hline & Water Injection Rates & Summation \\
\hline & Gas Injection Rates & Summation \\
\hline
\end{tabular}

Table 2 - Attributes Exported for TDM 


\begin{tabular}{|c|c|c|c|c|c|c|c|c|c|c|}
\hline Well Name & Well Type & $\mathrm{Xc}$ & Yc & Top & TVD & Swi & Thickness & Perm & Porosity & Completion Footage \\
\hline I-01 & Iv & 9724 & 17517.5 & 4358.253333 & 4404.5 & 1 & 44.745866667 & 100 & 0.1 & 44.745866667 \\
\hline $1-02$ & Iv & 9668.75 & 12362.35 & 4505.862222 & 4531.724444 & 1 & 23.84175778 & 100 & 0.1 & 23.84175778 \\
\hline $1-03$ & Iv & 9724 & 19669.65 & 4329.73 & 4364.478889 & 1 & 32.76715667 & 100 & 0.1 & 32.76715667 \\
\hline 1-04 & Iv & 20221.5 & 9459.45 & 4007.923333 & 4274.21 & 1 & 203.7323267 & 100 & 0.065 & 203.7323267 \\
\hline $1-05$ & IV & 23039.25 & 18568.55 & 4147.026667 & 4311.93 & 1 & 133.1482922 & 100 & 0.065 & 133.1482922 \\
\hline 1-06 & Iv & 14365 & 20820.8 & 4258.983333 & 4342.74 & 1 & 68.38636667 & 100 & 0.065 & 68.38636667 \\
\hline 1-07 & Iv & 13923 & 9309.3 & 3979.313333 & 4271.95 & 1 & 207.7200444 & 100 & 0.065 & 207.7200444 \\
\hline $1-08$ & IV & 23205 & 14864.85 & 4113.48 & 4287.64 & 1 & 137.7061289 & 100 & 0.065 & 137.7061289 \\
\hline 1-09 & Iv & 17459 & 9759.75 & 3911.526667 & 4260.32 & 0.551821333 & 235.8483667 & 100 & 0.065 & 235.8483667 \\
\hline $1-10$ & Iv & 18177.25 & 21321.3 & 4198.413333 & 4348.556667 & 1 & 115.9604467 & 100 & 0.065 & 115.9604467 \\
\hline I-11 & Iv & 22763 & 11161.15 & 4222.273333 & 4357.017778 & 1 & 99.23821111 & 100 & 0.065 & 99.23821111 \\
\hline $1-12$ & Iv & 22707.75 & 20870.85 & 4230.91 & 4370.97 & 0.934268 & 111.49413 & 100 & 0.065 & 111.49413 \\
\hline $1-13$ & Iv & 22431.5 & 13013 & 4186.175556 & 4343.852222 & 0.652117444 & 117.9717 & 100 & 0.065 & 117.9717 \\
\hline I-14 & Iv & 10718.5 & 14864.85 & 4176.87 & 4516.828889 & 0.652164444 & 233.6744922 & 100 & 0.065 & 233.6744922 \\
\hline 1-15 & Iv & 10497.5 & 10510.5 & 4121.888889 & 4444.943333 & 1 & 230.8298033 & 100 & 0.065 & 230.8298033 \\
\hline 1-16 & Iv & 23426 & 16966.95 & 4109.61 & 4265.08 & 1 & 122.0588556 & 100 & 0.065 & 122.0588556 \\
\hline $1-17$ & Iv & 12541.75 & 21121.1 & 4330.324444 & 4360.166667 & 1 & 28.04769111 & 100 & 0.1 & 28.04769111 \\
\hline 1-18 & Iv & 11989.25 & 9559.55 & 4079.475556 & 4362.401111 & 0.937699222 & 211.22611 & 100 & 0.065 & 211.22611 \\
\hline I-19 & Iv & 16077.75 & 8808.8 & 3899.906667 & 4259.68 & 1 & 237.4268 & 100 & 0.065 & 237.4268 \\
\hline $1-20$ & Iv & 16409.25 & 21021 & 4217.226667 & 4341.483333 & 1 & 95.08361667 & 100 & 0.065 & 95.08361667 \\
\hline$P-01$ & Pv & 20829.25 & 10610.6 & 4123.297778 & 4273 & 0.082184389 & 100.3025444 & 63.52325 & 0.08717557 & 100.3025444 \\
\hline $\mathrm{P}-02$ & $\mathrm{Pv}$ & 13812.5 & 10810.8 & 4023.616667 & 4302.295556 & 0.046370589 & 209.5878556 & 2.069399444 & 0.045334956 & 209.5878556 \\
\hline $\mathrm{P}-03$ & Pv & 17901 & 14414.4 & 3919.642222 & 4270.725556 & 0.037160111 & 243.5754111 & 20.26365972 & 0.118597456 & 243.5754111 \\
\hline P-04 & Pv & 13425.75 & 16616.6 & 4148.858889 & 4419.197778 & 0 & 197.7591978 & 38.48183639 & 0.082316231 & 197.7591978 \\
\hline P-05 & $\mathrm{Pv}$ & 20774 & 17567.55 & 4095.665556 & 4134.272222 & 0.046511667 & 38.605 & 1.956190556 & 0.066532544 & 38.605 \\
\hline P-06 & Pv & 12486.5 & 17467.45 & 4263.375556 & 4373.136667 & 0 & 55.63082222 & 376.6512778 & 0.143741611 & 55.63082222 \\
\hline P-07 & Pv & 12486.5 & 12262.25 & 4217.058889 & 4410.773333 & 0 & 98.72555556 & 92.8418 & 0.0945285 & 98.72555556 \\
\hline P-09 & Pv & 13867.75 & 17917.9 & 4197.375556 & 4295.034444 & 0 & 97.65927778 & 2.438473333 & 0.058949289 & 97.65927778 \\
\hline P-10 & Pv & 21050.25 & 14264.25 & 4098.468889 & 4305.946667 & 0.046374256 & 160.4357667 & 30.46674944 & 0.089310806 & 160.4357667 \\
\hline $\mathrm{P}-11$ & Pv & 13260 & 13313.3 & 4111.37 & 4415.735556 & 0 & 204.2574111 & 185.9187915 & 0.099720011 & 204.2574111 \\
\hline $\mathrm{P}-13$ & Pv & 13812.5 & 15215.2 & 4109.378889 & 4456.807778 & 0 & 244.8389989 & 76.36984444 & 0.100531222 & 244.8389989 \\
\hline$P-14$ & Pv & 21160.75 & 15715.7 & 4054.264444 & 4161.19 & 0.043490578 & 81.80547778 & 14.39334333 & 0.074308685 & 81.80547778 \\
\hline P-15 & $\mathrm{Pv}$ & 17735.25 & 18718.7 & 4113.022222 & 4163.427778 & 0.053320044 & 50.4056 & 1.216545556 & 0.01301875 & 50.4056 \\
\hline $\mathrm{P}-16$ & Pv & 11823.5 & 16566.55 & 4196.252222 & 4340.052222 & 0.081733722 & 143.7972667 & 243.1078889 & 0.130939778 & 143.7972667 \\
\hline P-17 & $\mathrm{Pv}$ & 16851.25 & 16666.65 & 4004.058889 & 4294.436667 & 0 & 207.6955556 & 135.6275272 & 0.119340897 & 207.6955556 \\
\hline P-18 & Pv & 11657.75 & 18068.05 & 4242.737778 & 4275.421111 & 0.092231478 & 32.68271111 & 369.8424444 & 0.136995444 & 32.68271111 \\
\hline P-19 & Pv & 11934 & 11461.45 & 4118.713333 & 4400.395556 & 0.075765856 & 189.0718444 & 31.03580741 & 0.0956155 & 189.0718444 \\
\hline$P-20$ & Pv & 11547.25 & 13713.7 & 4319.914444 & 4588.028889 & 0.094244011 & 140.6144711 & 4.361211481 & 0.060362622 & 140.6144711 \\
\hline P-21 & Pv & 12265.5 & 15715.7 & 4178.265556 & 4520.756667 & 0.009221573 & 238.1828544 & 30.095215 & 0.084188406 & 238.1828544 \\
\hline $\mathrm{P}-22$ & $\mathrm{Pv}$ & 12320.75 & 10410.4 & 4072.84 & 4358.233333 & 0.109436278 & 213.34573 & 1.458286944 & 0.033858256 & 213.34573 \\
\hline $\mathrm{P}-23$ & Pv & 15083.25 & 14214.2 & 4059.043333 & 4350.193333 & 0 & 218.3902522 & 5.2793025 & 0.047081278 & 218.3902522 \\
\hline $\mathrm{P}-24$ & Pv & 14420.25 & 12912.9 & 4076.184444 & 4383.453333 & 0.0414863 & 227.34607 & 11.52561222 & 0.079988064 & 227.34607 \\
\hline P-25 & Pv & 15249 & 11711.7 & 4149.874444 & 4313.014444 & 0.044487467 & 126.3260222 & 27.51719444 & 0.136403889 & 126.3260222 \\
\hline P-26 & Pv & 16851.25 & 11961.95 & 4033.668889 & 4275.218889 & 0.046356889 & 166.9091222 & 105.4412389 & 0.144704778 & 166.9091222 \\
\hline$P-27$ & Pv & 16298.75 & 10660.65 & 3901.105556 & 4092.874444 & $\begin{array}{l}0.050819 \\
\end{array}$ & 157.9334889 & 35.75531704 & 0.098638656 & 157.9334889 \\
\hline P-28 & $\mathrm{Pv}$ & 17790.5 & 10860.85 & 3945.755556 & 4127.037778 & 0.045220433 & 137.9545667 & 26.3513037 & 0.121875544 & 137.9545667 \\
\hline P-29 & Pv & 18343 & 12212.2 & 4108.862222 & 4273.797778 & 0.041996722 & 125.9678556 & 20.16001667 & 0.137773167 & 125.9678556 \\
\hline$P-30$ & $\mathrm{Pv}$ & 19282.25 & 11111.1 & 4007.961111 & 4290.333333 & 0.047057222 & 215.8863633 & 47.14674722 & 0.132264011 & 215.8863633 \\
\hline$P-31$ & $\mathrm{PV}$ & 19834.75 & 12112.1 & 4052.348889 & 4303.124444 & 0.043602344 & 192.4337644 & 27.48996806 & 0.096436956 & 192.4337644 \\
\hline P-32 & Pv & 20718.75 & 11161.15 & 4117.811111 & 4225.007778 & 0.046334767 & 107.1946556 & 78.91058111 & 0.121886589 & 107.1946556 \\
\hline$P-33$ & Pv & 21160.75 & 12112.1 & 4153.061111 & 4242.914444 & 0.043510467 & 89.85442222 & 3.266722778 & 0.066622683 & 89.85442222 \\
\hline$P-34$ & Pv & 17569.5 & 12912.9 & 3919.832222 & 4269.655556 & 0.0415137 & 242.2014667 & 62.38331944 & 0.133257417 & 242.2014667 \\
\hline$P-37$ & $\mathrm{Pv}$ & 13646.75 & 14114.1 & 4120.255556 & 4480.573333 & 0 & 151.0529689 & 215.47618 & 0.118531548 & 151.0529689 \\
\hline P-38 & $\mathrm{PV}$ & 18398.25 & 16766.75 & 4007.662222 & 4277.286667 & 0 & 176.6166 & 17.49285074 & 0.096772122 & 176.6166 \\
\hline P-39 & Pv & 18453.5 & 18168.15 & 4084.144444 & 4258.995556 & 0 & 127.2268333 & 23.25029296 & 0.094666733 & 127.2268333 \\
\hline P-41 & Pv & 19171.75 & 13313.3 & 4172.91 & 4282.202222 & 0.038999689 & 95.95498889 & 23.49308444 & 0.120623056 & 95.95498889 \\
\hline$P-42$ & $\mathrm{Pv}$ & 19613.75 & 17017 & 4149.635556 & 4267.655556 & 0.042333144 & 99.39841111 & 120.3275556 & 0.133382483 & 99.39841111 \\
\hline P-43 & Pv & 19890 & 14464.45 & 4038.691111 & 4295.008889 & 0.040652333 & 196.3503111 & 16.27456222 & 0.1007342 & 196.3503111 \\
\hline$P-44$ & $\mathrm{Pv}$ & 19669 & 15765.75 & 3984.922222 & 4277.521111 & 0.042333367 & 211.3189333 & 13.97312583 & 0.085518628 & 211.3189333 \\
\hline$P-47$ & Pv & 17845.75 & 15615.6 & 3913.453333 & 4278.015556 & 0.031641367 & 250.5424333 & 76.20079278 & 0.089283694 & 250.5424333 \\
\hline P-49 & Pv & 14862.25 & 16066.05 & 4089.591111 & 4374.516667 & 0 & 211.8414433 & 6.034539167 & 0.054675431 & 211.8414433 \\
\hline P-50 & Pv & 16409.25 & 17917.9 & 4113.377778 & 4313.983333 & 0 & 155.2309956 & 3.476153889 & 0.054495428 & 155.2309956 \\
\hline$P-51$ & Pv & 19006 & 15115.1 & 3952.136667 & 4274.024444 & 0.037952122 & 228.7757222 & 7.635279444 & 0.078738911 & 228.7757222 \\
\hline P-52 & Pv & 14972.75 & 17867.85 & 4166.59 & 4398.866667 & 0 & 168.9607067 & 2.169341944 & 0.037089247 & 168.9607067 \\
\hline P-53 & Pv & 13923 & 12012 & 4077.141111 & 4379.94 & 0.043001122 & 224.8286922 & 13.64446722 & 0.068782044 & 224.8286922 \\
\hline P-54 & Pv & 15359.5 & 16916.9 & 4102.676667 & 4370.477778 & 0 & 198.4797789 & 23.31033528 & 0.071827231 & 198.4797789 \\
\hline P-55 & Pv & 16077.75 & 15615.6 & 4009.043333 & 4297.305556 & 0 & 210.7863778 & 63.26158222 & 0.102820736 & 210.7863778 \\
\hline P-56 & $\mathrm{Pv}$ & 16575 & 14264.25 & 3937.353333 & 4288.597778 & 0.036666667 & 247.7304444 & 33.61684917 & 0.128098175 & 247.7304444 \\
\hline$P-57$ & Pv & 15912 & 13013 & 3983.35 & 4291.483333 & 0.0415137 & 224.8585889 & 6.824005833 & 0.083751336 & 224.8585889 \\
\hline
\end{tabular}

Table 3 - Static Attribute Table 


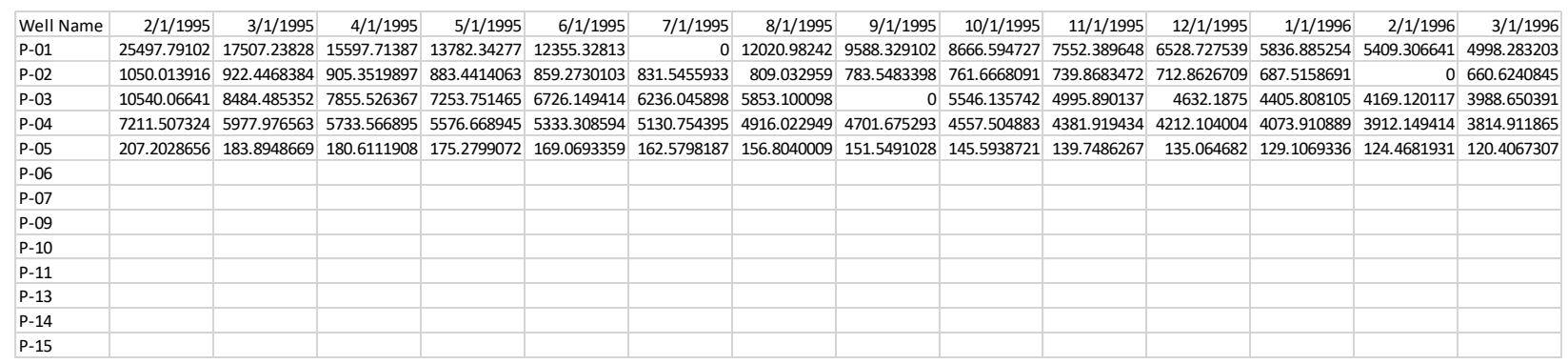

Table 4 - Dynamic Oil Data

\section{Importing Data into Imagine}

Once all of the data had been converted into the appropriate formats for IMagine, it then needed to be imported. All of the generated data was broken into two groups, either well based data or production data. They were separated like this to fulfill the criteria set by IMagine. Once selected, they were imported into their respective categories as seen in Figure 15. For training purposes and to validate the ability of the TDM to adequately forecast production from the reservoir data from 1995 until 2014 was given to the software. The partitioning of said data can be seen in Figure 16 below.

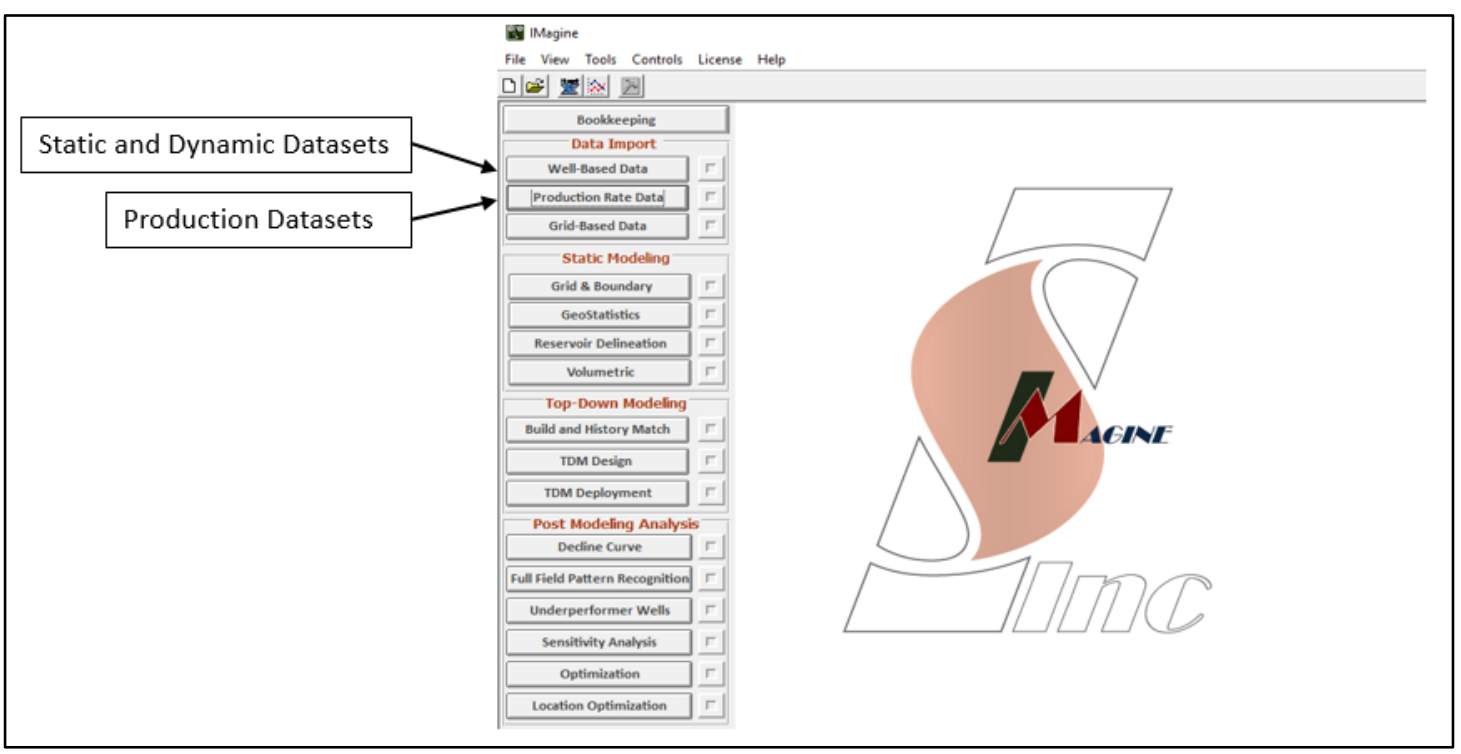

Figure 15 - Data Import 


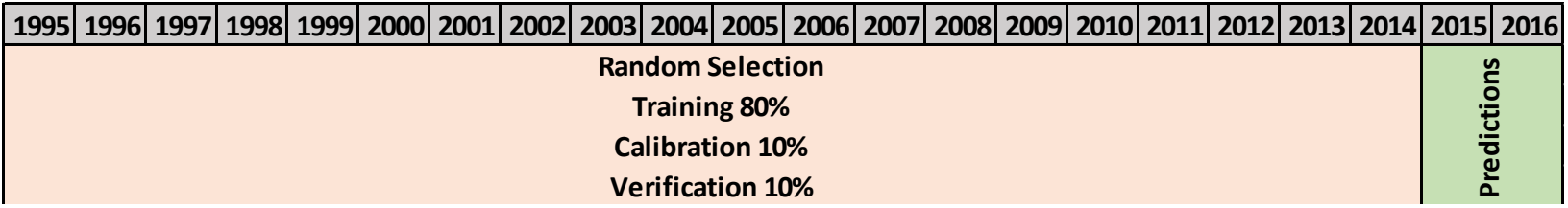

Figure 16 - Data Partitioning

\section{Static Modeling}

This section of the application allows for the completion of a grid and boundary analysis as well as geo-statistics, reservoir delineation and volumetric calculations to be performed. All of these calculations are based on the given factual data for the reservoir in question. The reservoir boundary had to be set manually for the model. Once the boundary was developed, a grid size was determined and then the reservoirs delineation was calculated using the Voronoi technique. When completed, the volumetric calculations could be completed. An example of the completed calculations for reservoir delineation can be found below in Figure 17.

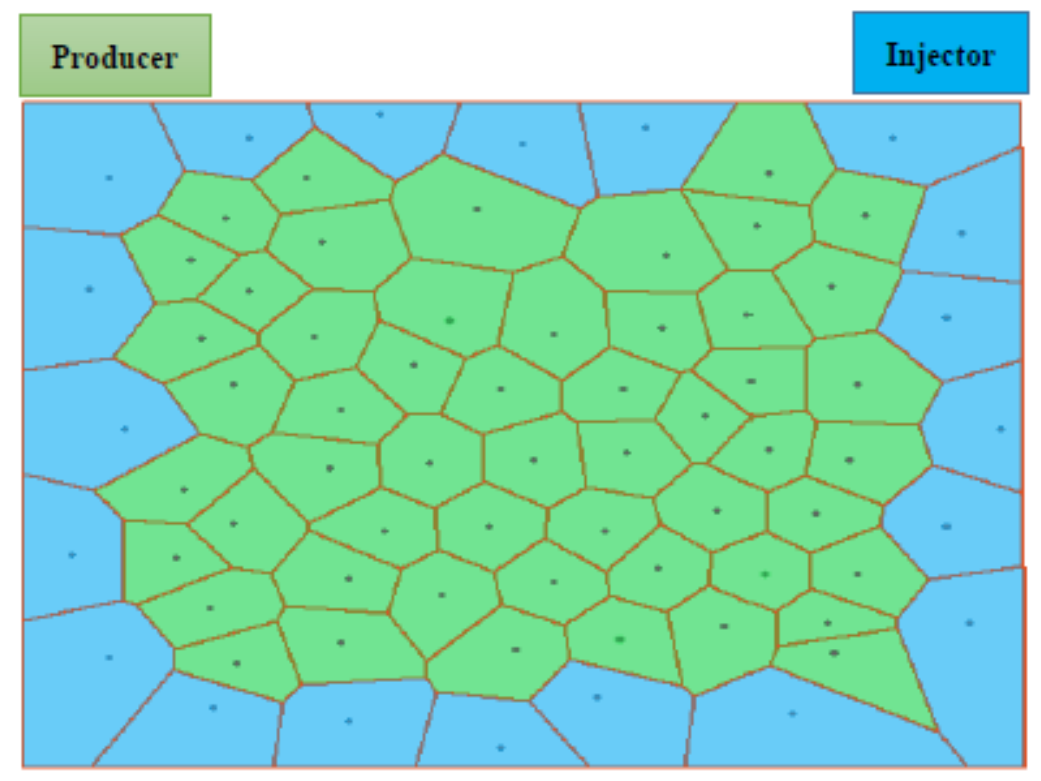

Figure 17 - Reservoir Delineation Example 


\section{Top-Down modeling: (Training and History Matching)}

This section is comprised of three steps; model design, attribute selection and development of a comprehensive dataset. These three steps can be seen highlighted in Figure 18 below. The first step to building each model is to determine out what the model's inputs and outputs will be. This will include all of the data that will be used to train the model within the date range specified. Once the data desired has been decided upon, the necessary attributes that each model will use during the training phase need to be selected. These attributes that are selected in this phase will go on to build the spatiotemporal database that is used for each model's data set. Each of the models used to construct this TDM used different attributes. The attributes that each model used was dependent on their place within the final TDM. The order of the models in the final TDM can be seen in Figure 19. For each model, the static data along with the dynamic data, comprised of operational conditions needed to be selected at time $(t)$. However, the productionbased models, for example, Oil at time (t-1) had to be selected and used due to Oil at time ( $t$ ) being the output of that model. Similarly, for Gas and Water their respective attribute had to be selected at time ( $t-1)$, however, the prior levels of the TDM was given to the current model at time ( $t$ ). For example, when building the water model we know the water( $t$ ) will be our output for the model. In terms of inputs, we will need to give it water(t-1) and due to its position in the TDM it will also receive oil( $(t)$ and gas $(t)$. A visual representation of the attribute selection can be seen in Table 5. 


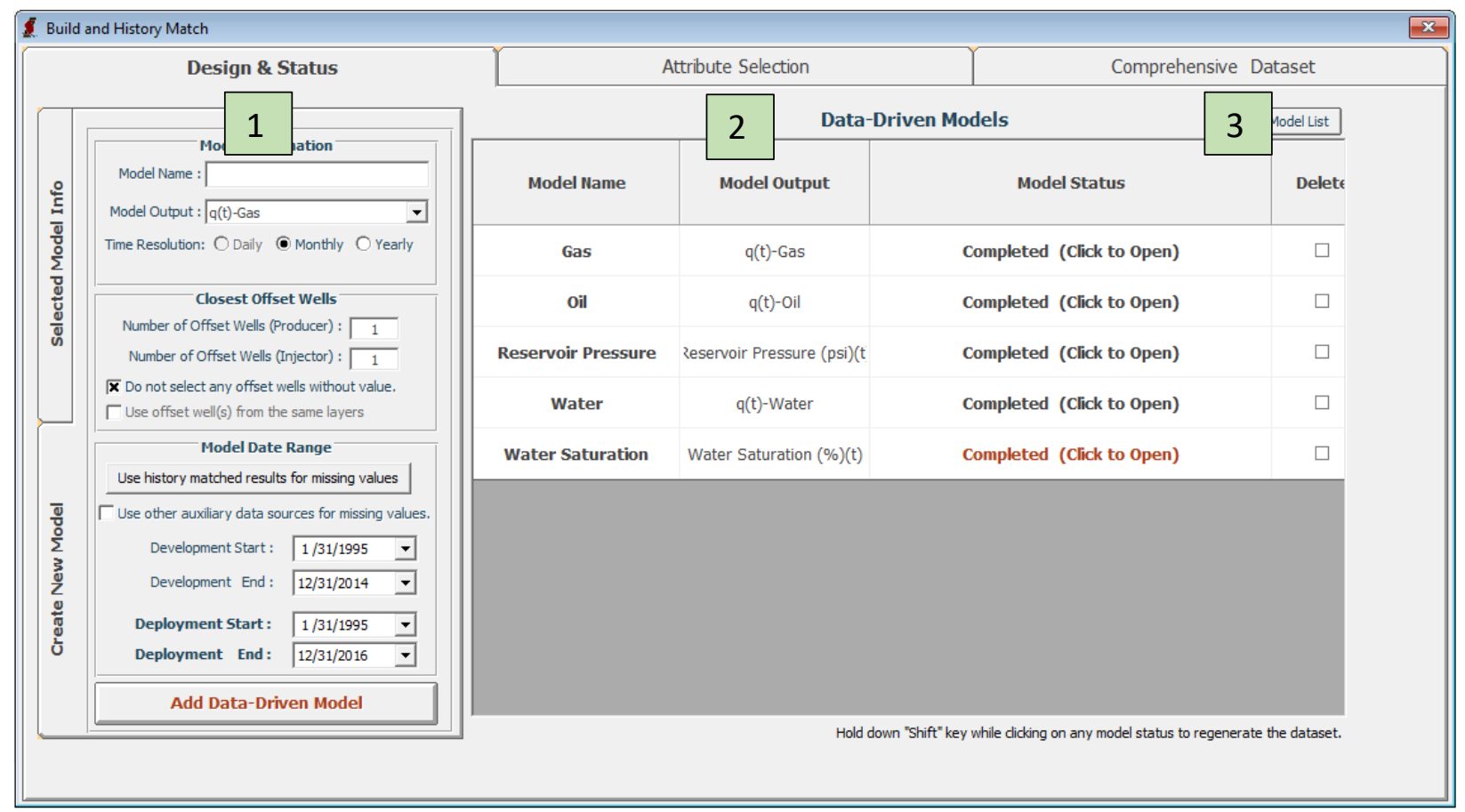

Figure 18 - Build and History Matching

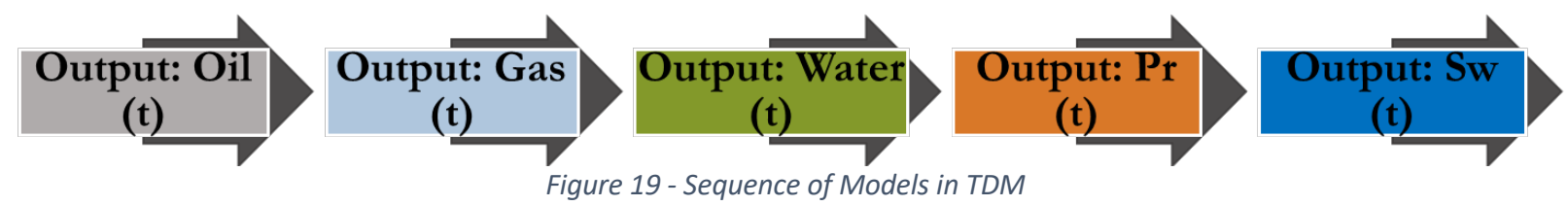

One important consistency among all of the models attribute selection for creating the spatiotemporal database was that oil(t) was selected before generating the dataset. This was due to the fact that the dataset was cleaned based on the oil production. Once the dataset was generated the values for oil were sorted numerically and all records where oil(t) equaled zero were removed from the dataset before advancing to the training section of model development. This is due to wanting the model to be trained on data points where there was oil production since that was the most important element of the TDM. A cumulative list of the attributes that were selected for each model can be seen below in table below in Figure 19. A generated and cleaned database can be seen below in Table 6. 


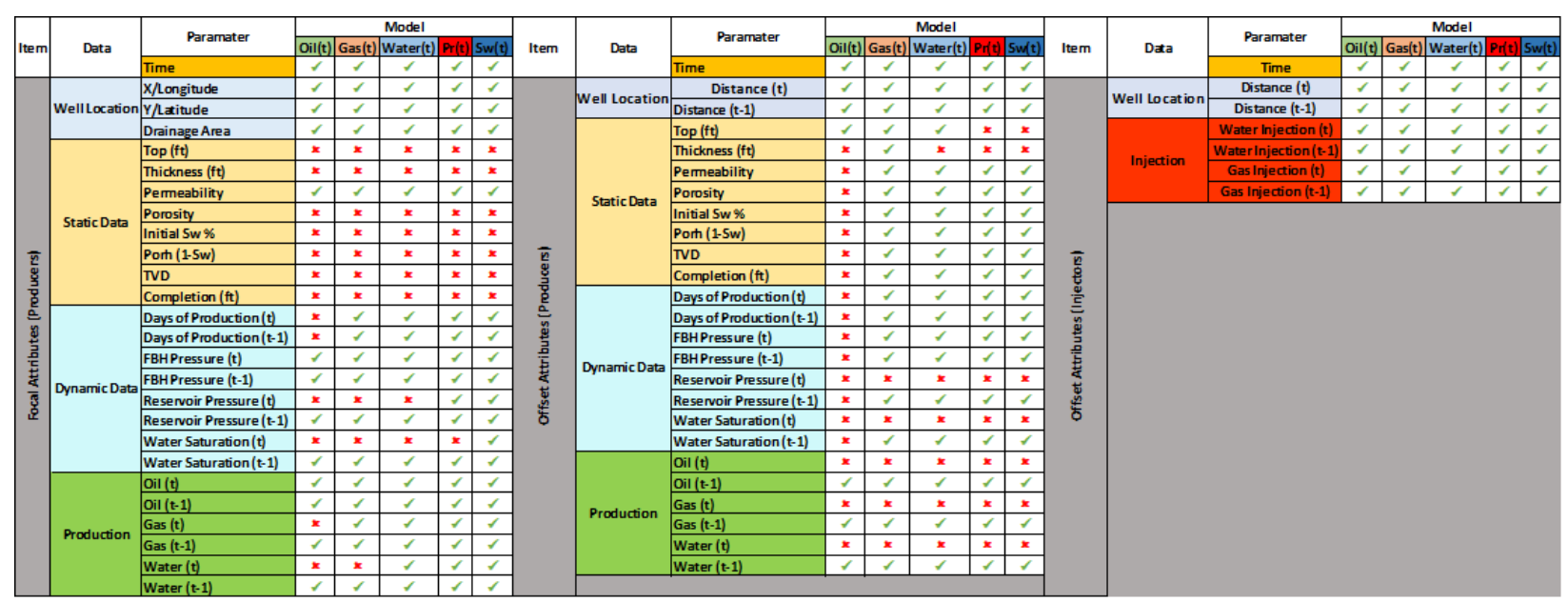

Table 5 - Attribute Selection

\begin{tabular}{|c|c|c|c|c|c|c|c|c|c|}
\hline \multicolumn{4}{|c|}{ Well Information } & \multicolumn{4}{|c|}{ Offset Wells Injector } & \multirow{2}{*}{\begin{tabular}{|l|} 
Output \\
$\mathbf{q}(t)-0$ il \\
\end{tabular}} & \multirow[t]{2}{*}{ ^ } \\
\hline Index & $\begin{array}{c}\text { Well } \\
\text { Index }\end{array}$ & $\begin{array}{l}\text { Well } \\
\text { Name }\end{array}$ & $\begin{array}{c}\text { Time } \\
\text { Index }\end{array}$ & Water Saturation [\%](t)[2]] & Water Saturation [\%][t-1][(2)] & Water_Injection(t)[2I] & Water_Injection(t-1)[2I] & & \\
\hline 237 & 28 & P.09 & $03 / 31 / 1998$ & 1.000000954 & 1.000000954 & 2000 & 2000 & 30.92 & \\
\hline 6546 & 49 & P.31 & $06 / 30 / 2013$ & 0.544372797 & 0.559739113 & 0 & 2000 & 31.06 & \\
\hline 5358 & 37 & P.19 & $06 / 30 / 2011$ & 0.939733207 & 0.94904089 & 0 & 2000 & 31.63 & \\
\hline 3749 & 26 & P.06 & $07 / 31 / 2008$ & 0.233641222 & 0.714284539 & 0 & 0 & 31.82 & \\
\hline 3175 & 28 & P.09 & $04 / 30 / 2007$ & 0.948613167 & 0.946336806 & 2000 & 2000 & 31.83 & \\
\hline 4712 & 28 & P.09 & $05 / 31 / 2010$ & 0.948292315 & 0.947067201 & 1722.661255 & 1948.028076 & 32.17 & \\
\hline 3711 & 28 & P.09 & $06 / 30 / 2008$ & 0.947219372 & 0.949728906 & 0 & 2000 & 32.42 & \\
\hline 4209 & 28 & P.09 & $06 / 30 / 2009$ & 0.946183085 & 0.949023128 & 0 & 2000 & 32.46 & \\
\hline 4648 & 62 & P.50 & $03 / 31 / 2010$ & 0.96166271 & 0.957588613 & 2000 & 2000 & 32.52 & \\
\hline 1587 & 40 & P.22 & $04 / 30 / 2003$ & 0.948512495 & 0.945686817 & 2000 & 2000 & 32.7 & \\
\hline 1902 & 25 & P.05 & $02 / 29 / 2004$ & 0.934761047 & 0.924849808 & 2000 & 2000 & 32.74 & \\
\hline 2039 & 30 & P.11 & $06 / 30 / 2004$ & 0.949011683 & 0.954115331 & 0 & 2000 & 33.03 & \\
\hline 2827 & 26 & P.06 & $06 / 30 / 2006$ & 0.713477731 & 0.727244556 & 0 & 2000 & 33.23 & \\
\hline 4663 & 28 & P.09 & $04 / 30 / 2010$ & 0.947067201 & 0.944945991 & 1948.028076 & 2000 & 33.47 & \\
\hline 1976 & 33 & P.15 & $04 / 30 / 2004$ & 1.000000954 & 1.000000954 & 2000 & 2000 & 33.58 & \\
\hline 3590 & 27 & P.07 & $03 / 31 / 2008$ & 0.999551833 & 0.999555826 & 2000 & 2000 & 34.94 & \\
\hline 1268 & 51 & P.33 & $06 / 30 / 2002$ & 0.999972105 & 0.999972224 & 0 & 2000 & 34.95 & \\
\hline 3671 & 28 & P.09 & $05 / 31 / 2008$ & 0.949728906 & 0.948237836 & 2000 & 2000 & 35.09 & \\
\hline 4614 & 28 & P.09 & $03 / 31 / 2010$ & 0.944945991 & 0.941172838 & 2000 & 2000 & 35.14 & \\
\hline 4166 & 28 & P.09 & $05 / 31 / 2009$ & 0.949023128 & 0.947511494 & 2000 & 2000 & 35.19 & \\
\hline 5986 & 28 & P. 09 & $07 / 31 / 2012$ & 0.200367019 & 0.936470807 & 0 & 0 & 35.21 & \\
\hline 1189 & 38 & P.20 & $04 / 30 / 2002$ & 0.95109272 & 0.949208677 & 2000 & 2000 & 35.37 & \\
\hline 7665 & 41 & P.23 & $05 / 31 / 2015$ & 0.940070391 & 0.937902331 & 2000 & 2000 & 35.55 & \\
\hline 1671 & 25 & P.05 & $07 / 31 / 2003$ & 0.153866515 & 0.939109147 & 0 & 0 & 35.61 & $\checkmark$ \\
\hline$<$ & & & & & & & & & $>$ \\
\hline
\end{tabular}

Table 6 - Spatiotemporal Database for Oil

Once the database had been cleaned, the total number of remaining cases was 4,679. Of those, $80 \%$ was used towards training and $10 \%$ was used respectively towards each the calibration and validation. A synopsis of the total amount of cases split into each category can be seen in Table 7 below.

\begin{tabular}{|c|c|c|c|c|}
\hline Total Cases & Imported Cases & Training Cases & Calibration Cases & Validation \\
\hline 6572 & 4679 & 3743 & 468 & 468 \\
\hline
\end{tabular}




\section{Forecasting}

After the TDM was created, (trained, calibrated and verified) and deemed to be an accurate model based on the data provided between 1995 and 2014, the deployment phase started. The deployment would determine future production of oil, gas and water for all of the wells included in the dataset. Once the predictions had been completed between January 2015 until December 2016 , the results were then to be compared to the numerical results. The final deployment type as well as the list of inputs can be seen in Figure 20 below. It is essential to add the data for the operational conditions, as seen in the figure for the years of forecasting before deployment.

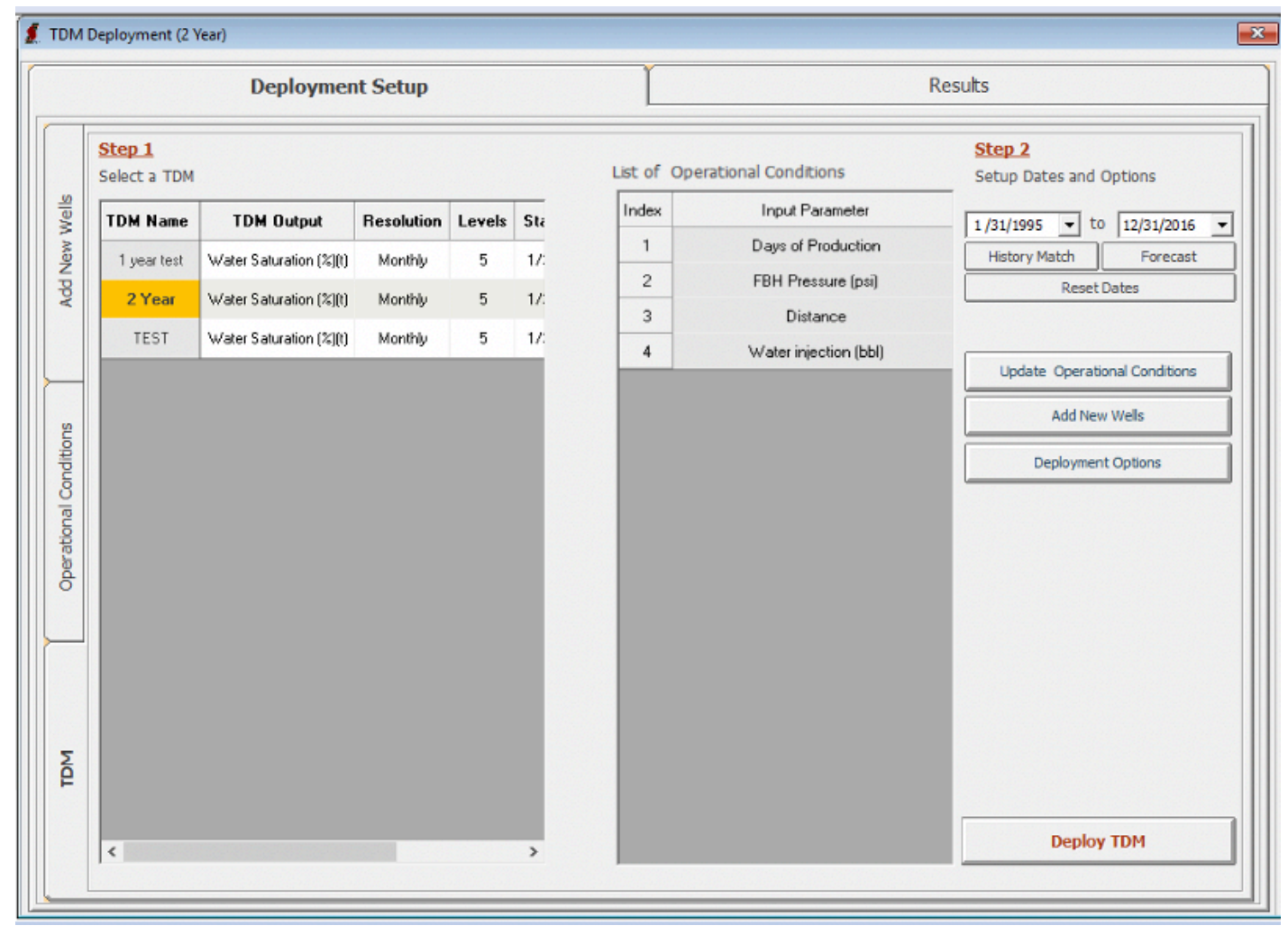

Figure 20 - TDM Deployment 


\section{CHAPTER IV}

\section{Results}

\subsection{Training Results}

The results for the training, calibration and verification of the Oil, Gas, Water, Reservoir Pressure and Water Saturation models can be found within this section. After a multitude of attempts over varying models, the best ones were selected based on their $\mathrm{R}^{2}$ training results. In order for the model to be deemed satisfactory, it needed to have a high $\mathrm{R}^{2}$ across its training, calibration and verification. If the model performed well in 1 or 2 of the three classes, but not all three the attributes were reevaluated, then the model was built and trained again.

When analyzing the results from these training sets, as seen in Figure 21 and Figure 22 below, we can see that the results for each of the models mentioned performed very well across its training, calibration and verification stages. Due to the results, these models would then be used in the final construction of the TDM. The numerical results of the $\mathrm{R}^{2}$ associated with training of each model can be seen in Table 8 below.

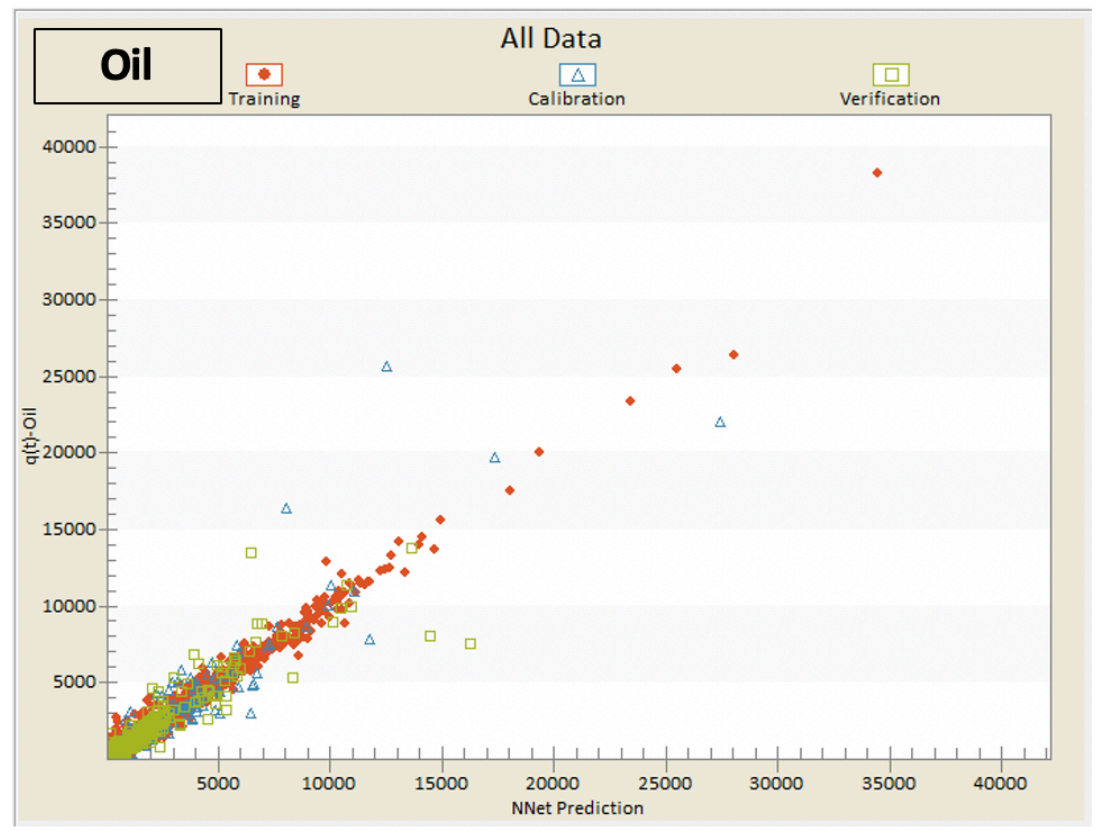

Figure 21 - Oil Training Results 

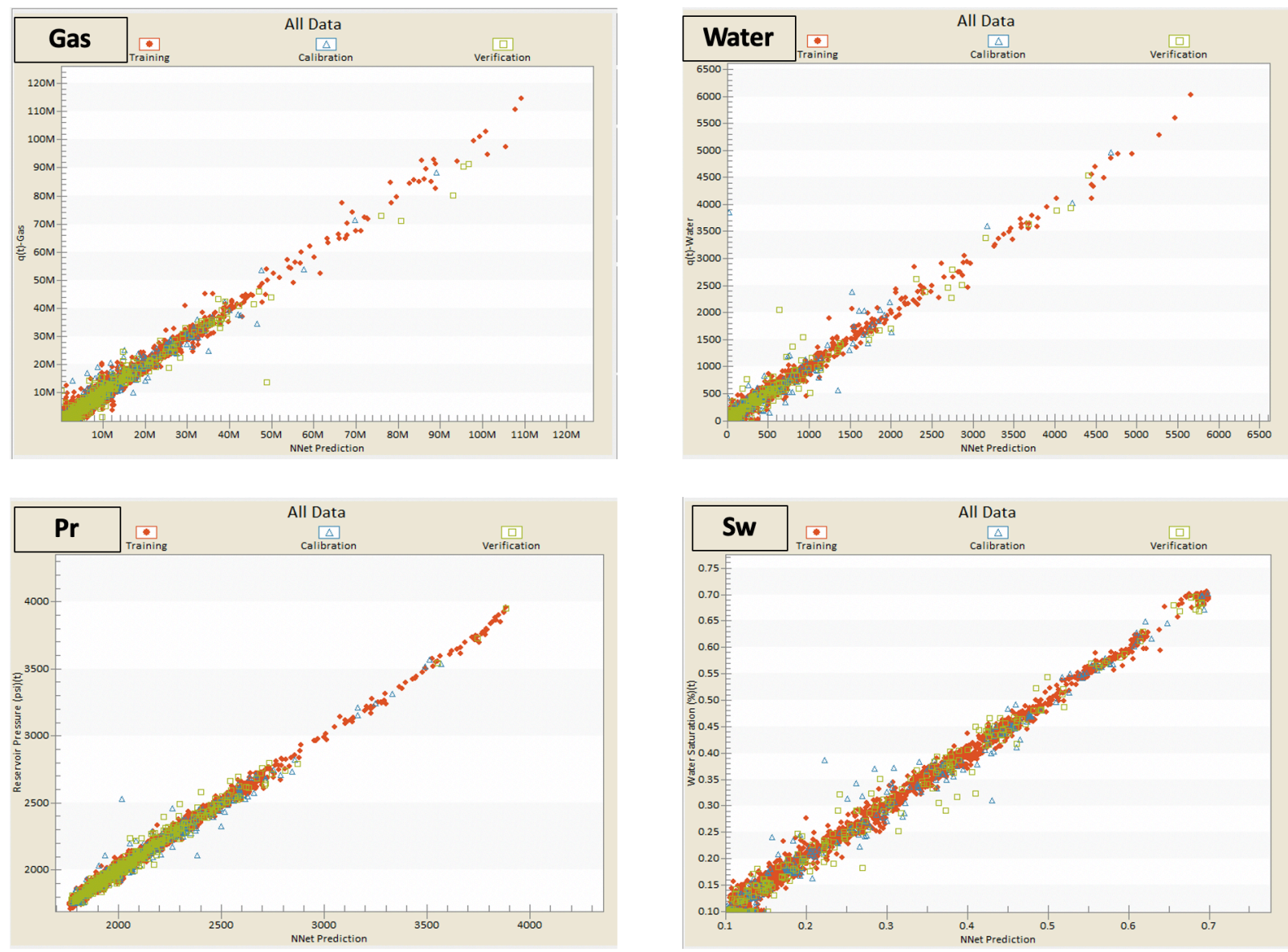

Figure 22 - Gas, Water, Pr, Sw Training Results

\begin{tabular}{|c|c|c|c|c|}
\hline \multirow{6}{*}{ 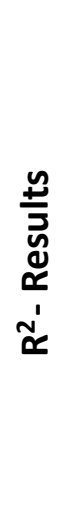 } & Model & Training & Calibration & Verification \\
\hline & Oil & 97.79 & 86.44 & 84.26 \\
\hline & Gas & 97.60 & 95.65 & 97.78 \\
\hline & Water & 96.86 & 85.0 & 95.78 \\
\hline & $\mathrm{Pr}$ & 98.97 & 97.37 & 97.82 \\
\hline & Sw & 99.40 & 98.60 & 98.76 \\
\hline
\end{tabular}

Table 8 - Numerical Training Results 


\subsection{TDM Results and Conformation}

The results from deploying the TDM can be seen in figures ranging from Figure $23-24$ below. Each of these sections of figures contains a detailed series of plots depicting how a selected well performed within the TDM. In each instance, there are three graphs displaying how the Oil, Water and Gas production performed for said wells. Among all of the provided graphs, the $X$-axis can be seen to be time whereas the $Y$ axis is the volume alternating between barrels for oil and water or mscf for gas. Within these results, you can see the accuracy of the training sections as well as the forecasting. The division between the two sections is indicated by way of the vertical line at the 2015 time mark. The green, orange or blue dots represent oil, gas and water respectively, showing the results obtained from the numerical simulation. The grey line represents the training and forecasting of the completed TDM. Also, overlaid within the figures is a cumulative production calculated for each well. The cumulative for the actual matches the color of the actual data points within the given attribute being displayed, whereas the lighter green is the cumulative of the TDM's predictions. From these results, it can be concluded that the TDM is a very powerful technique when it comes to history matching the results across the given measured data for each well within the given field. It has been illustrated within Dr. Mohaghegh's book (Mohaghegh, Data-Driven Reservoir Modeling, 2017), that top-down

modeling learns the reservoir and independent wells production behavior by way of determining internal correlations within the static and dynamic datasets provided to it. Along with this, it takes into account the production of each individual well and the injection and production of offset wells. The TDM will find the patterns within the data on a well-by-well scenario, looking at each individual record given to it and then cumulatively come to a concise conclusion about the entirety of the reservoir as seen in Figure 28 through Figure 30. The accuracy of this TDM is impressive when looking at the history matching section alone not to mention when looking over how well it forecast the data from the numerical simulation based on the data provided to it for training. 


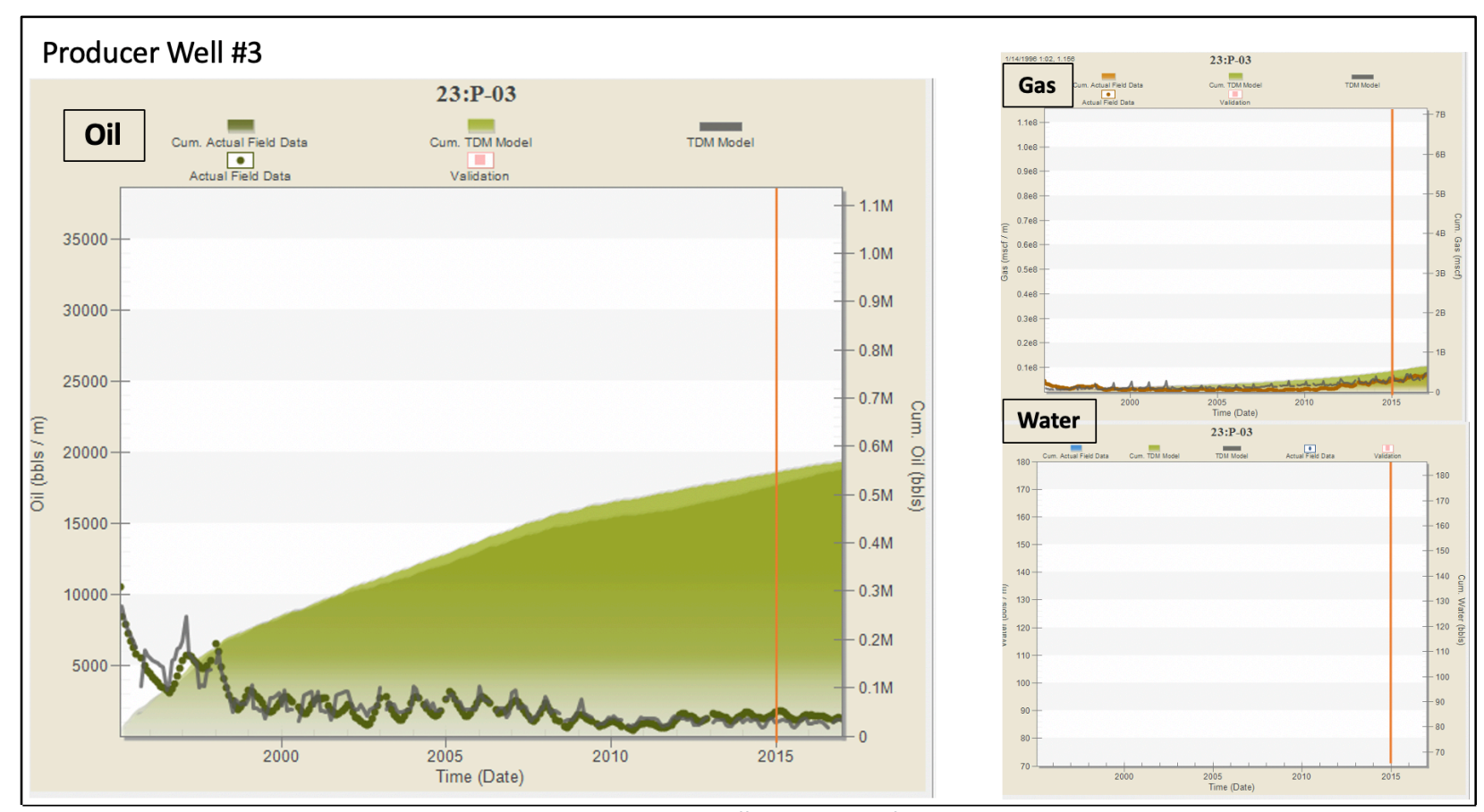

Figure 23 - Well 3 TDM Results

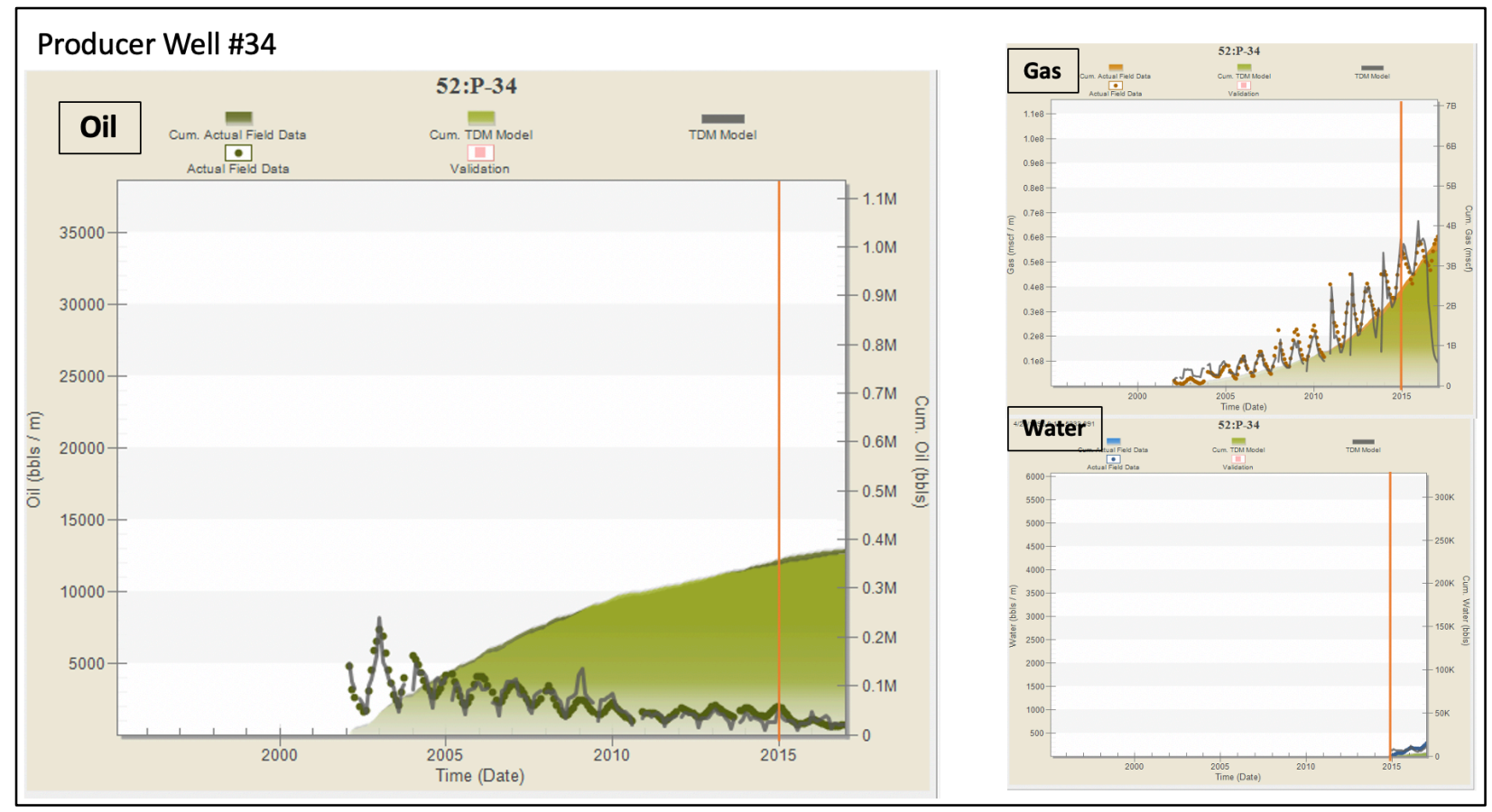

Figure 24 - Well 34 TDM Results 


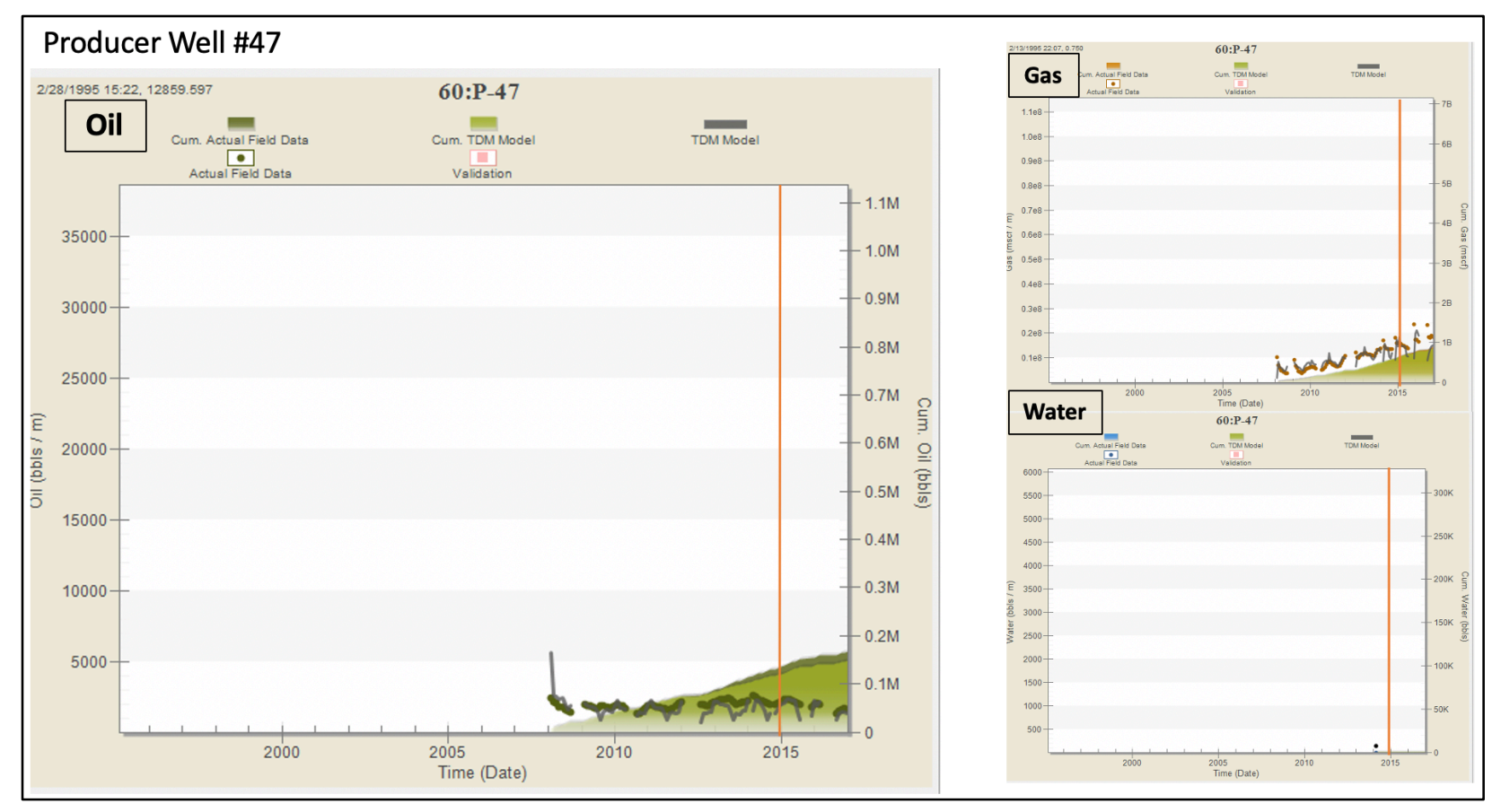

Figure 25 - Well 47 TDM Results

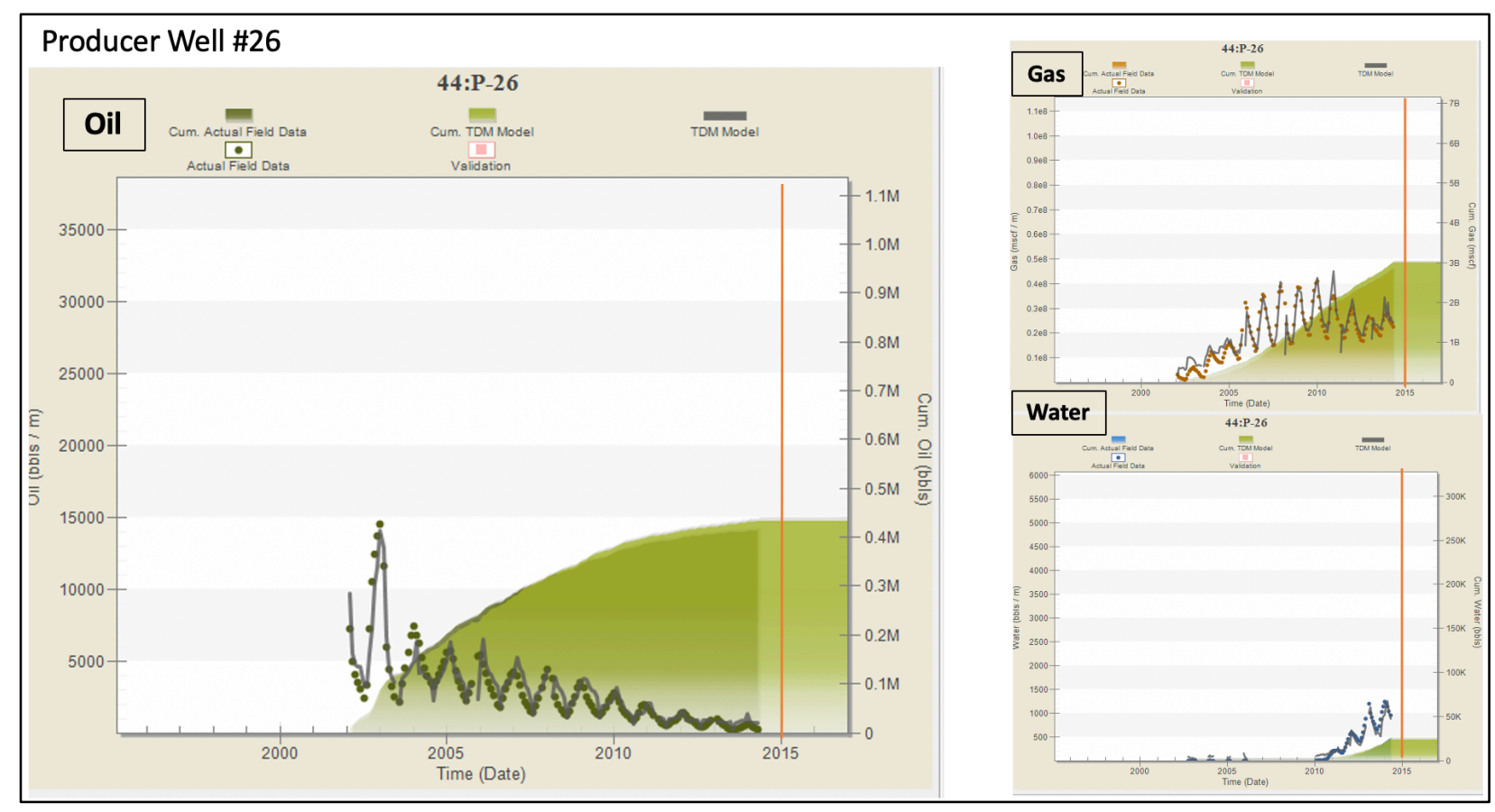

Figure 26 - Well 26 TDM Results 


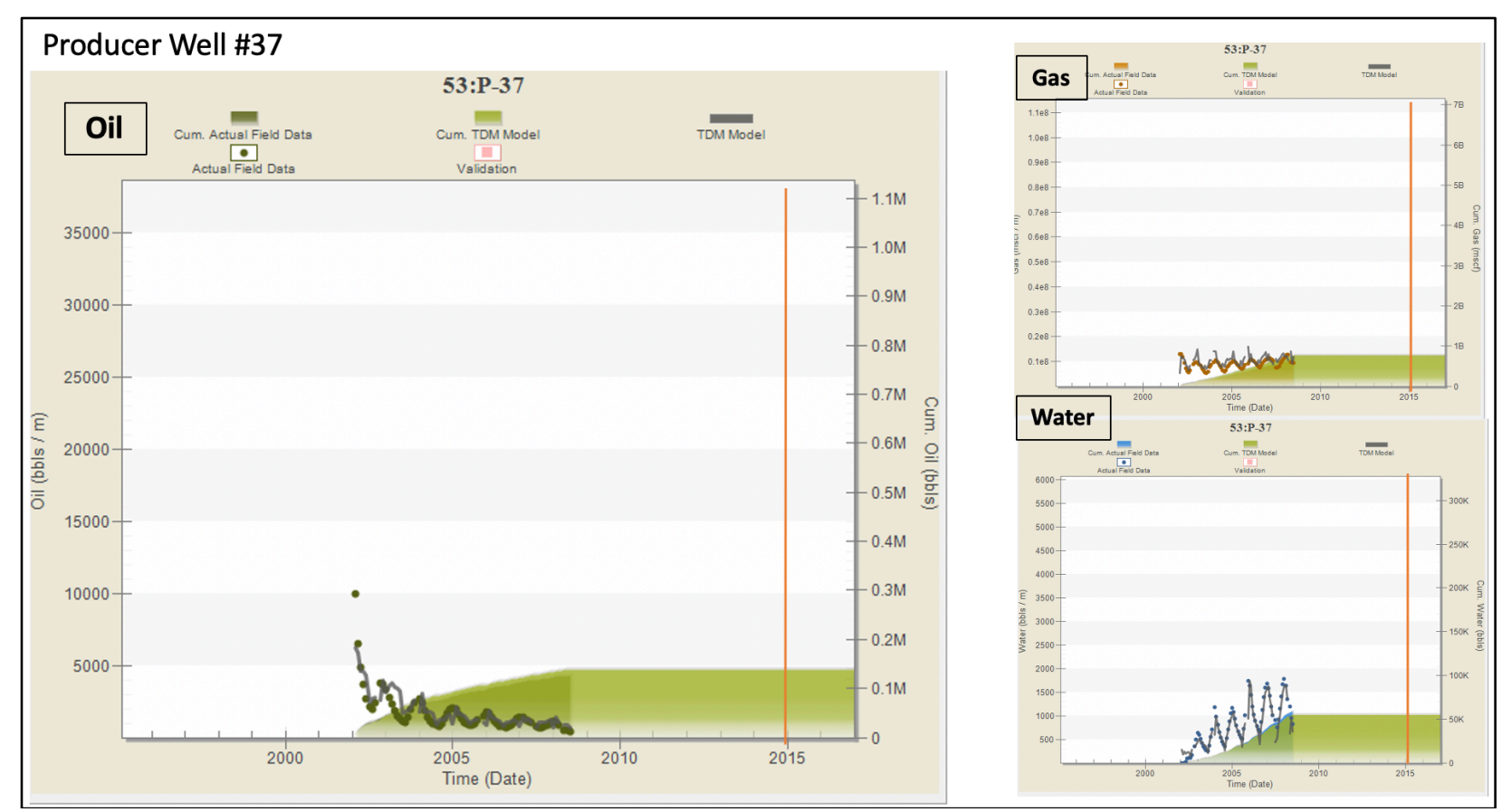

Figure 27 - Well 37 TDM Results

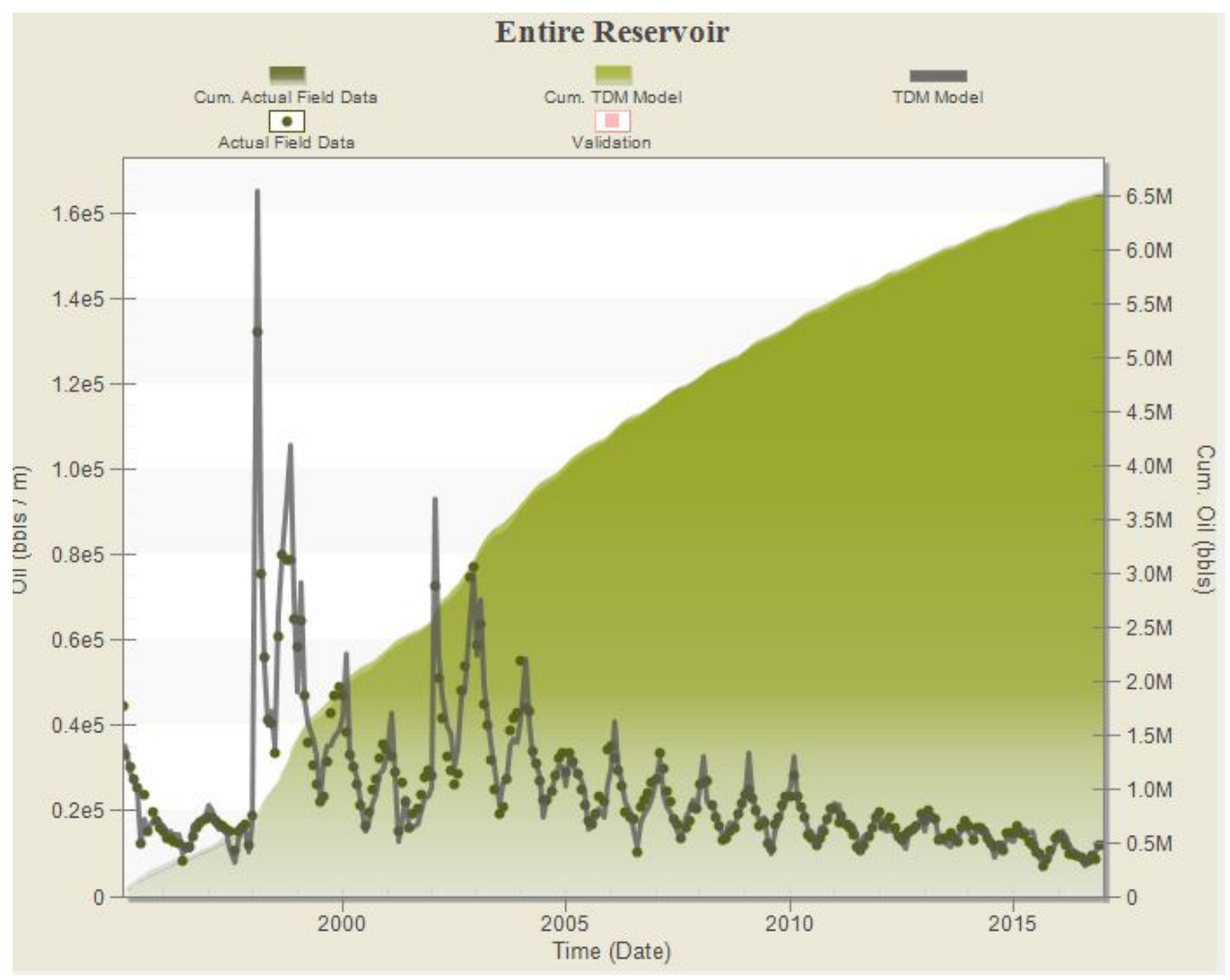

Figure 28 - Oil Production Entire Reservoir 


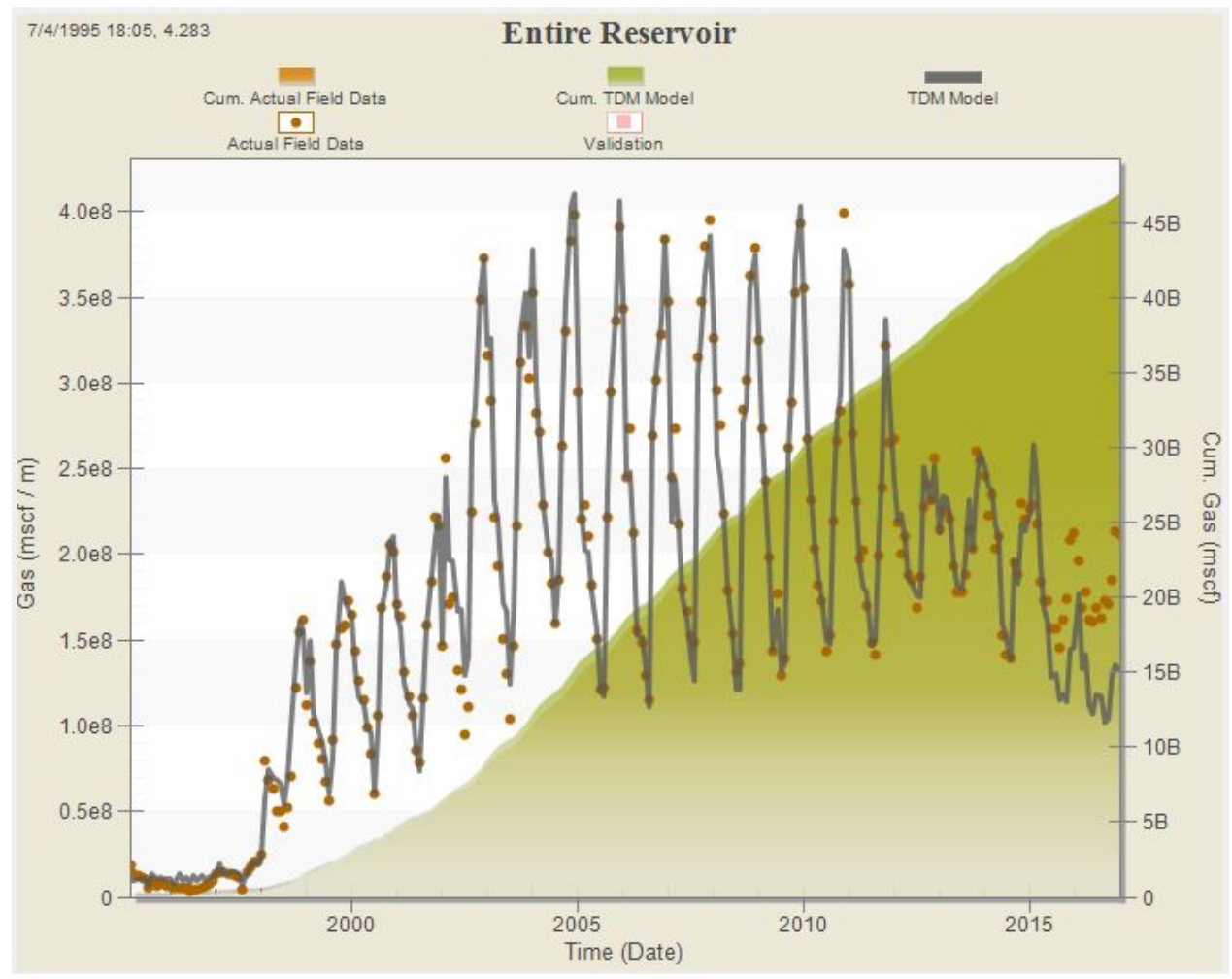

Figure 29 - Gas Production Entire Reservoir

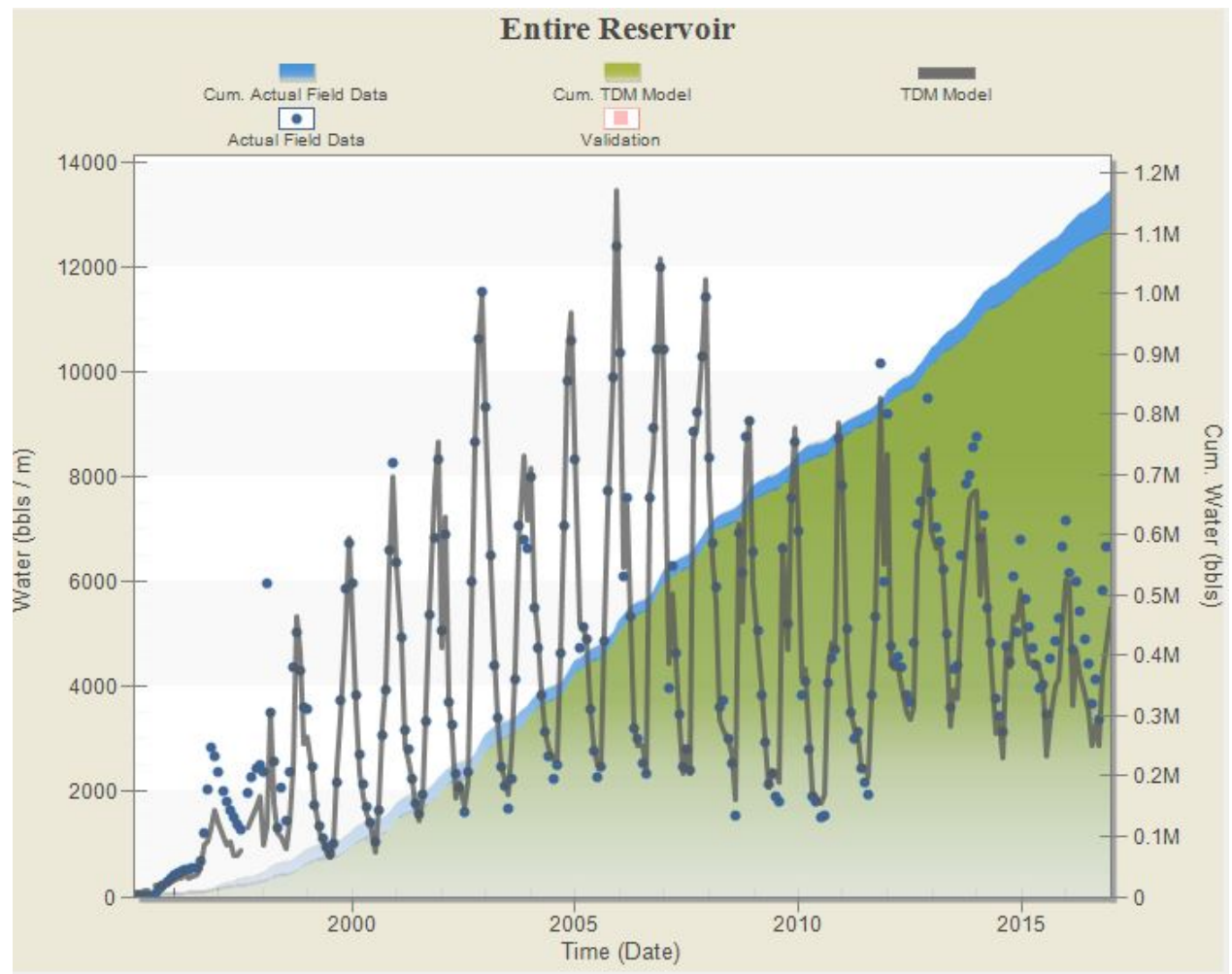

Figure 30 - Water Production Entire Reservoir 
Subsequently, the prediction results or the reservoir pressure as well as the water saturation were compared to those of the initial numerical reservoir simulator to validate the accuracy of the model. In order to visualize these results they a heat mapping approach was implemented. This was done by taking the known values for each well and radially averaging them to generate the heat map. All values used were for an entire well, the values form the numerical simulator were averaged while weighted with height of the reservoir layer as well as the permeability. A graph was generated for each attribute, reservoir pressure and water saturation, at each month of the forecasting period. In Figure 31 and Figure 32 below a summary of the initial time step for the last 3 years of production can be seen. The first image shows the last year of training and the latter two are both in forecasting to showcase how accurate the model was in training and how the further out the forecasting is deployed the model retains the ability to accurately forecast reservoir characteristics.

Reservoir Pressure (psi) at 2014-01-31
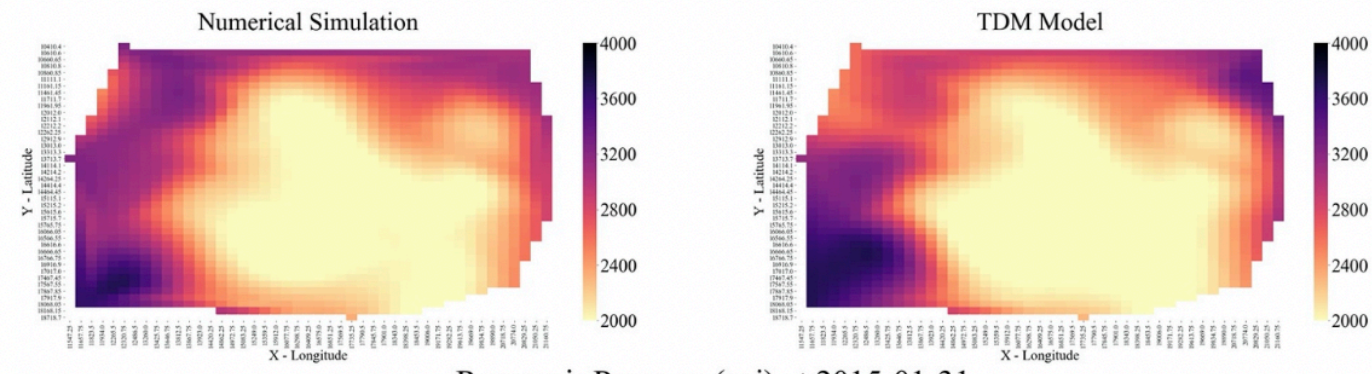

Reservoir Pressure (psi) at 2015-01-31
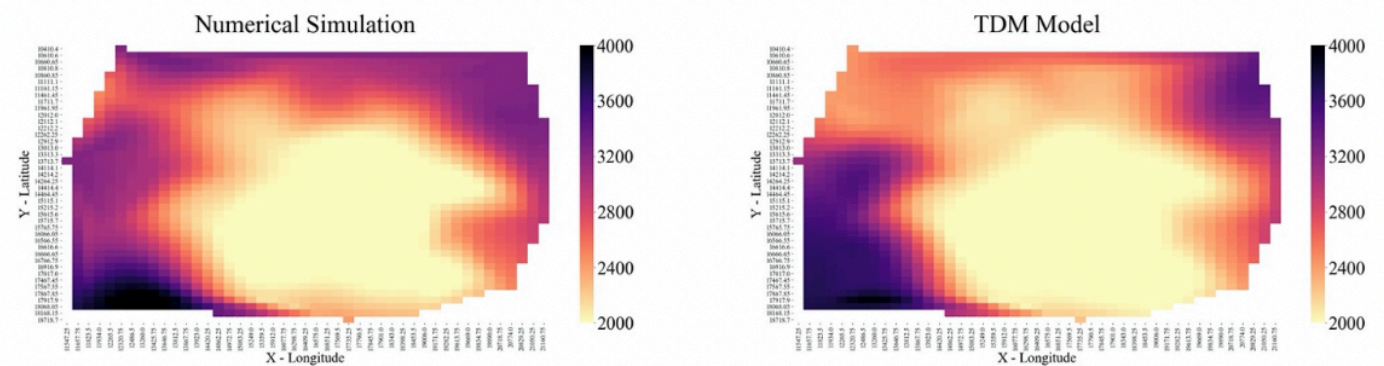

Reservoir Pressure (psi) at 2016-01-31
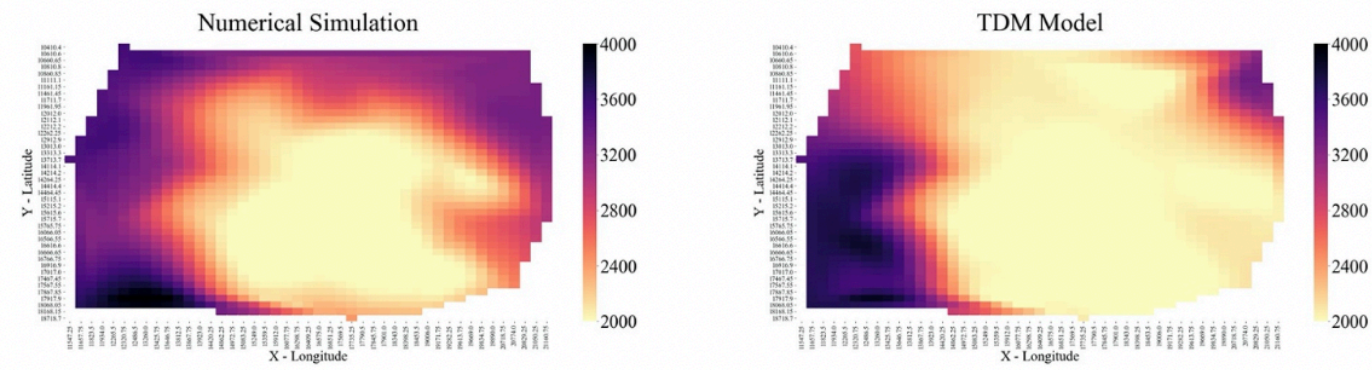

Figure 31 - Reservoir Pressure Forecasting Heat Map Summary 
Water Saturation (\%) at 2014-01-31
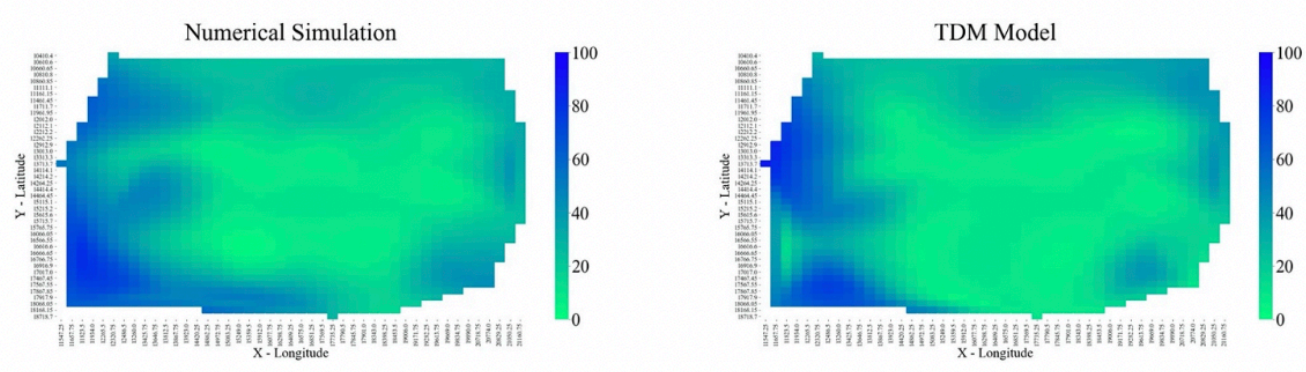

Water Saturation (\%) at 2015-01-31
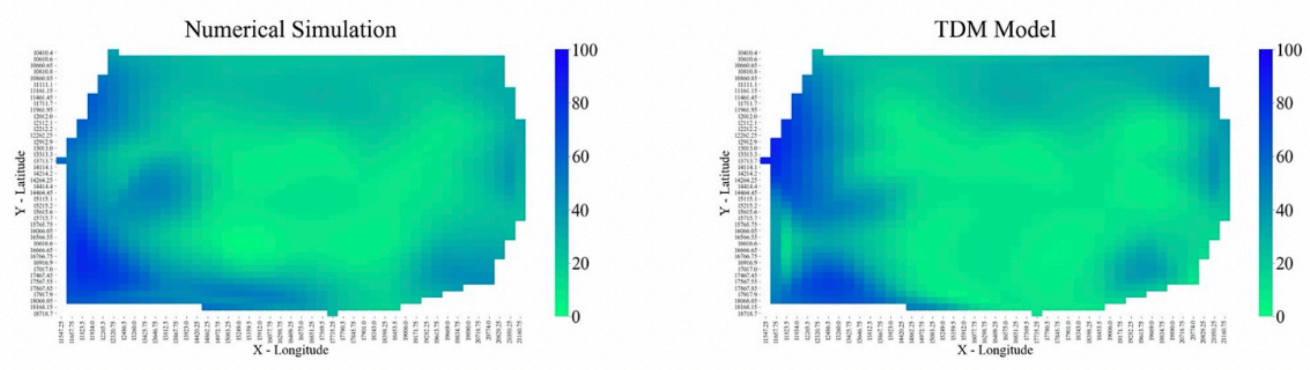

Water Saturation (\%) at 2016-01-31
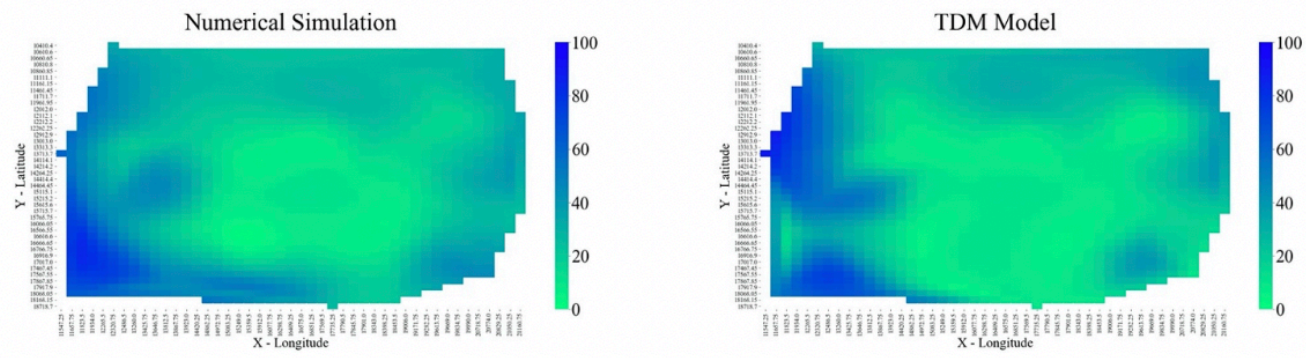

Figure 32 - Water Saturation Forecasting Heat Map Summary 


\section{CHAPTER V}

\section{Conclusion}

\subsection{Conclusion}

The operation of a reservoir is only as successful as your ability to model and understand the reservoir in question. The current most widely accepted technique for this is numerical simulation approaches. However, when considering this technology, it cannot be considered to be truly an accurate representation of the reservoir due to the amount of collected data it omits as well as the amount of interpretation that is needed to be completed to build a model. Therefore, a data-driven reservoir modeling technique, also known as Top-Down Modeling (TDM), provides not only a more comprehensive, but also a more complete modeling approach than traditional numerical reservoir simulations. This is due to its inclusion of the recorded field data while not making interpretations based on assumptions. As a result, it makes TDM an extremely appropriate tool for reservoir modeling in the existing field and determines the future performance of the field as opposed to traditional approaches.

Due to the amount of criticism that TDM is subjected to for not using a physics-based approach of modeling, synthetic data was generated from the entrusted existing technique. This data was then used to display the capabilities of a data-driven reservoir modeling approach, thus confirming the overall potential of TDM technology.

The TDM presented was trained, calibrated and validated based on the historical data given to it. This data was generated by way of a complex numerical reservoir simulation model that included a myriad of intricacies; Some of which include compartmentalization, nonproducing layers, alternating injection fluids as well as dynamic completions of wells. The results prove that TDM is a powerful technology for history matching an existing field based on collected field data for each well. Moreover, predictions were made based on the data presented to the final TDM and compared to that of the Numerical Reservoir Simulation. It was clearly shown that the results of the TDM's predictions over the final two years of the reservoir (2015 and 2016) for oil, gas and water production were directly comparable to those of the numerical simulation 
model. Therefore, it can be said that the data-driven reservoir modeling approach using a combination of artificial intelligence as well as machine learning is a justifiable approach to reservoir modeling. It can be trusted for and applied to highly complex reservoirs only the actual measured data from the field and not making any interpretations. 


\section{Bibliography}

Mohaghegh, S. D. (2011, January). Reservoir Simulation and Modeling Based on Pattern

Recognition. In SPE Digital Energy Conference and Exhibition. Society of Petroleum Engineers.

Mohaghegh, S. D. (2017). Data-Driven Reservoir Modeling. Society of Petroleum Engineers.

Mohaghegh, S. D. (2017). Shale Analytics. Springer, Cham.

Mohaghegh, S. D., Al-Mehairi, Y., Gaskari, R., Maysami, M., \& Khazaeni, Y. (October, 2014). Data-driven reservoir management of a giant mature oilfield in the Middle East. In SPE annual technical conference and exhibition. Society of Petroleum Engineers.

Shahkarami, A., Mohaghegh, S. D., Gholami, V., \& Haghighat, S. A. (2014, April). Artificial Intelligence (AI) Assisted History Matching. In SPE Western North American and Rocky Mountain Joint Meeting. Society of Petroleum Engineers . 


\section{Appendix}

\section{CMG Results}

Grid Top (ft) 2010-07-01

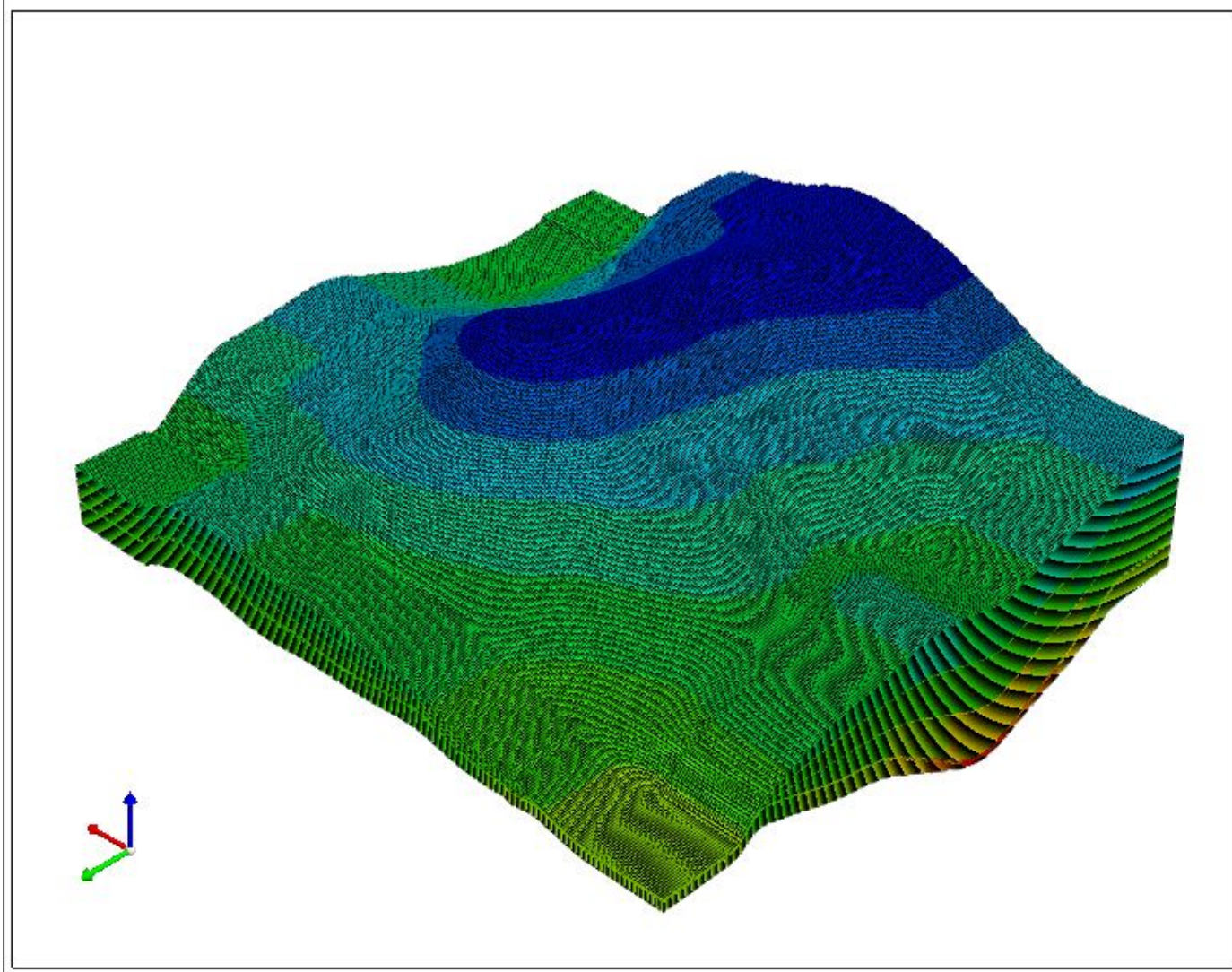

File: Final All Laye

User: awmorrow

Date: $4 / 1 / 2020$

ZX: 9.00:1

4,613
4,541
4,470
4,398
4,326
4,255
4,183
4,112
4,040
3,968
3,897




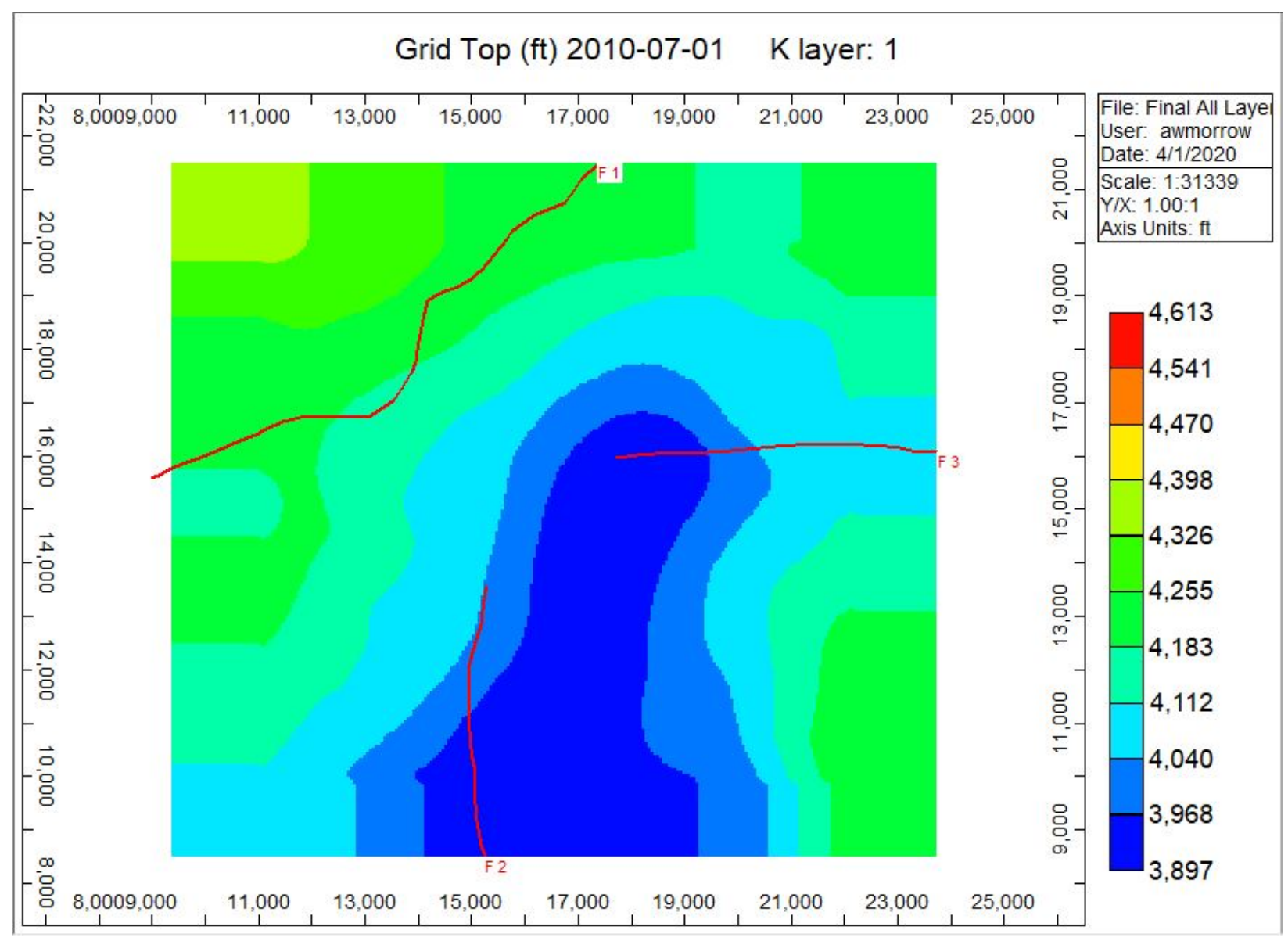




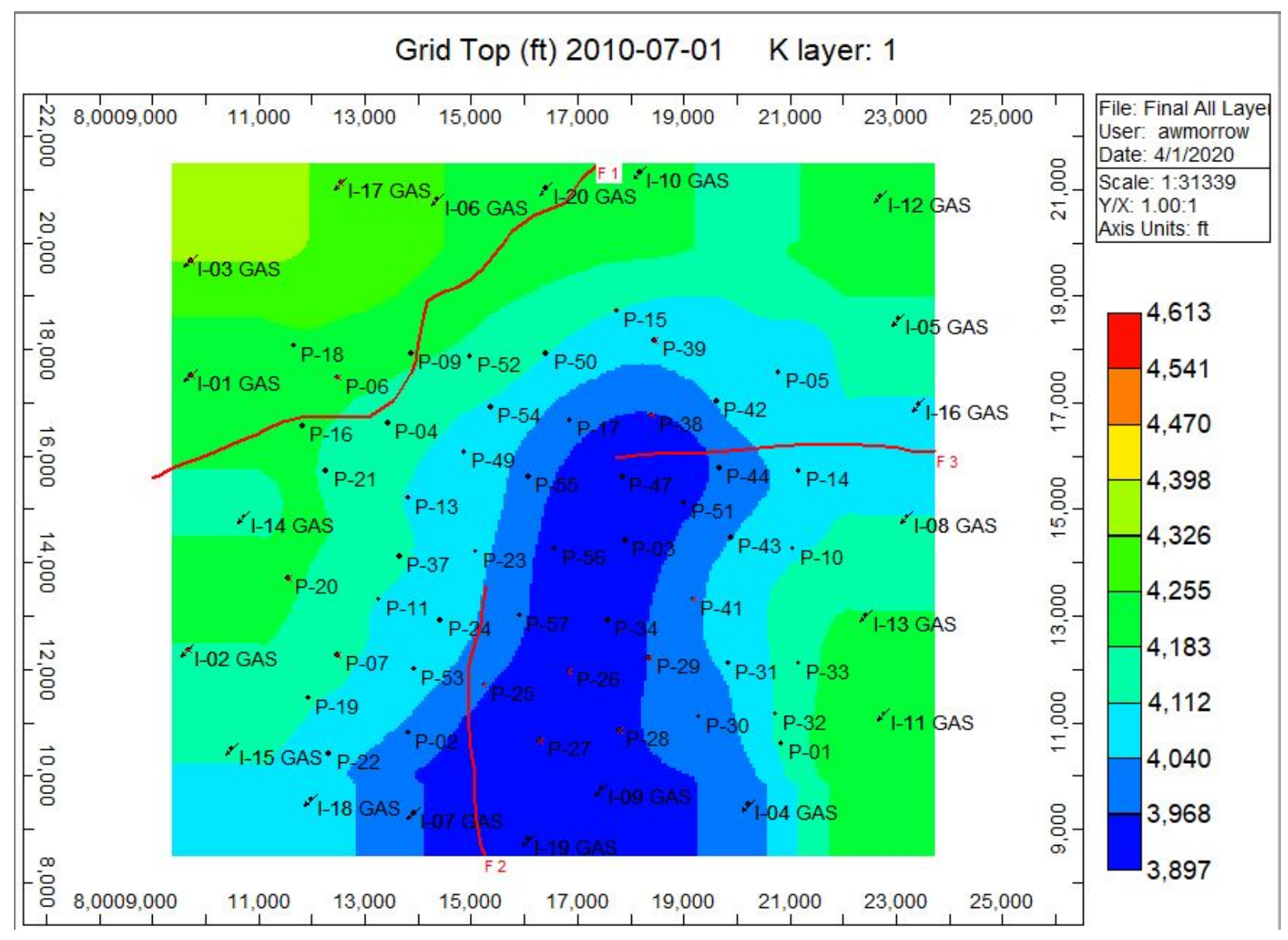


TDM Results

Oil Results

Oil: P-01

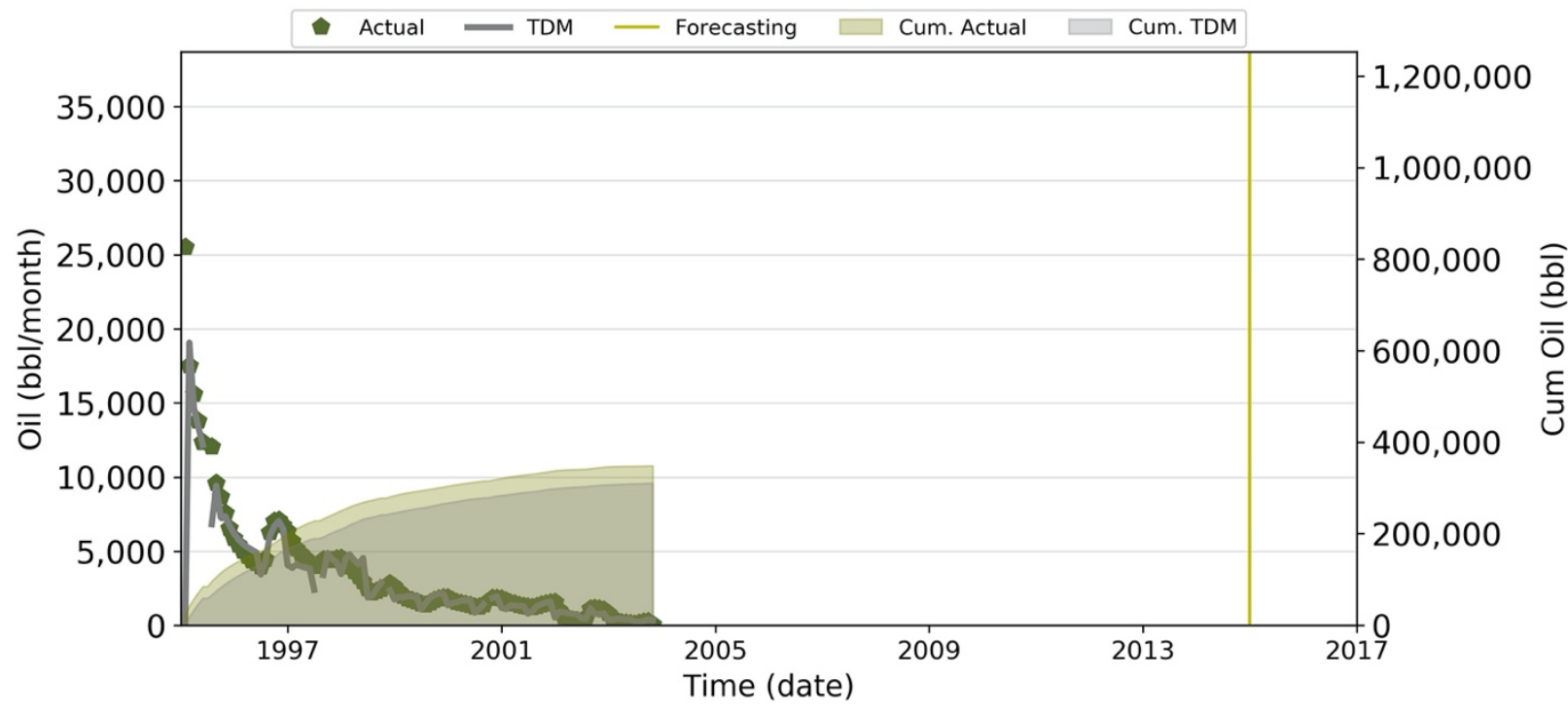

Oil: P-02

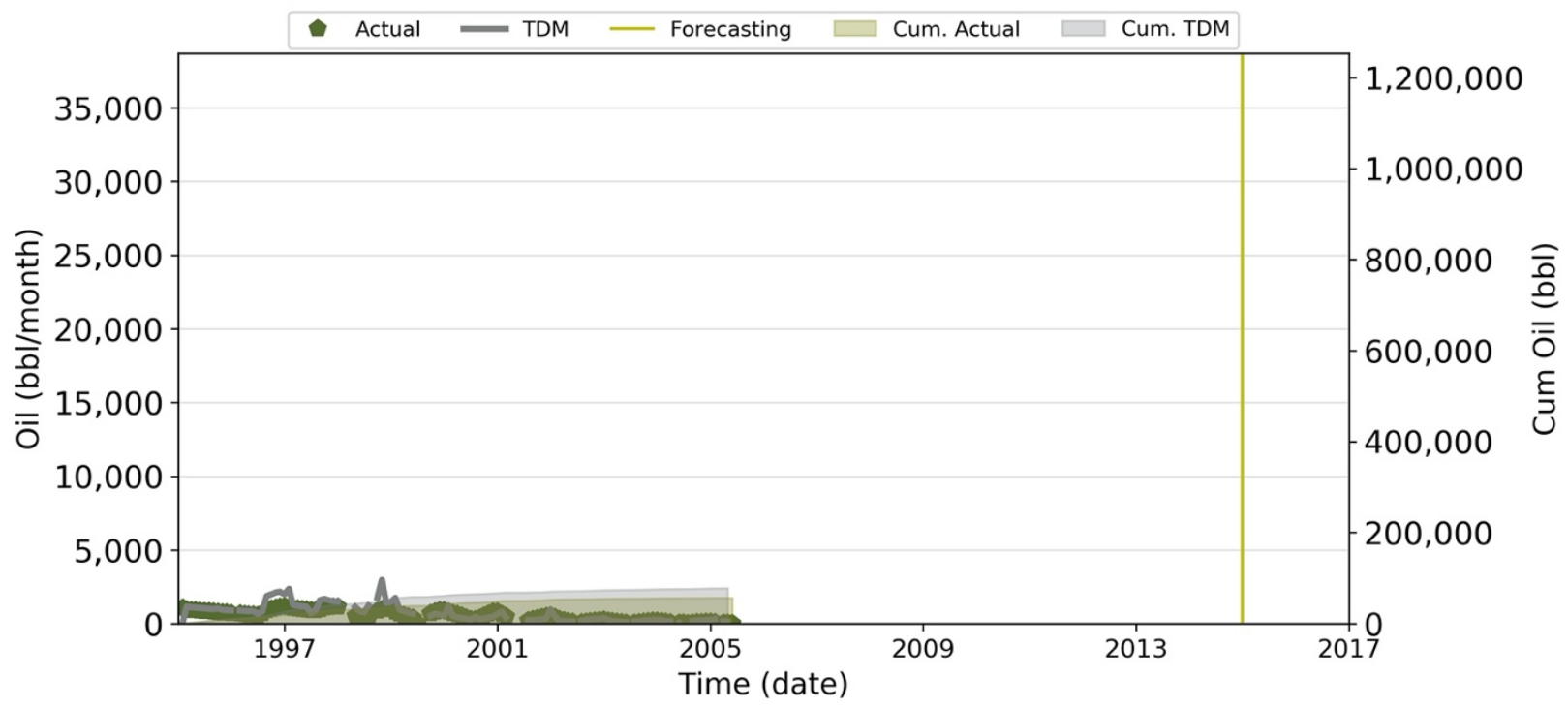


Oil: P-03

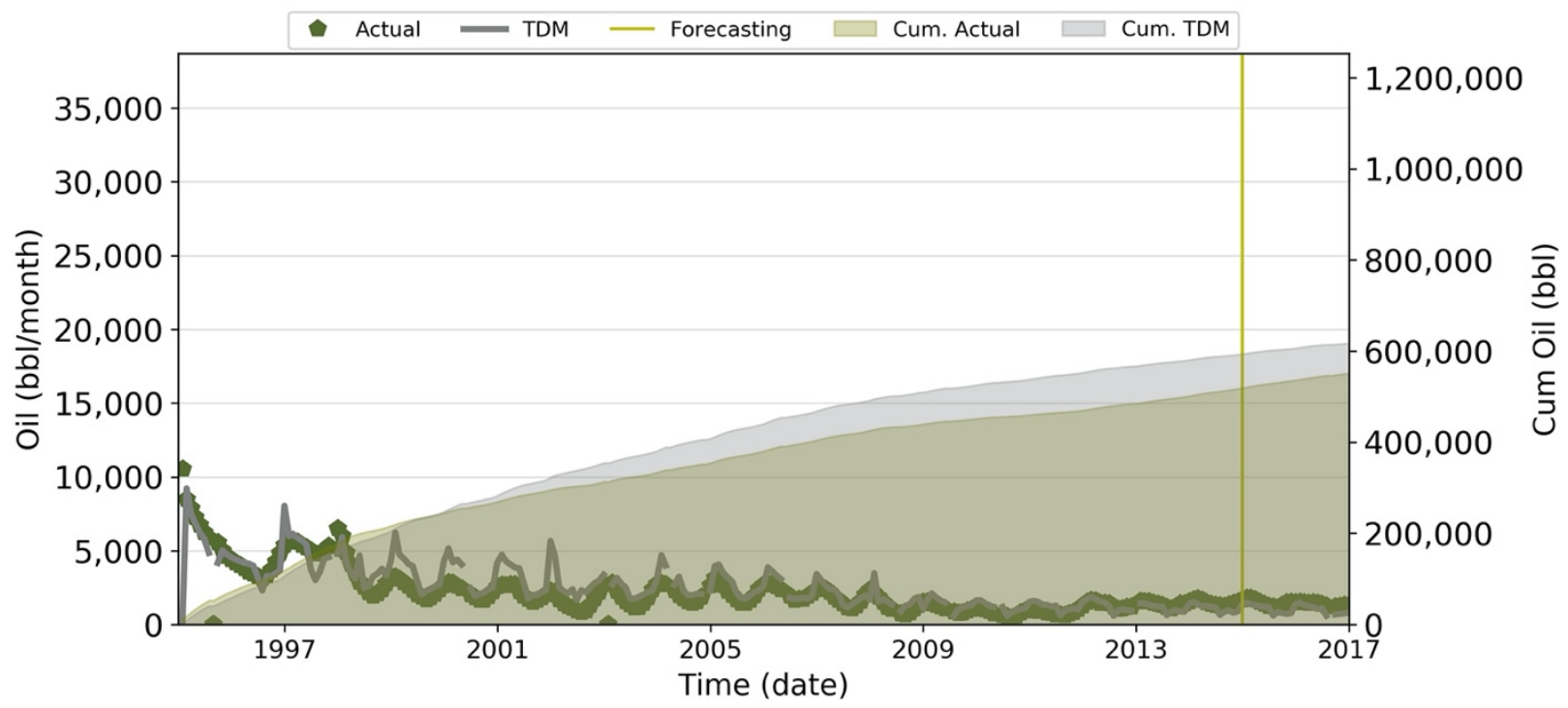

Oil: P-04

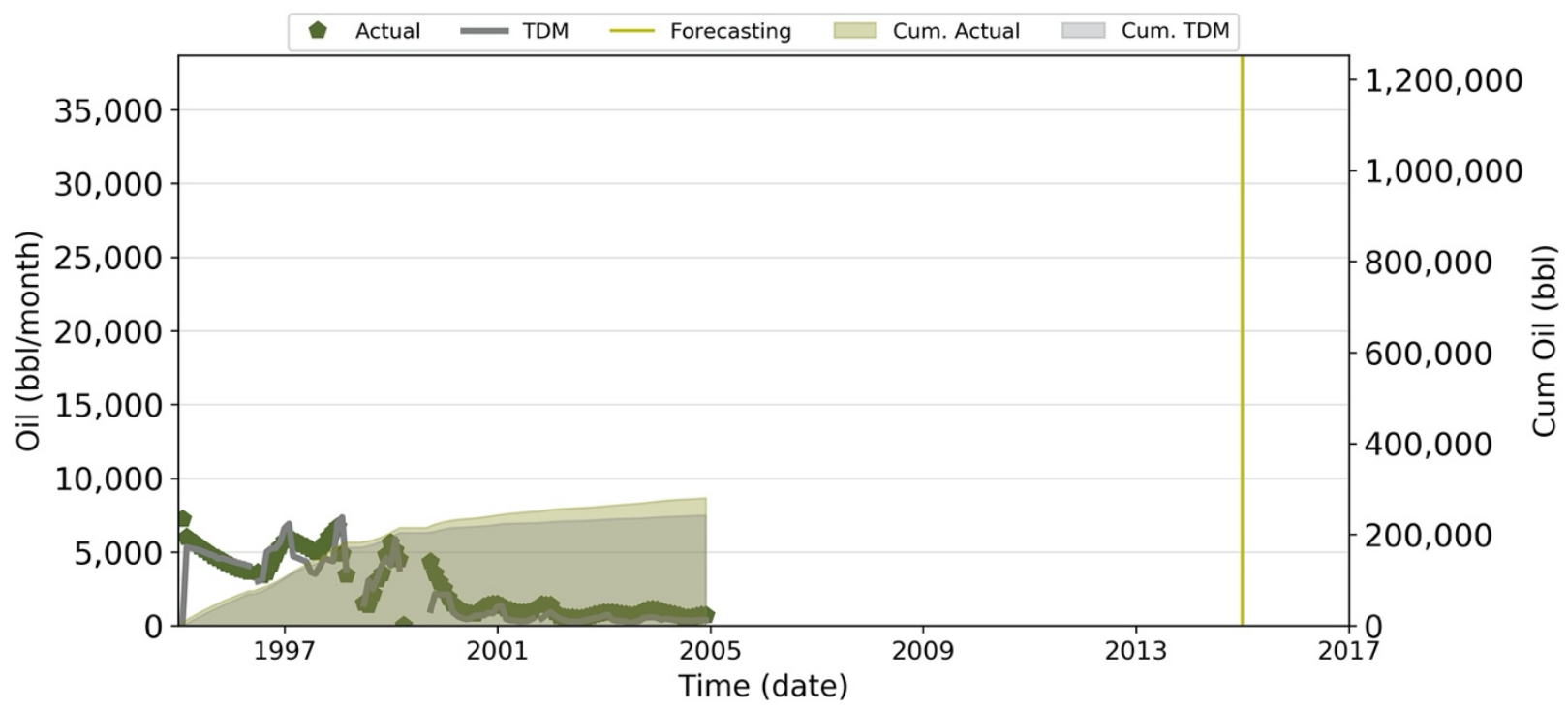


Oil: P-05

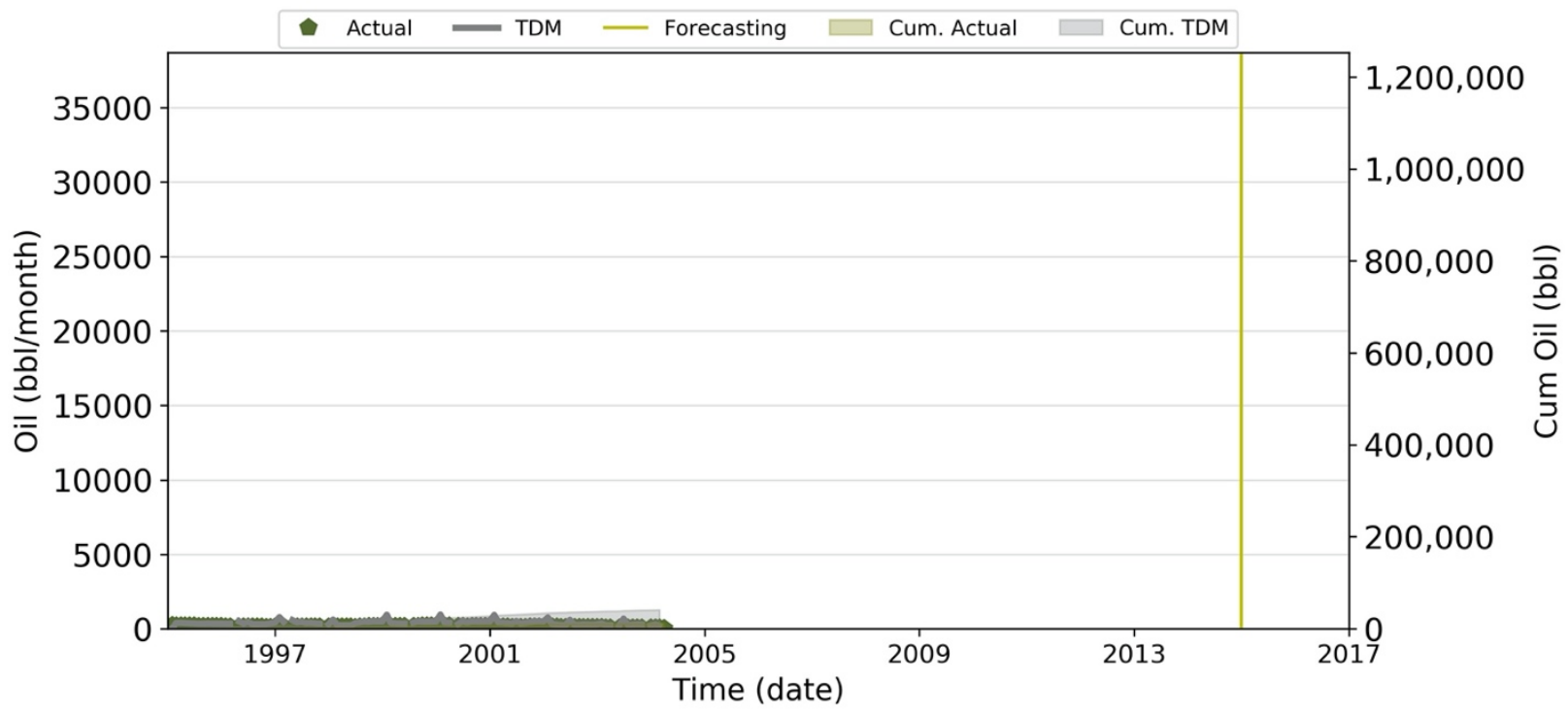

Oil: P-06

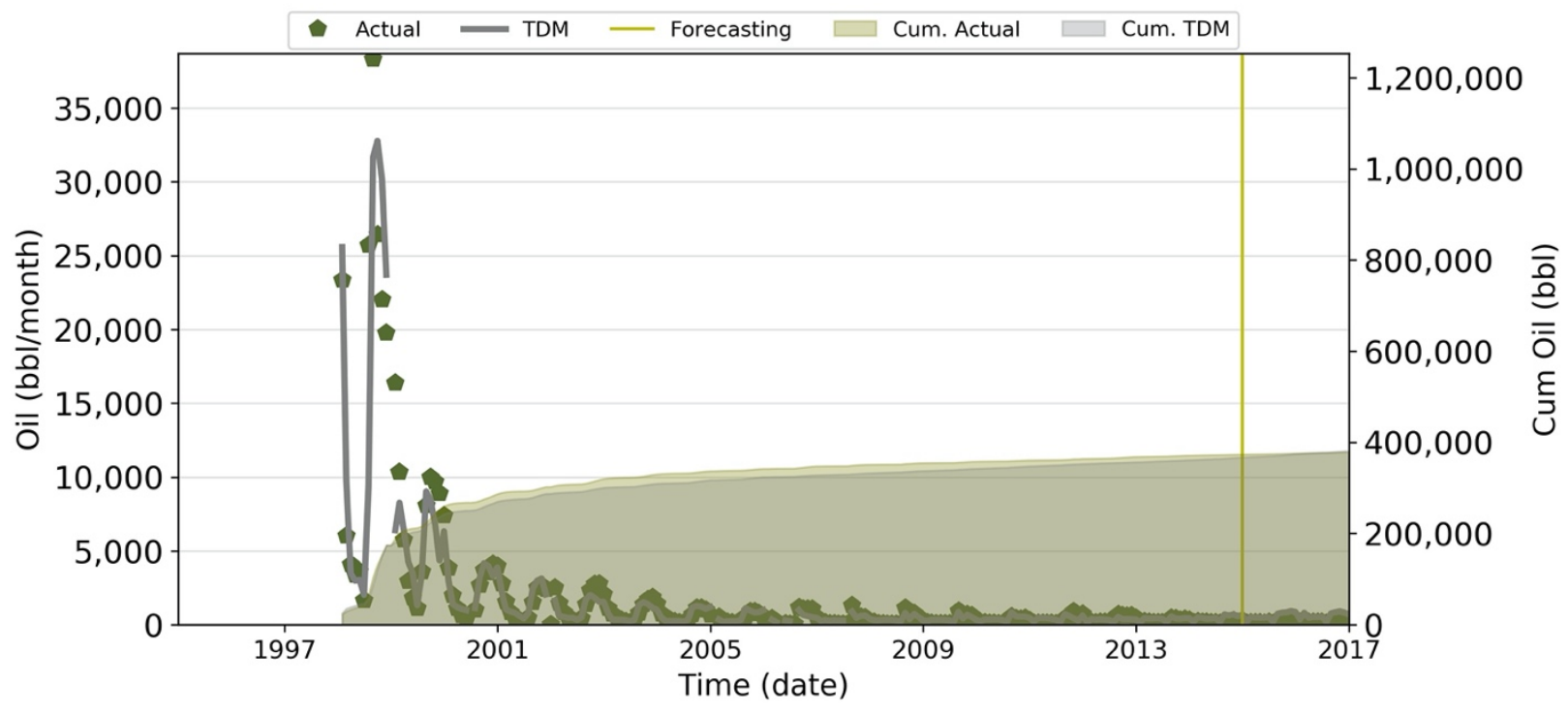


Oil: P-07

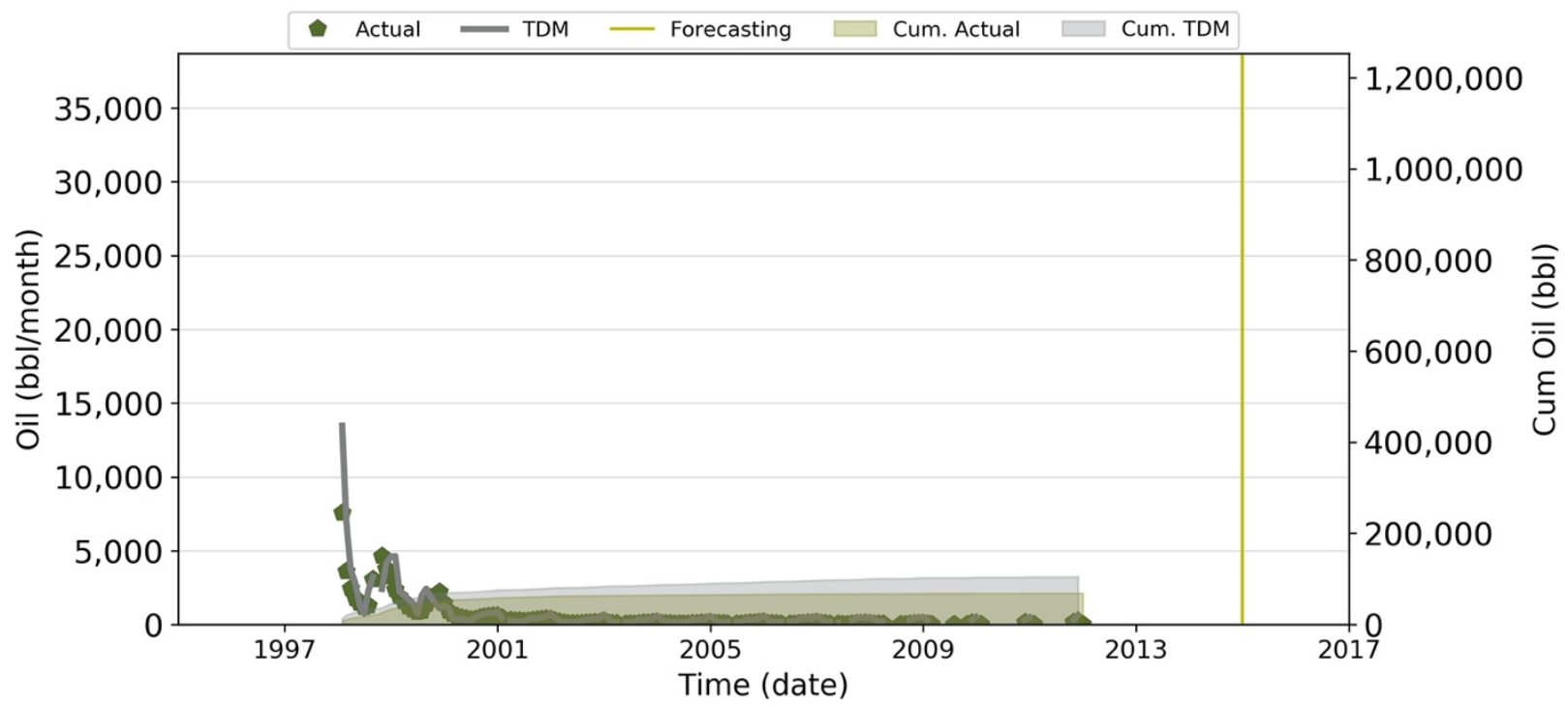

Oil: P-09

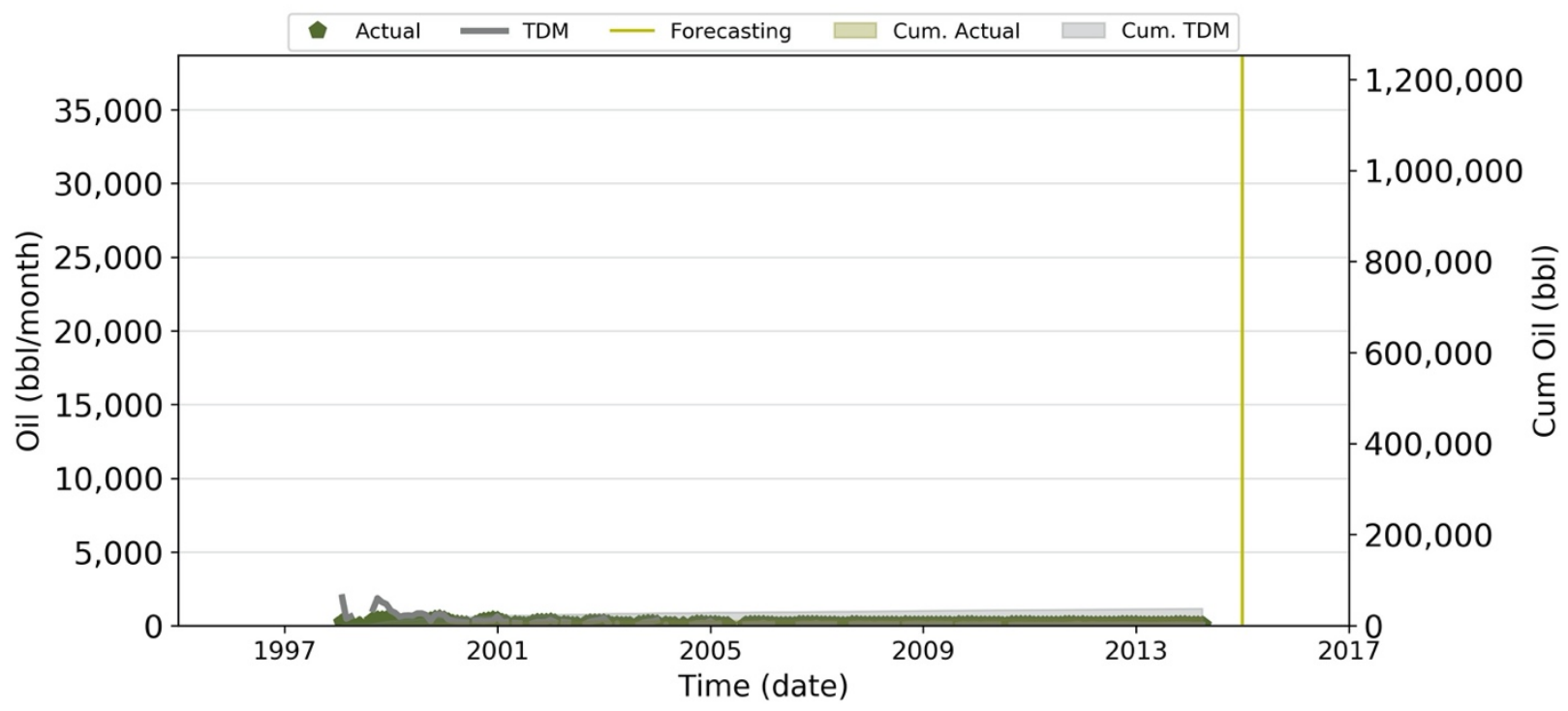


Oil: P-10

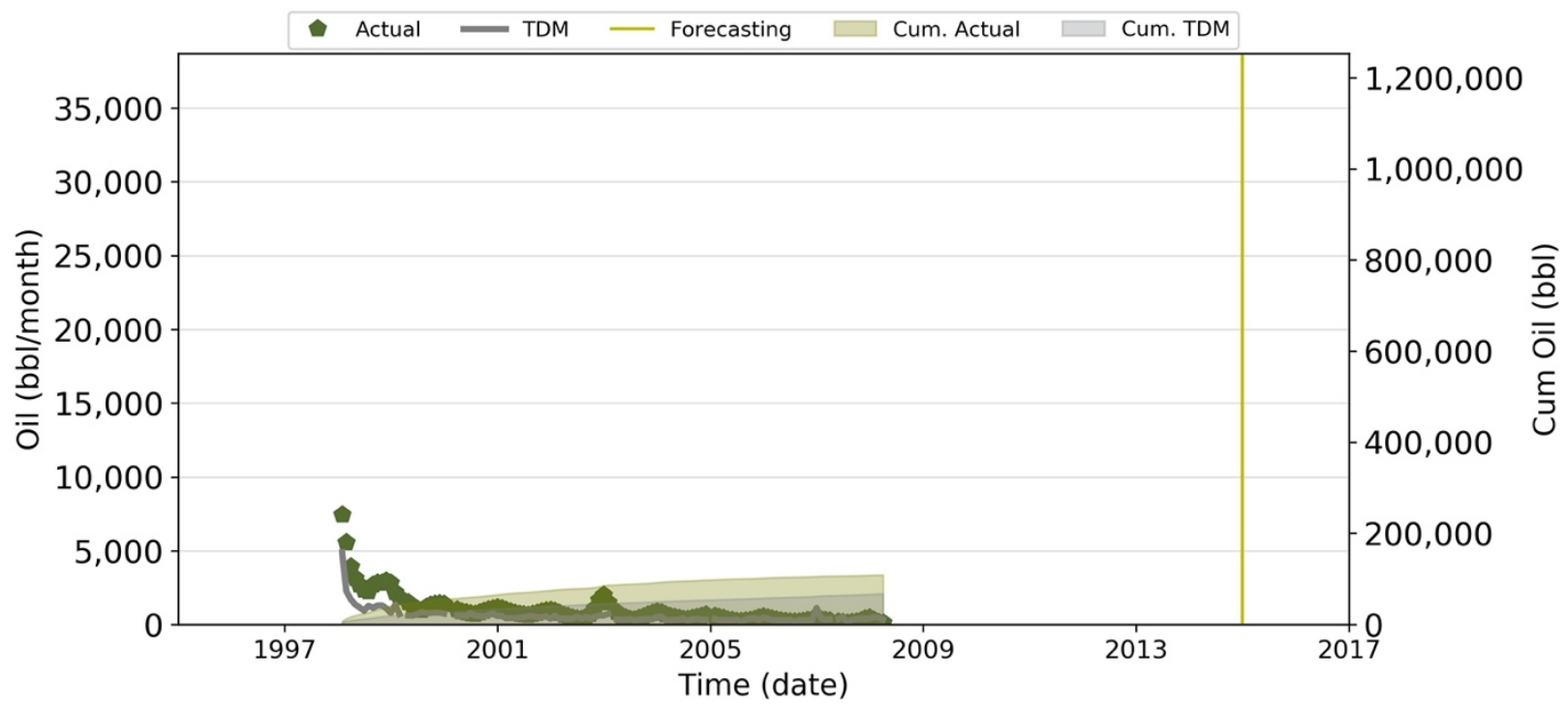

Oil: P-11

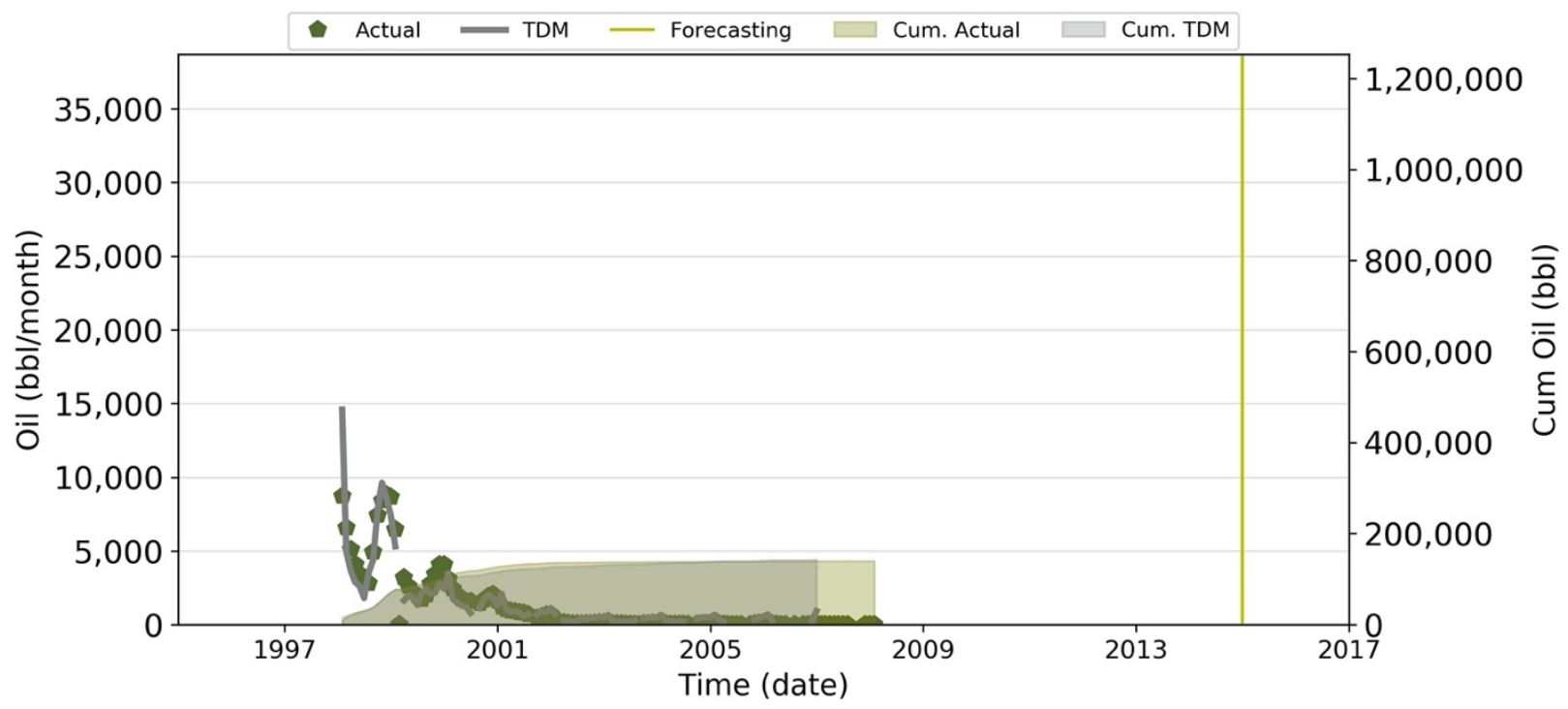


Oil: P-13

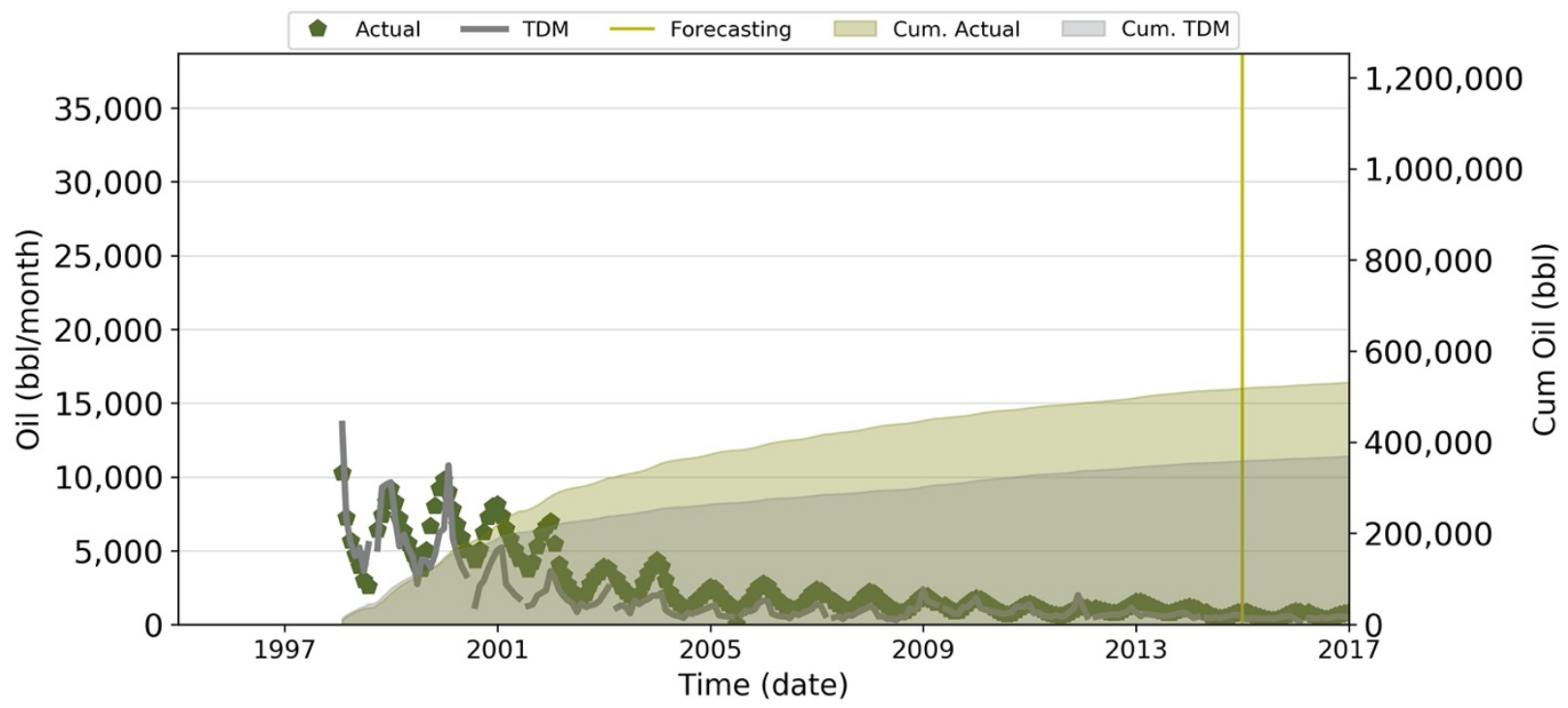

Oil: P-14

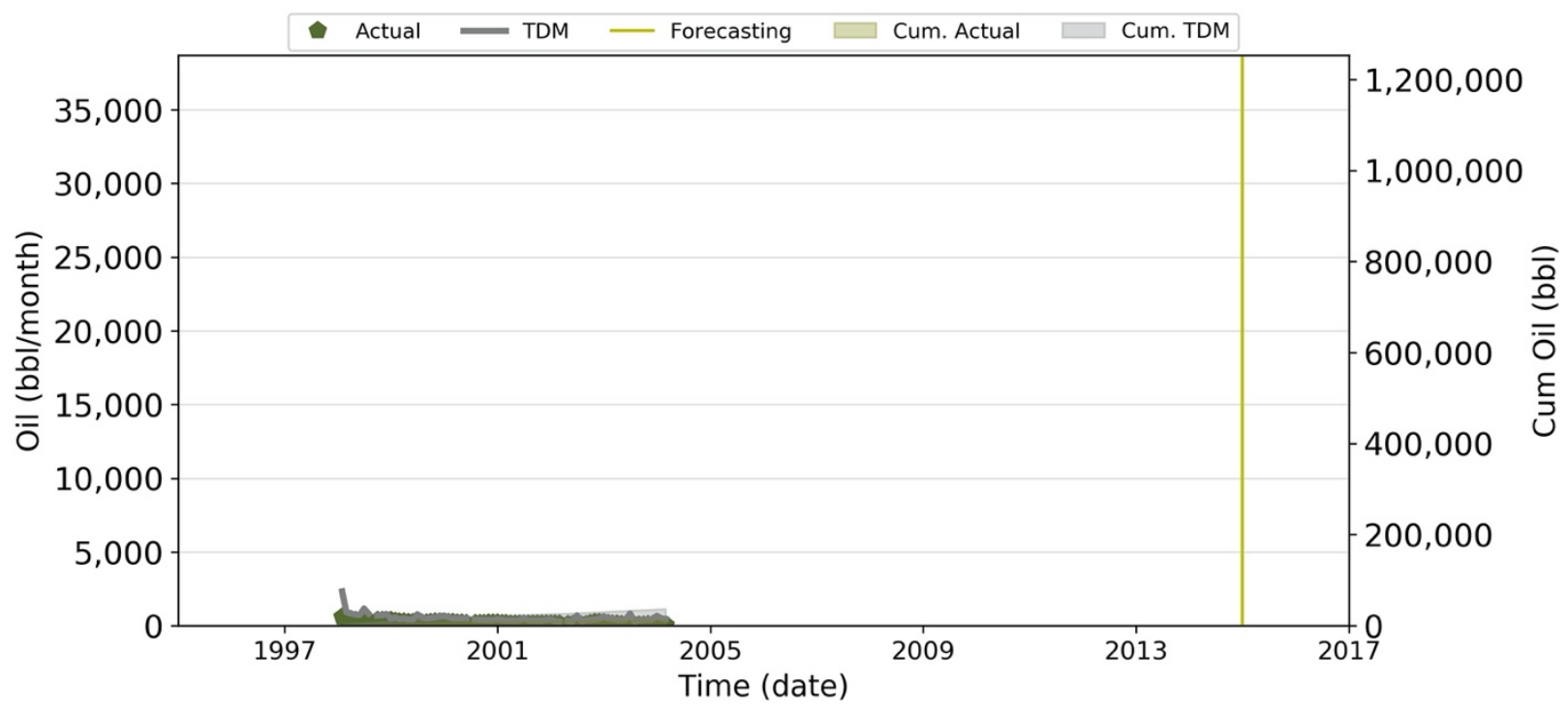


Oil: P-15

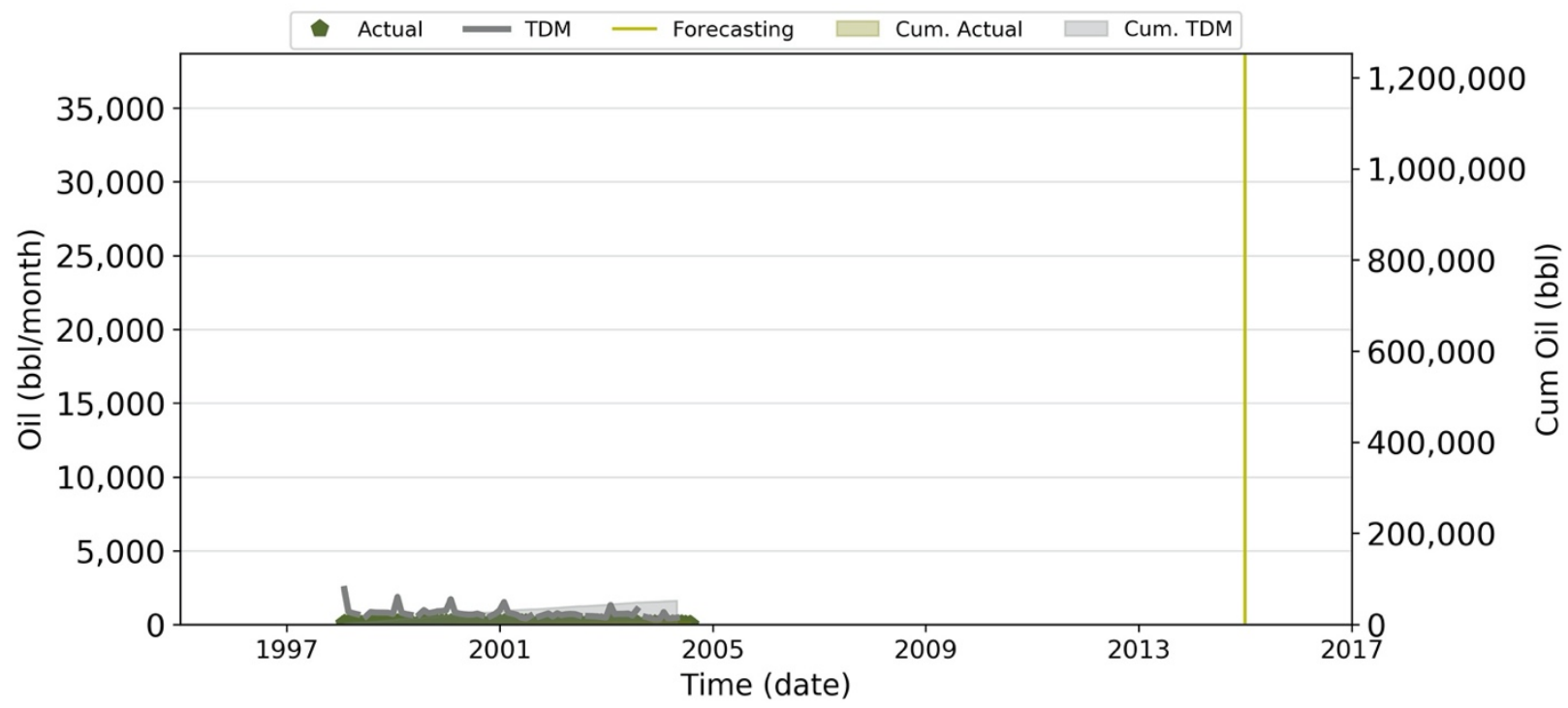

Oil: P-16

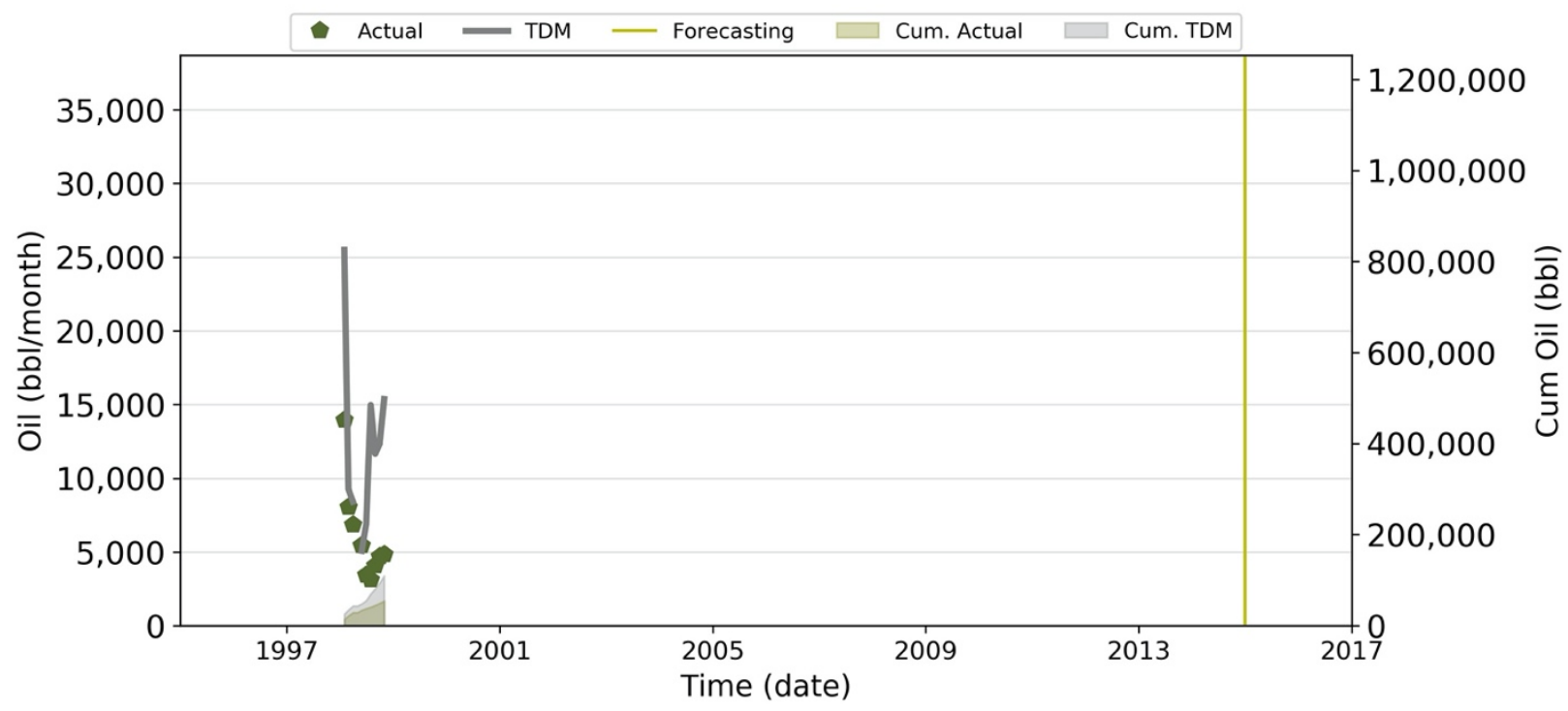


Oil: P-17

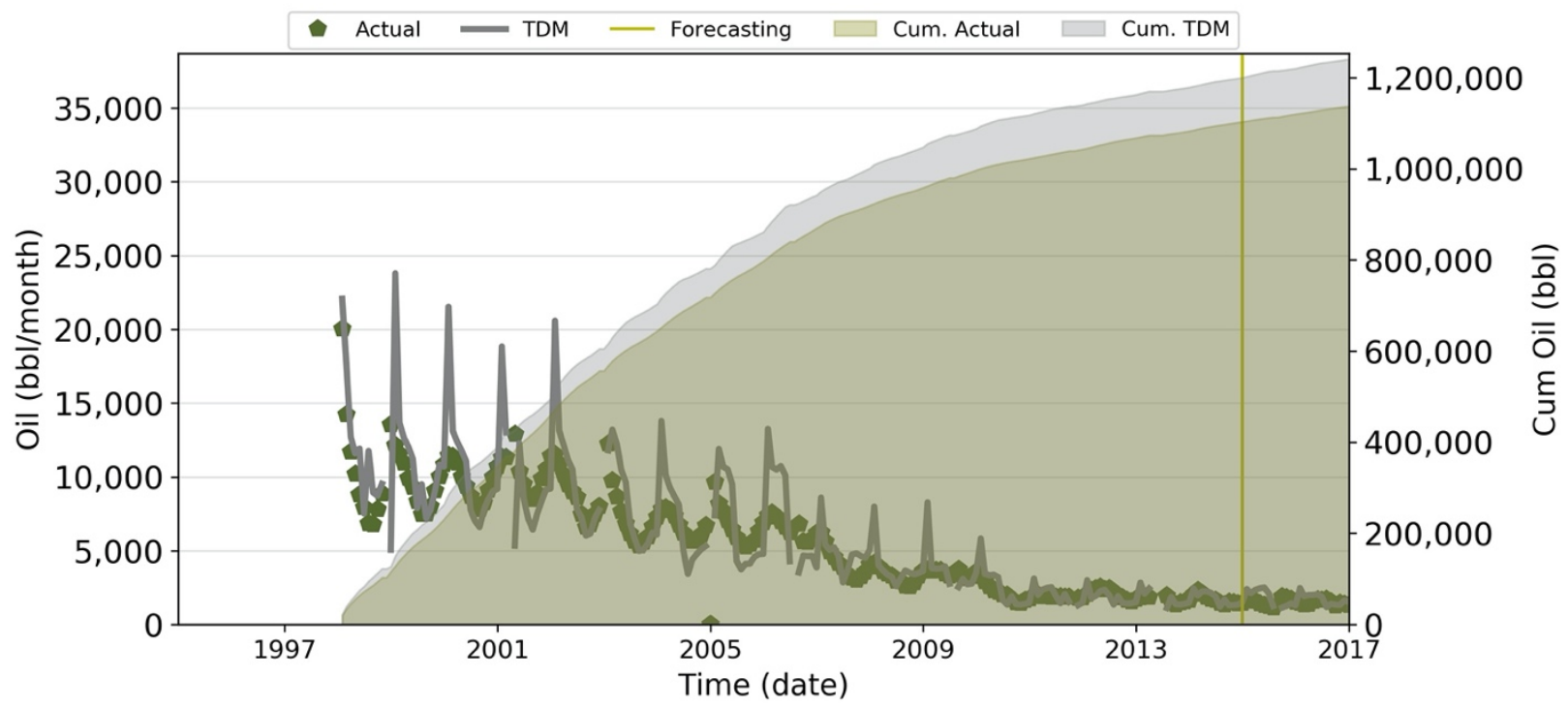

Oil: P-18

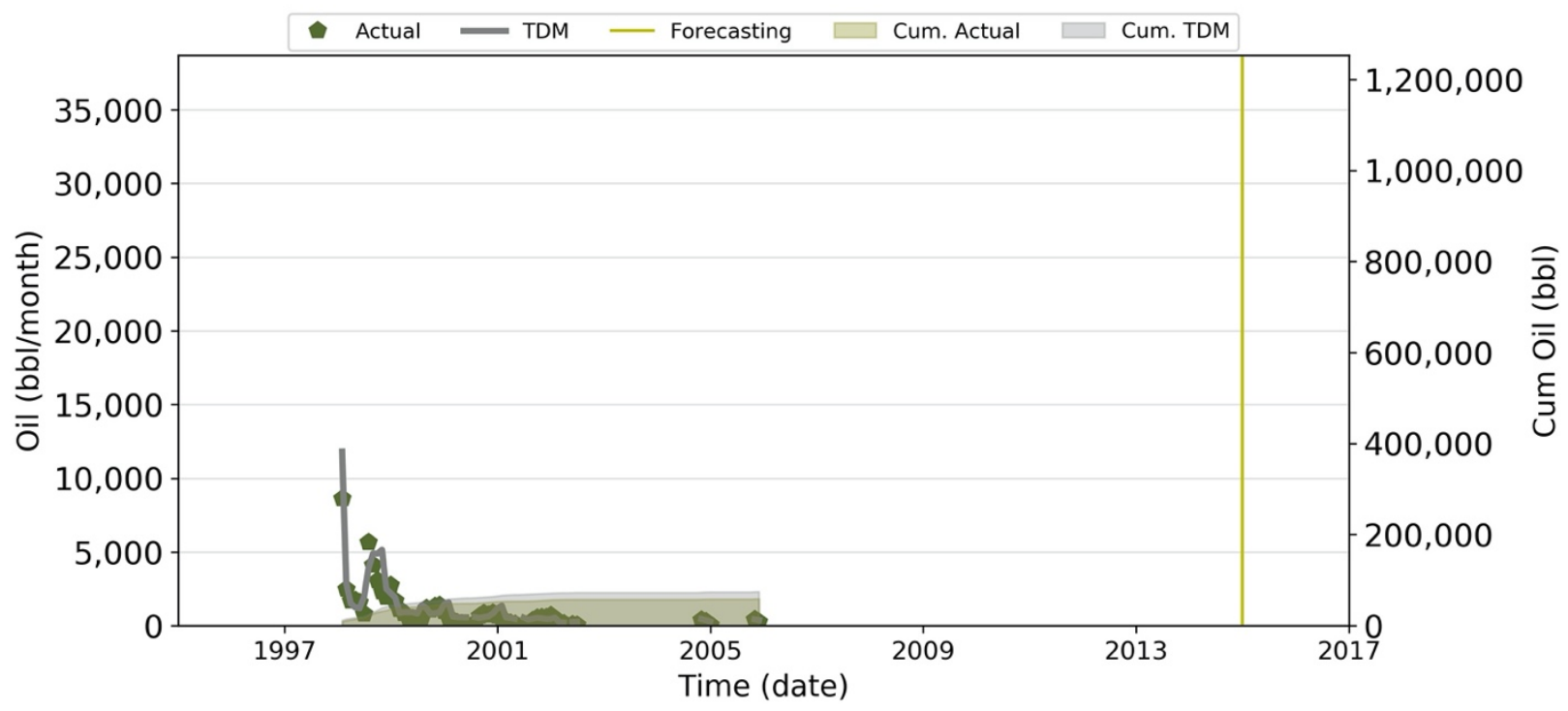


Oil: P-19

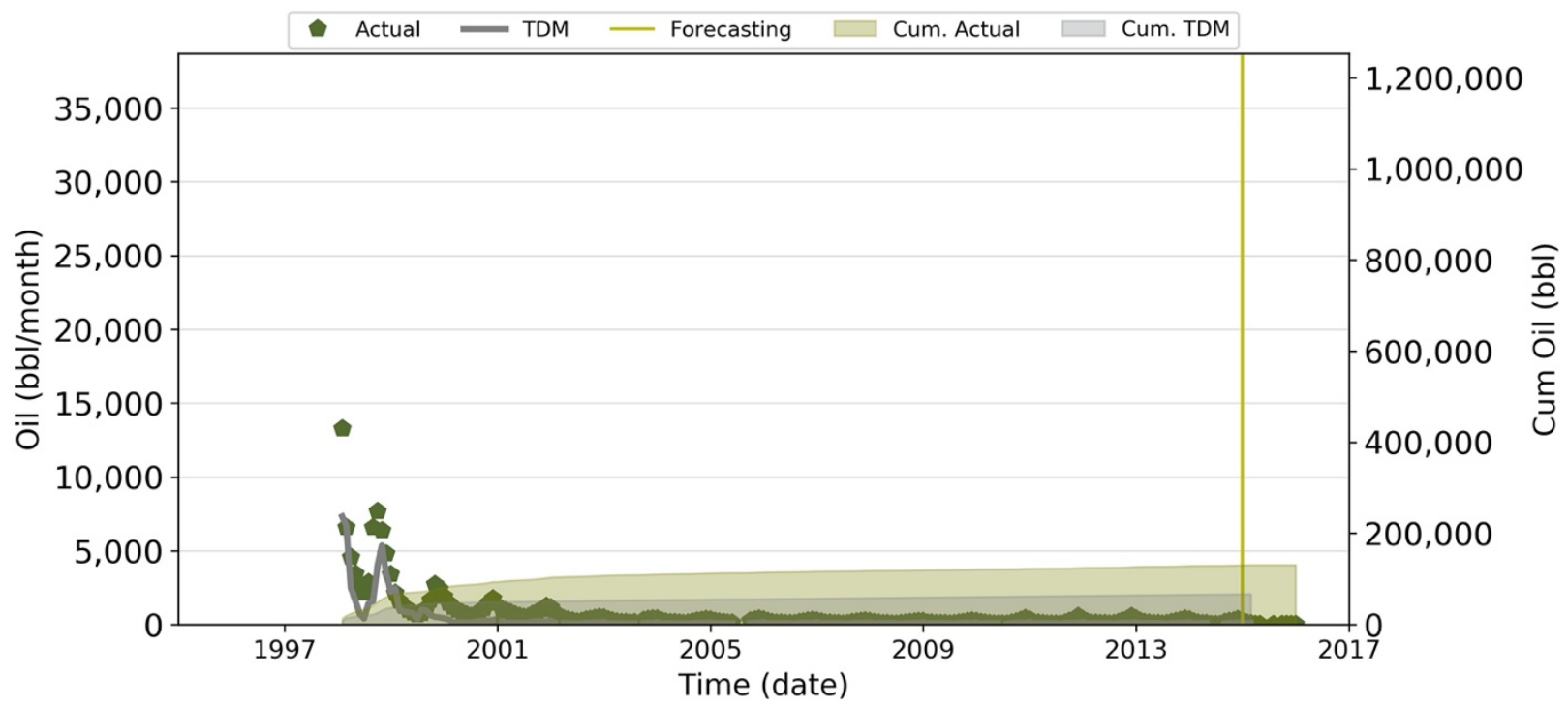

Oil: P-20

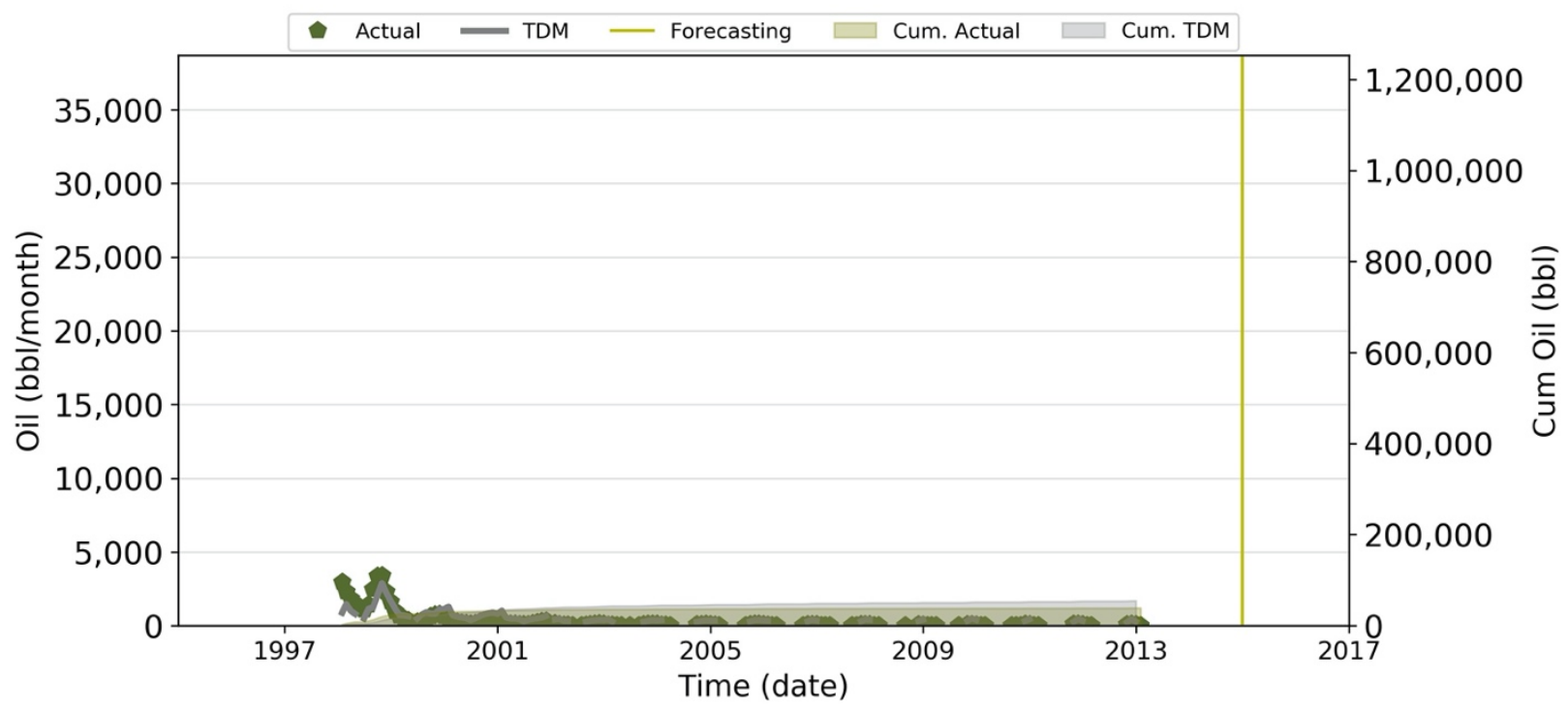


Oil: P-21

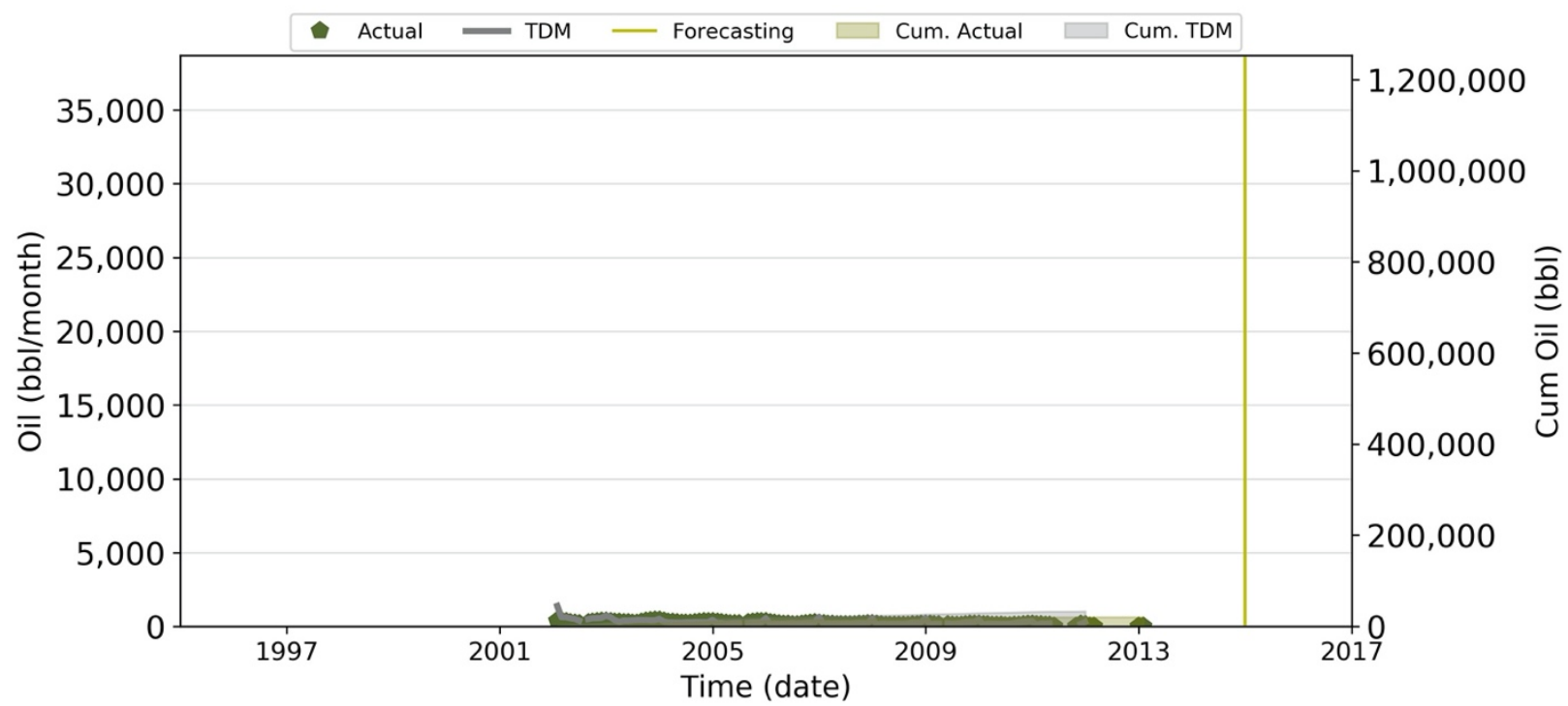

Oil: P-22

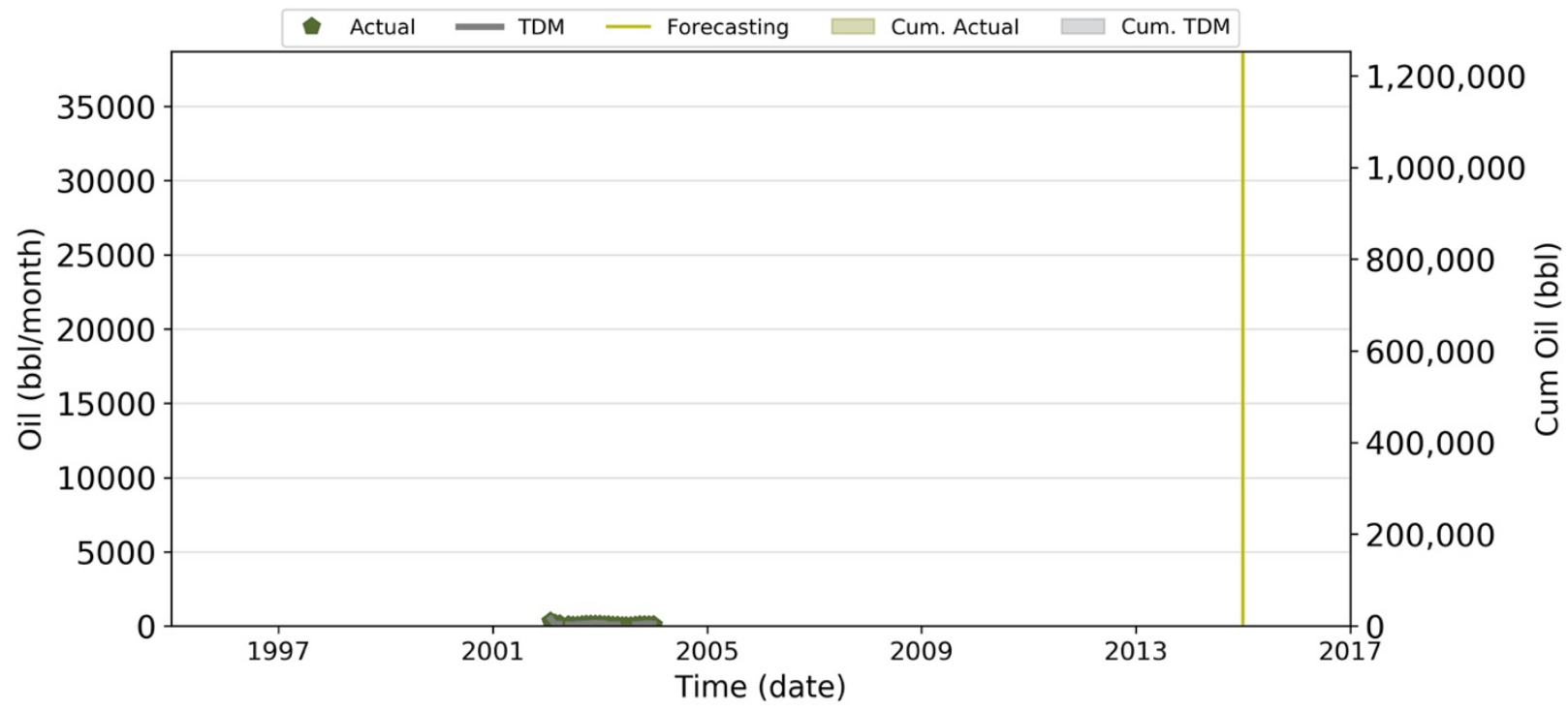


Oil: P-23

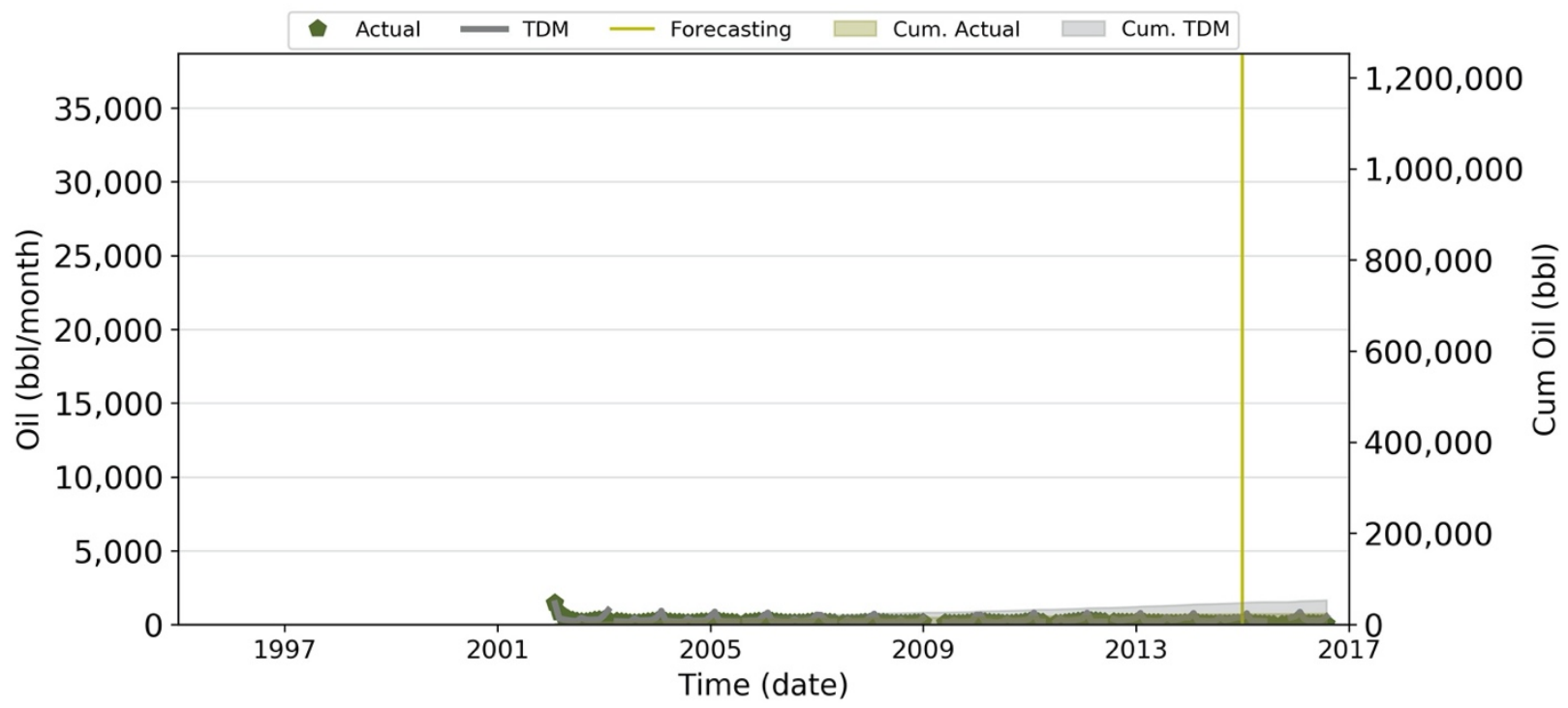

Oil: P-24

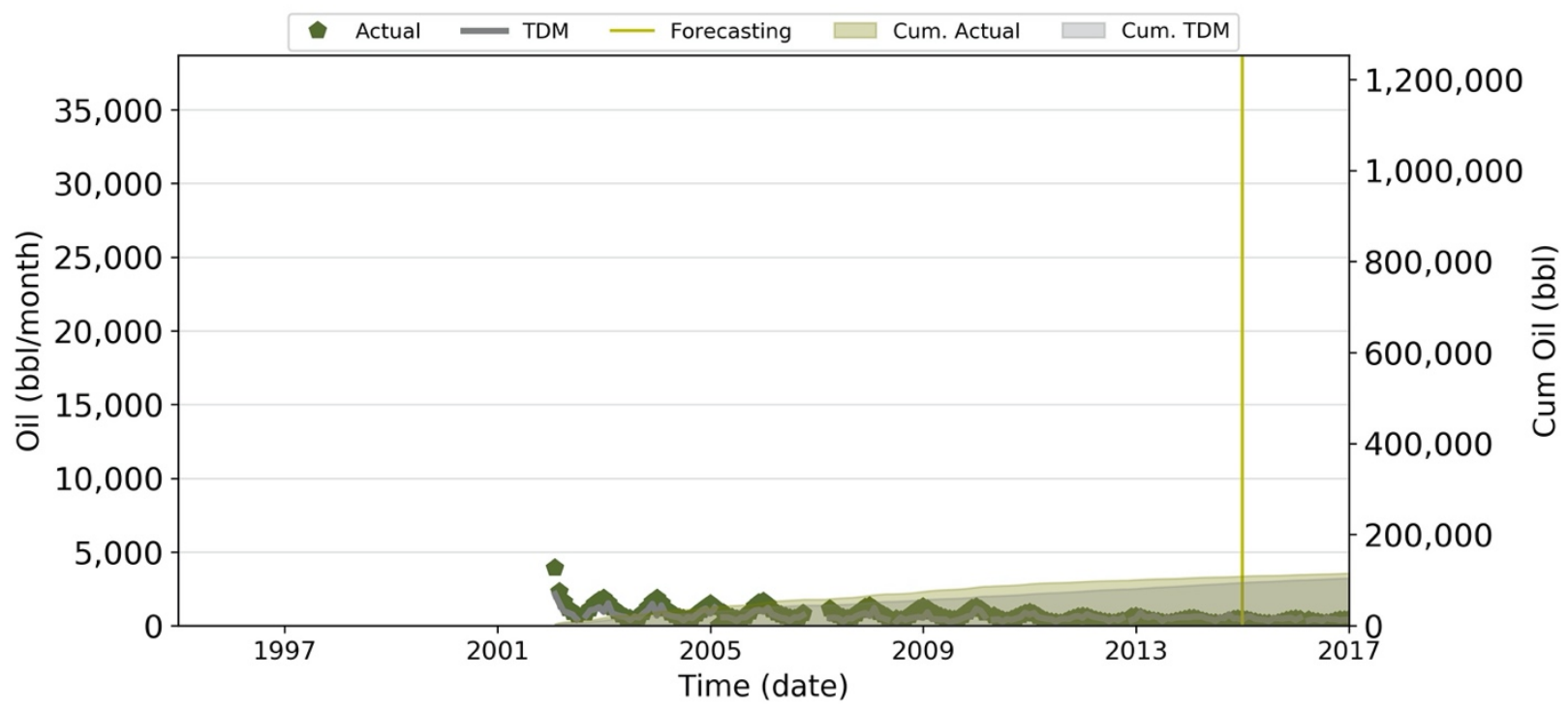


Oil: P-25

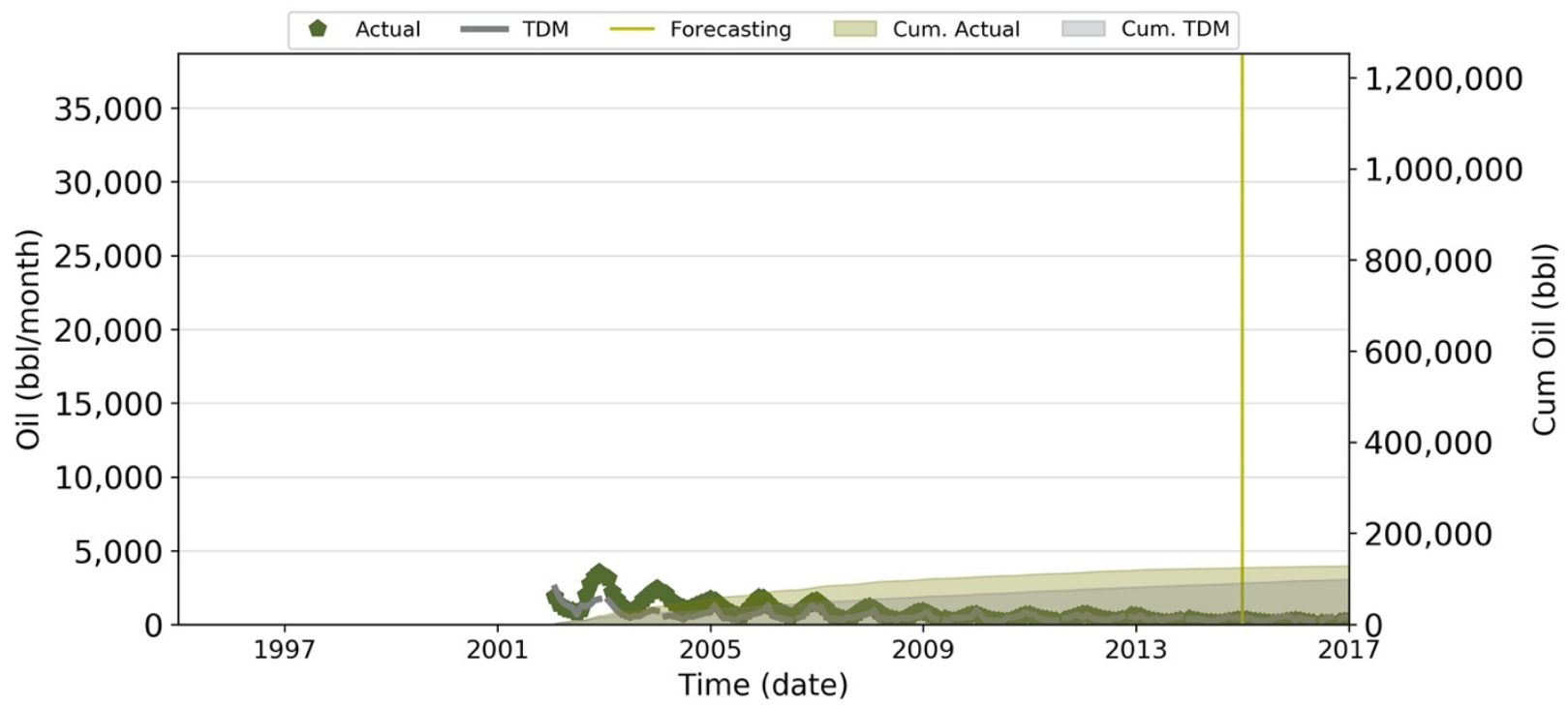

Oil: P-26

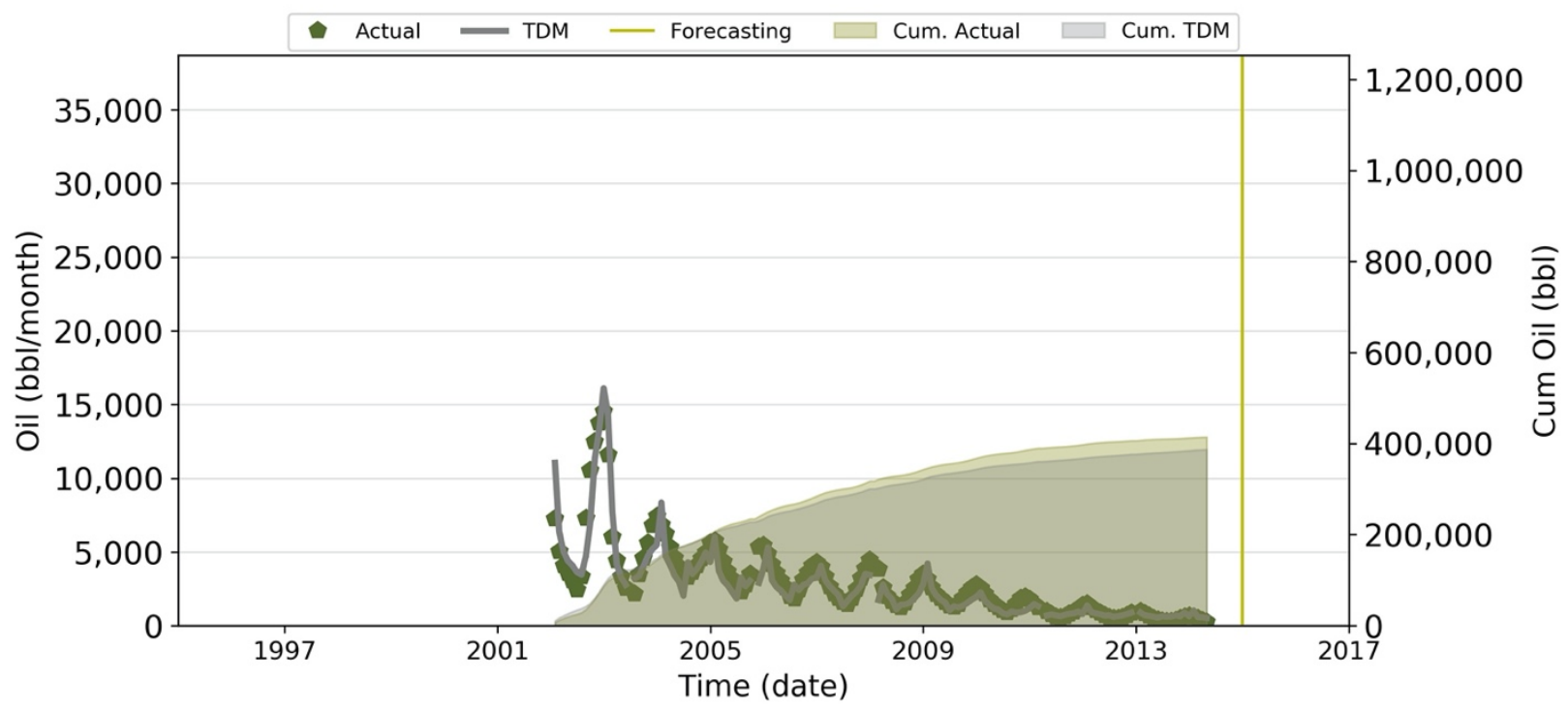


Oil: P-27

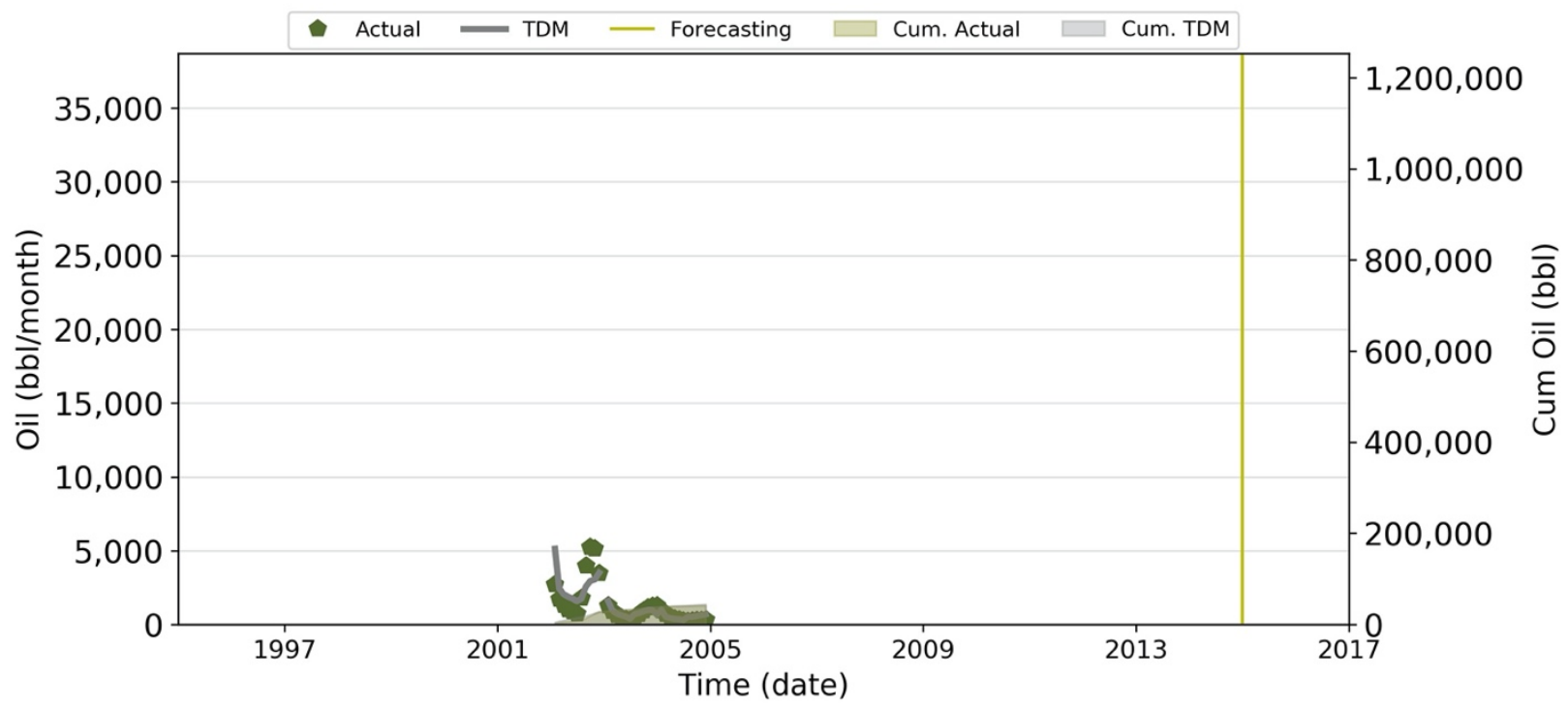

Oil: P-28

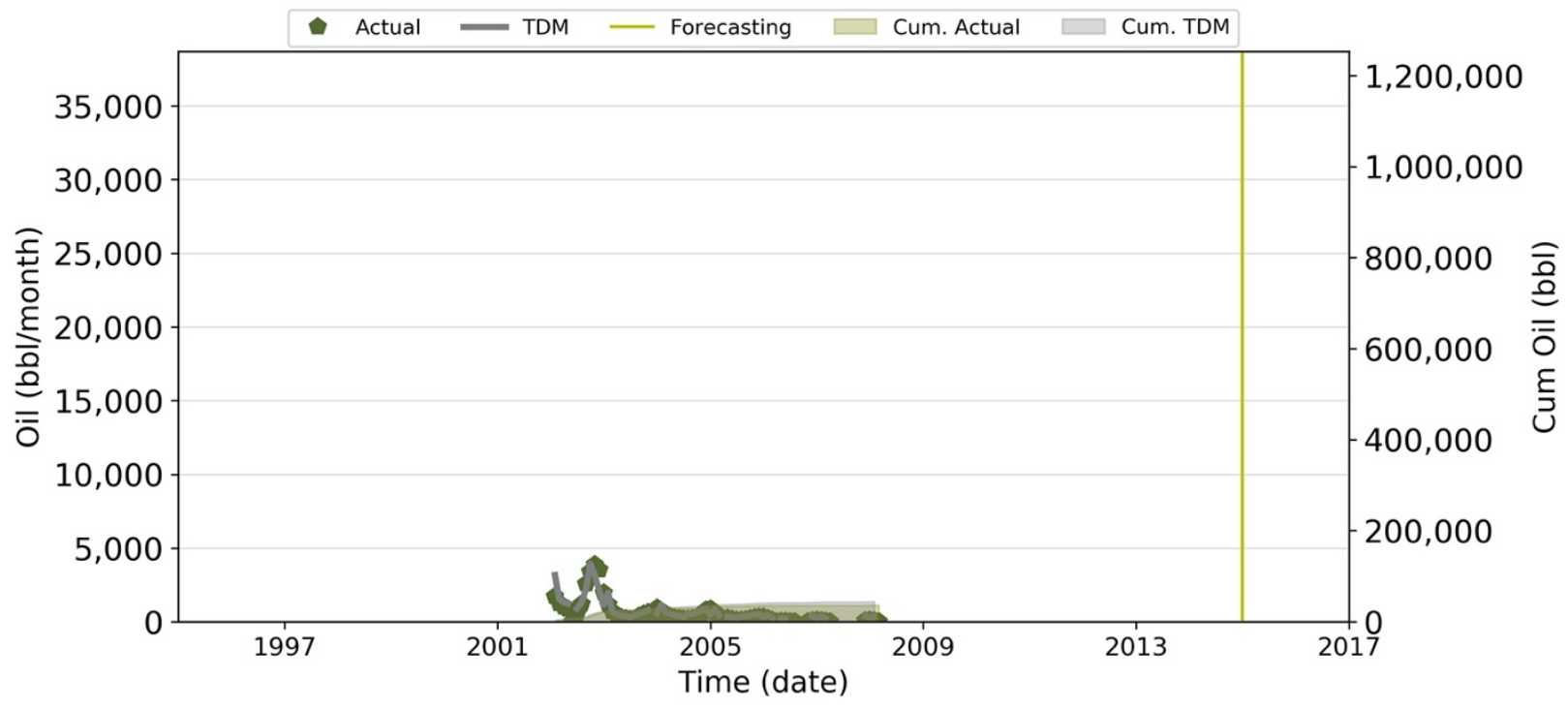


Oil: P-29

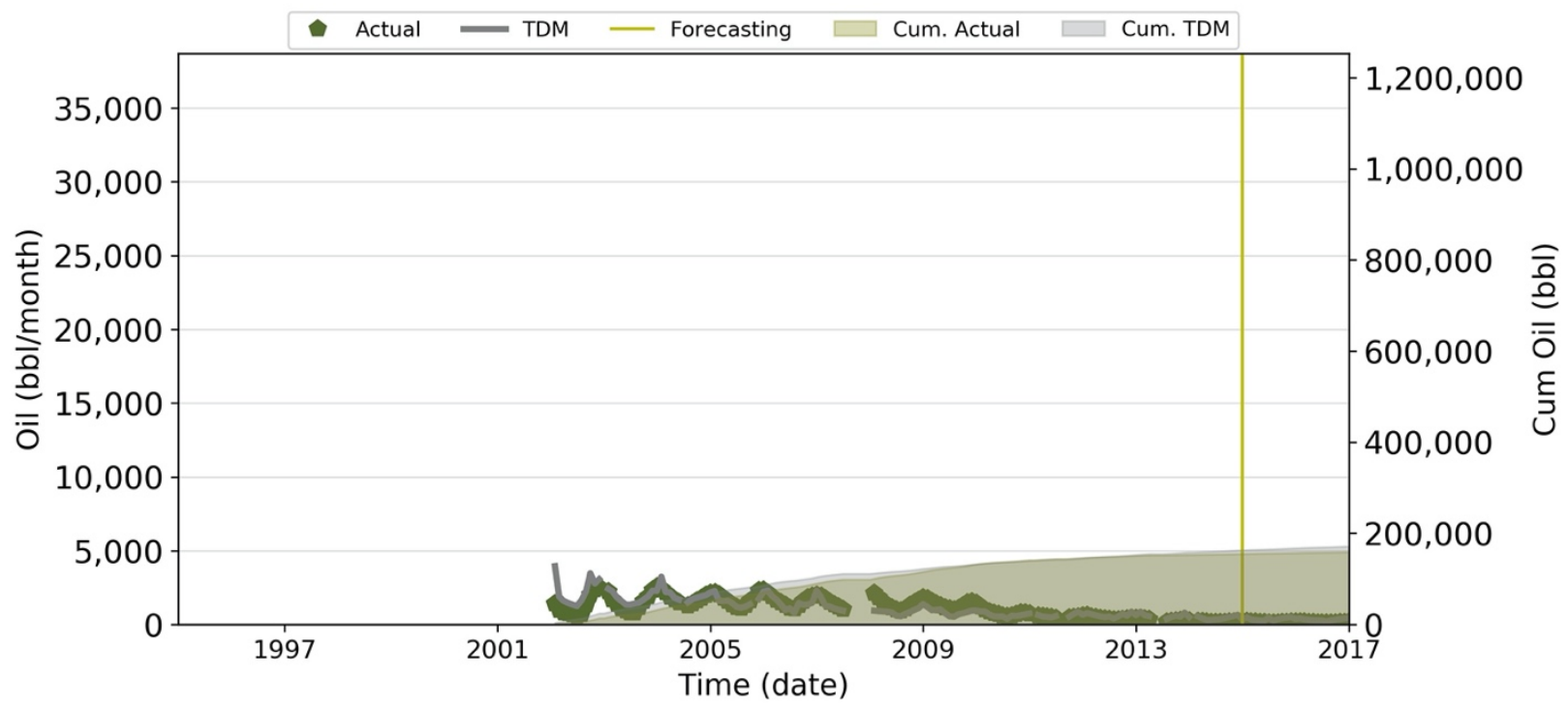

Oil: P-30

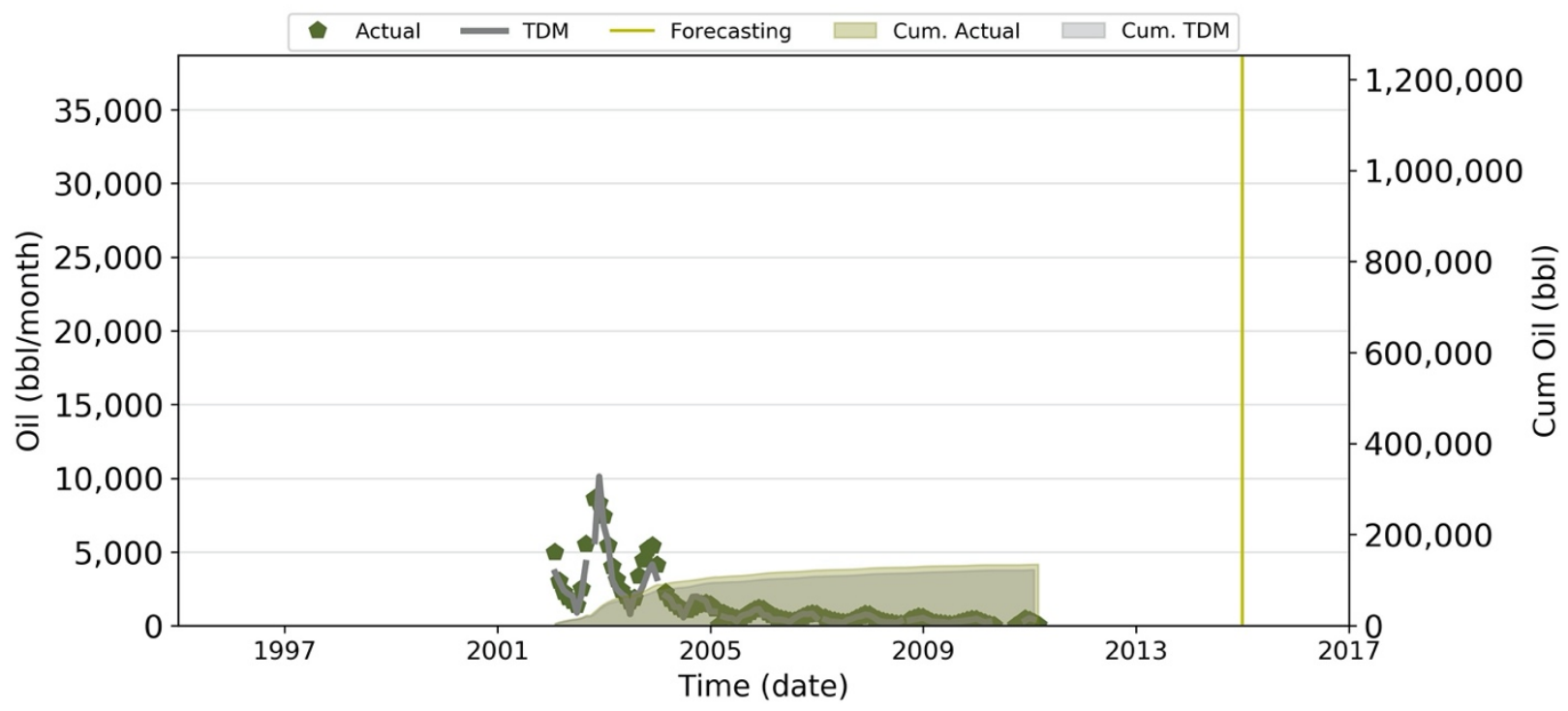


Oil: P-31

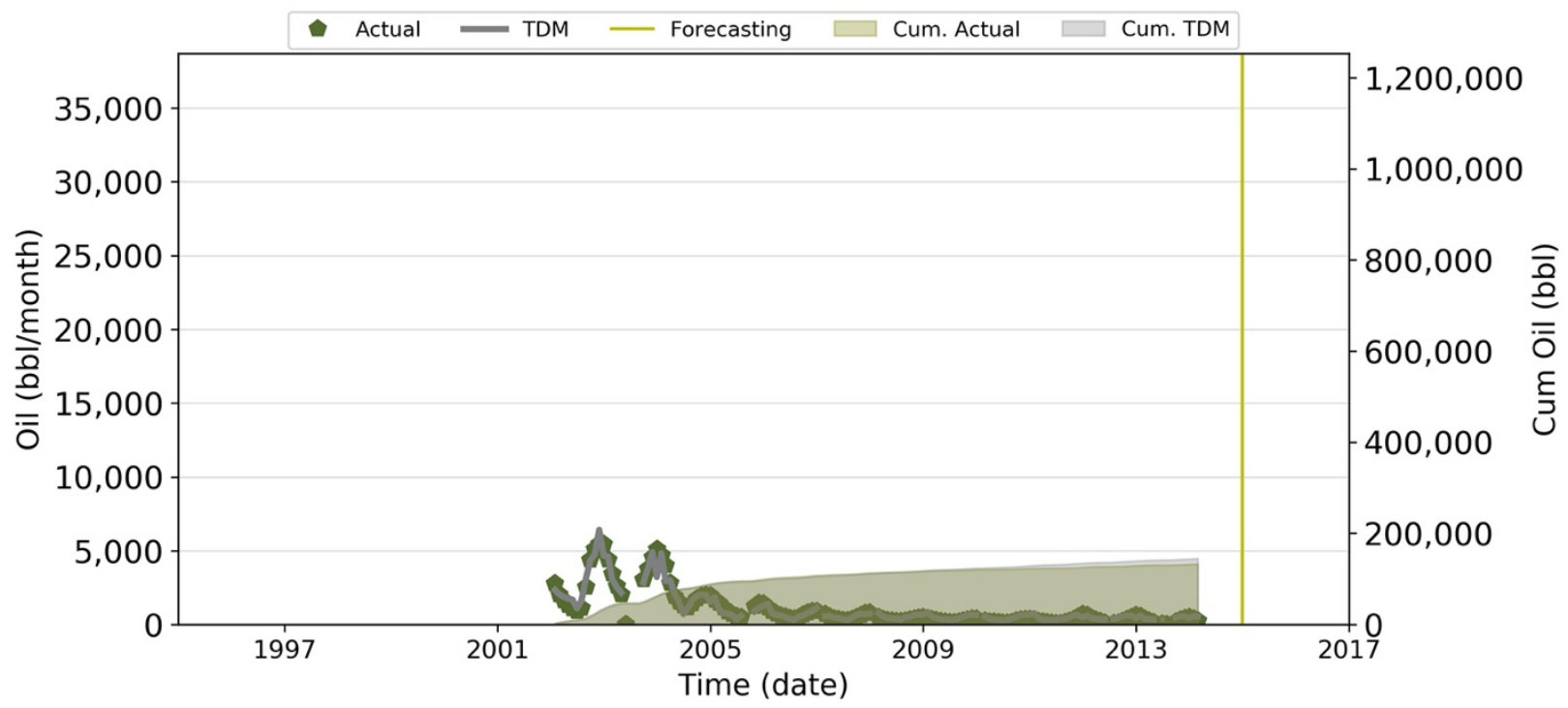

Oil: P-32

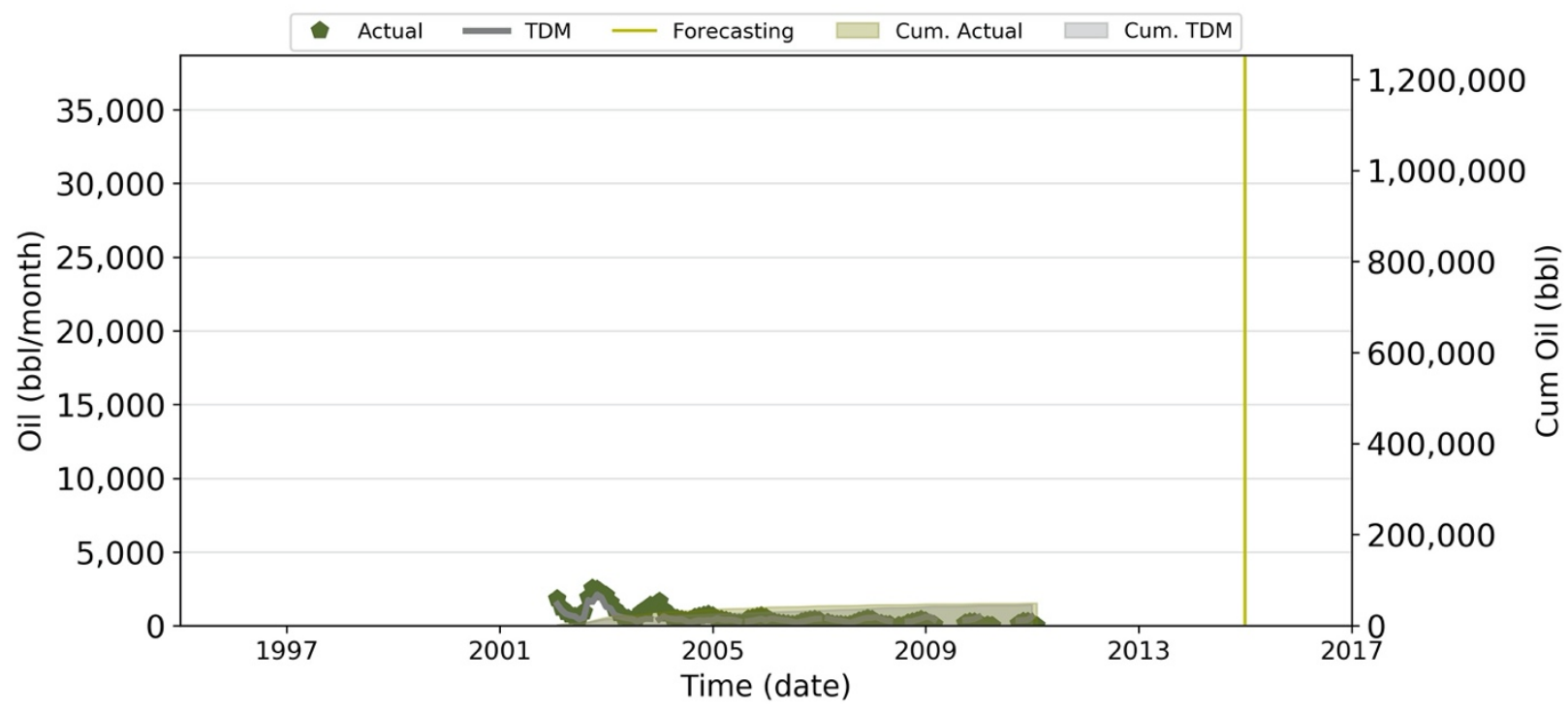


Oil: P-33

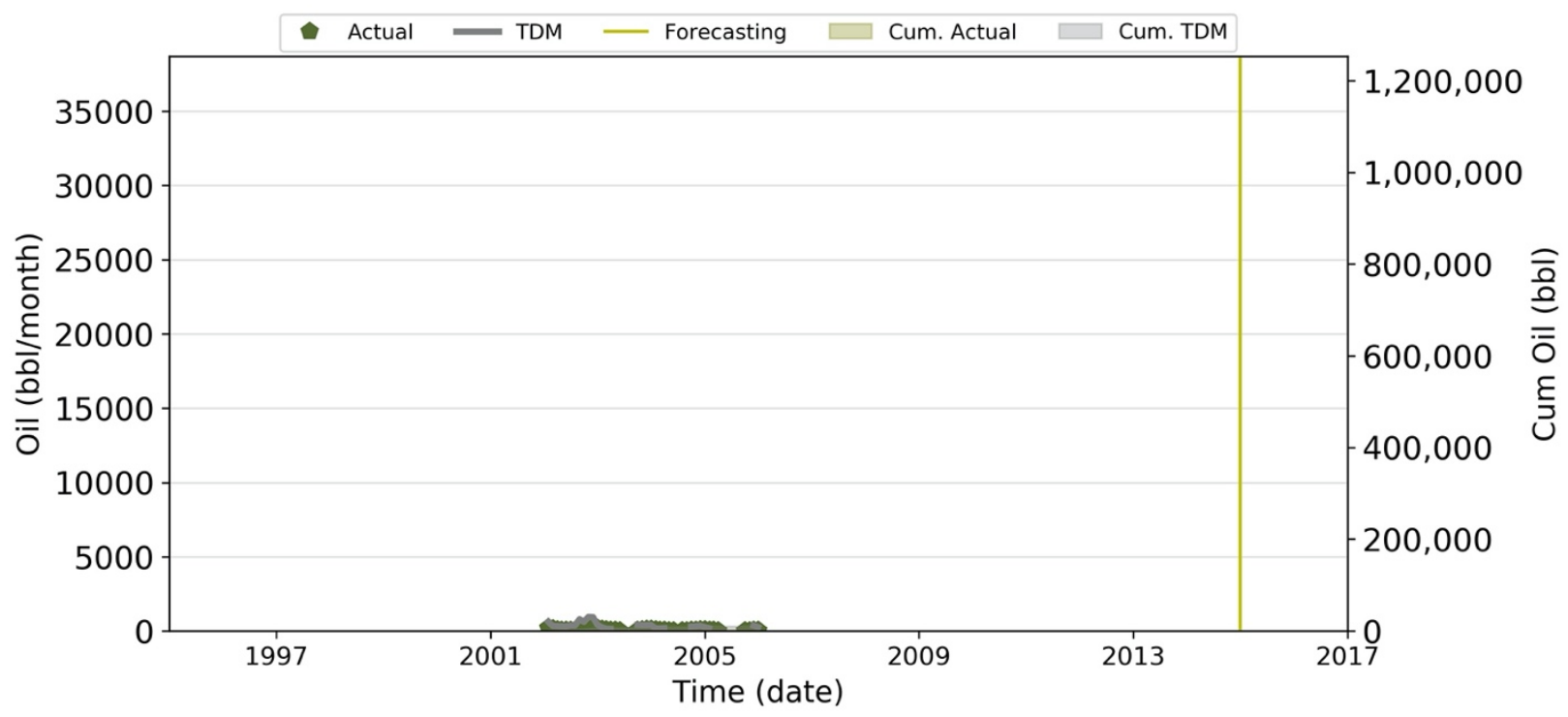

Oil: P-34

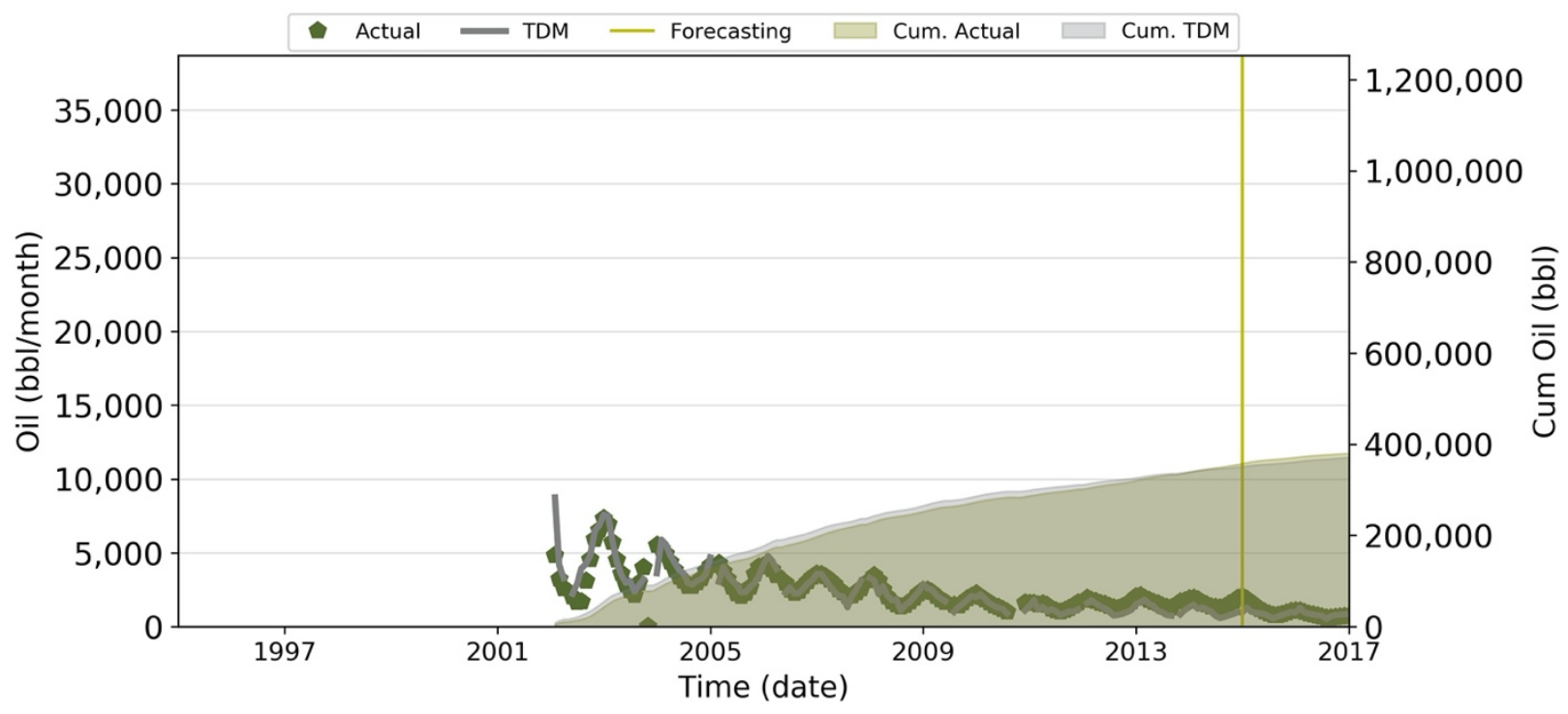


Oil: P-37

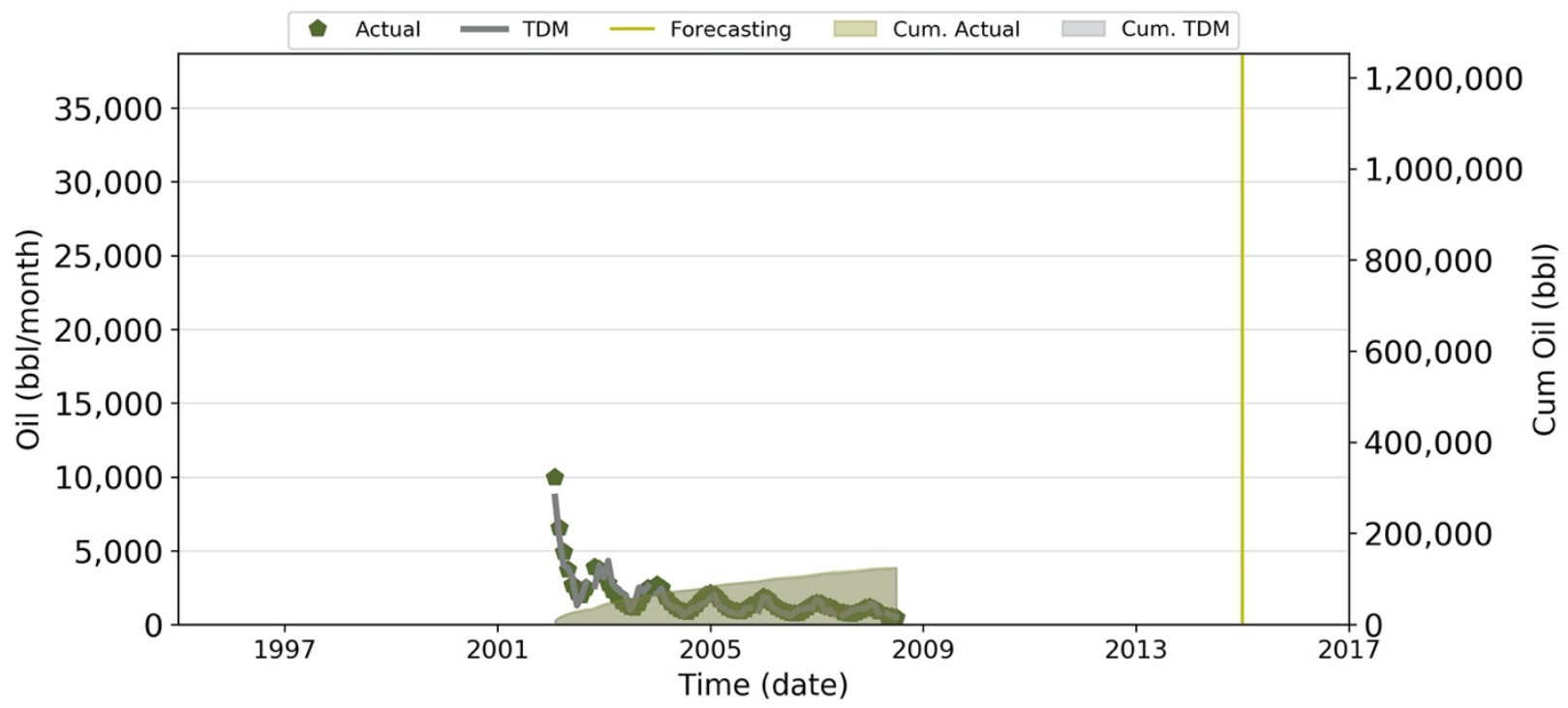

Oil: P-38

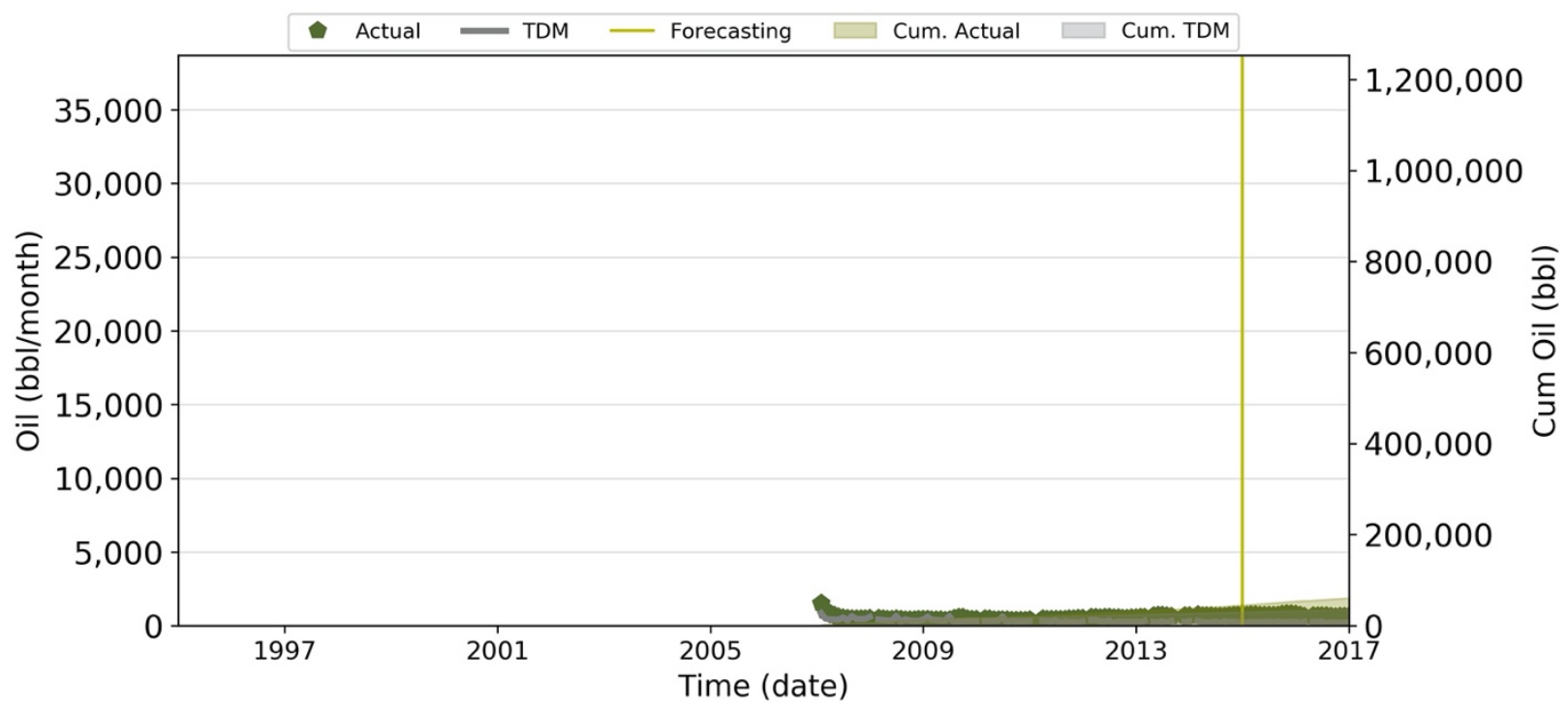


Oil: P-39

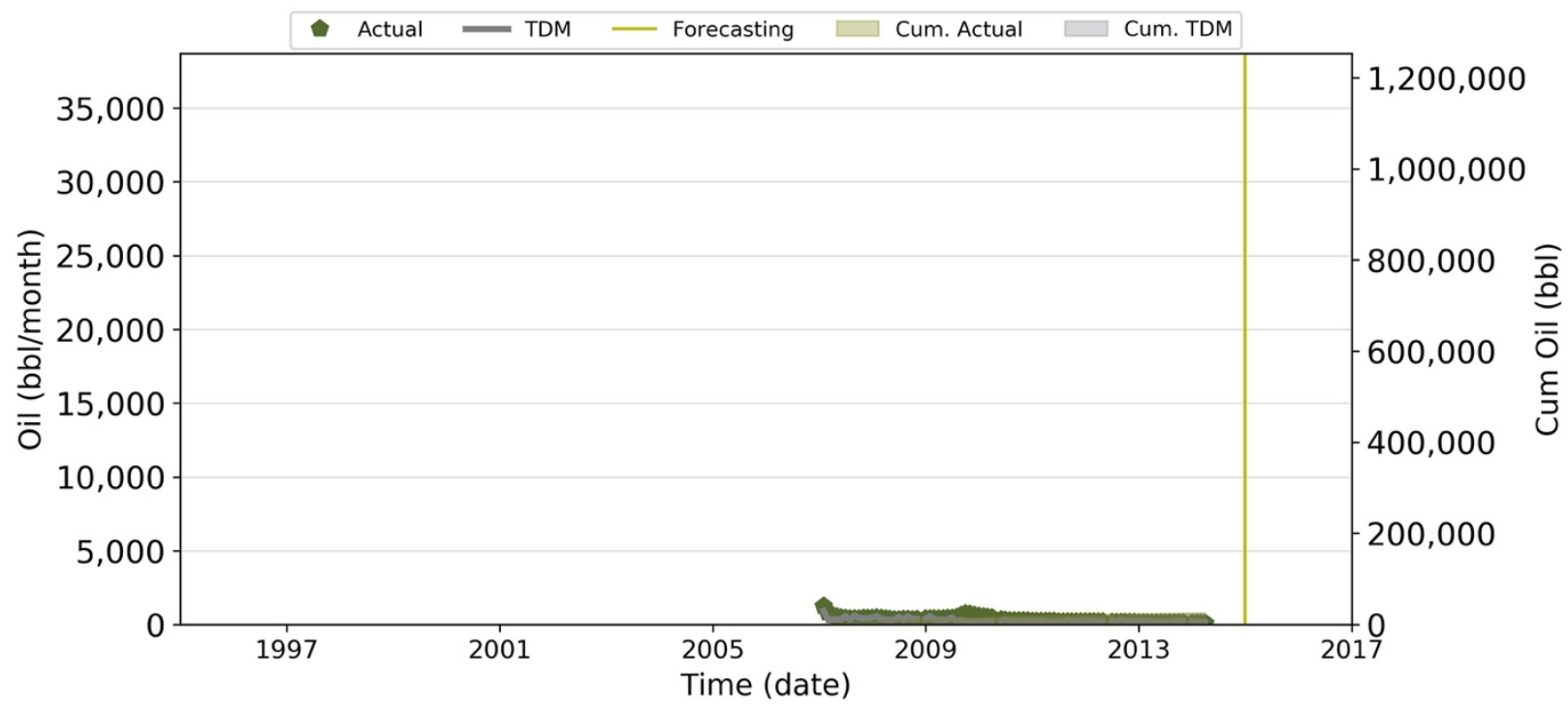

Oil: P-41

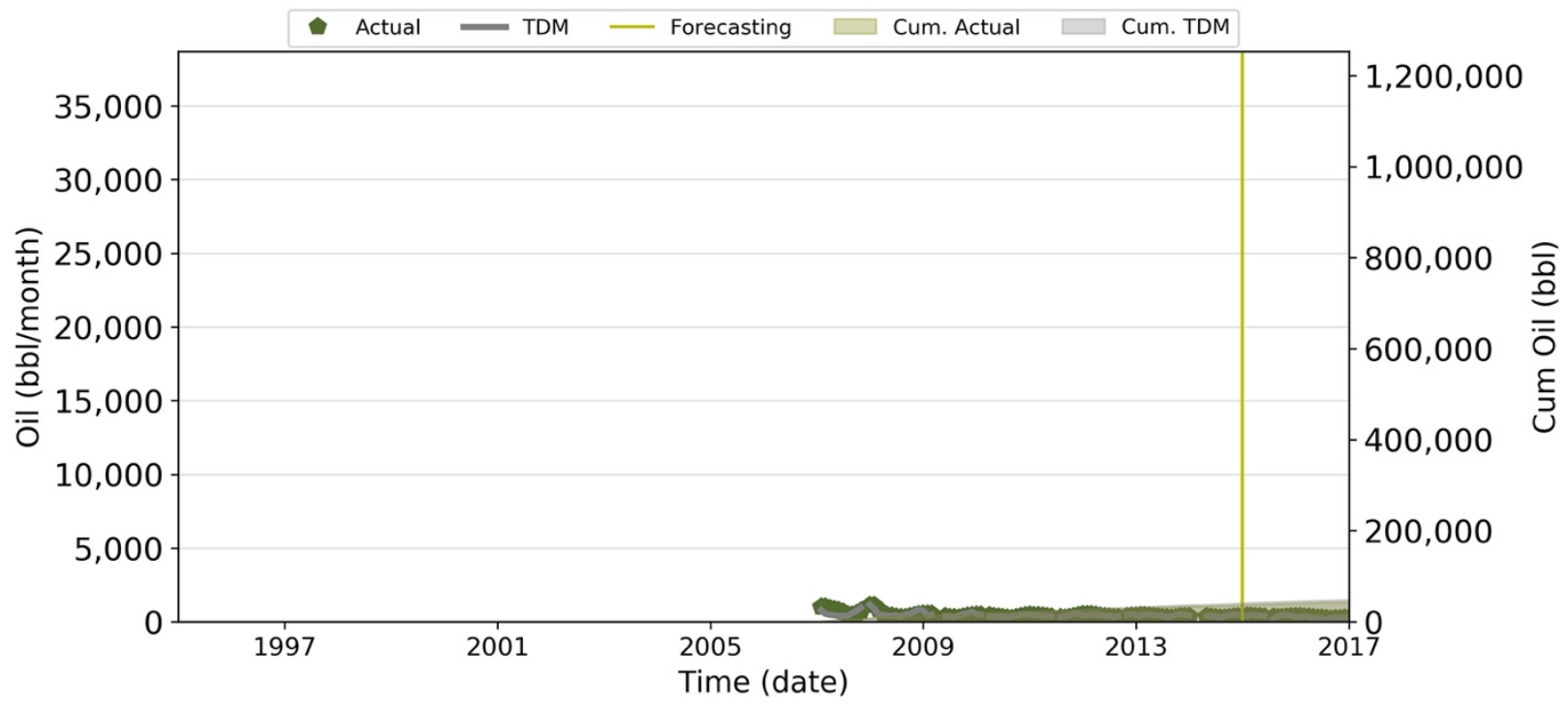


Oil: P-42

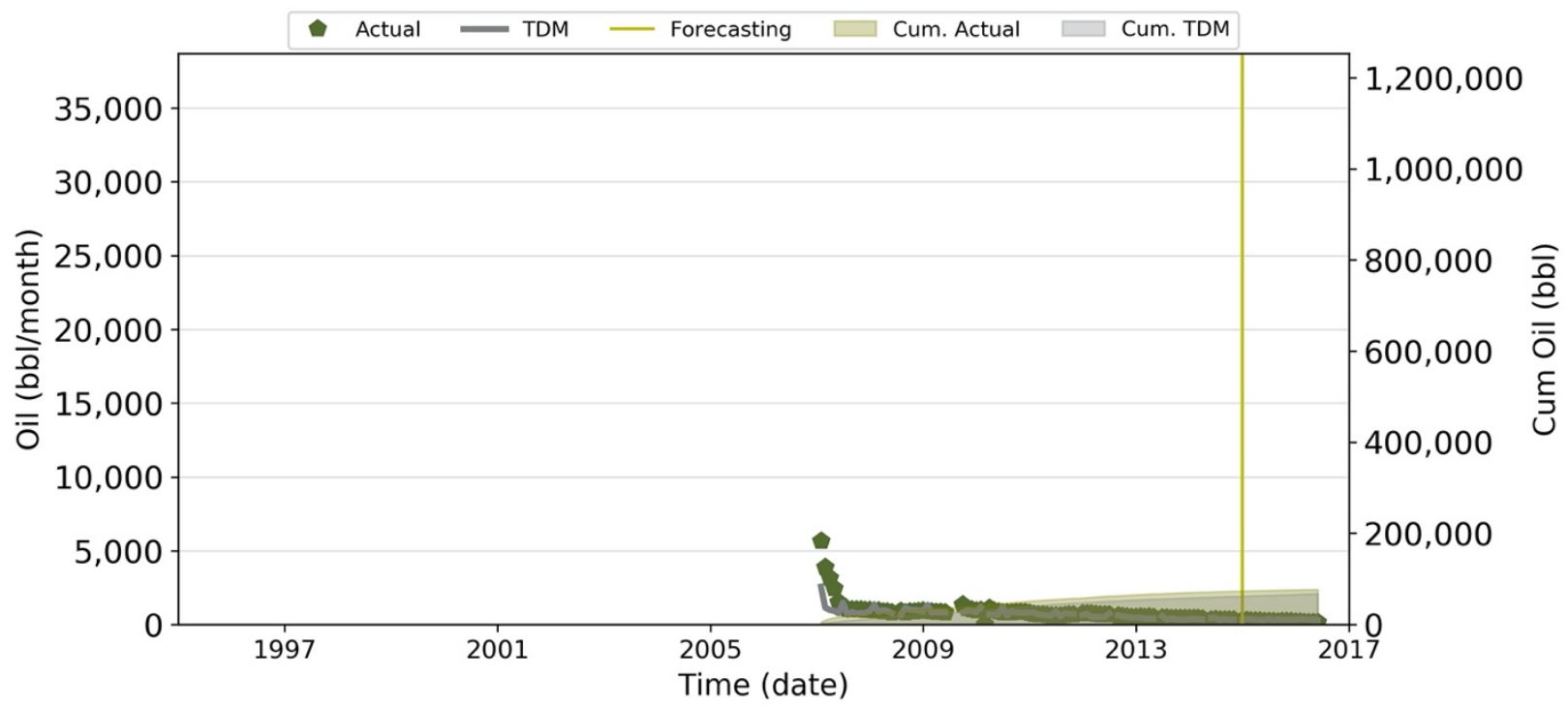

Oil: P-43

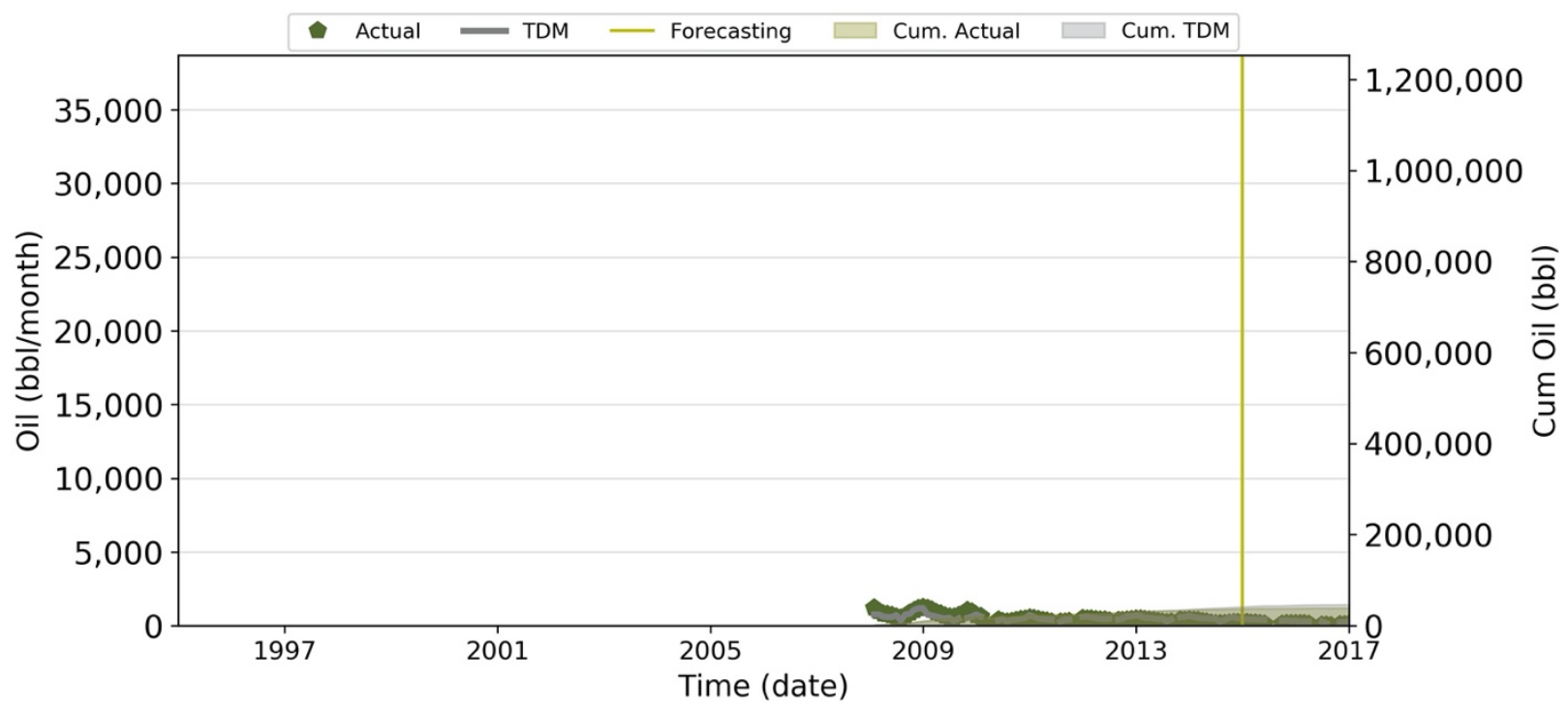


Oil: P-44

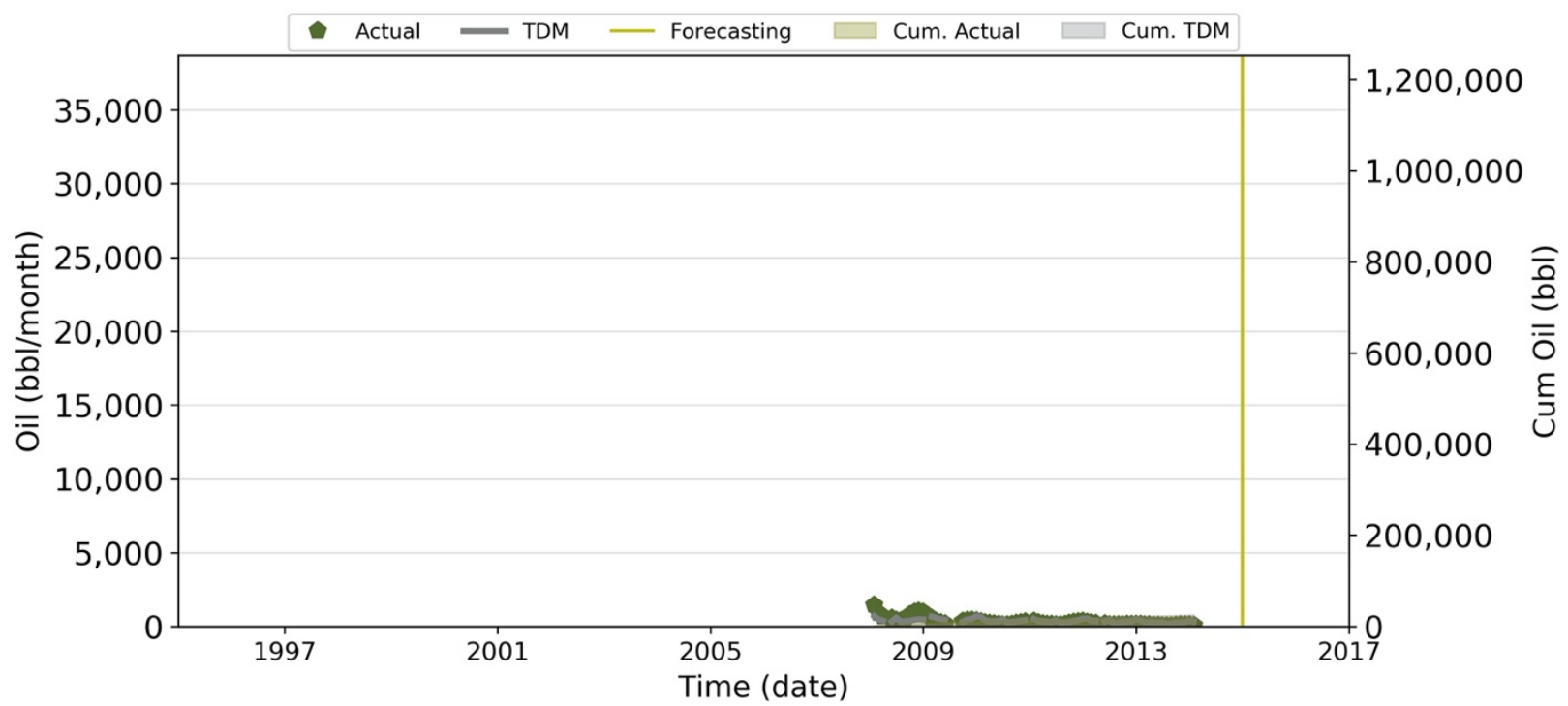

Oil: P-47

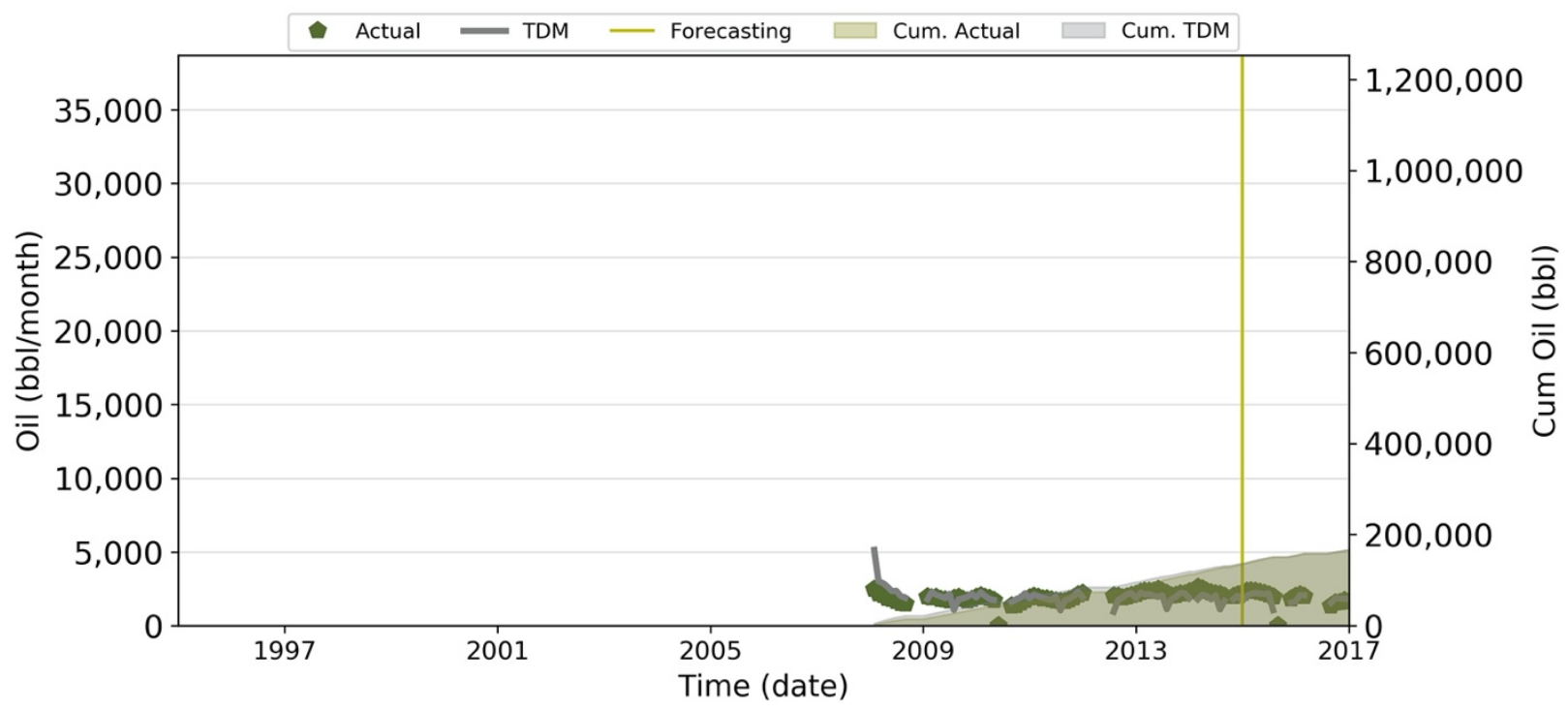


Oil: P-49

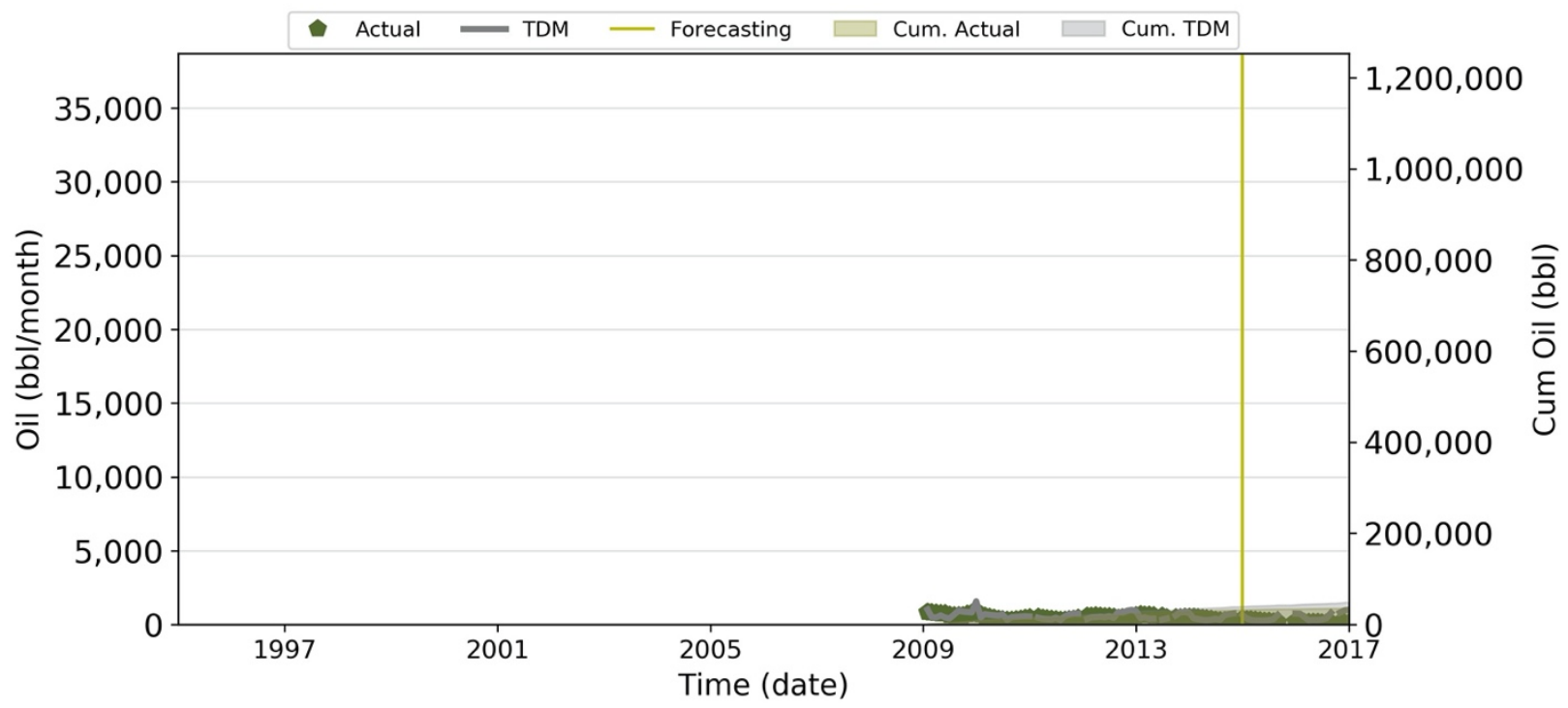

Oil: P-50

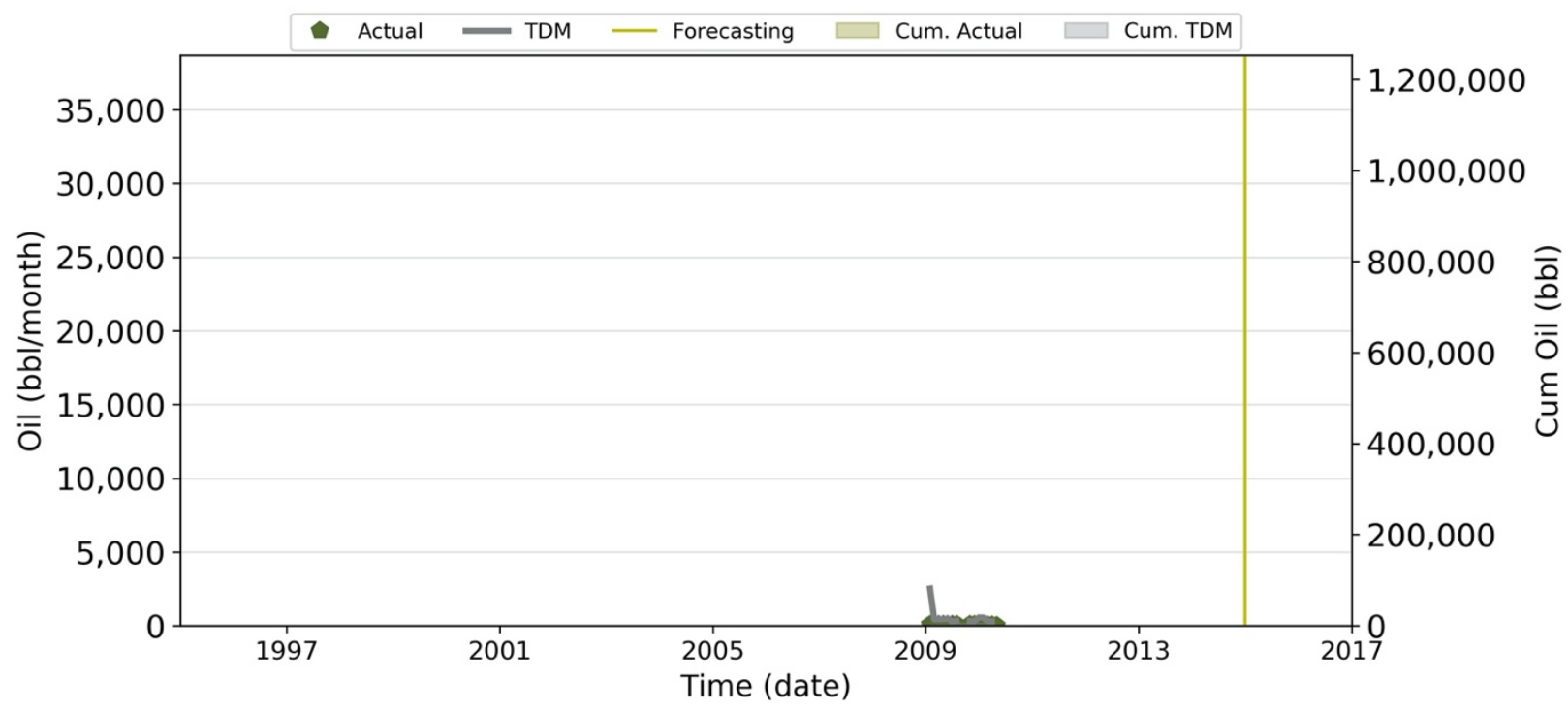


Oil: P-51

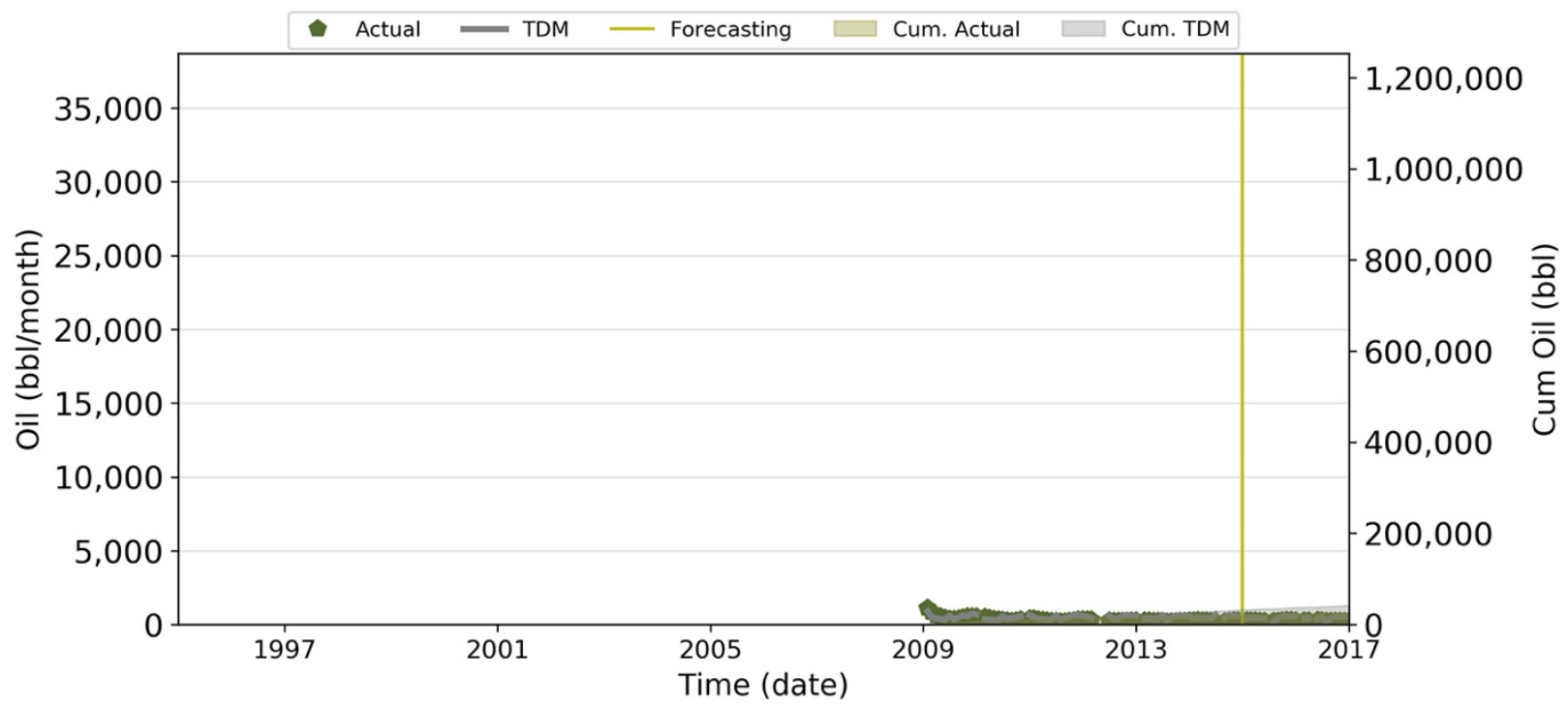

Oil: P-52

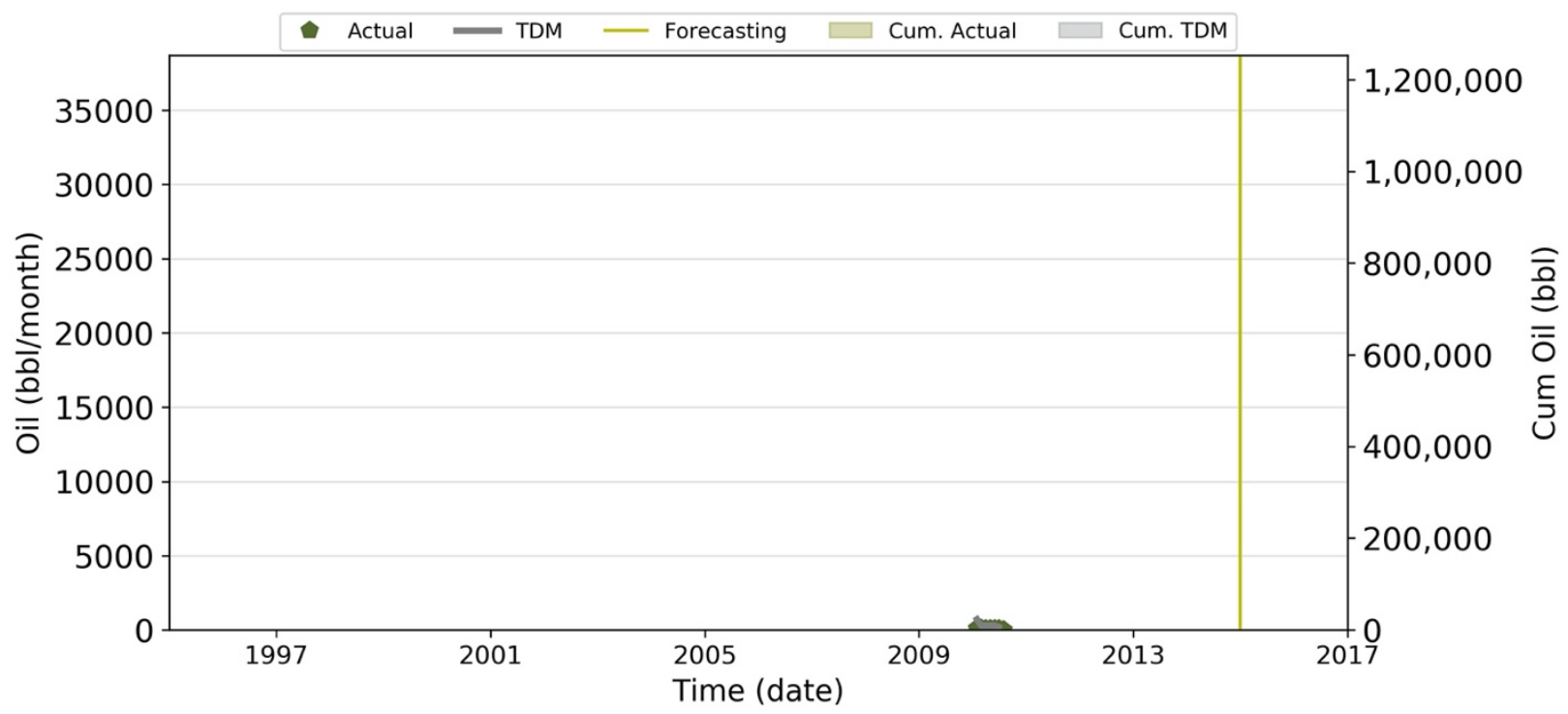


Oil: P-53

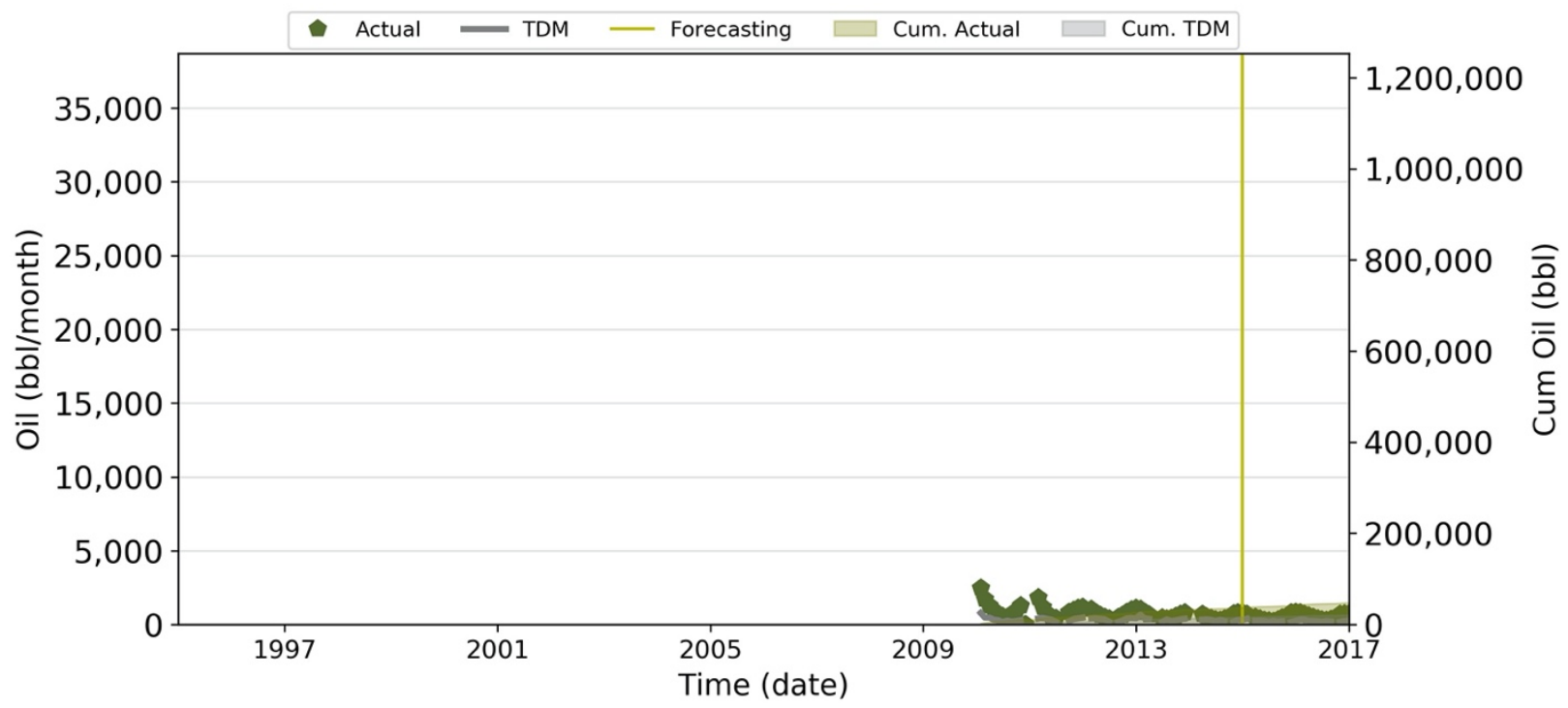

Oil: P-54

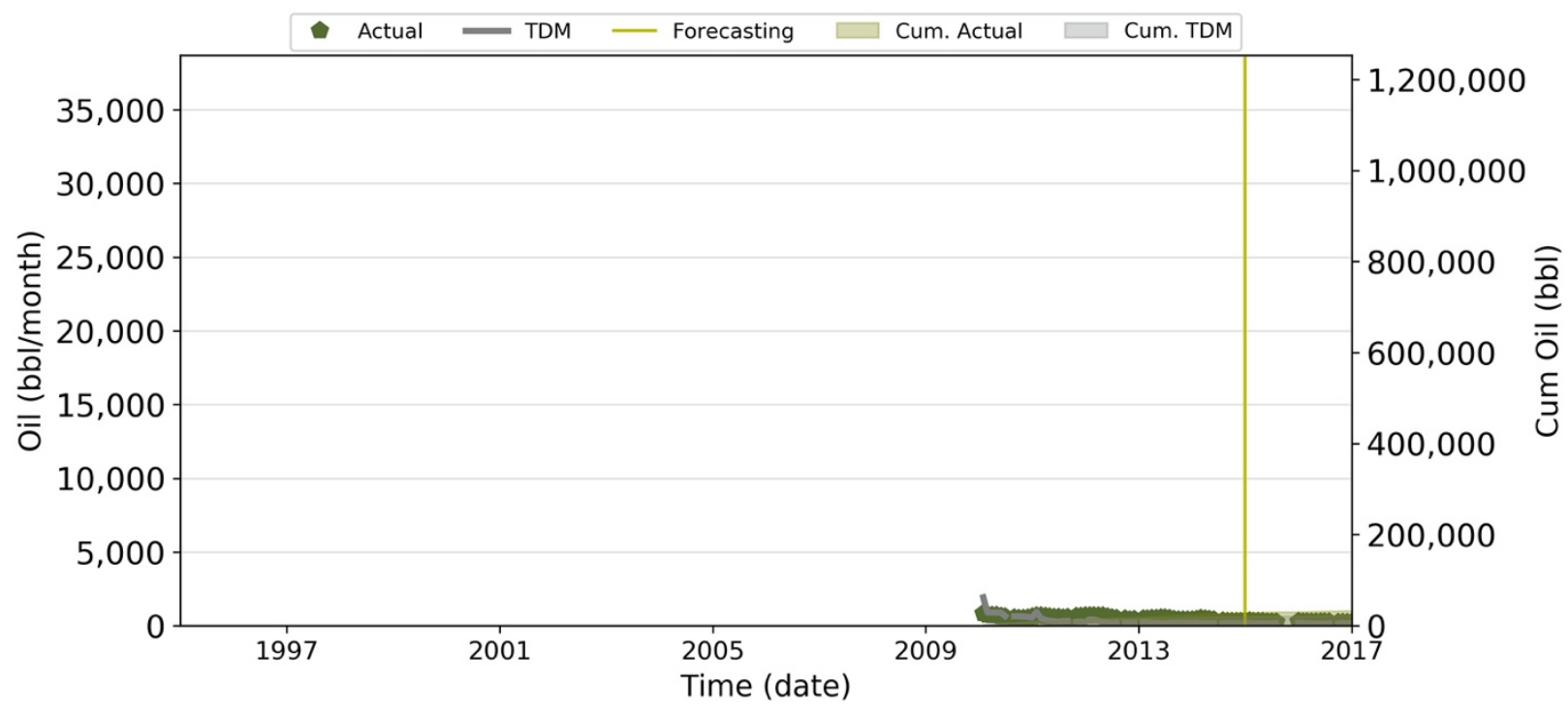


Oil: P-55

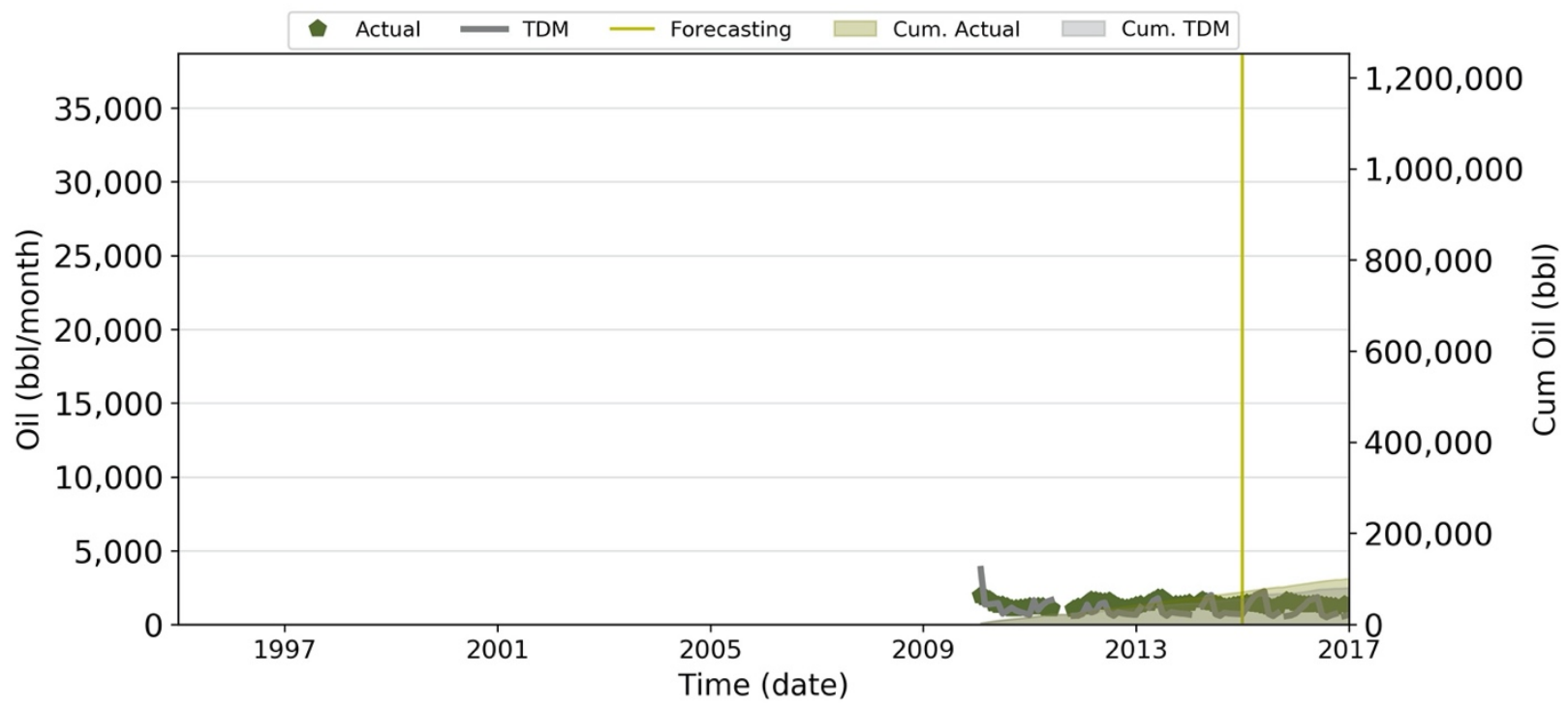

Oil: P-56

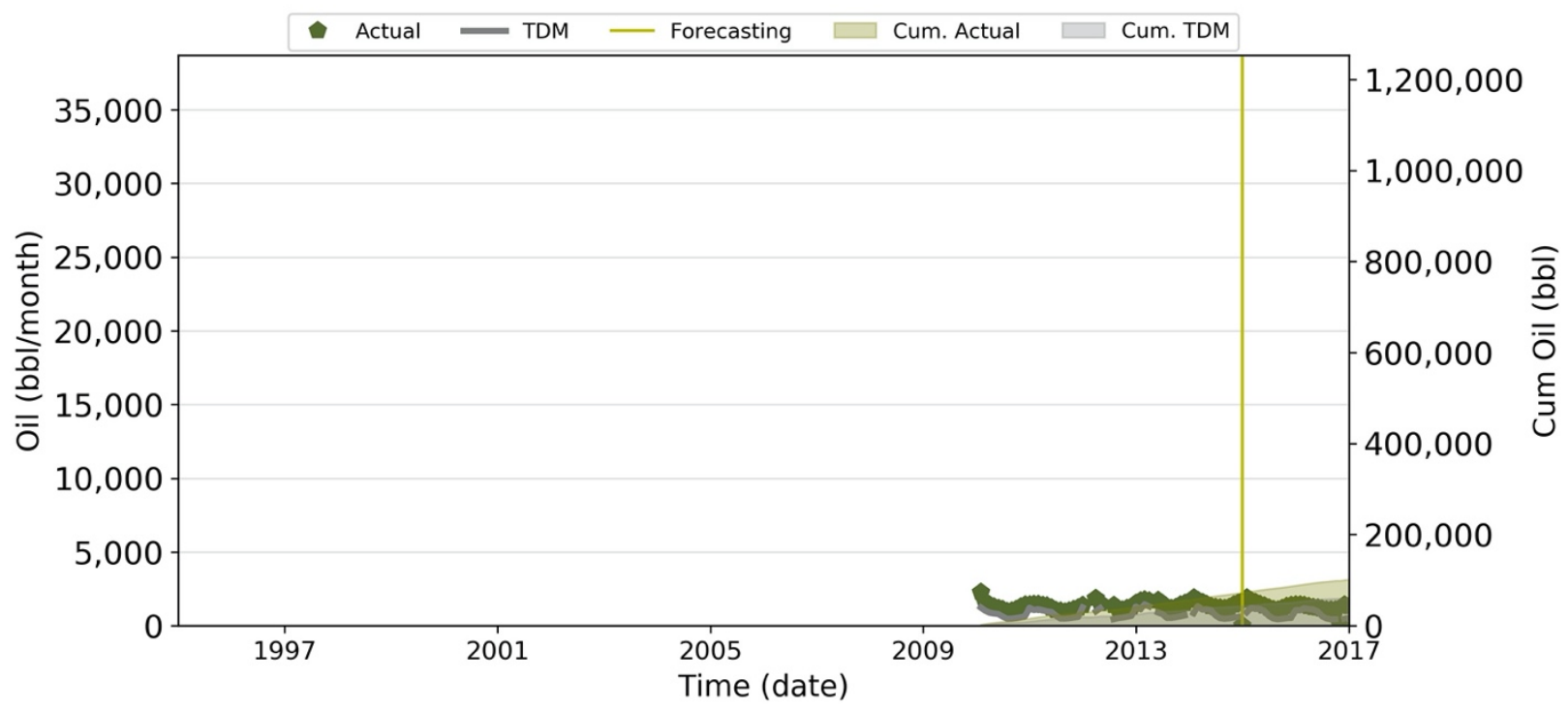


Oil: P-57

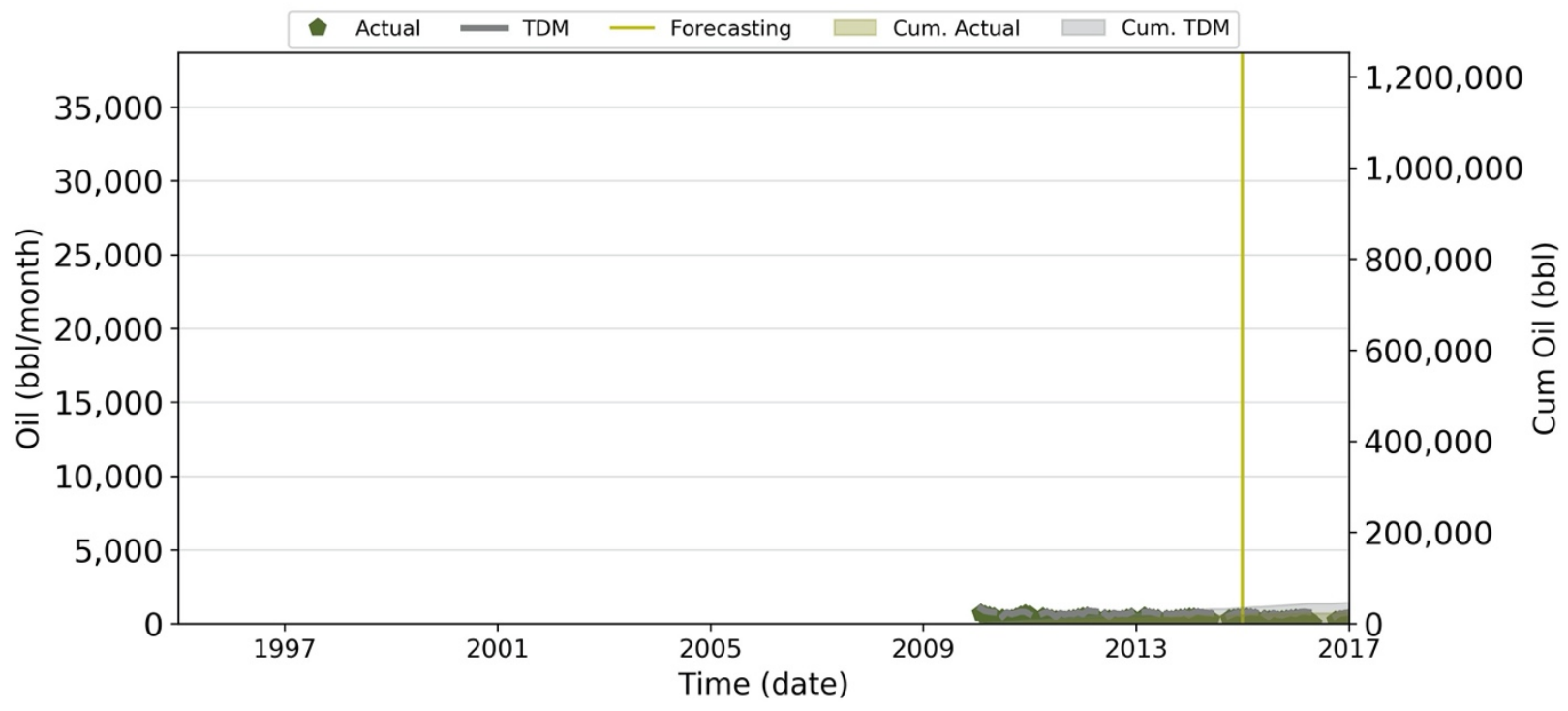


Gas Results

Gas: P-01

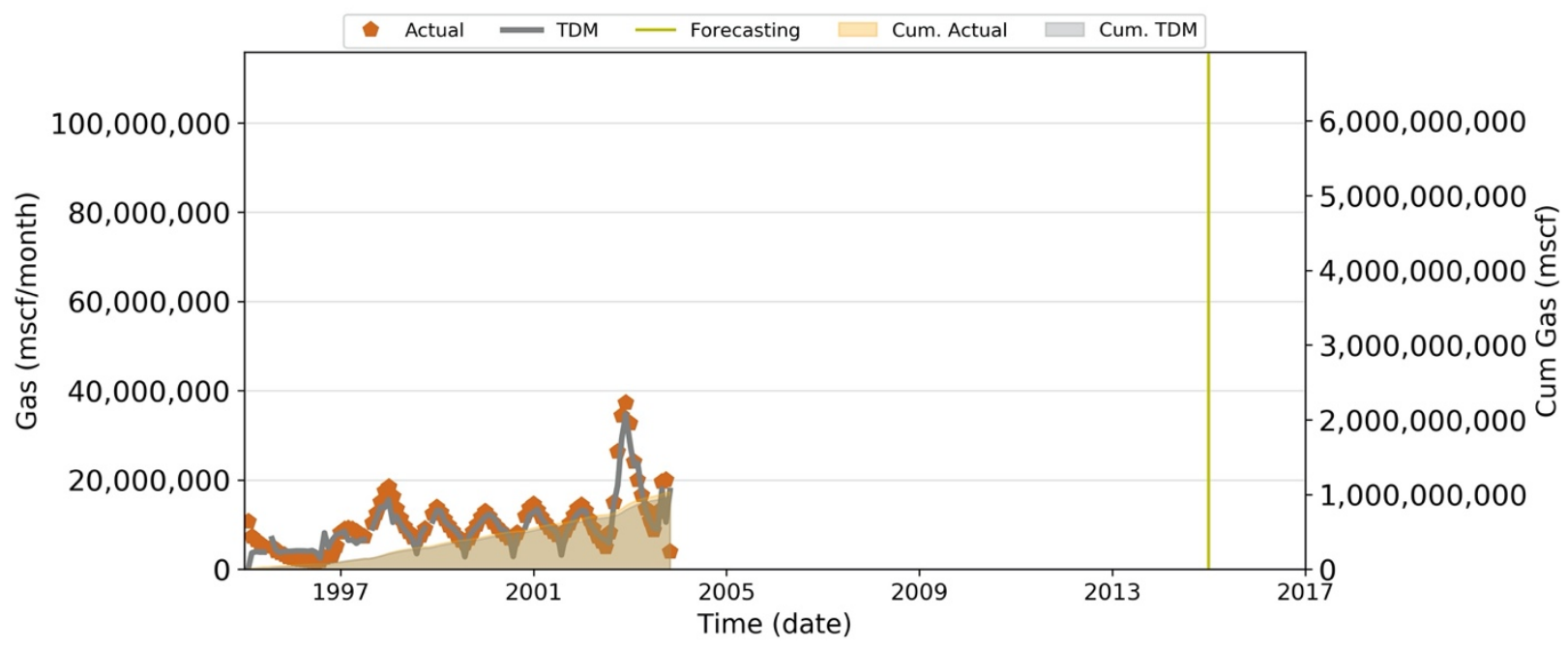

Gas: P-02

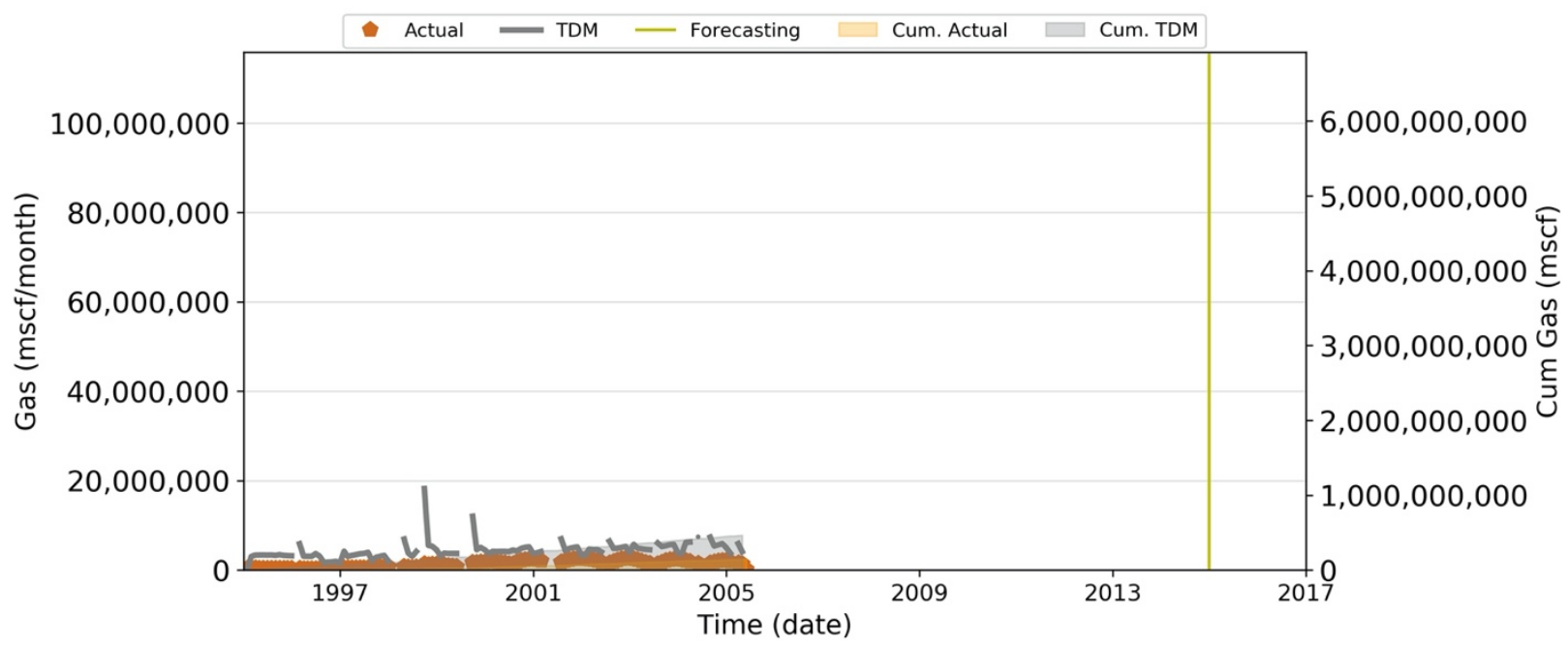


Gas: P-03

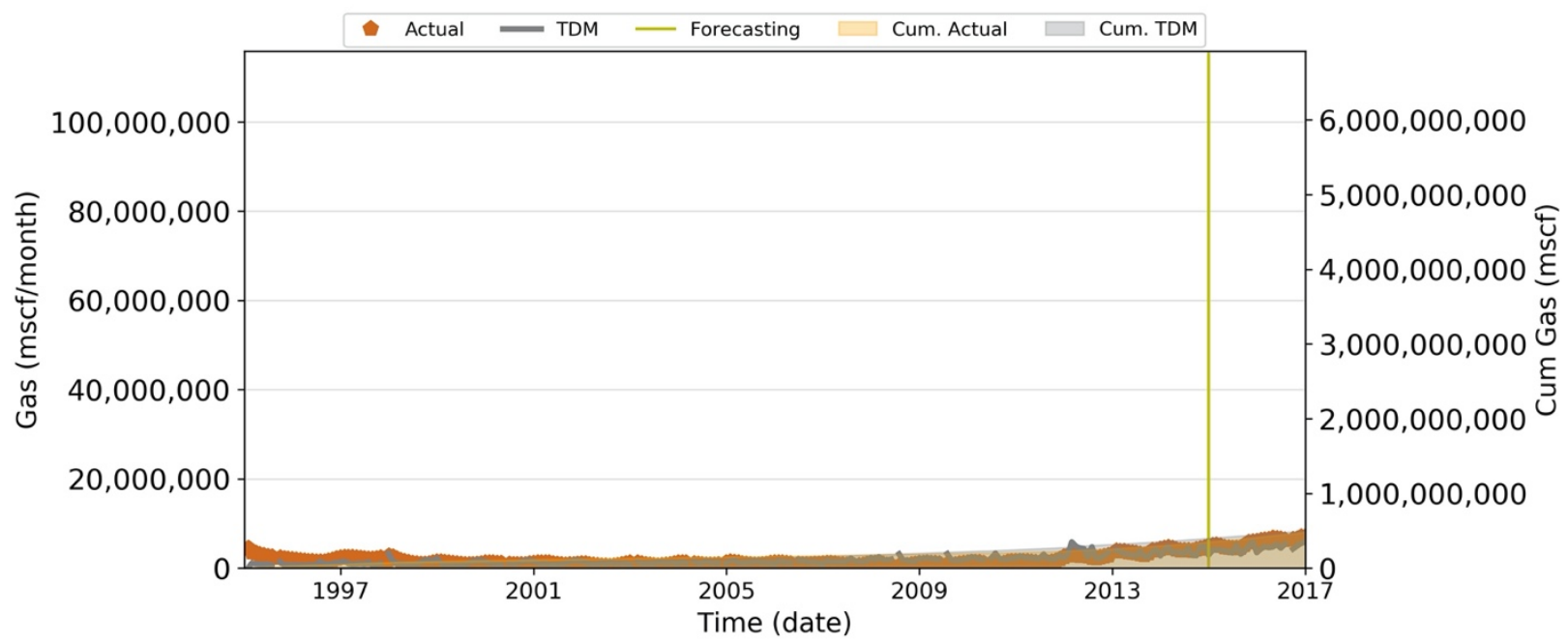

Gas: P-04

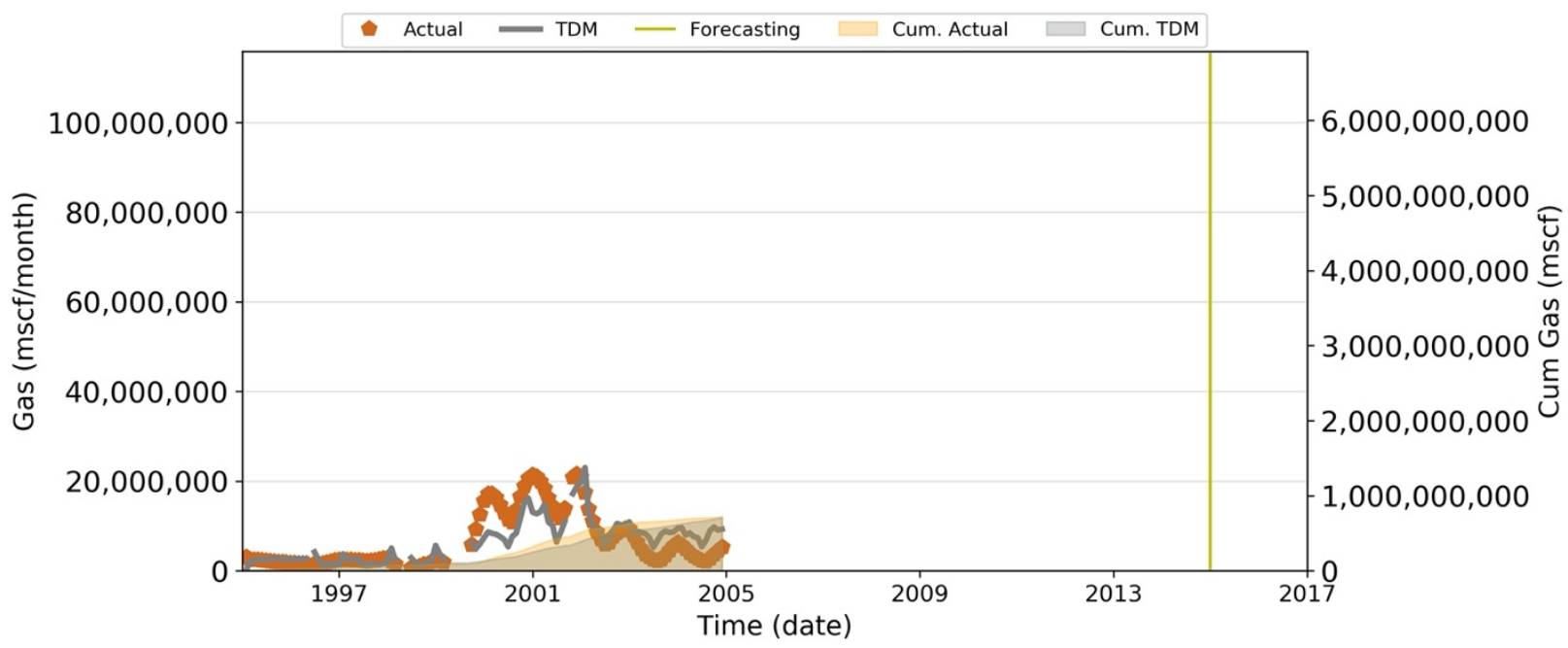

Gas: P-05

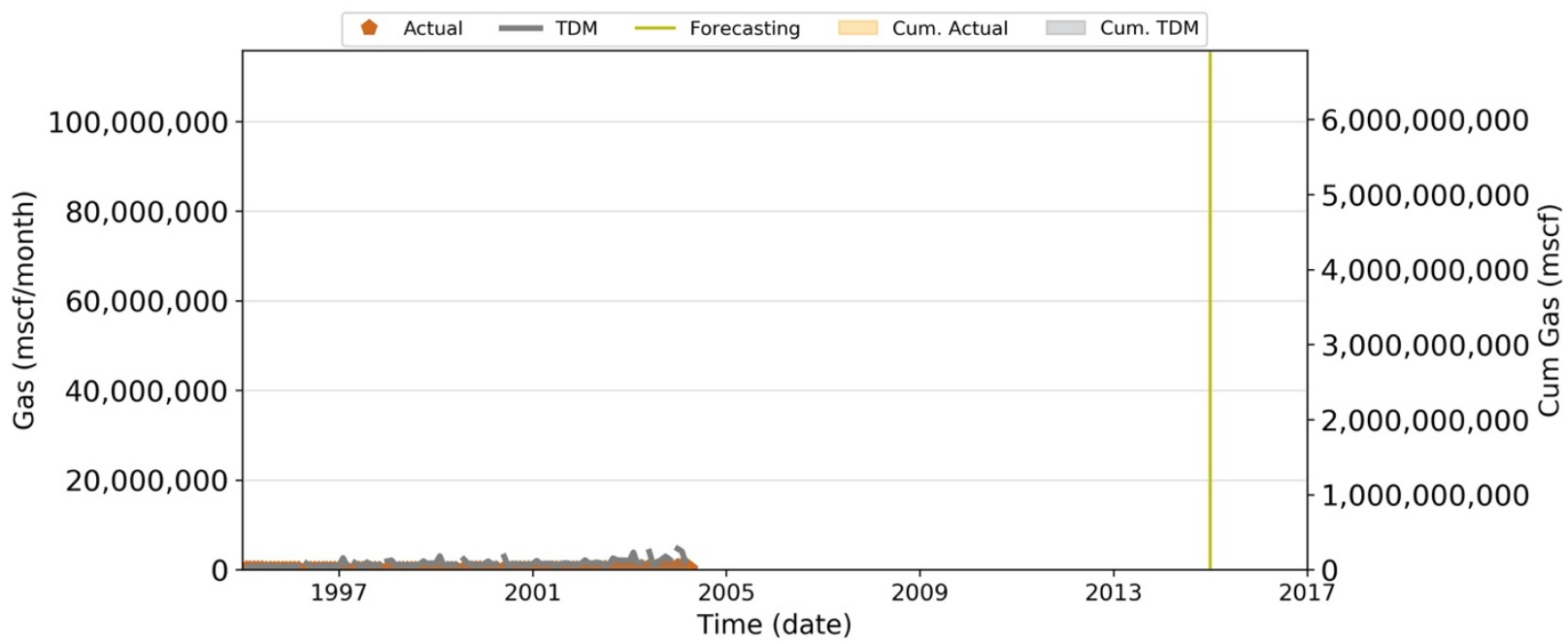


Gas: P-06

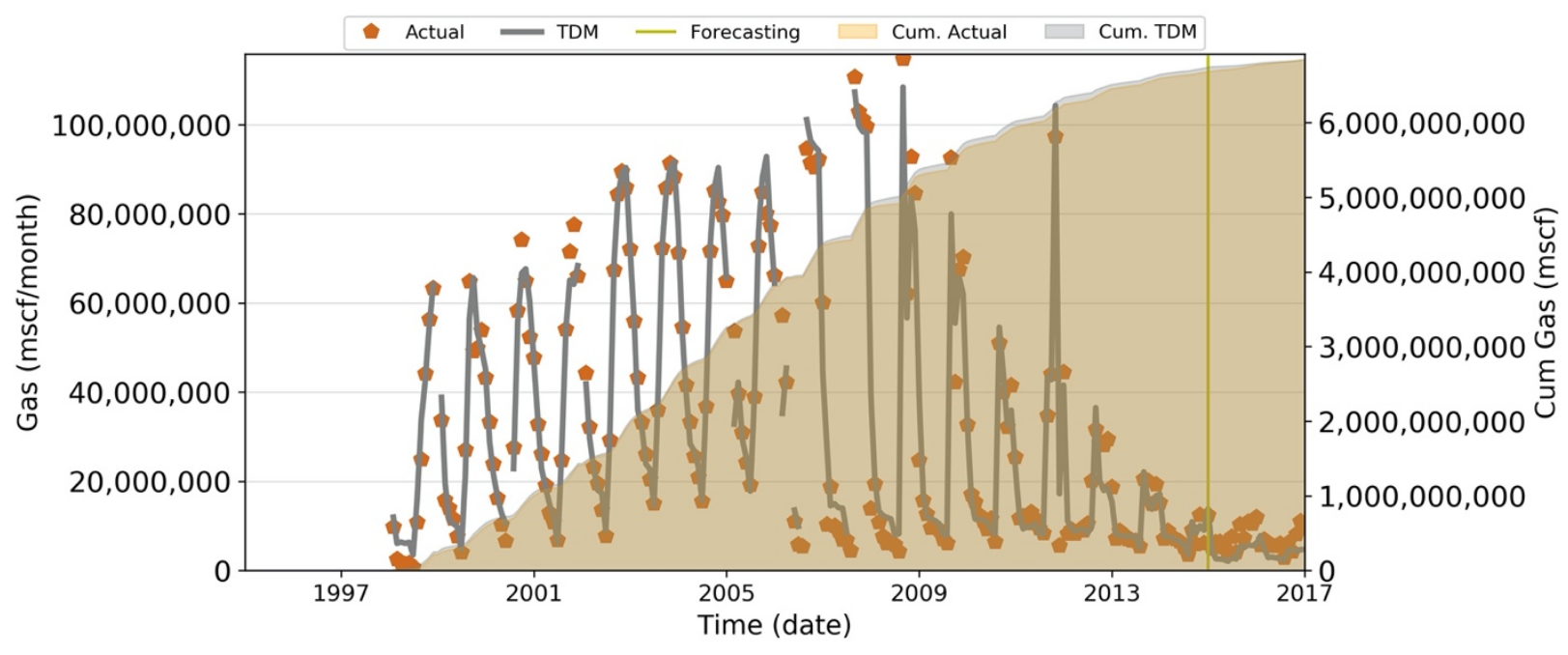

Gas: P-07

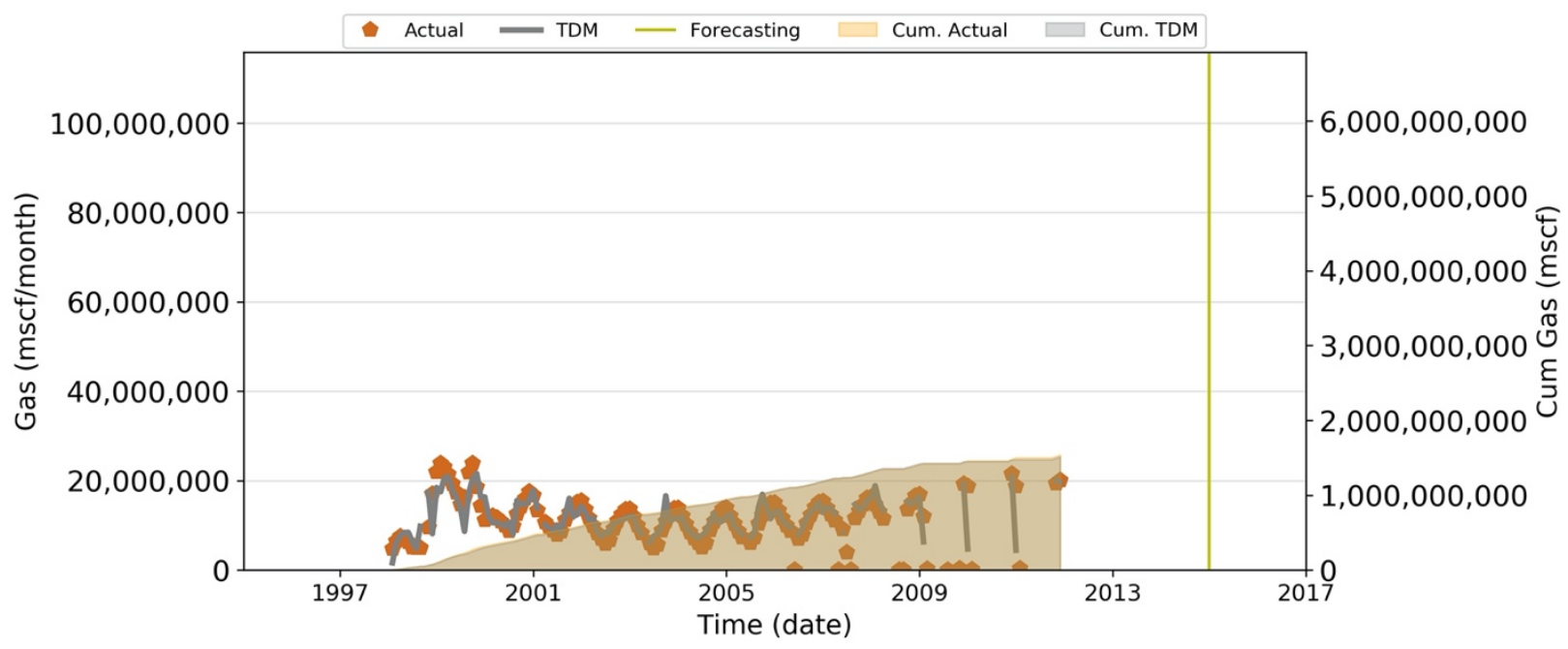

Gas: P-09

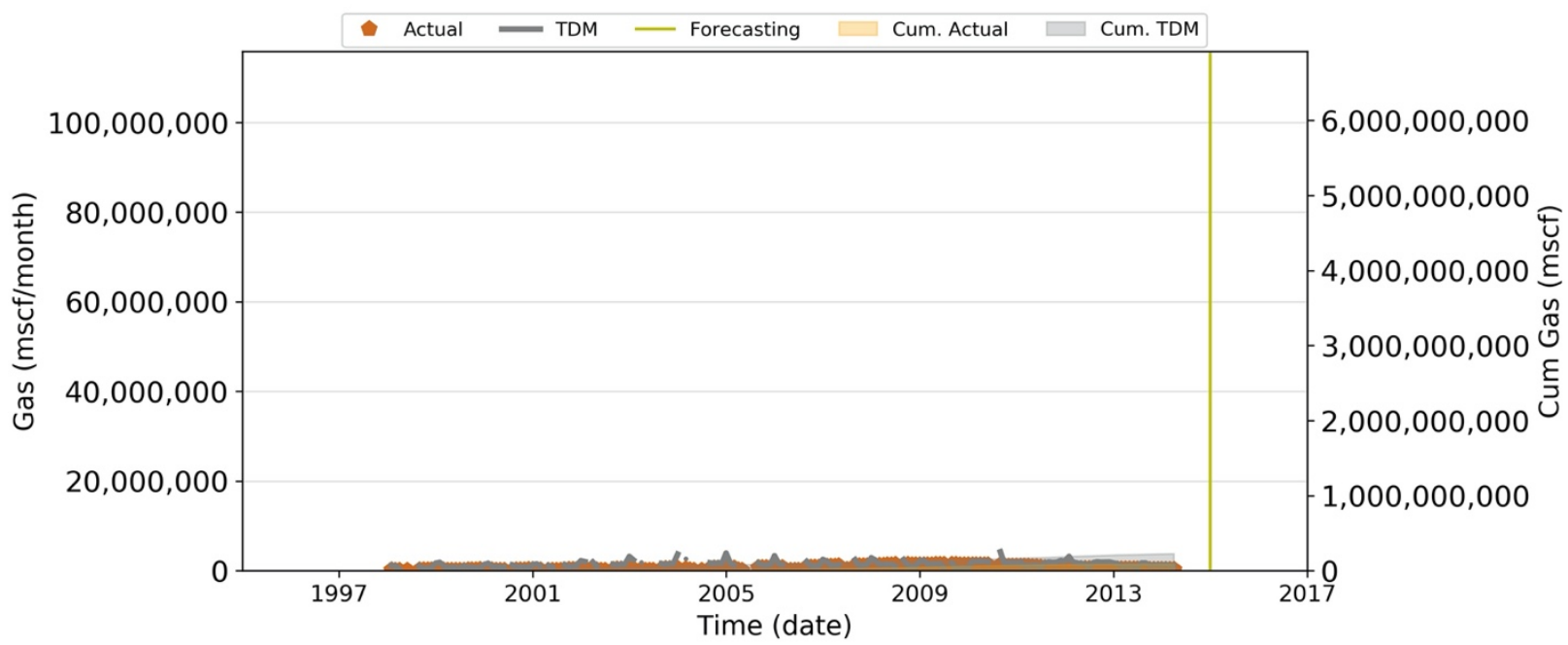


Gas: P-10

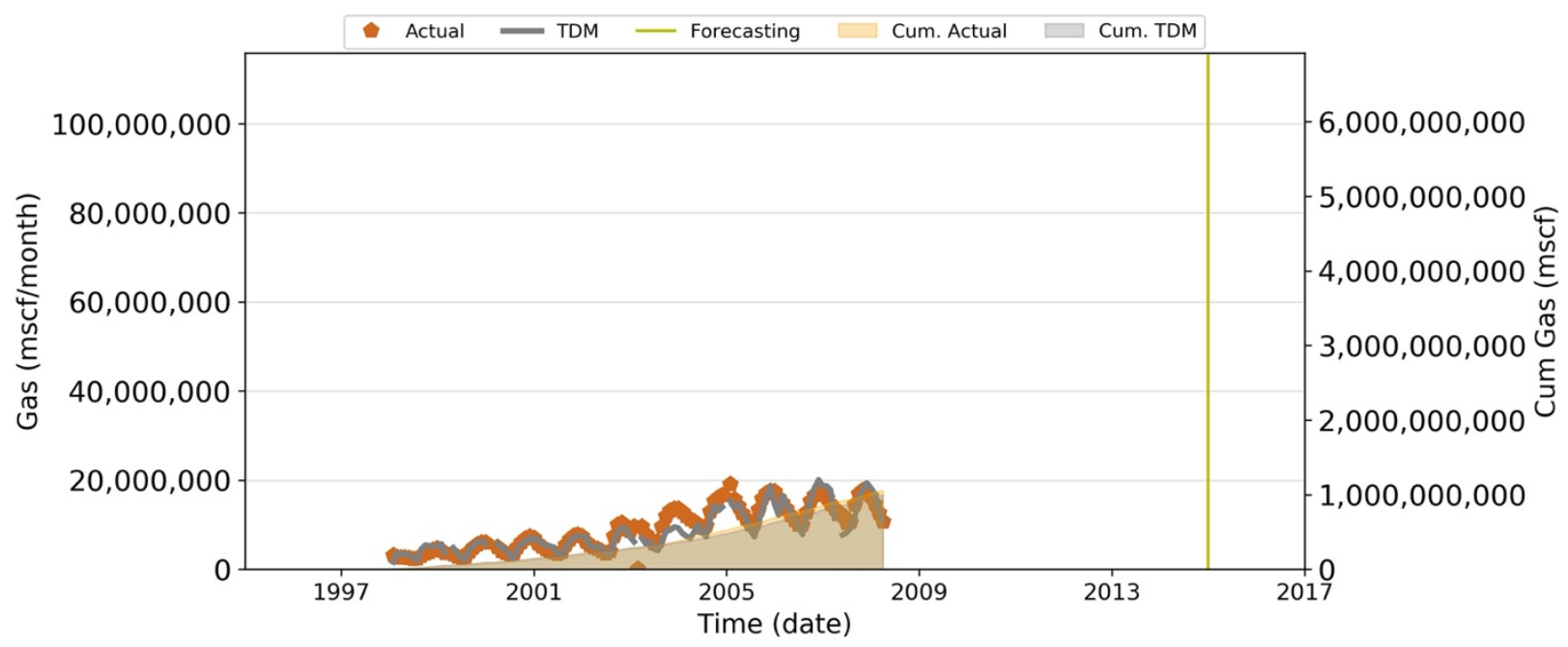

Gas: P-11

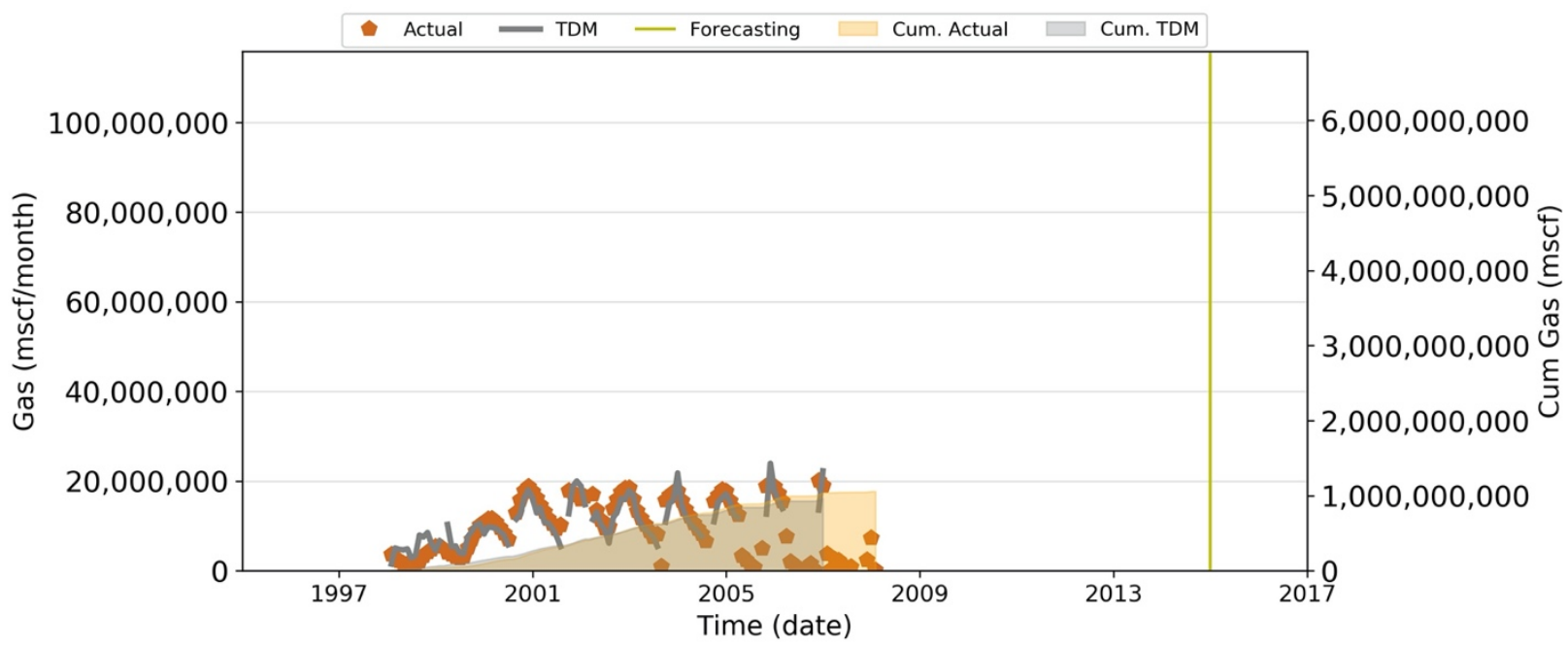

Gas: P-13

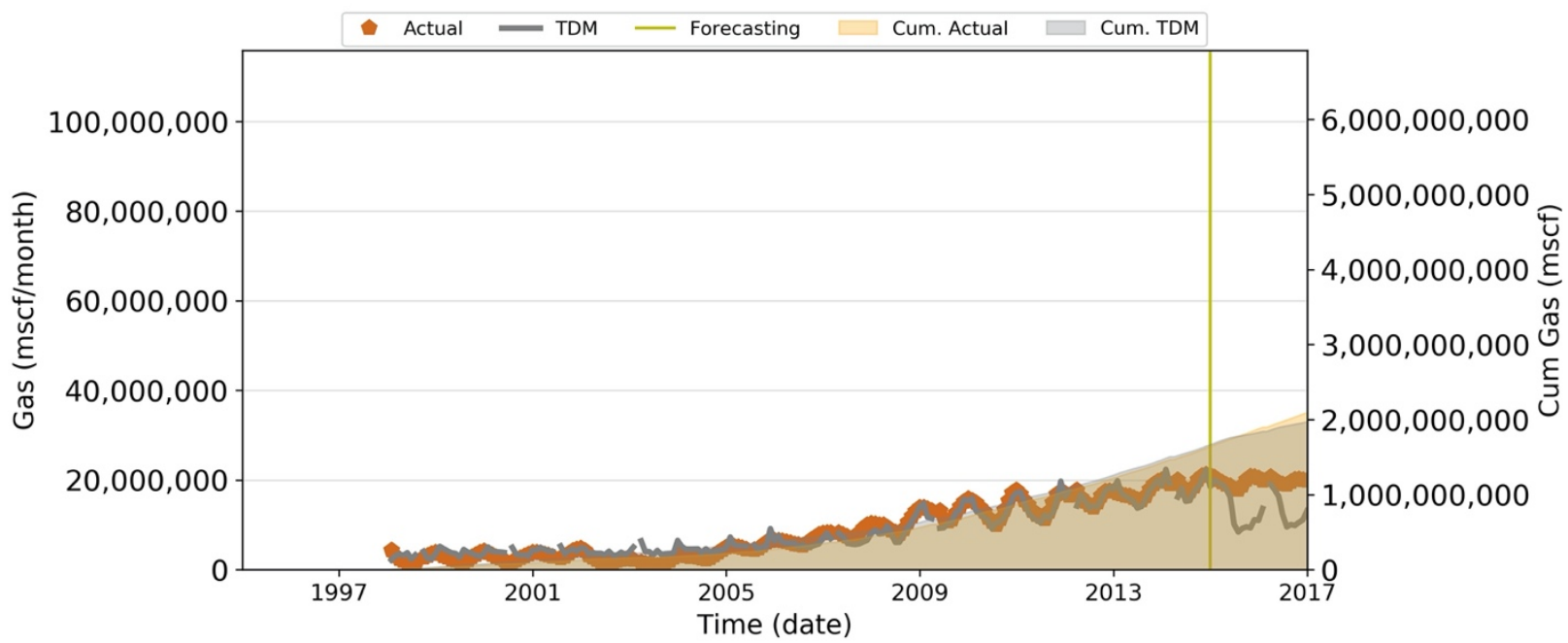


Gas: P-14

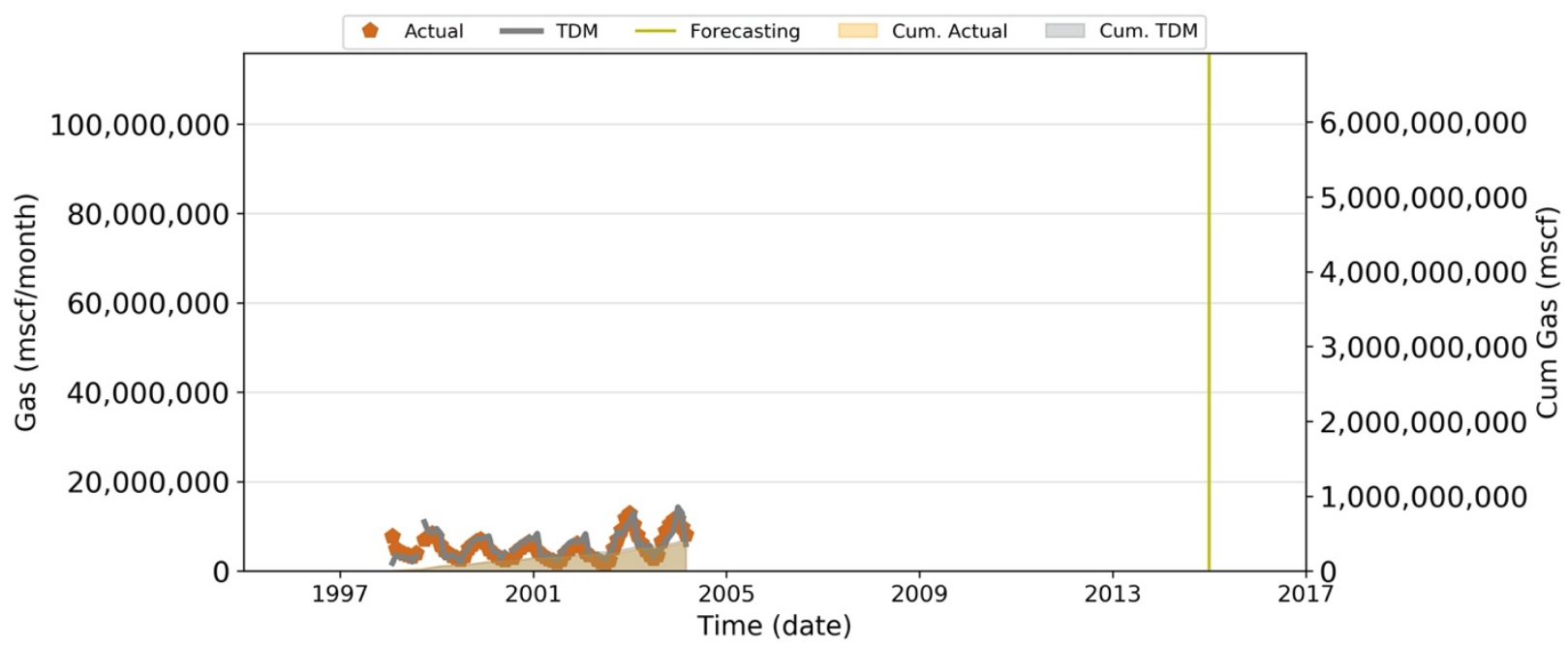

Gas: P-15

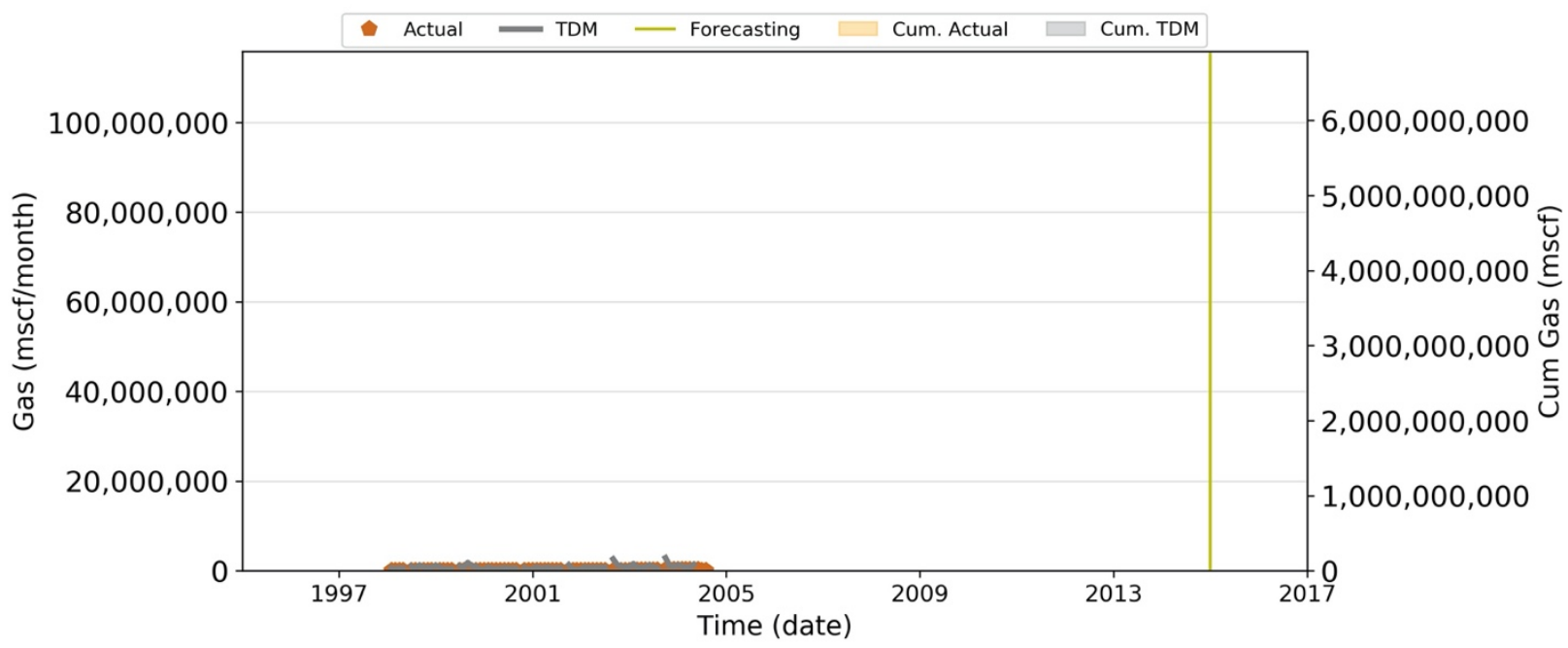

Gas: P-16

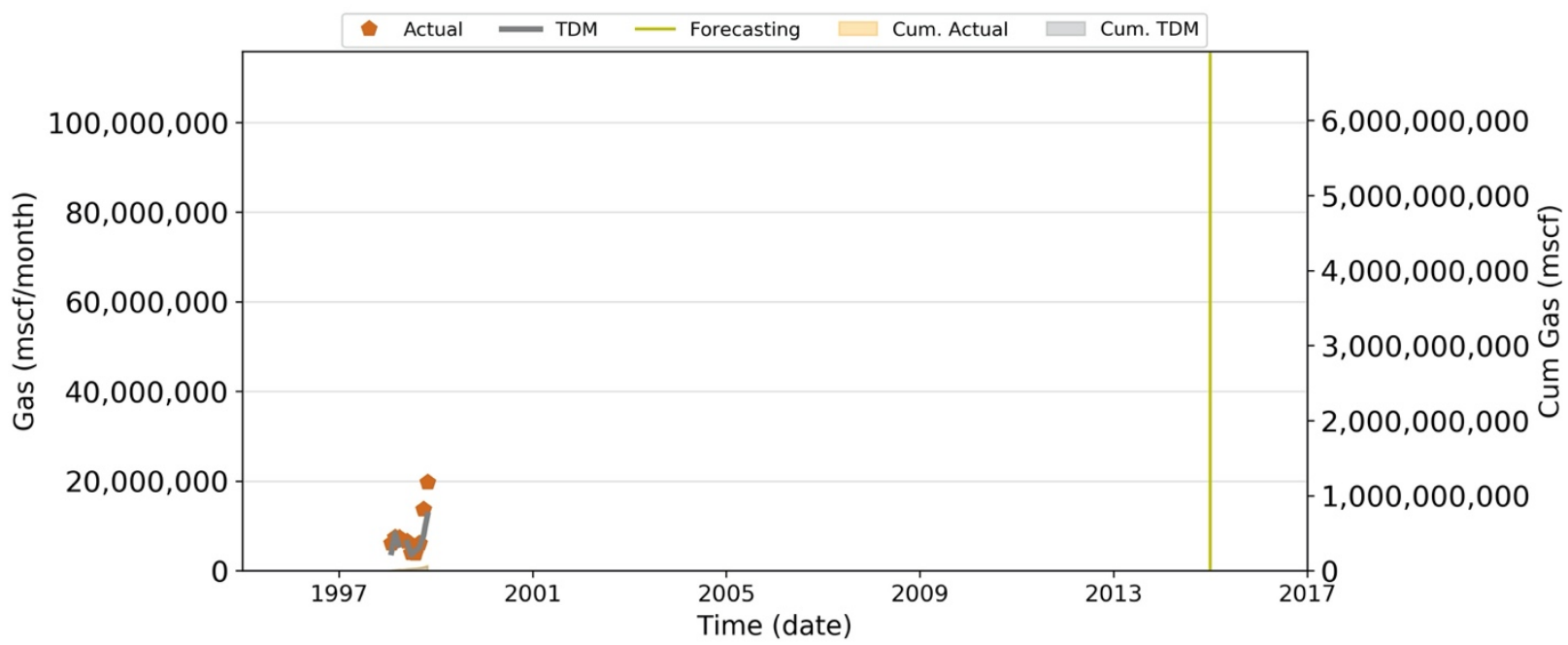


Gas: P-17

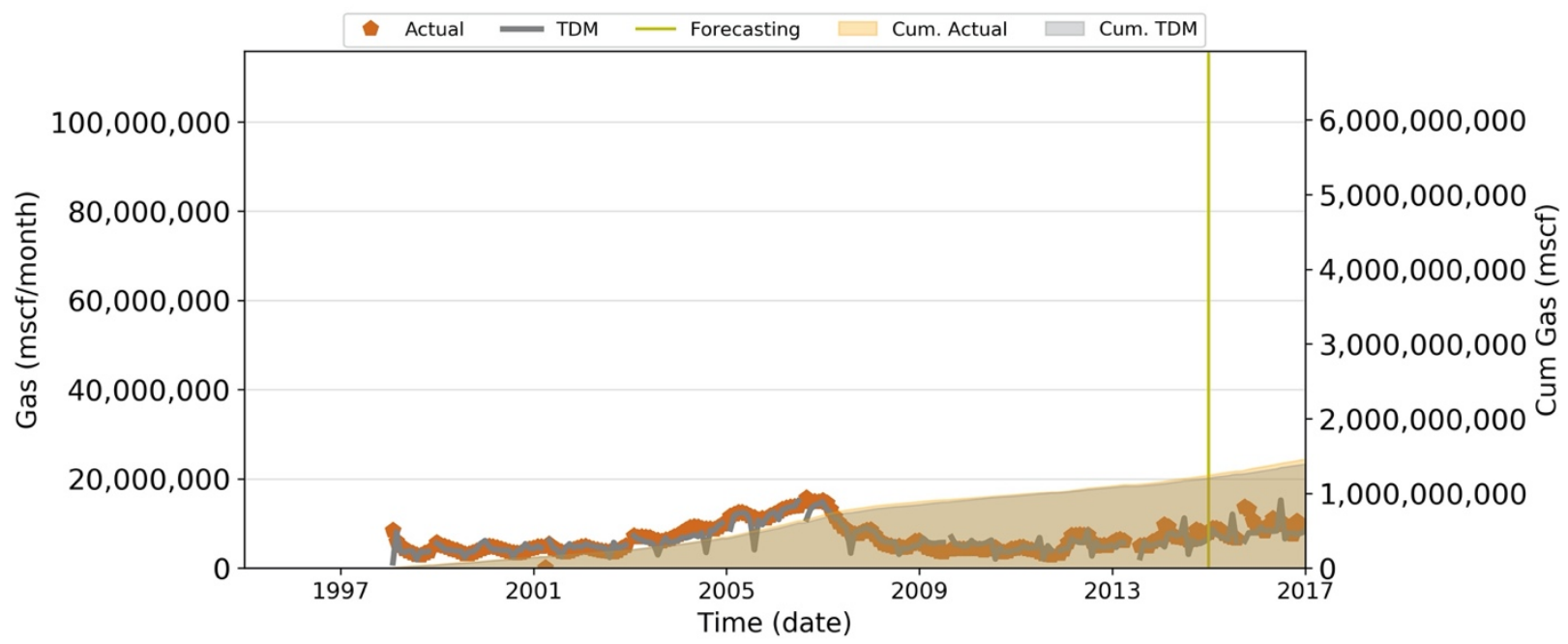

Gas: P-18

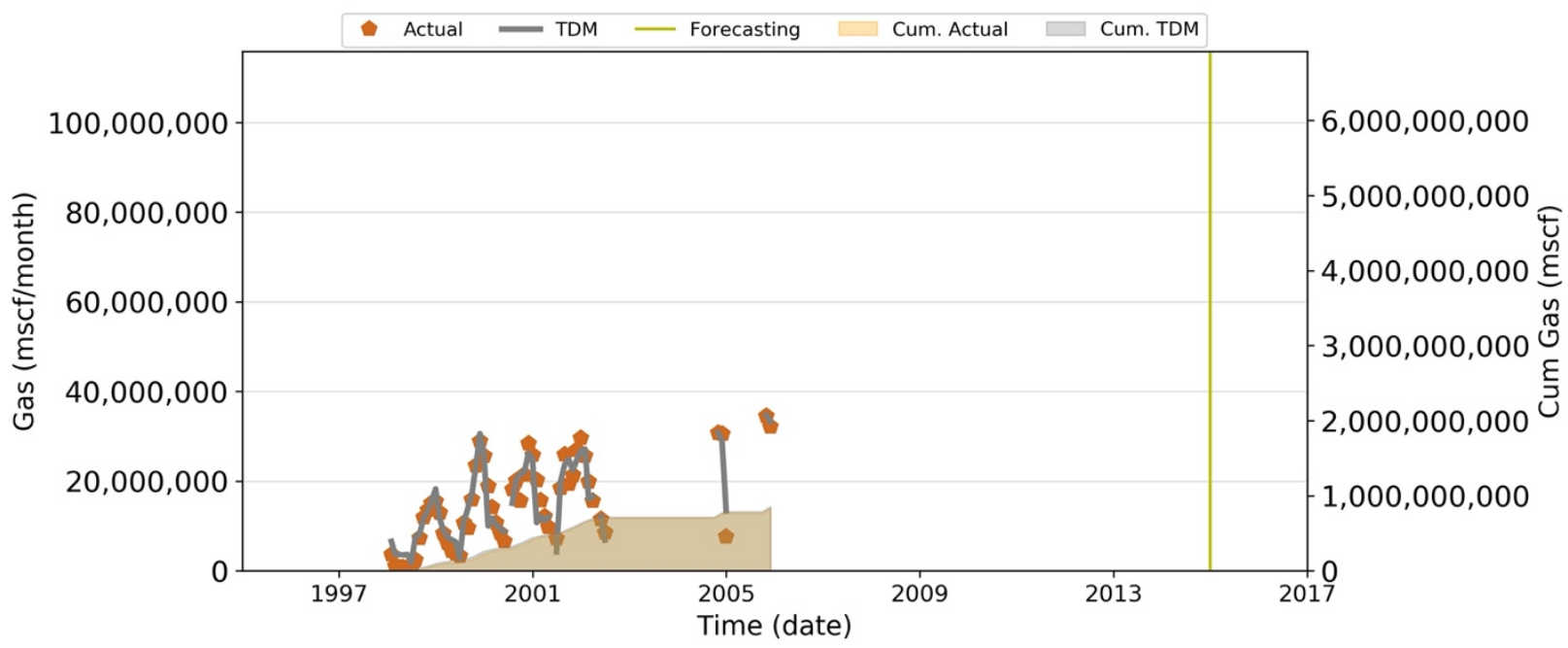

Gas: P-19

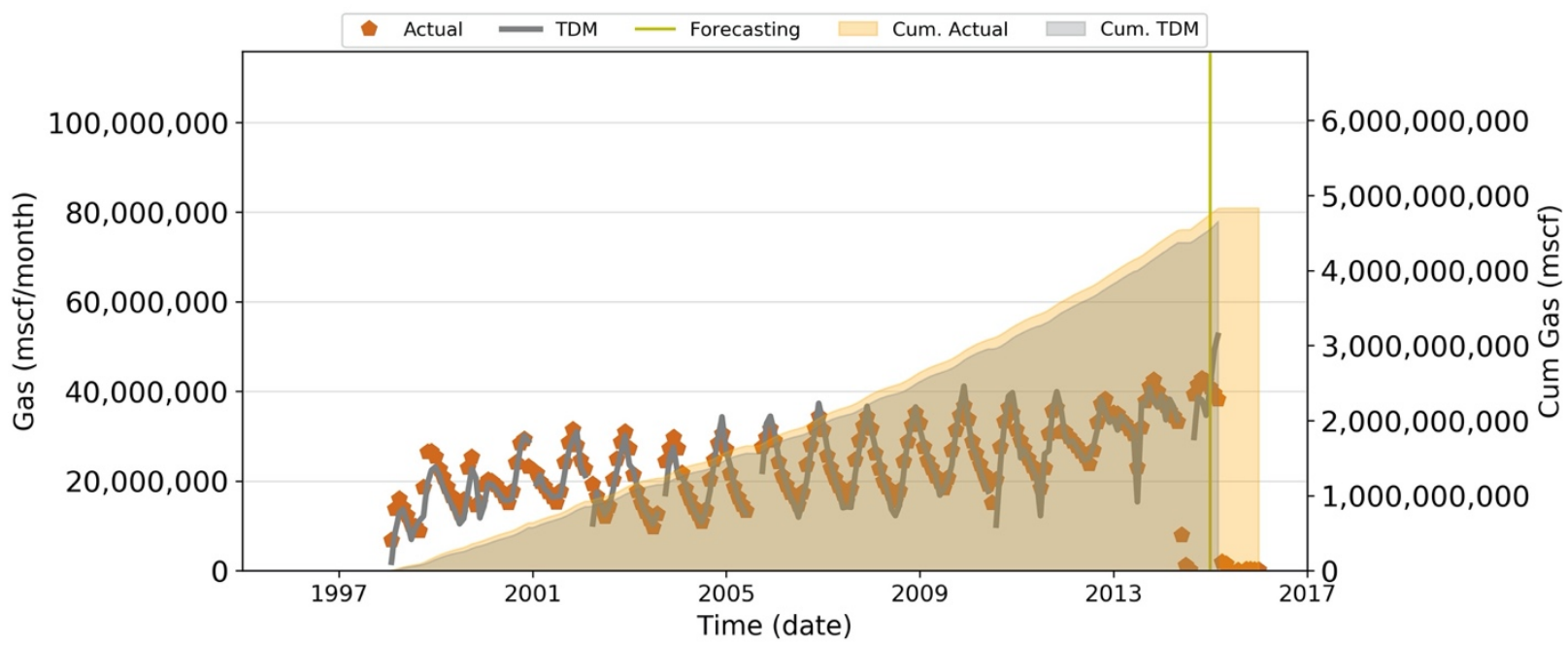


Gas: P-20

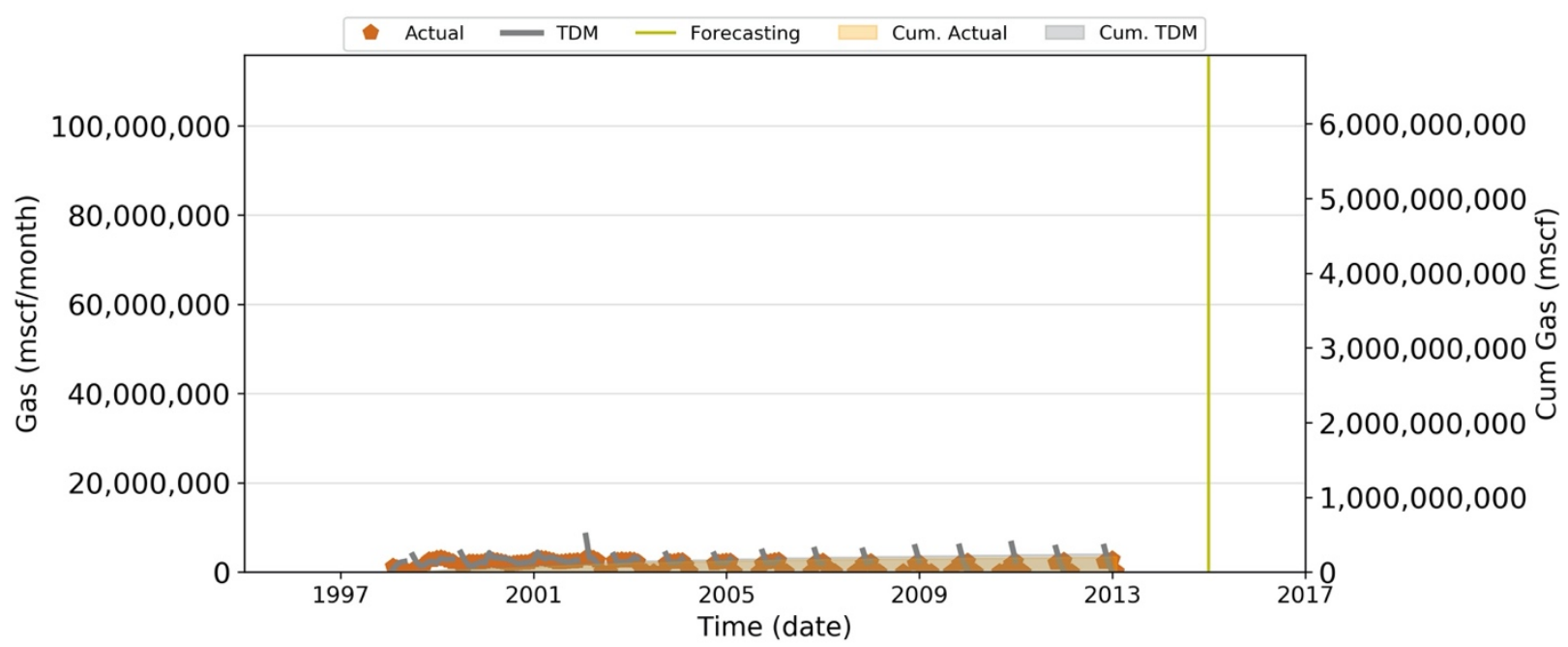

Gas: P-21

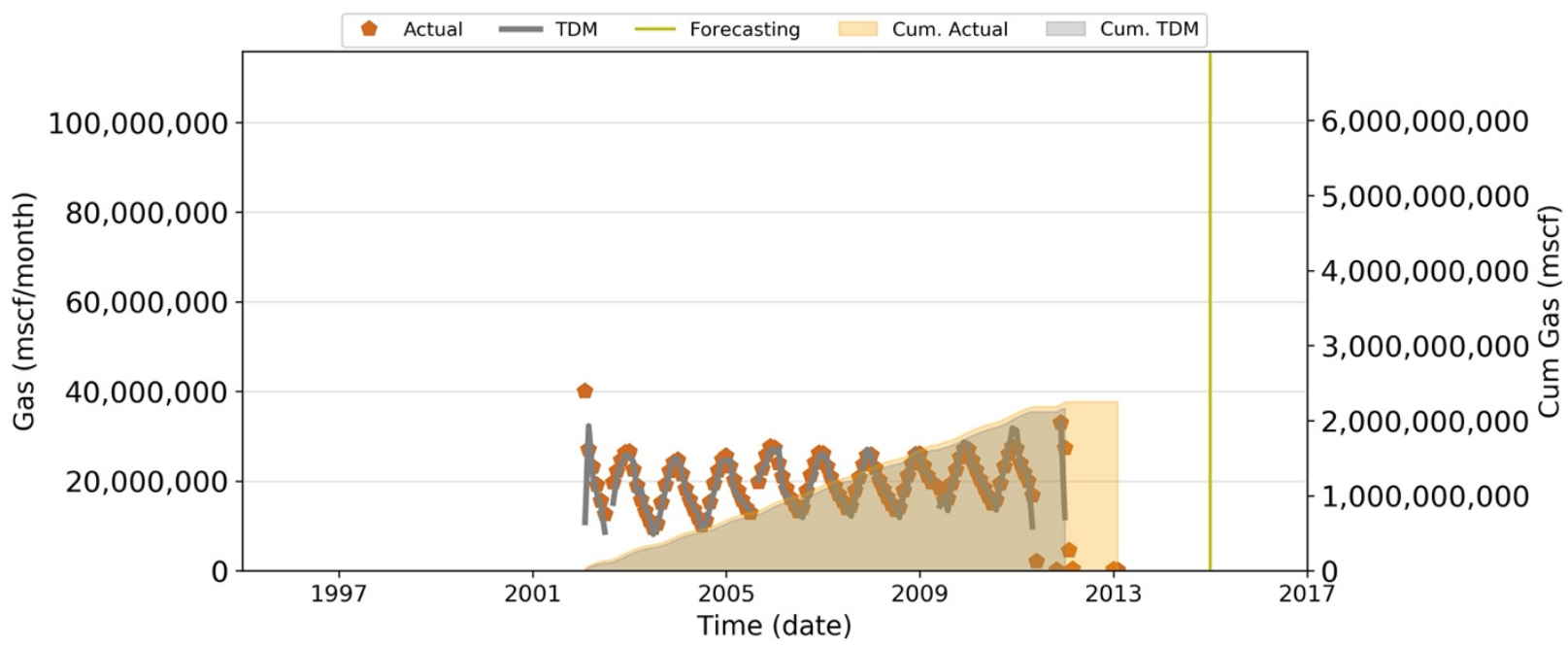

Gas: P-22

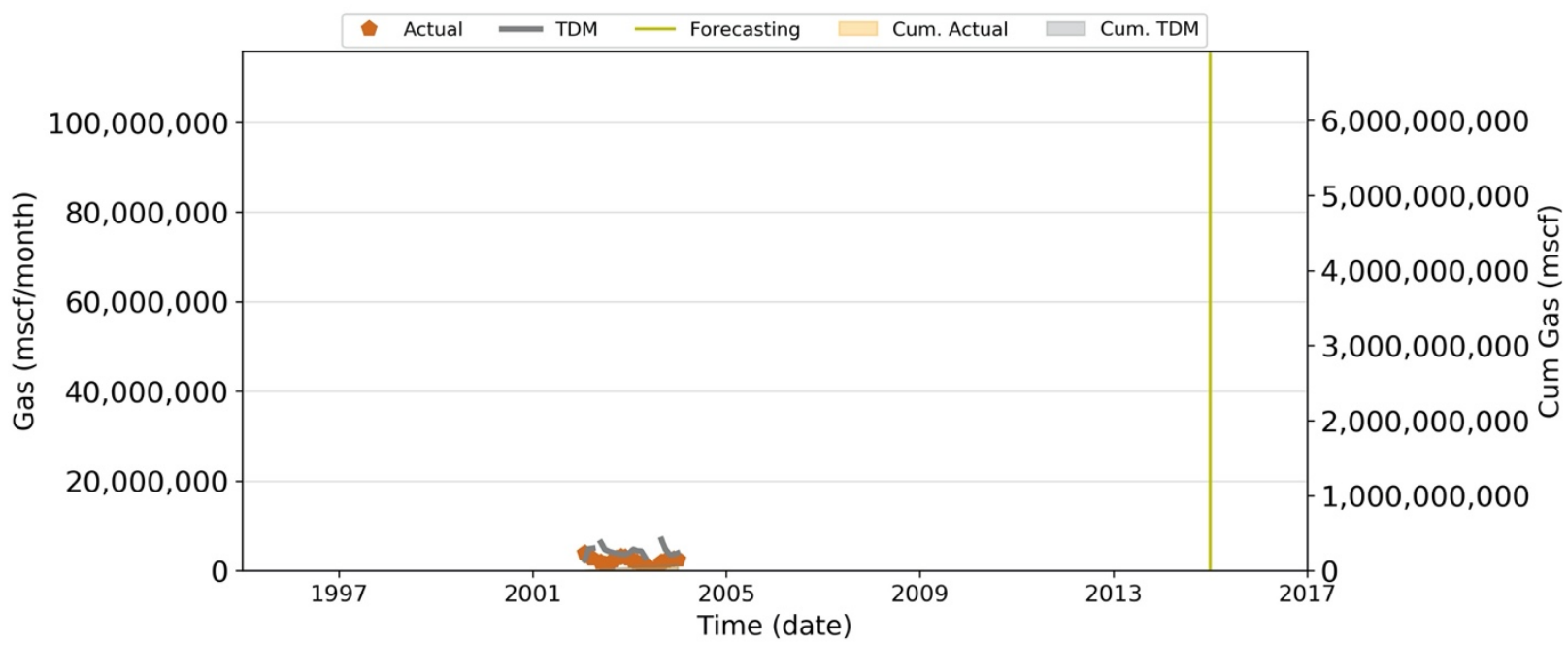


Gas: P-23

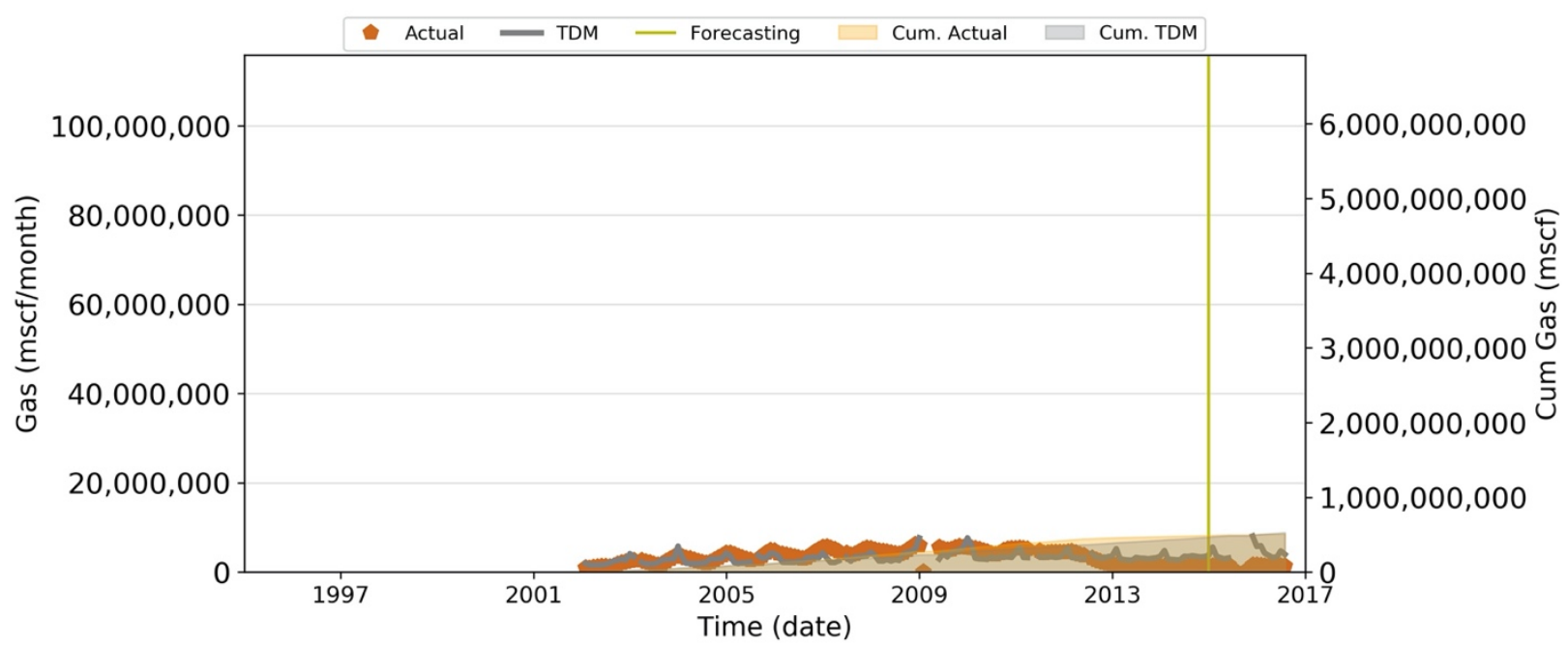

Gas: P-24

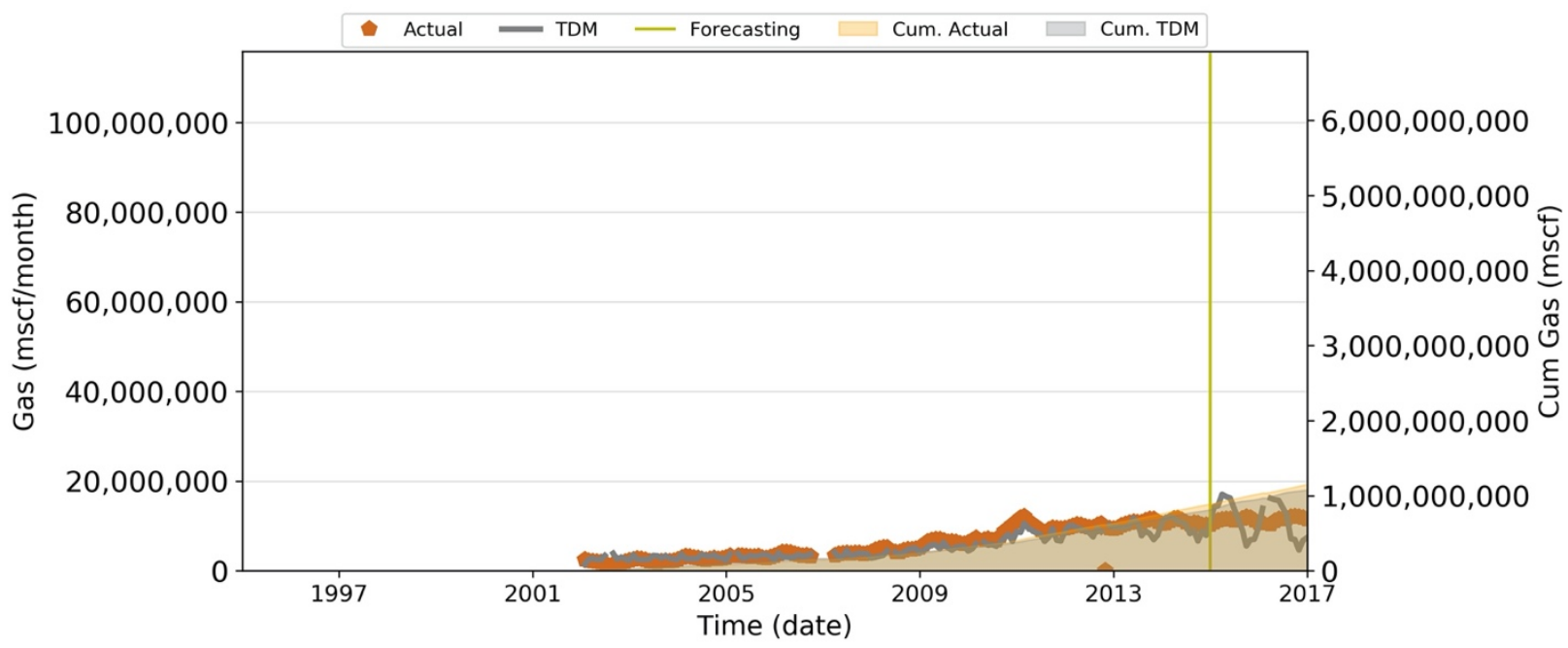

Gas: P-25

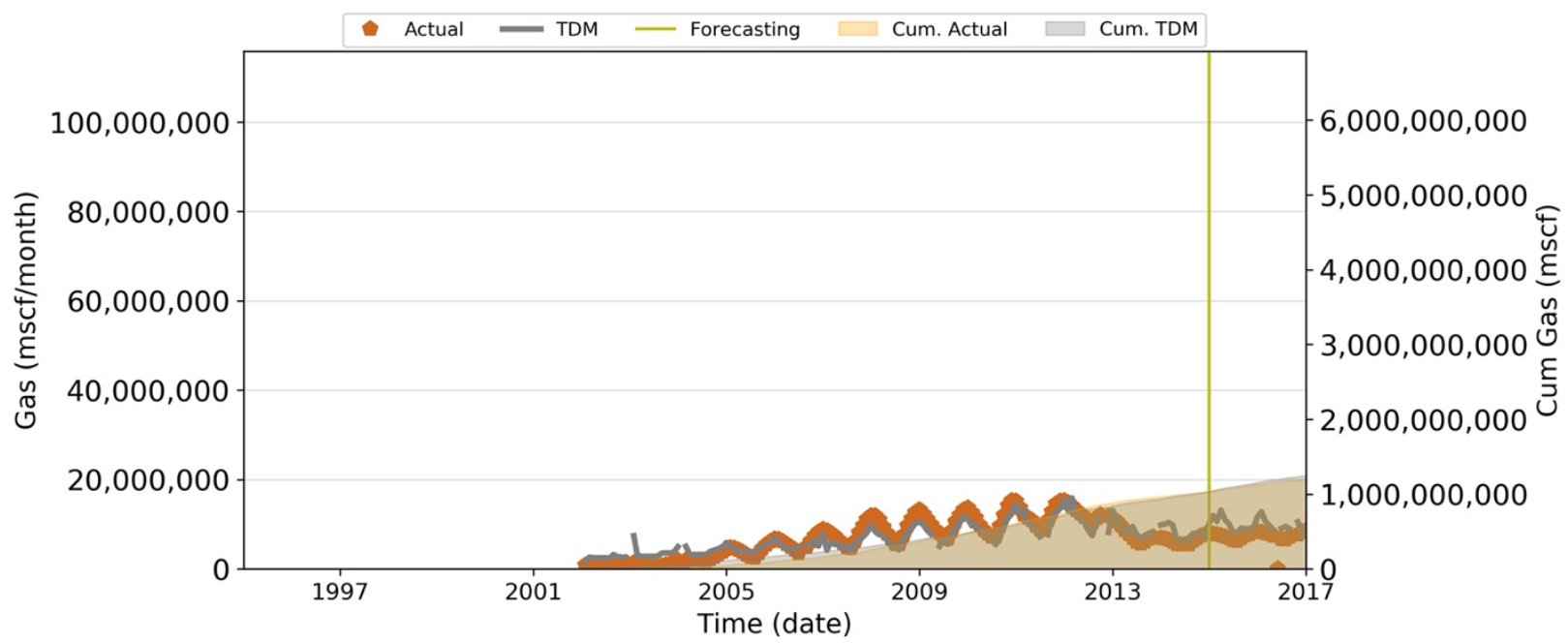


Gas: P-26

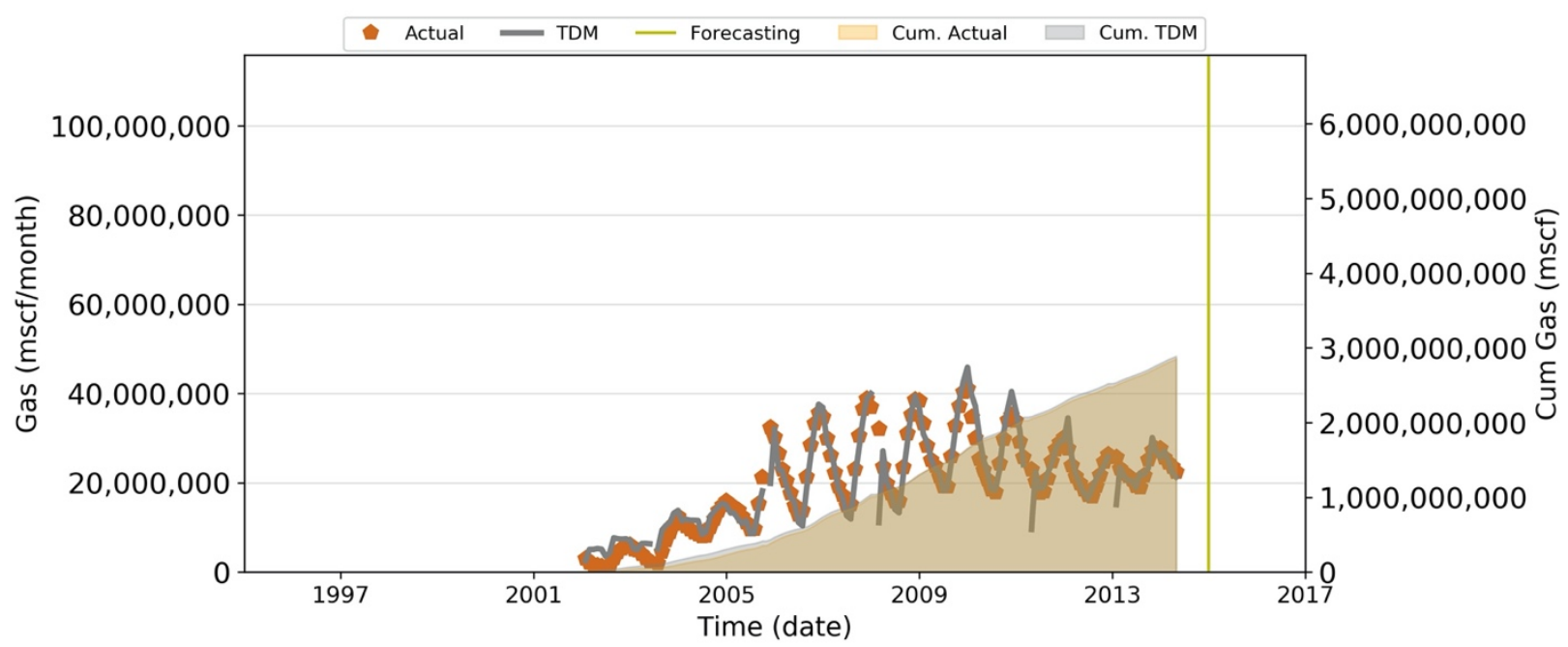

Gas: P-27

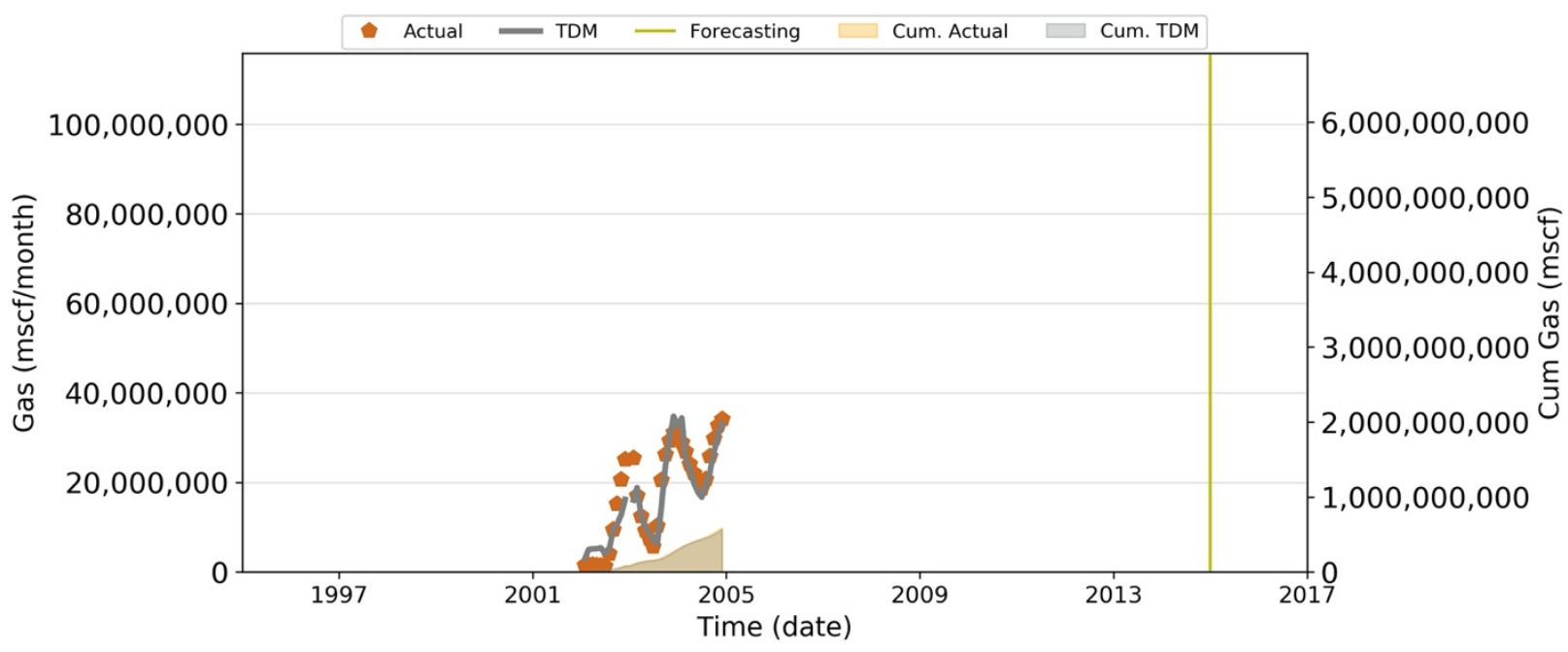

Gas: P-28

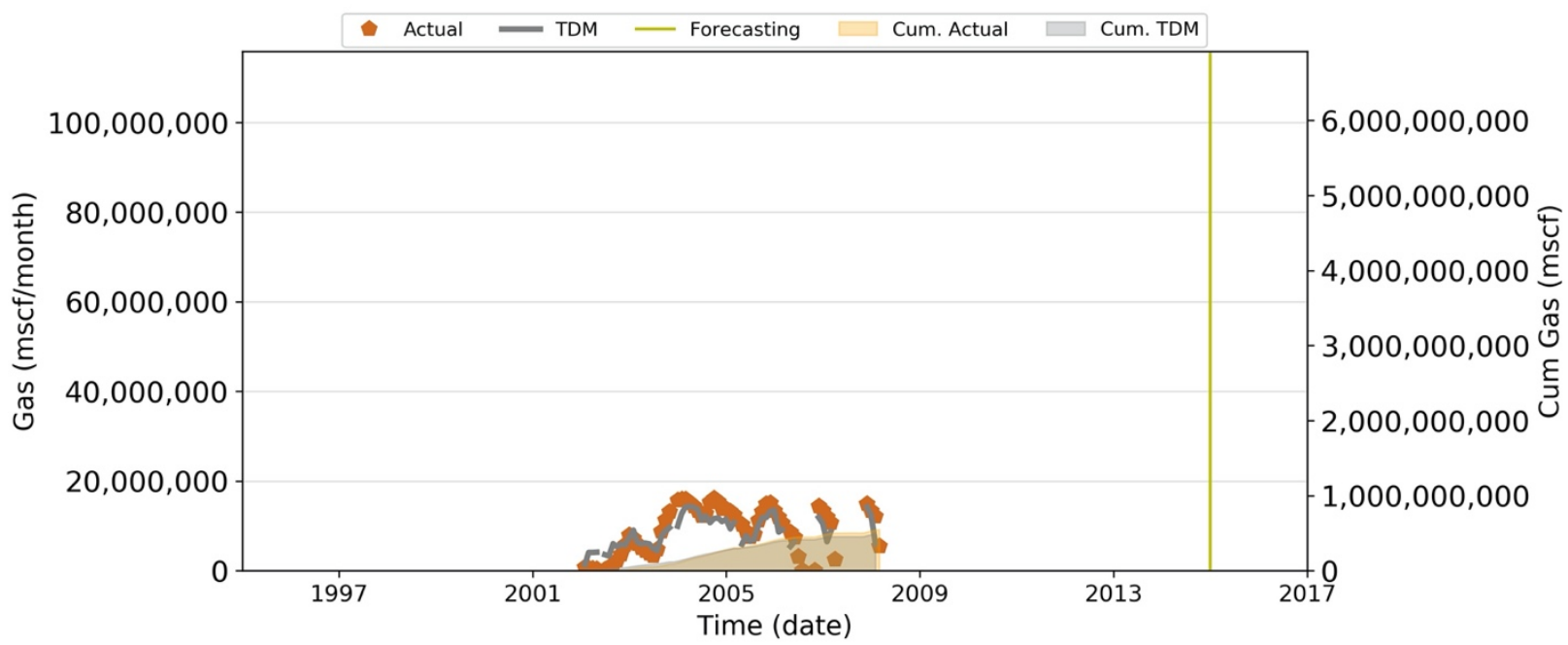


Gas: P-29

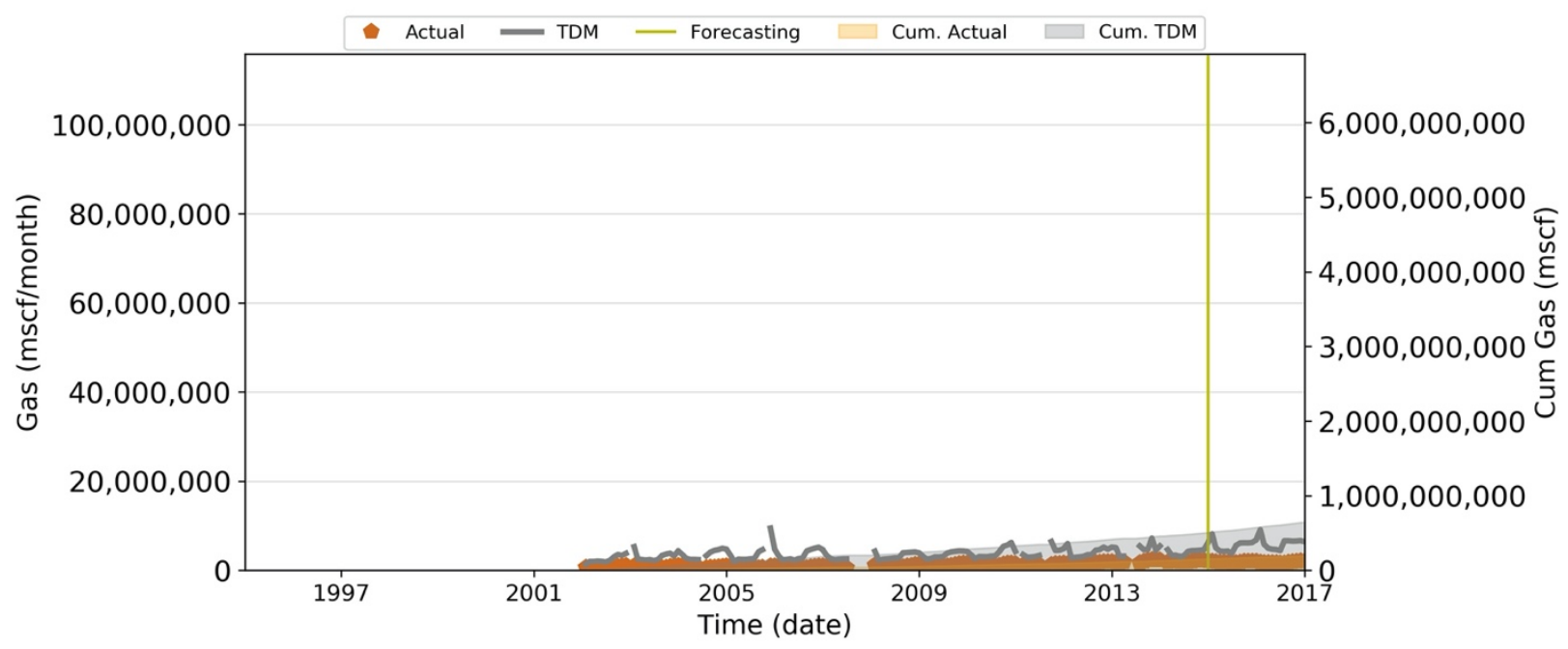

Gas: P-30

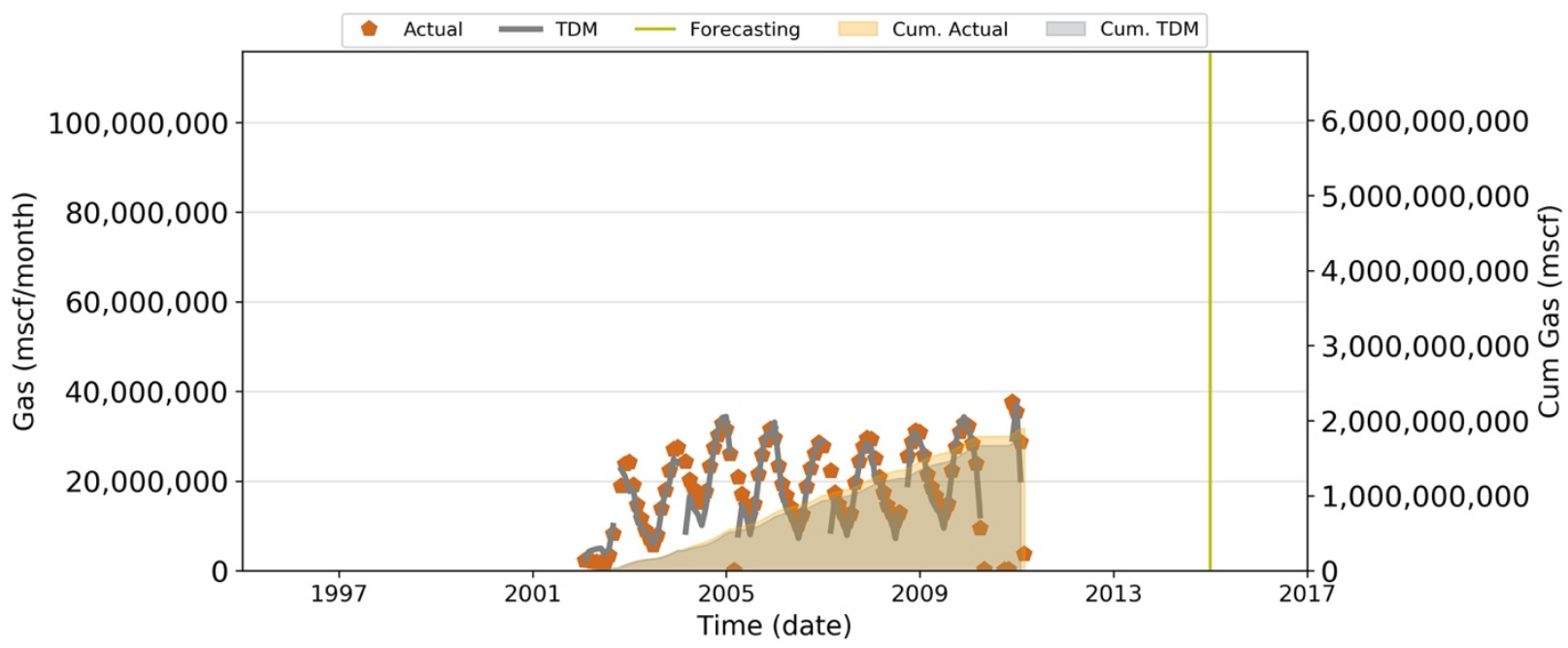

Gas: P-31

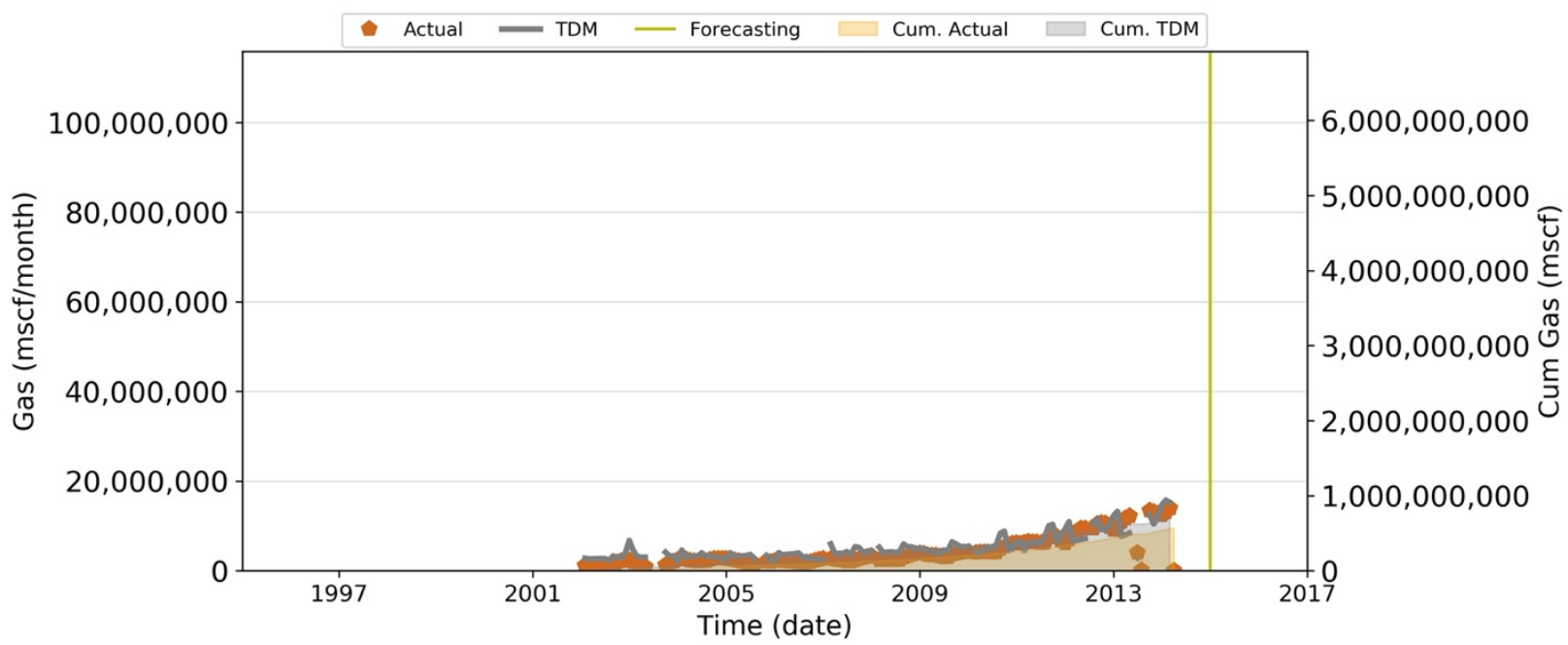


Gas: P-32

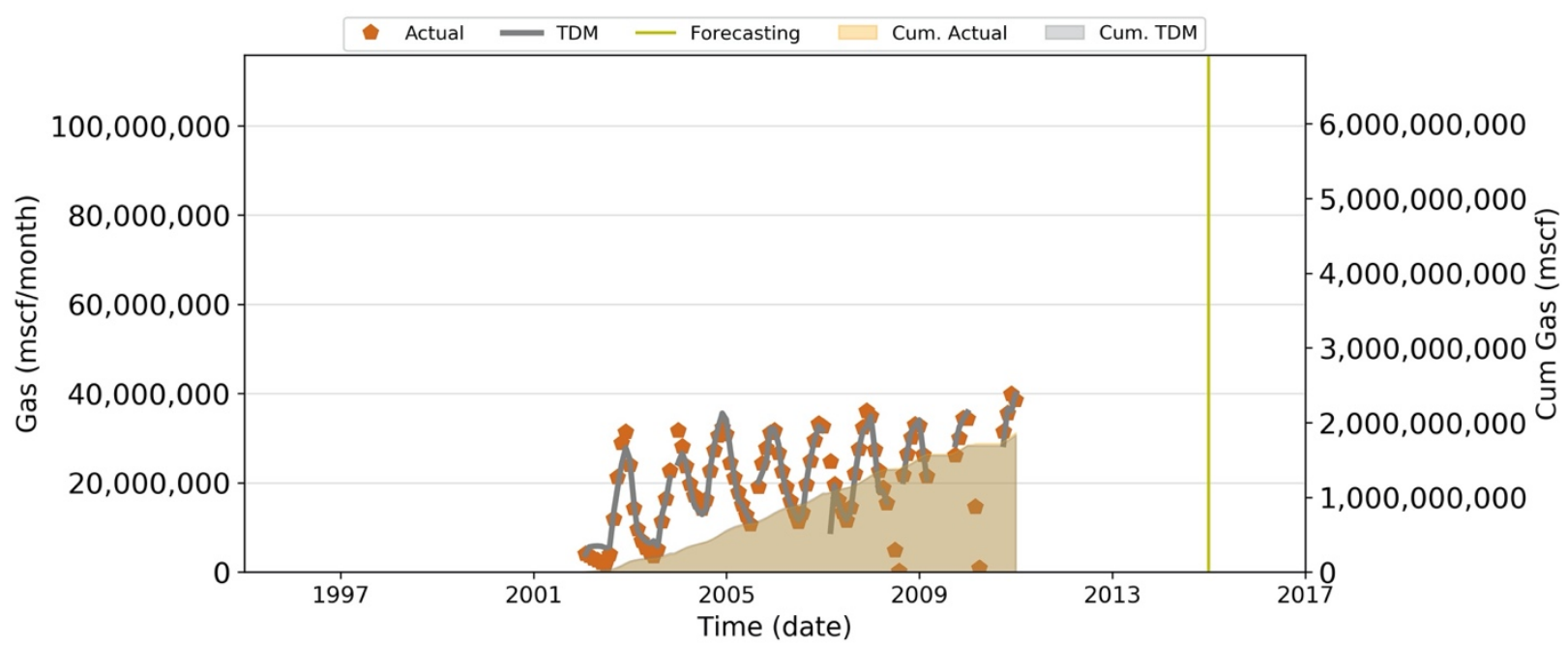

Gas: P-33

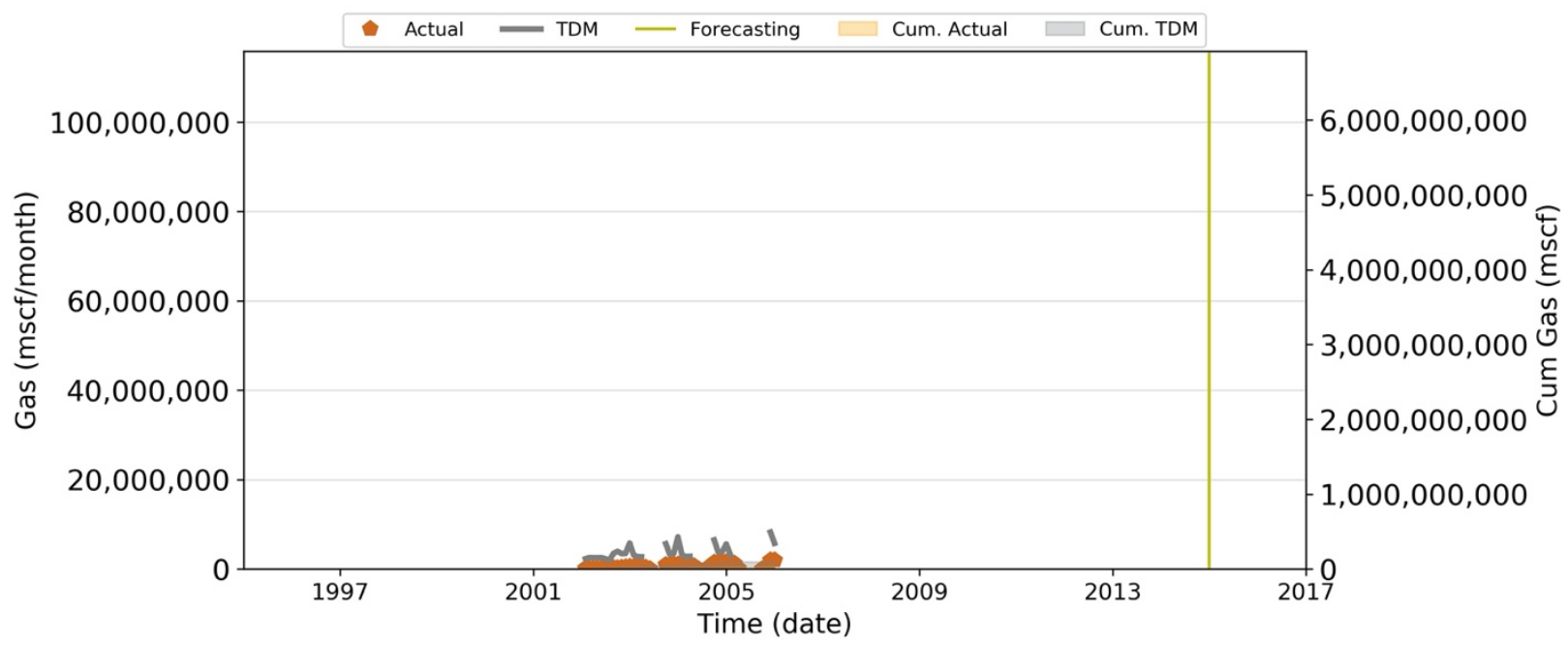

Gas: P-34

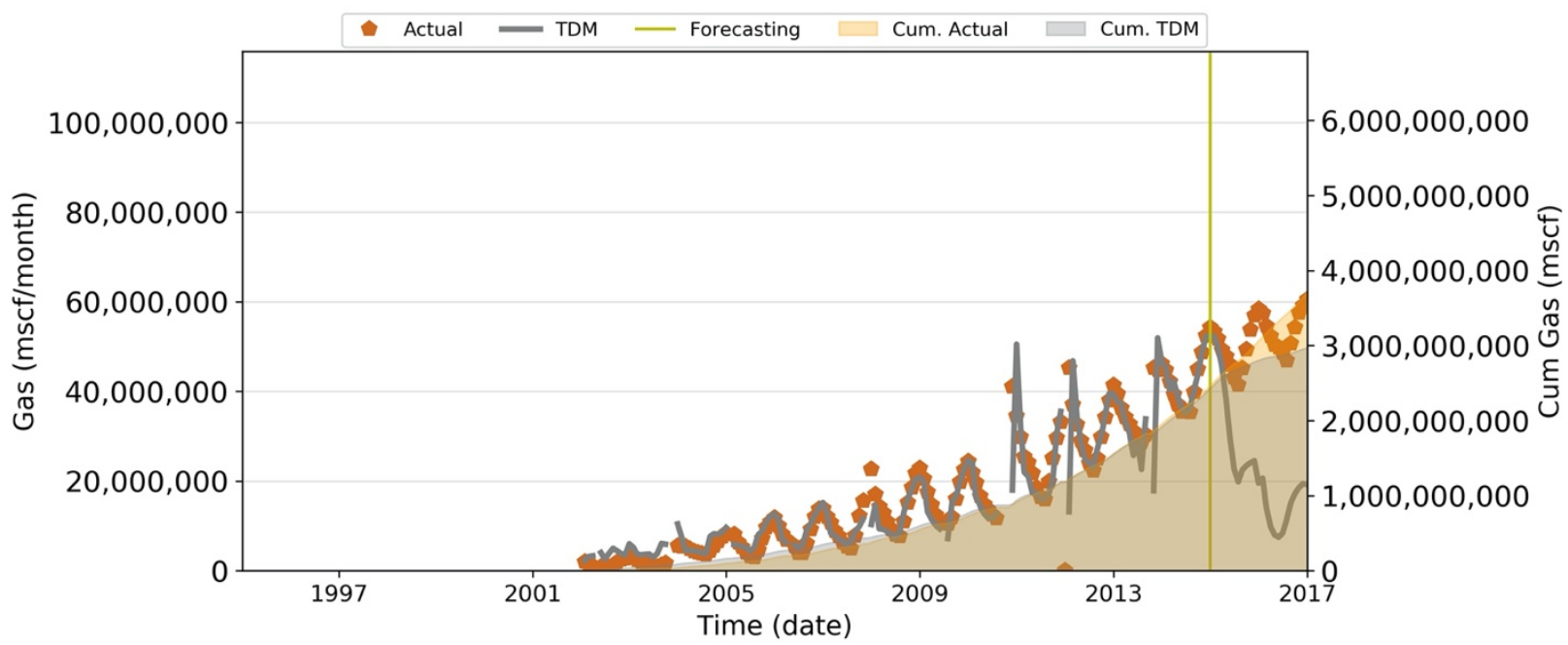


Gas: P-37

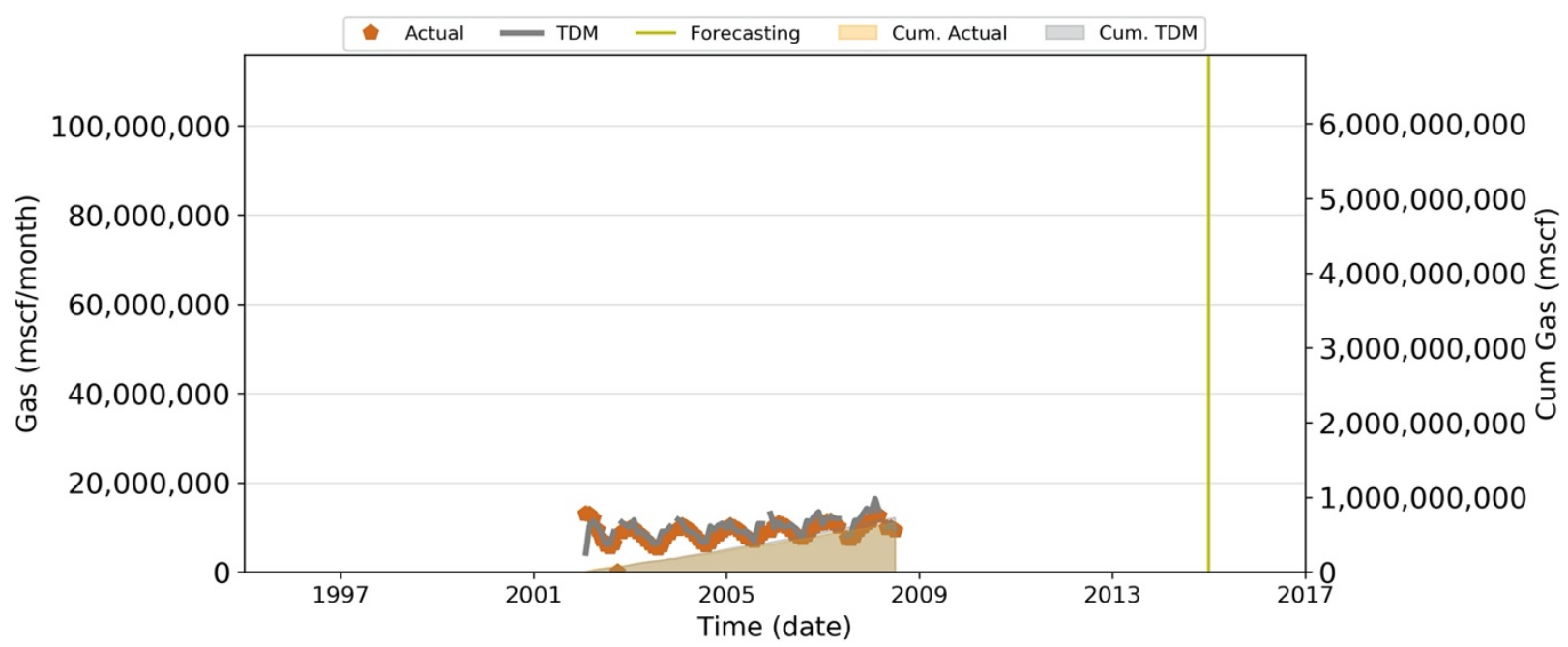

Gas: P-38

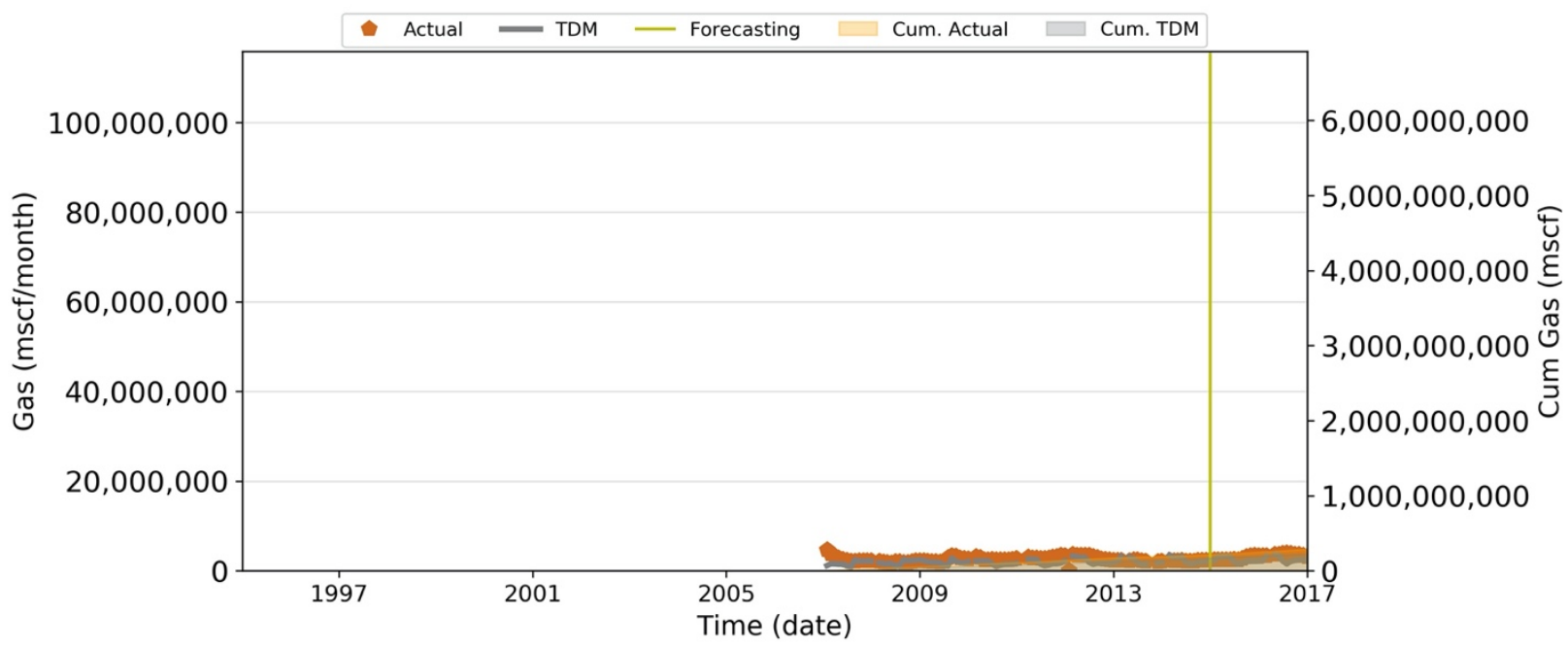

Gas: P-39

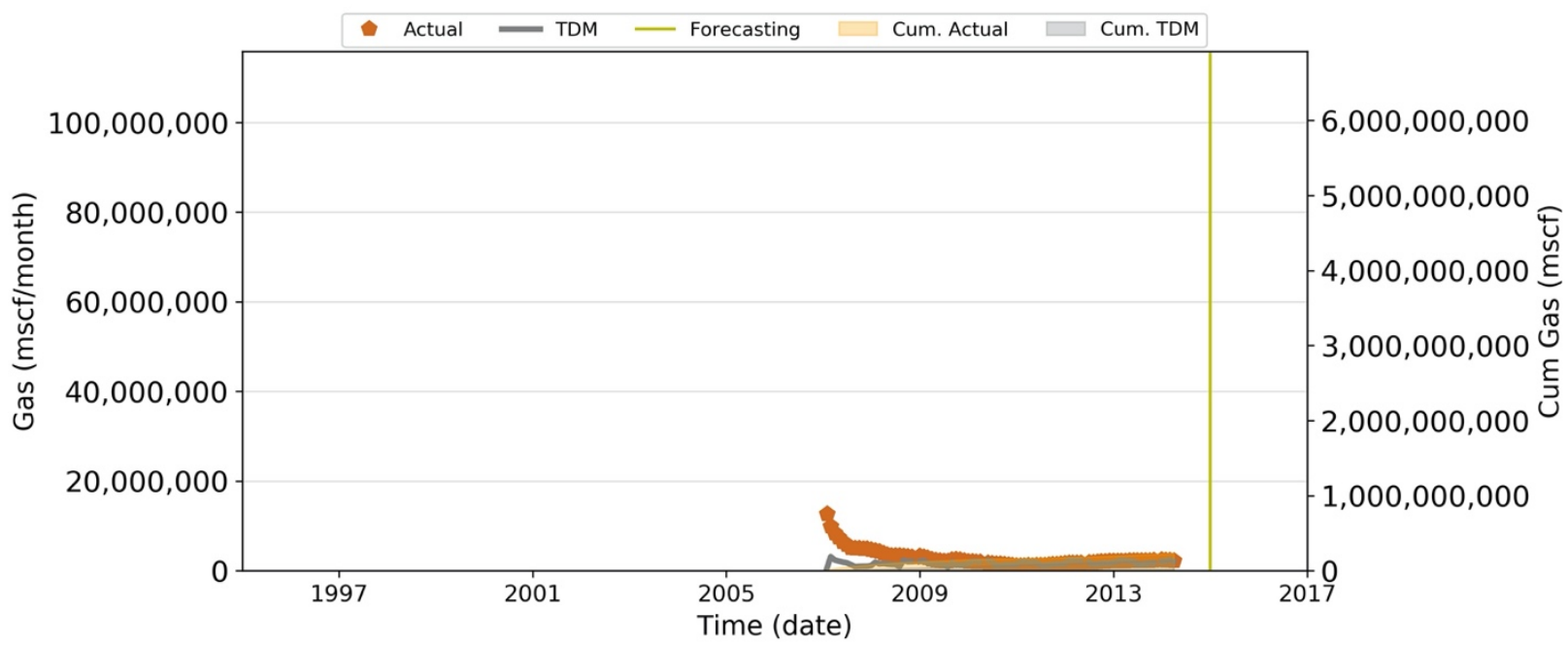


Gas: P-41

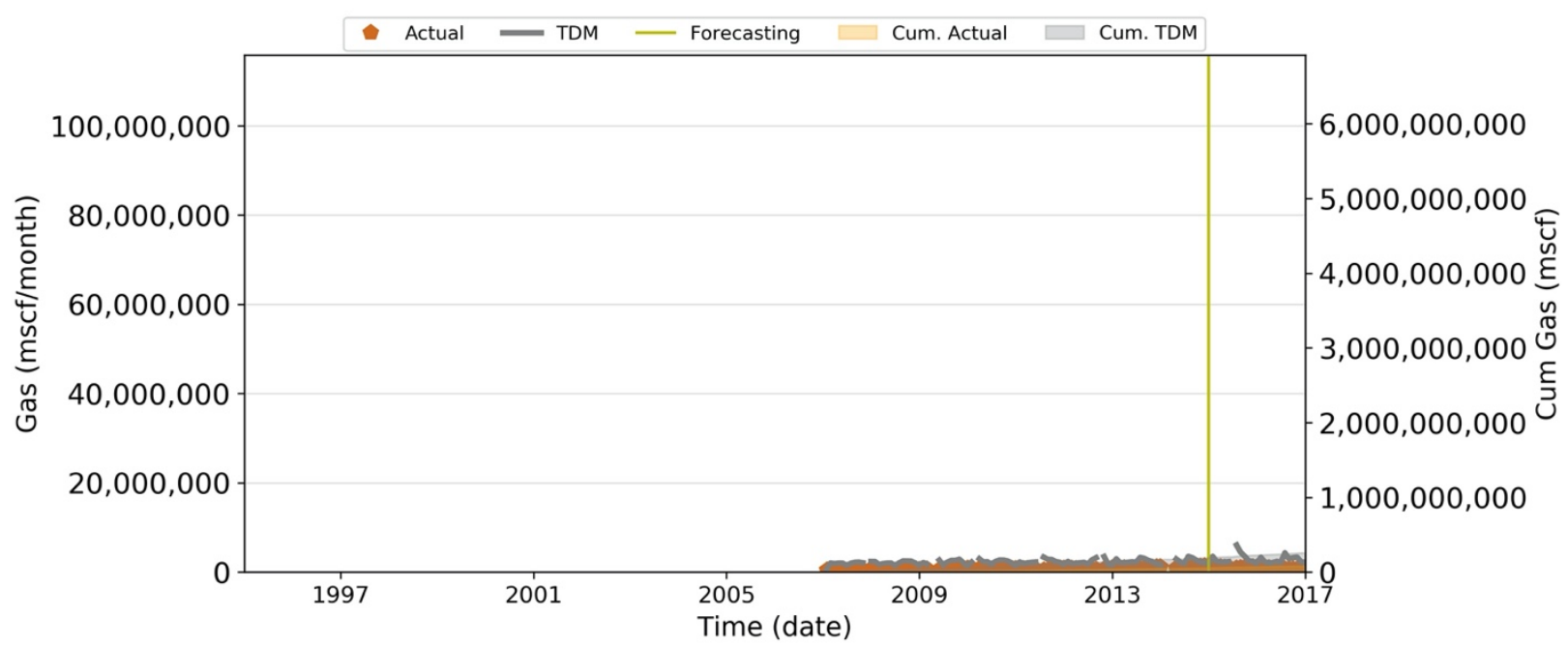

Gas: P-42

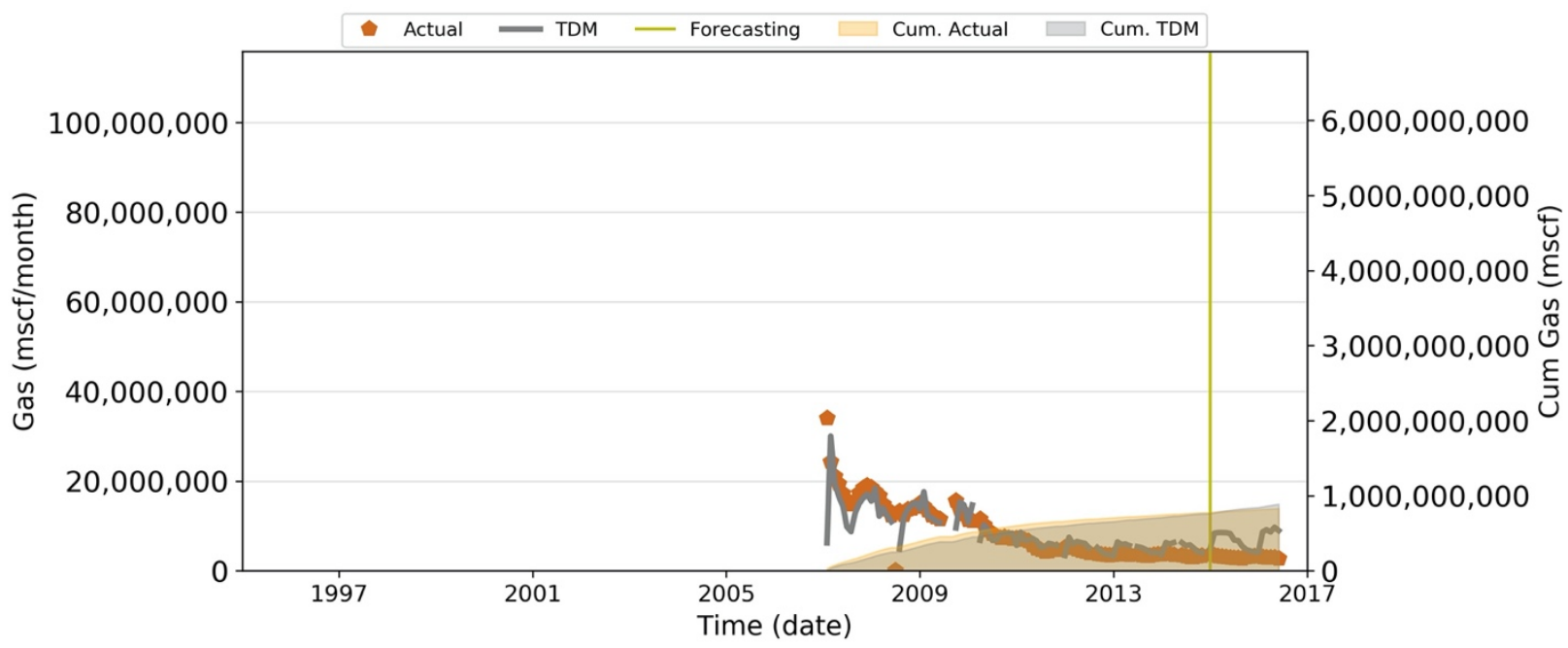

Gas: P-43

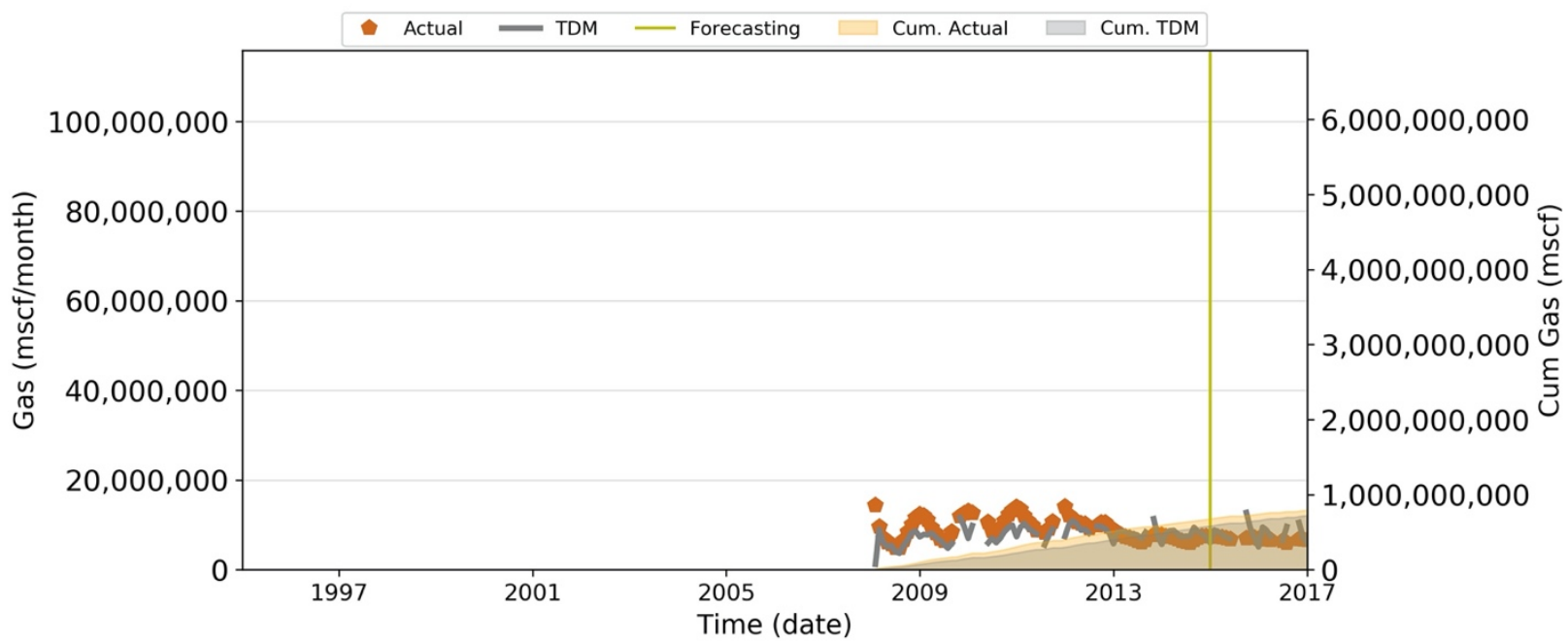


Gas: P-44

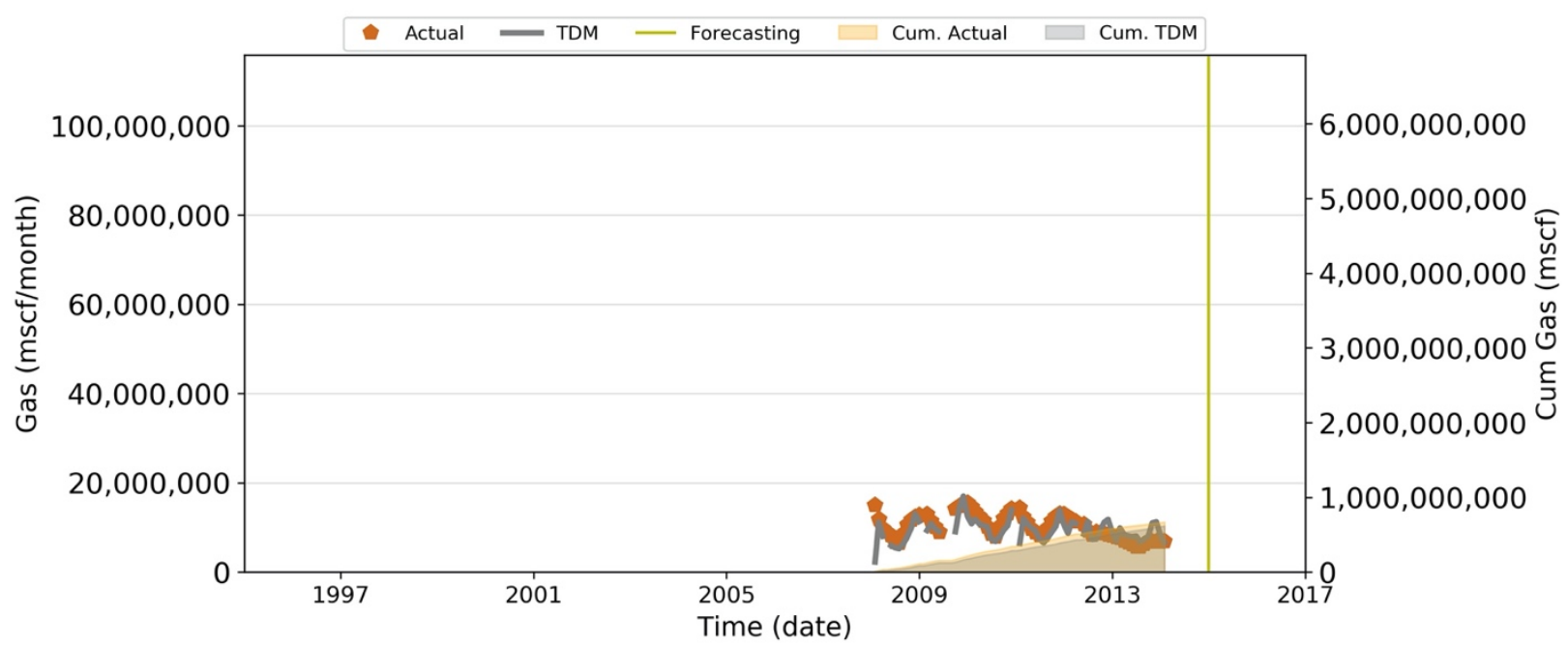

Gas: P-47

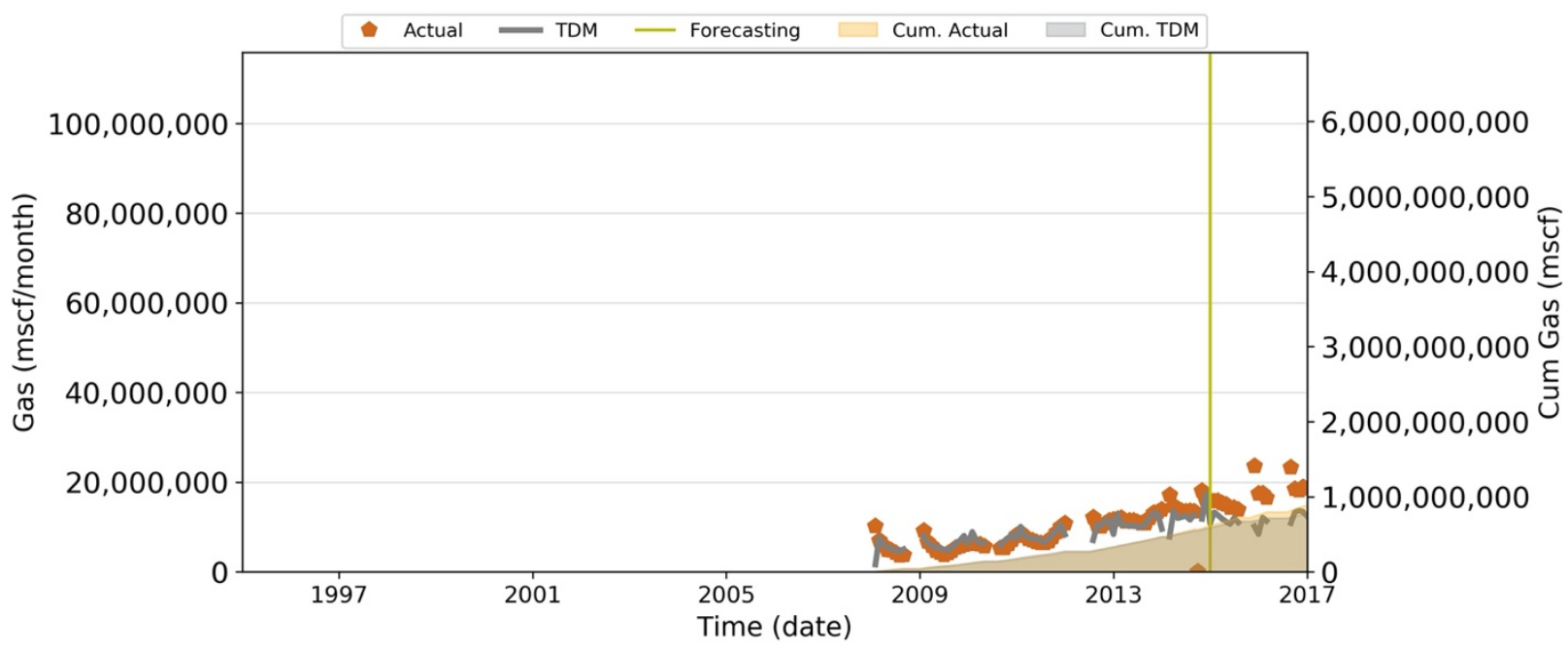

Gas: P-49

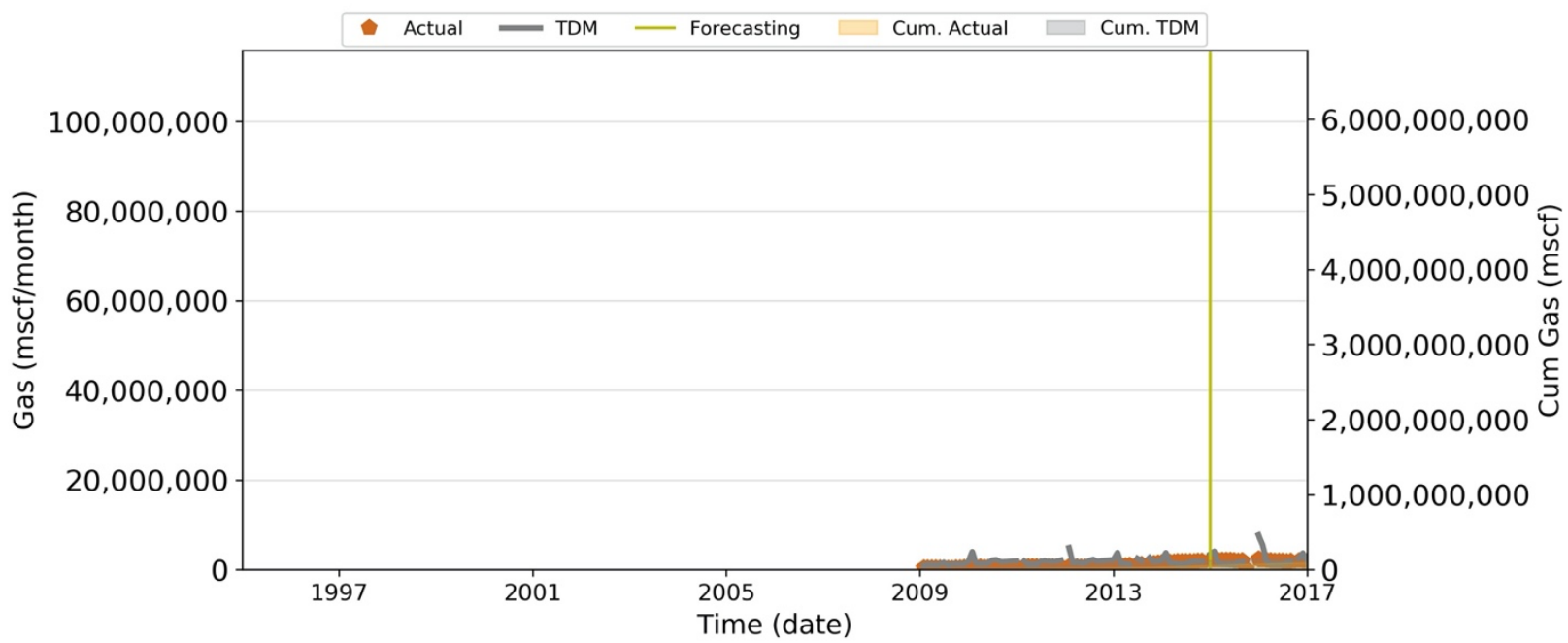


Gas: P-50

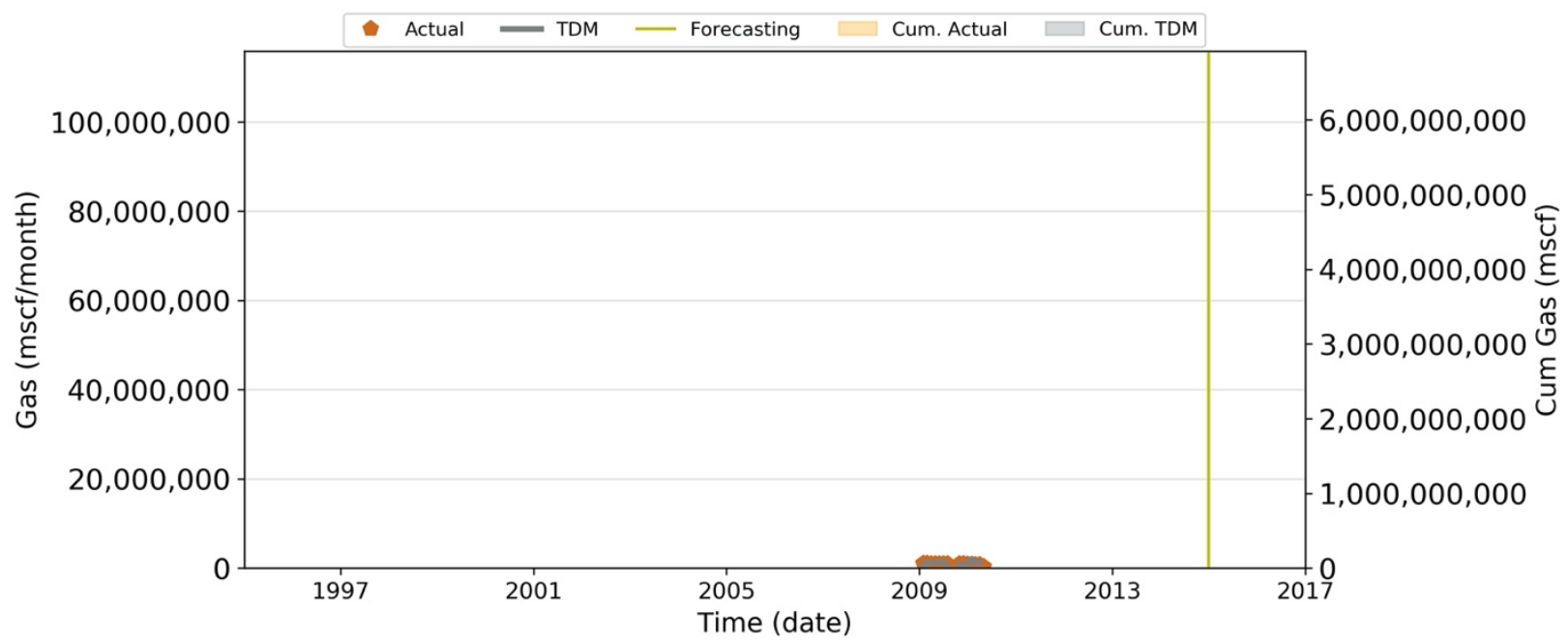

Gas: P-51

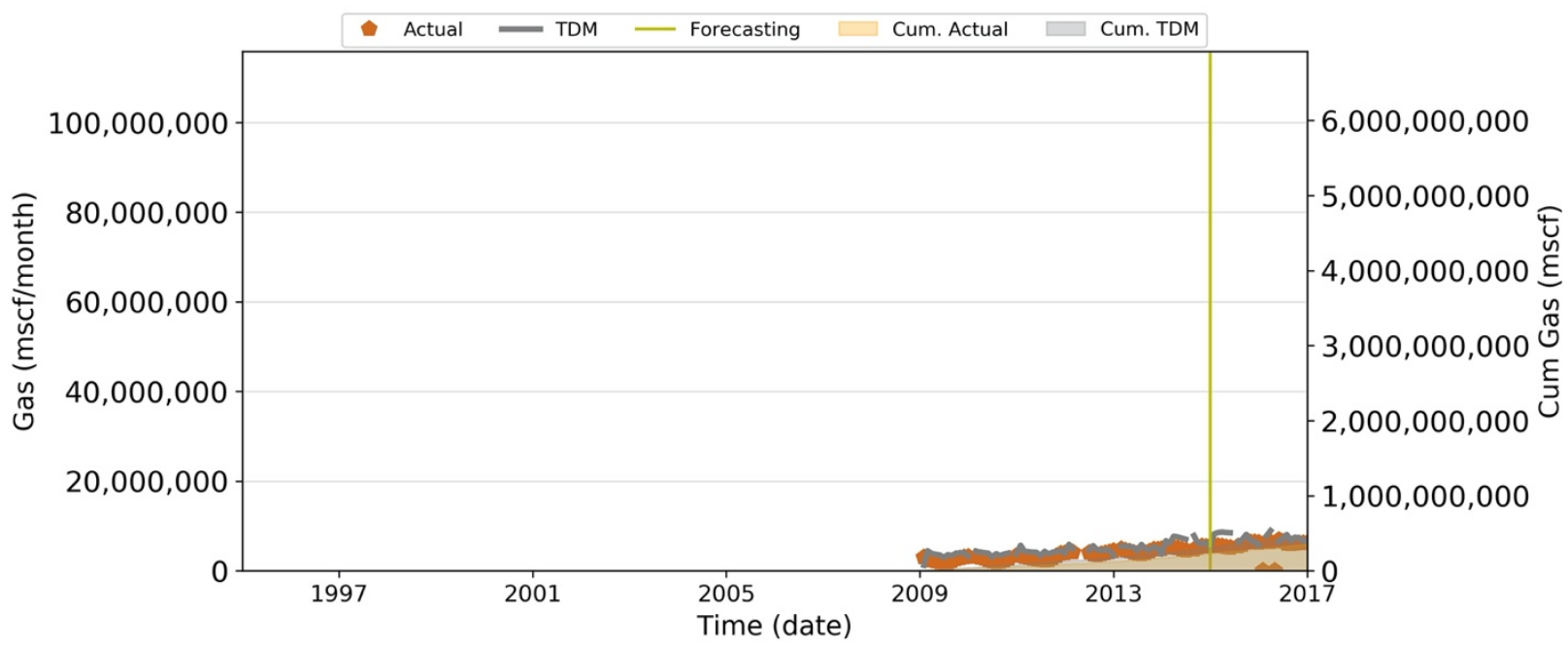

Gas: P-52

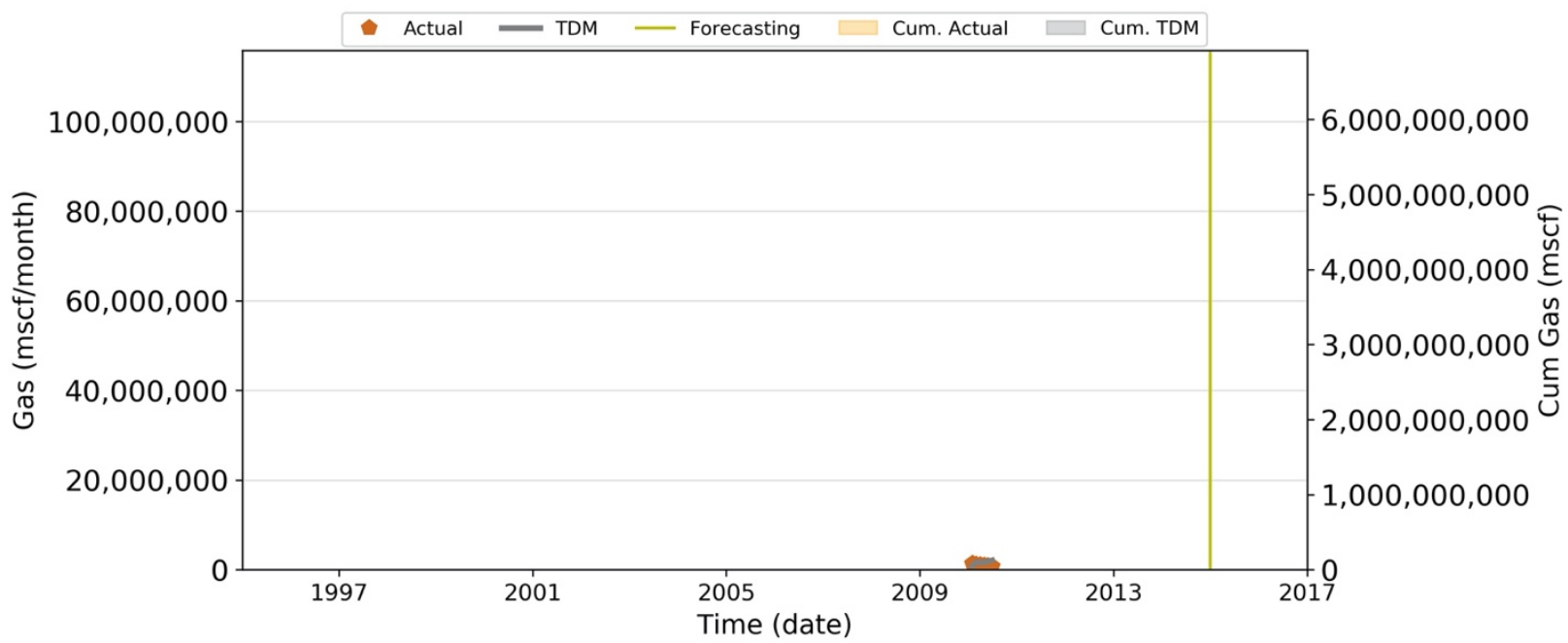


Gas: P-53

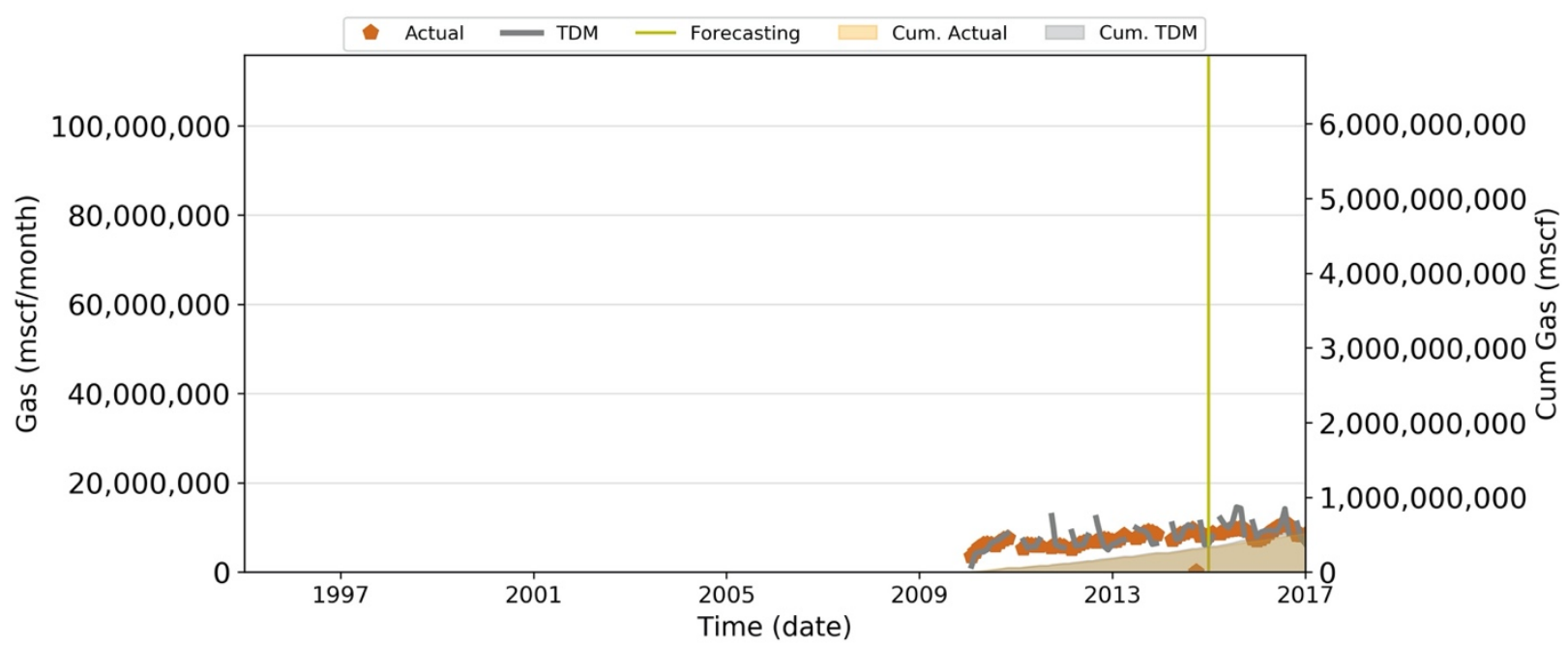

Gas: P-54

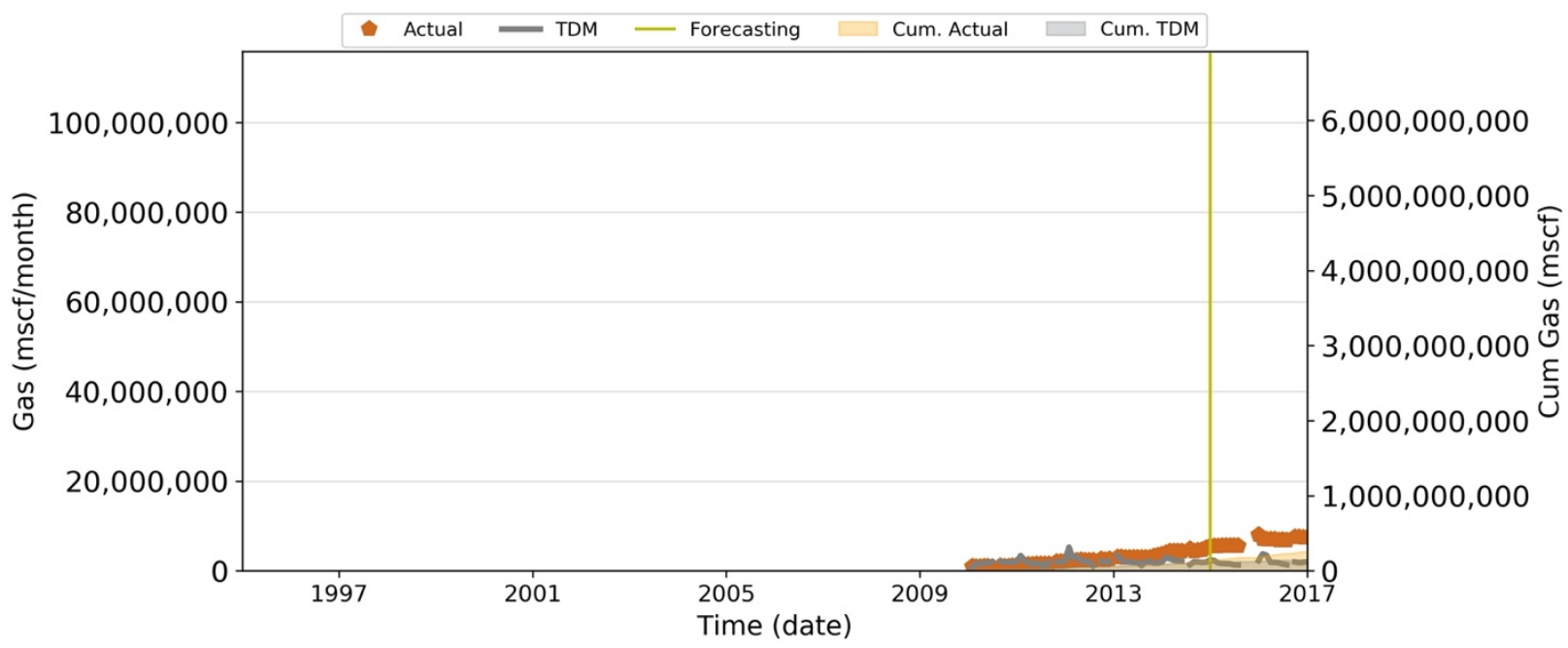

Gas: P-55

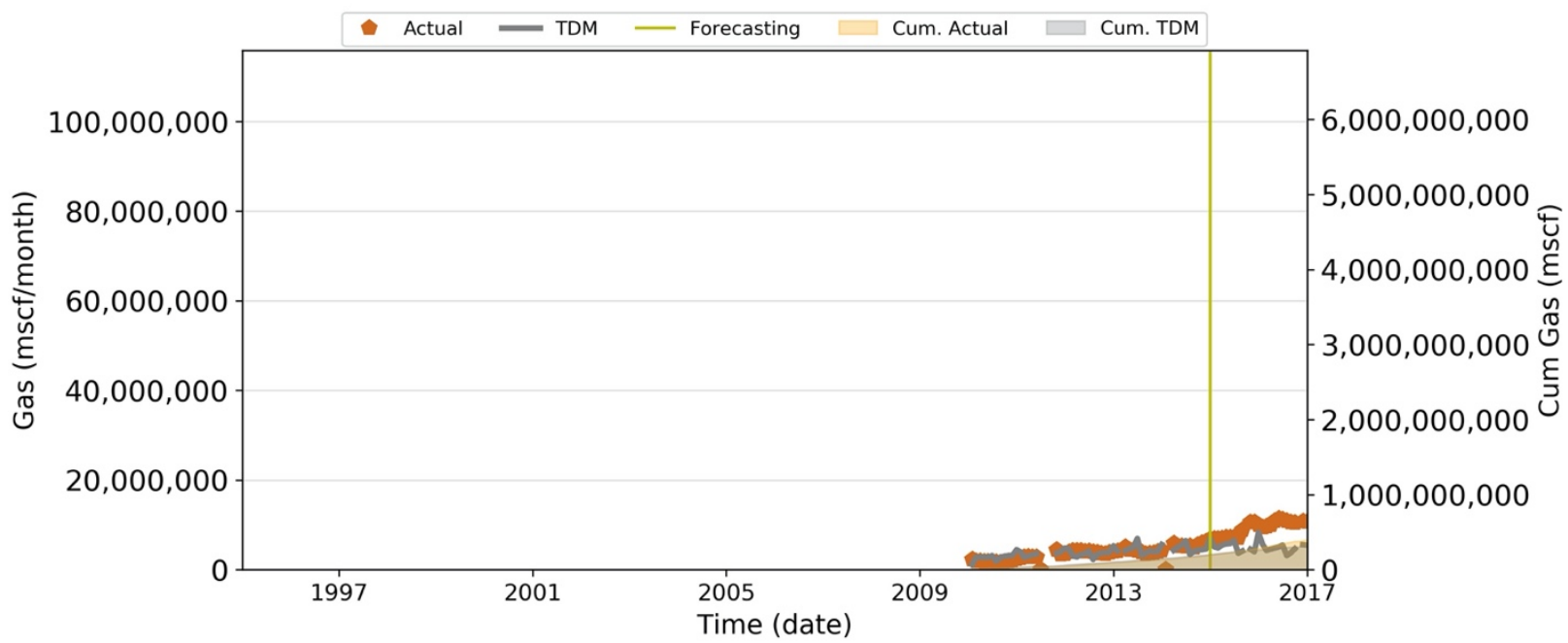


Gas: P-56

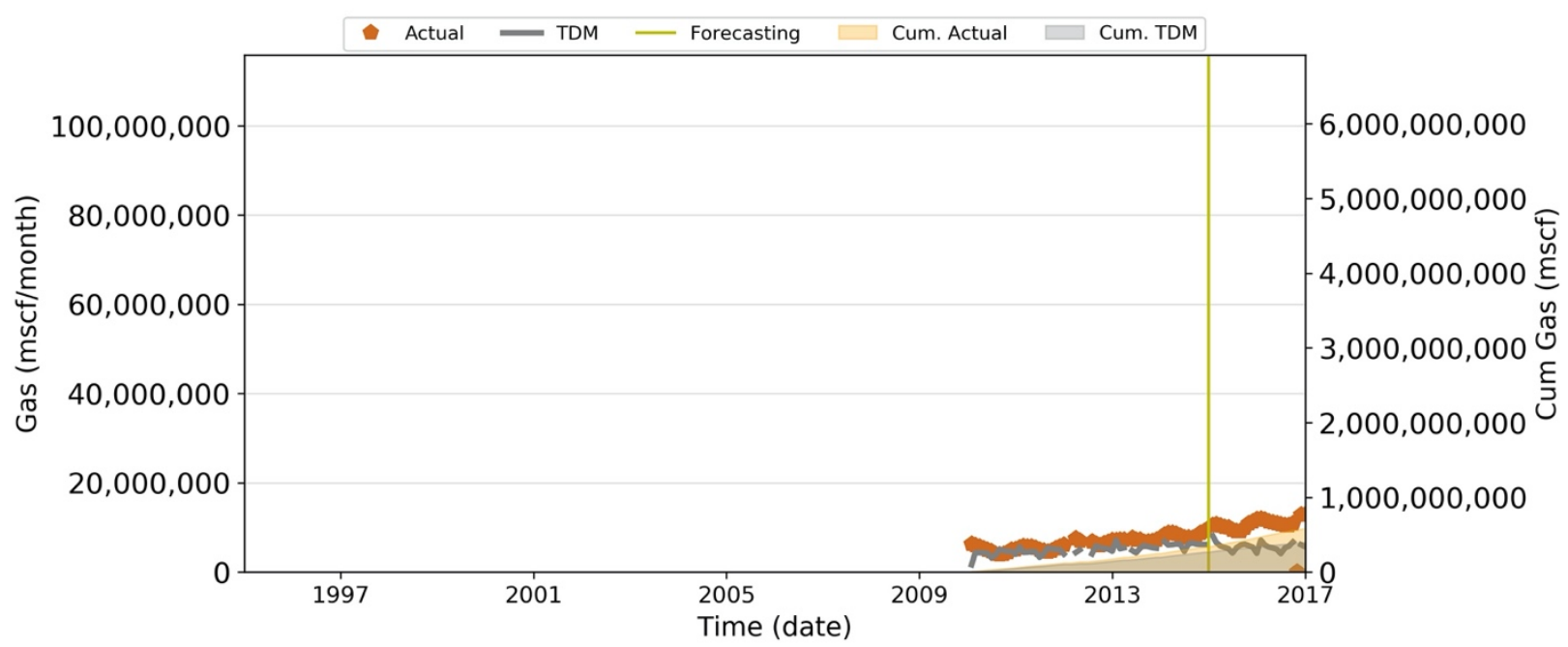

Gas: P-57

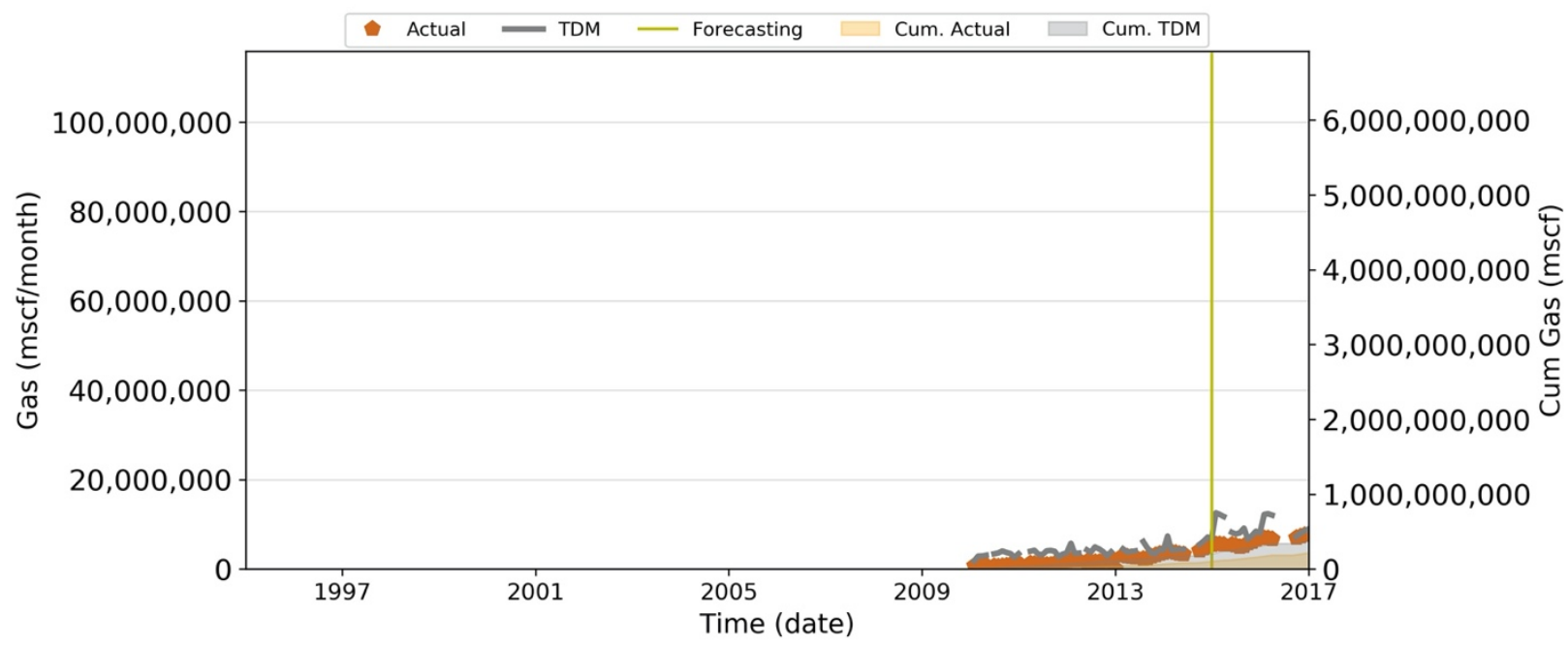


Water Results

Water: P-01

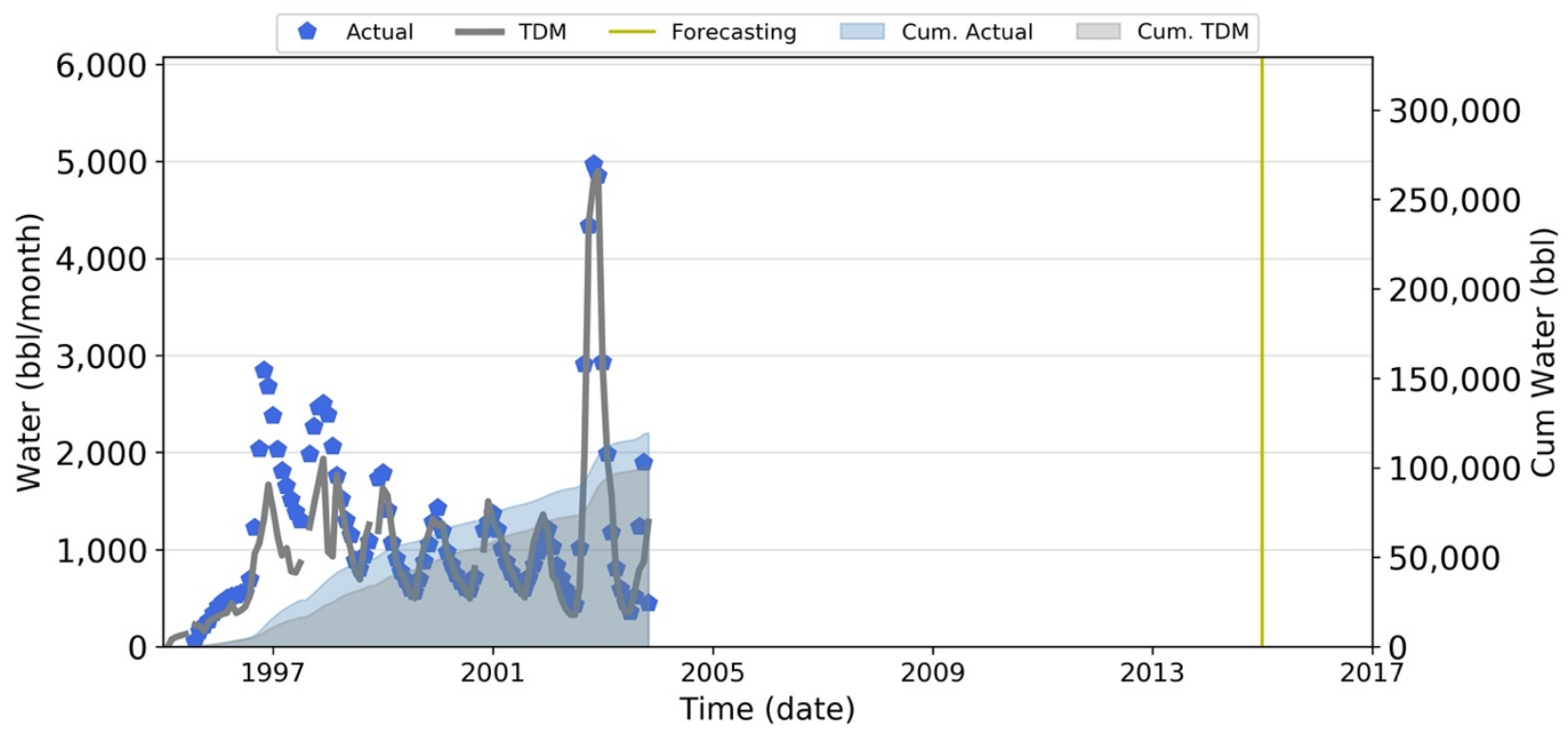

Water: P-02

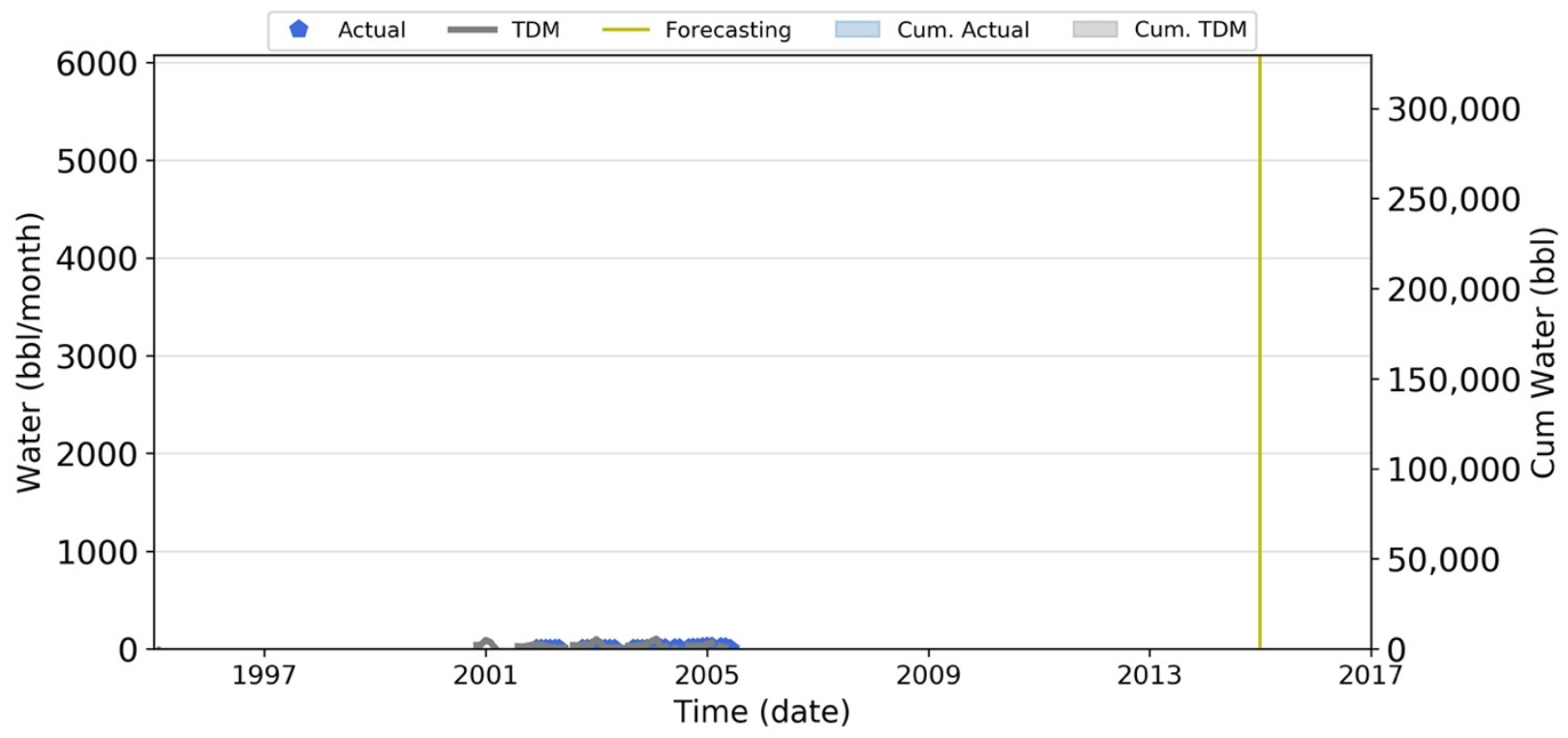


Water: P-03

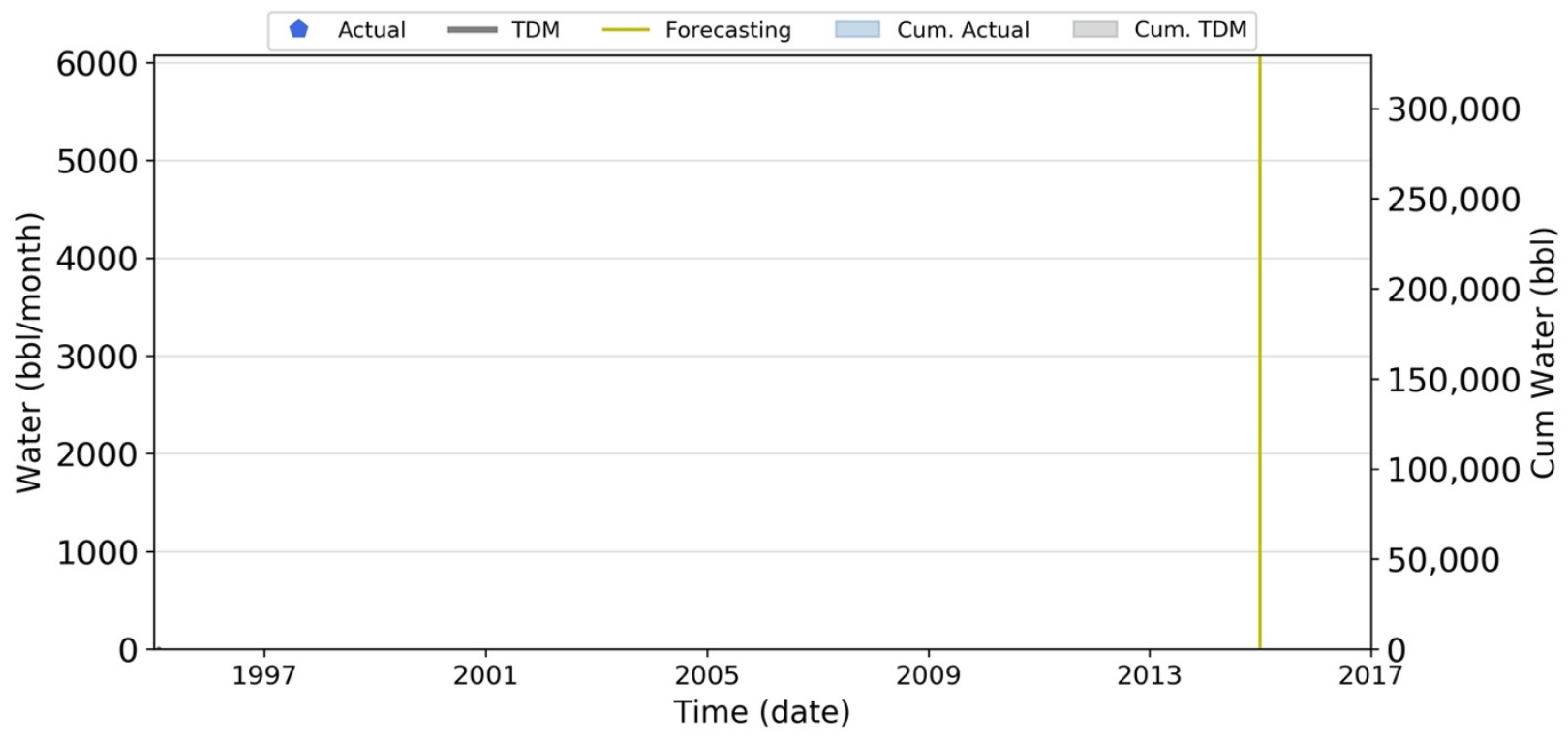

Water: P-04

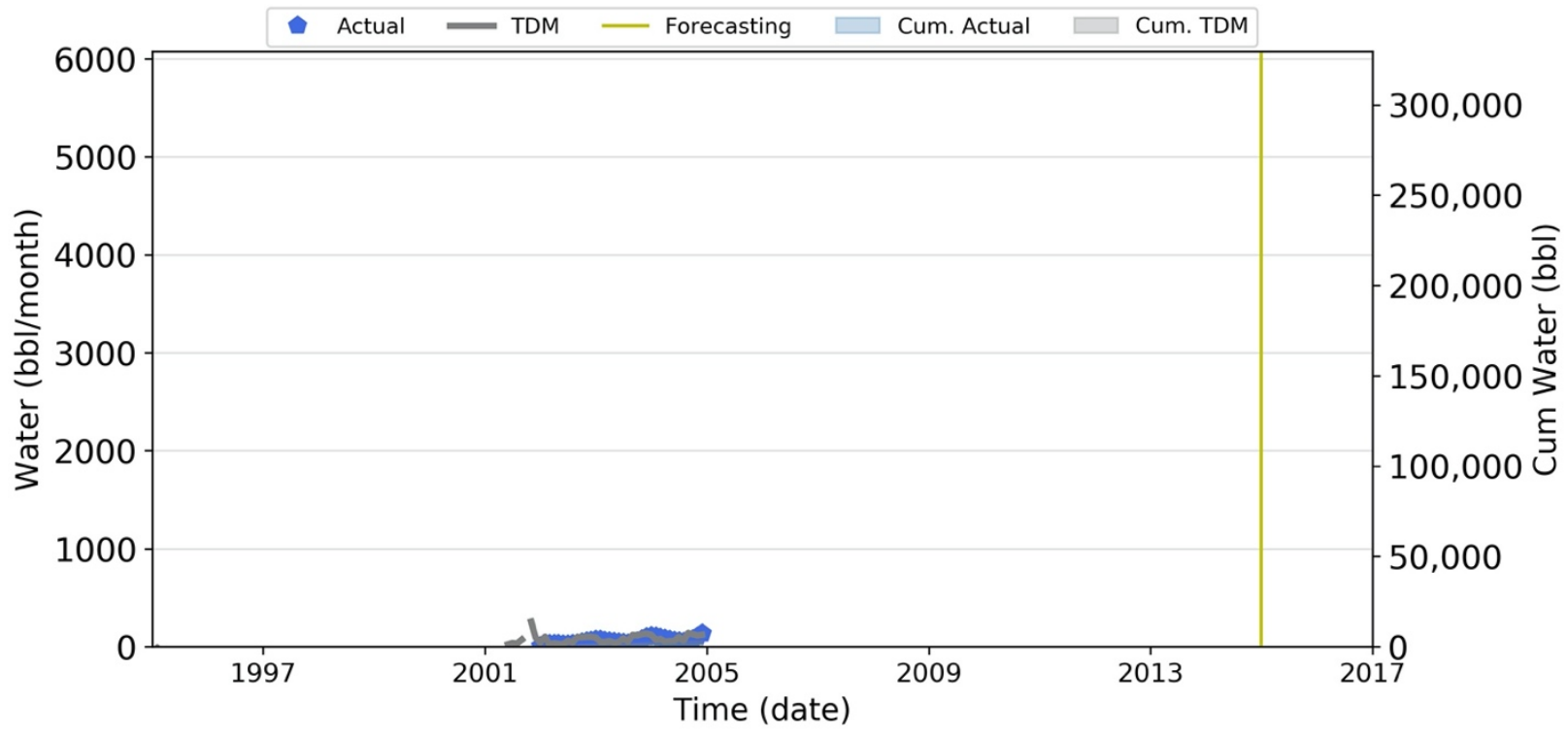


Water: P-05
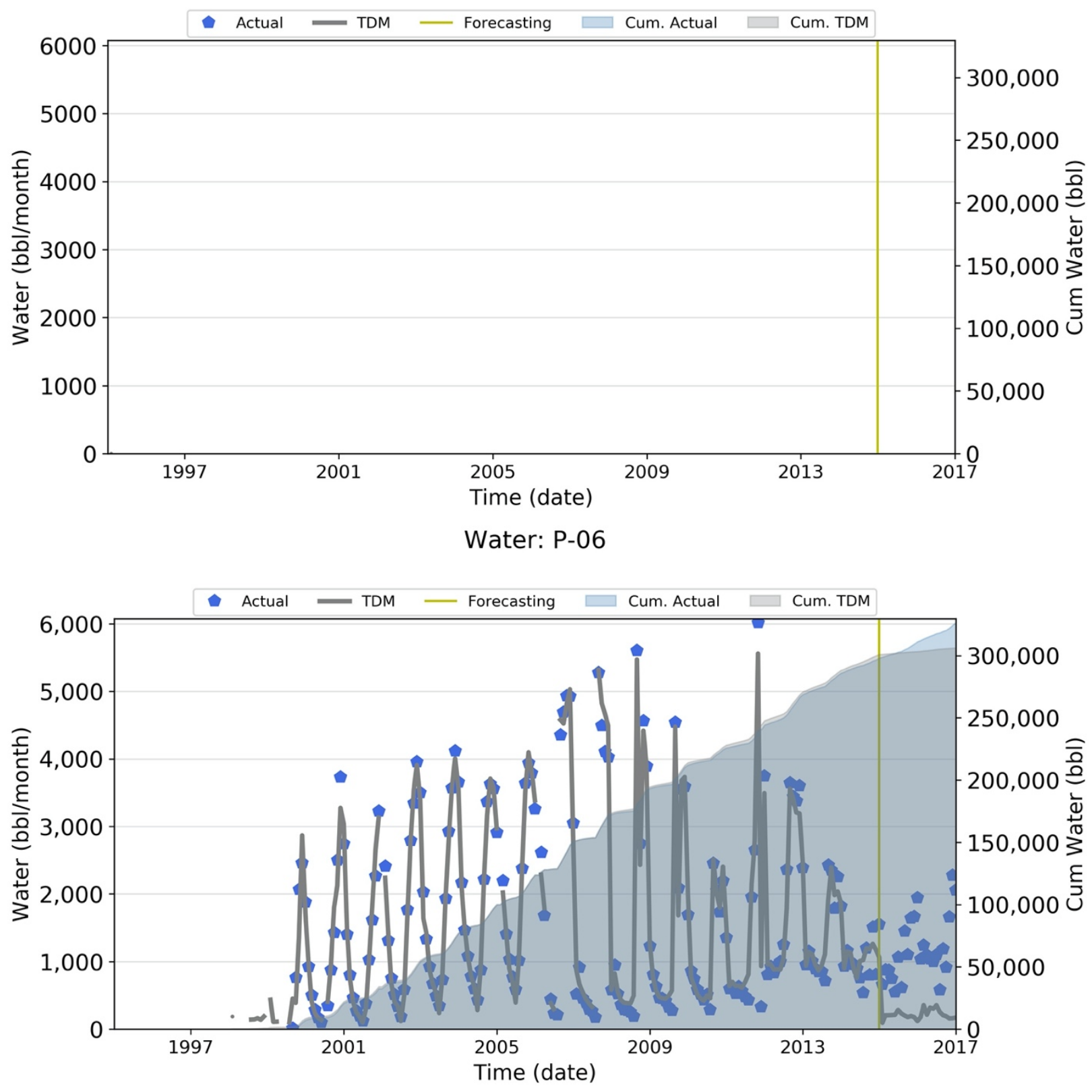


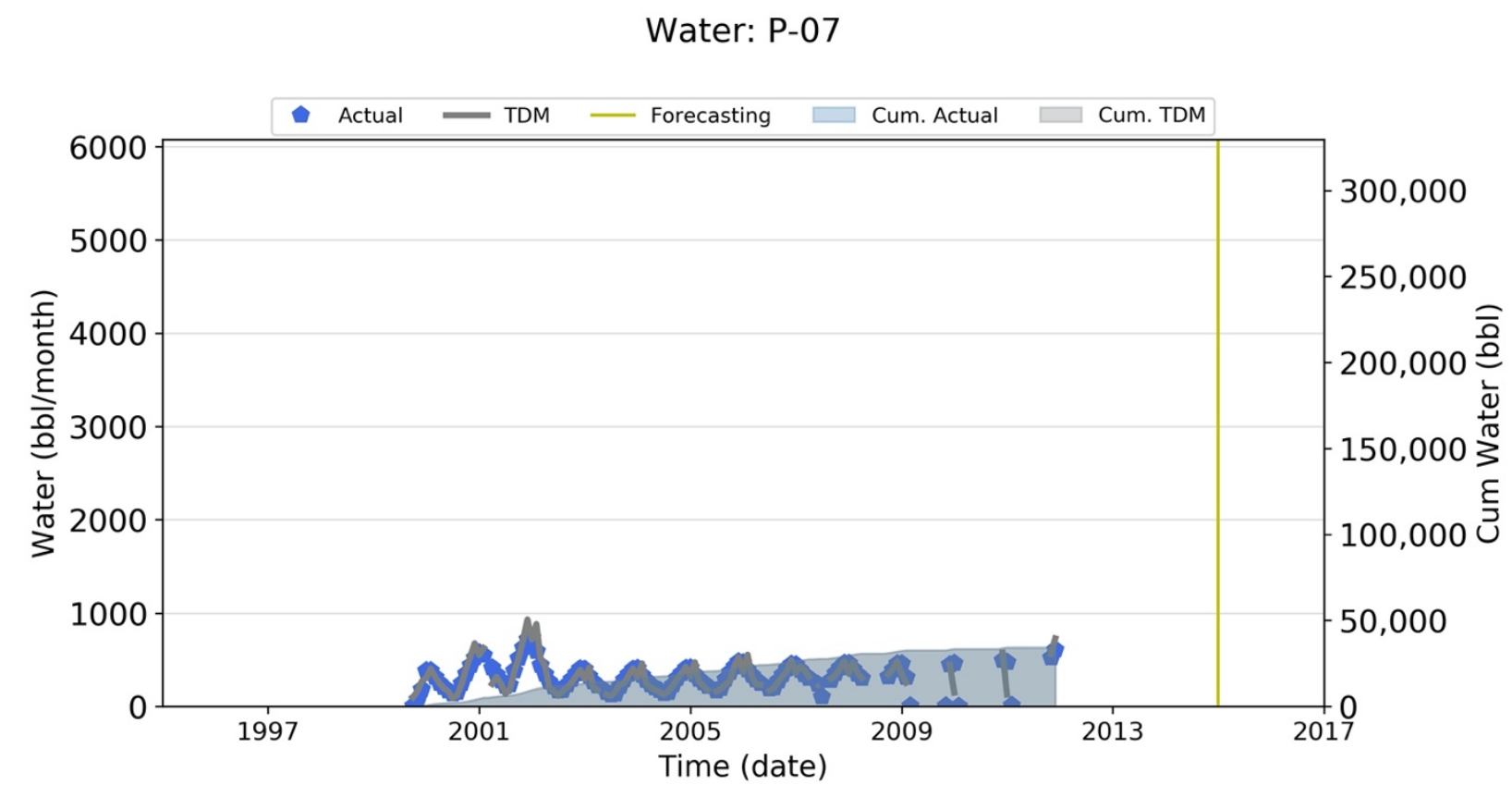

Water: P-09

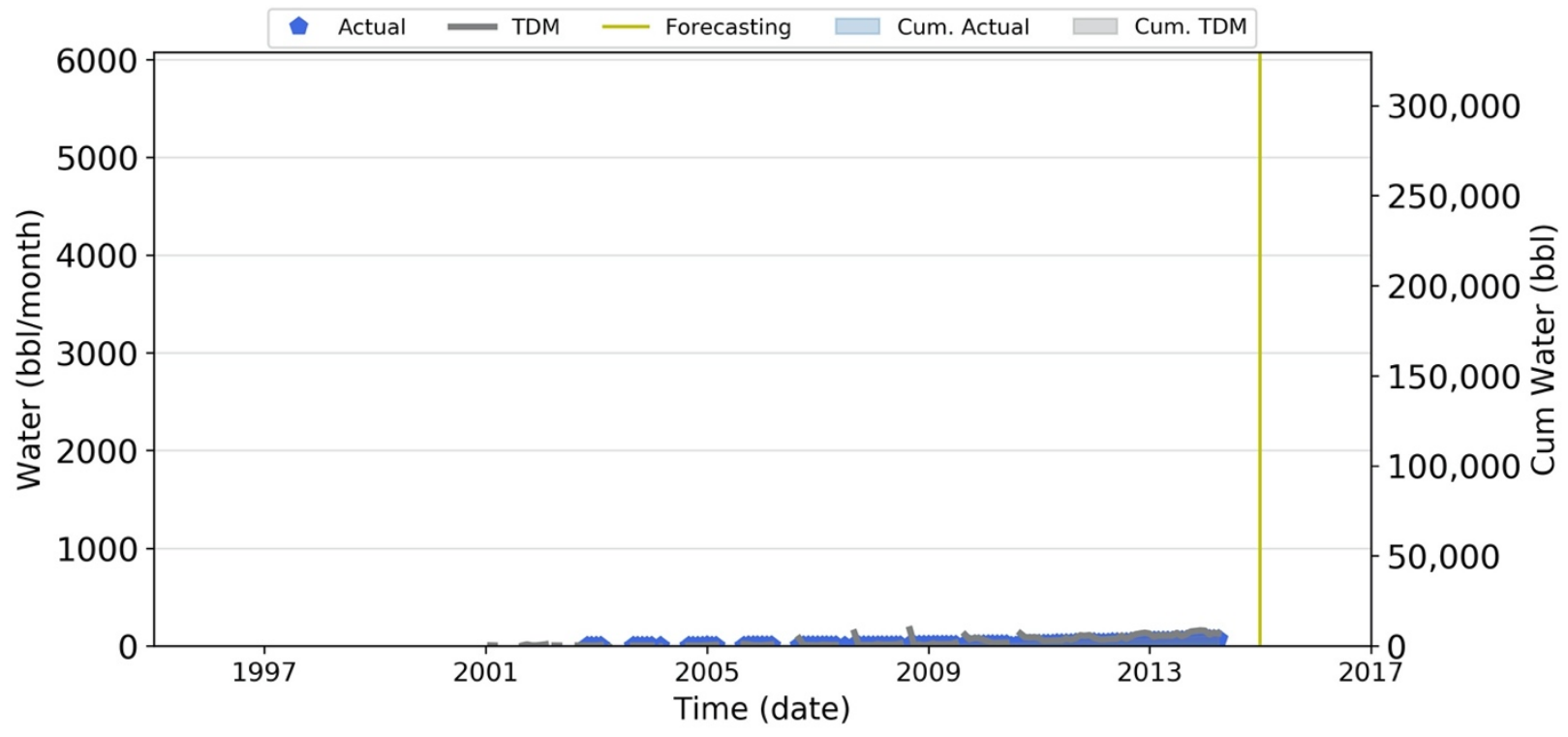


Water: P-10

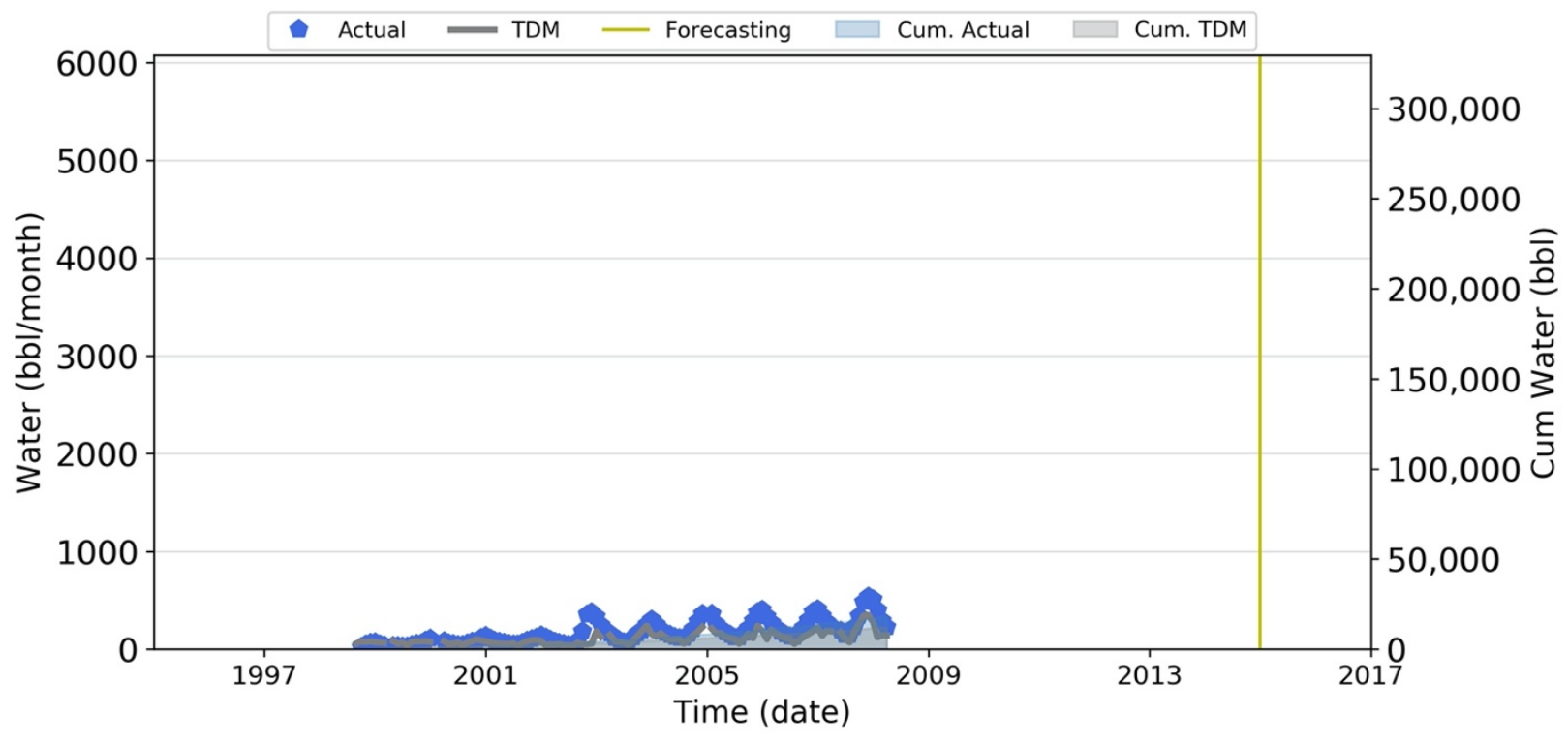

Water: P-11

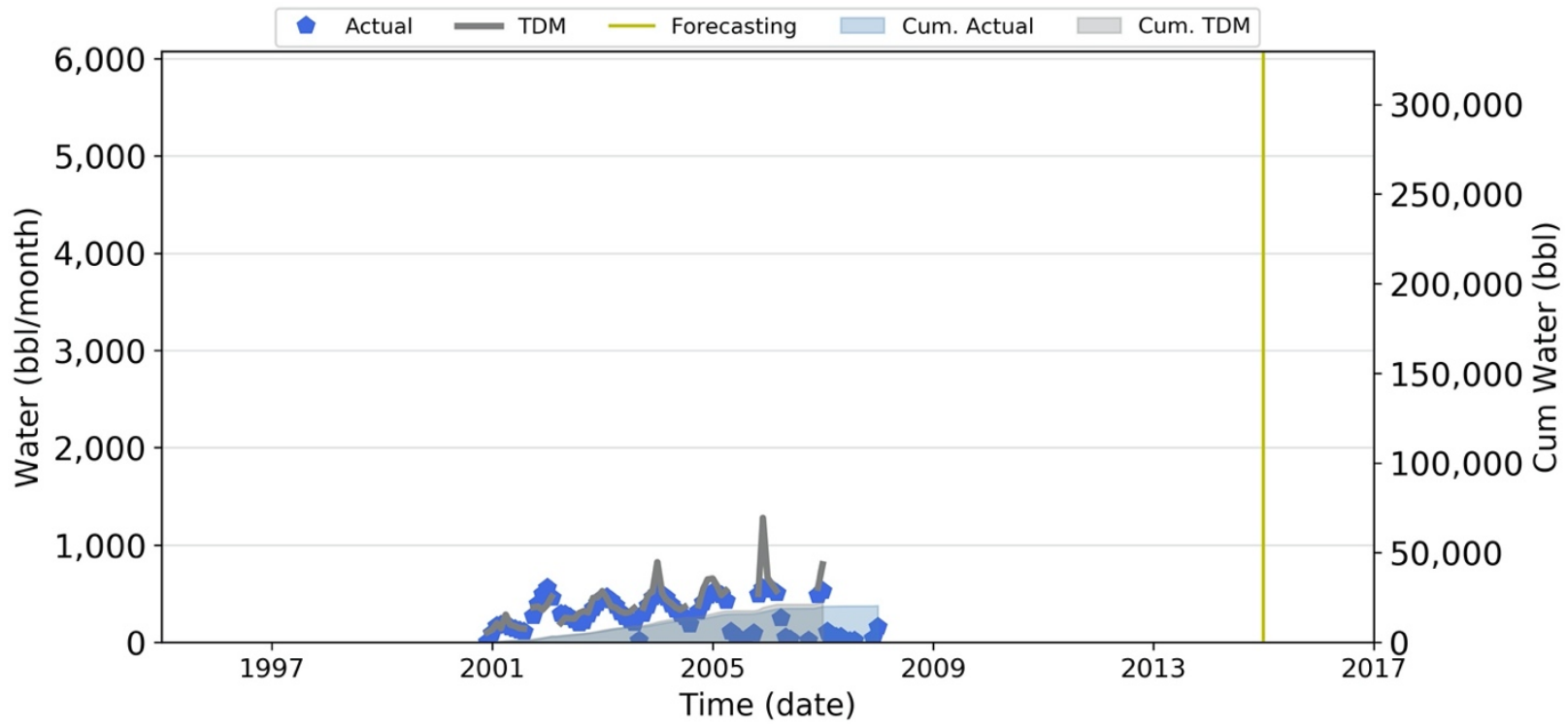


Water: P-13

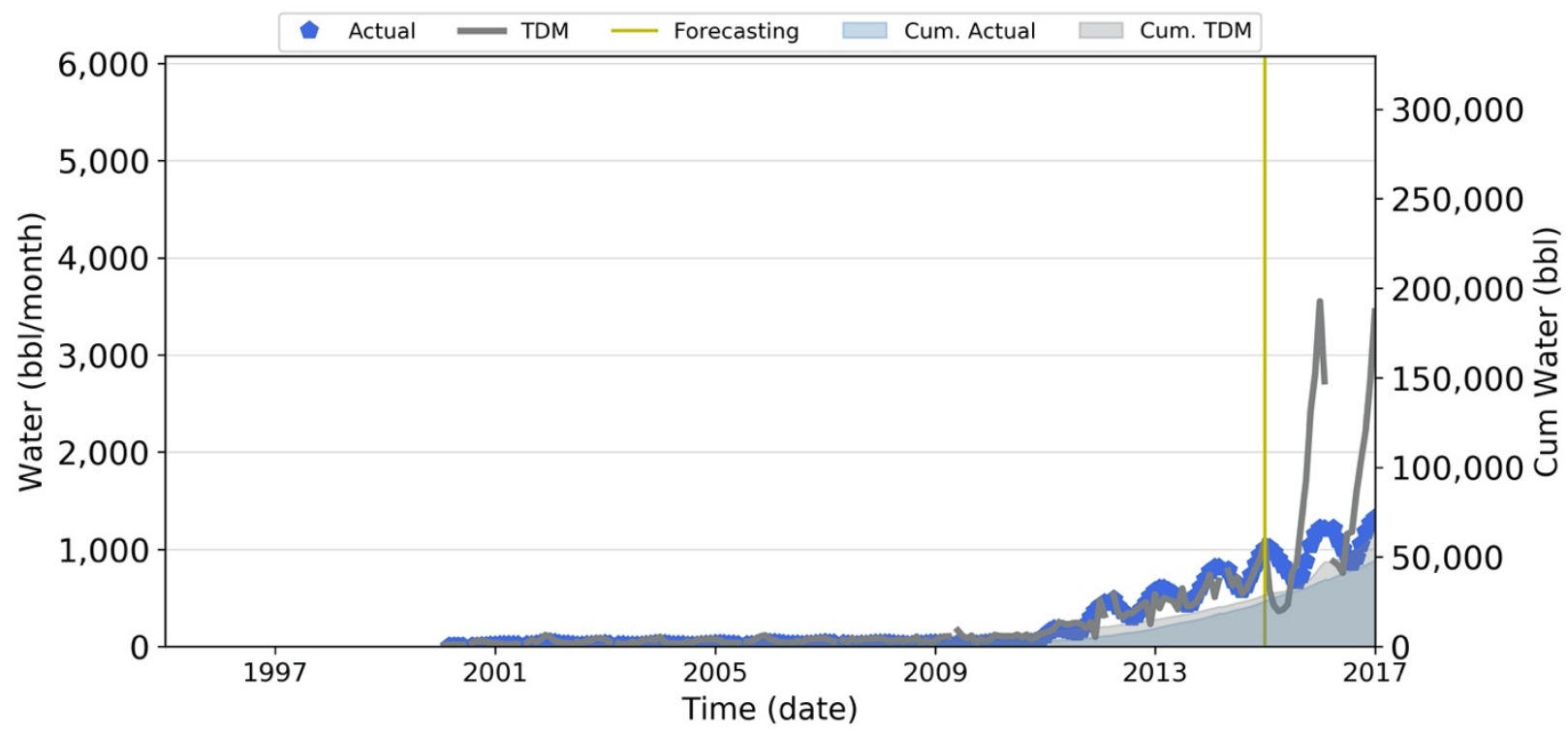

Water: P-14

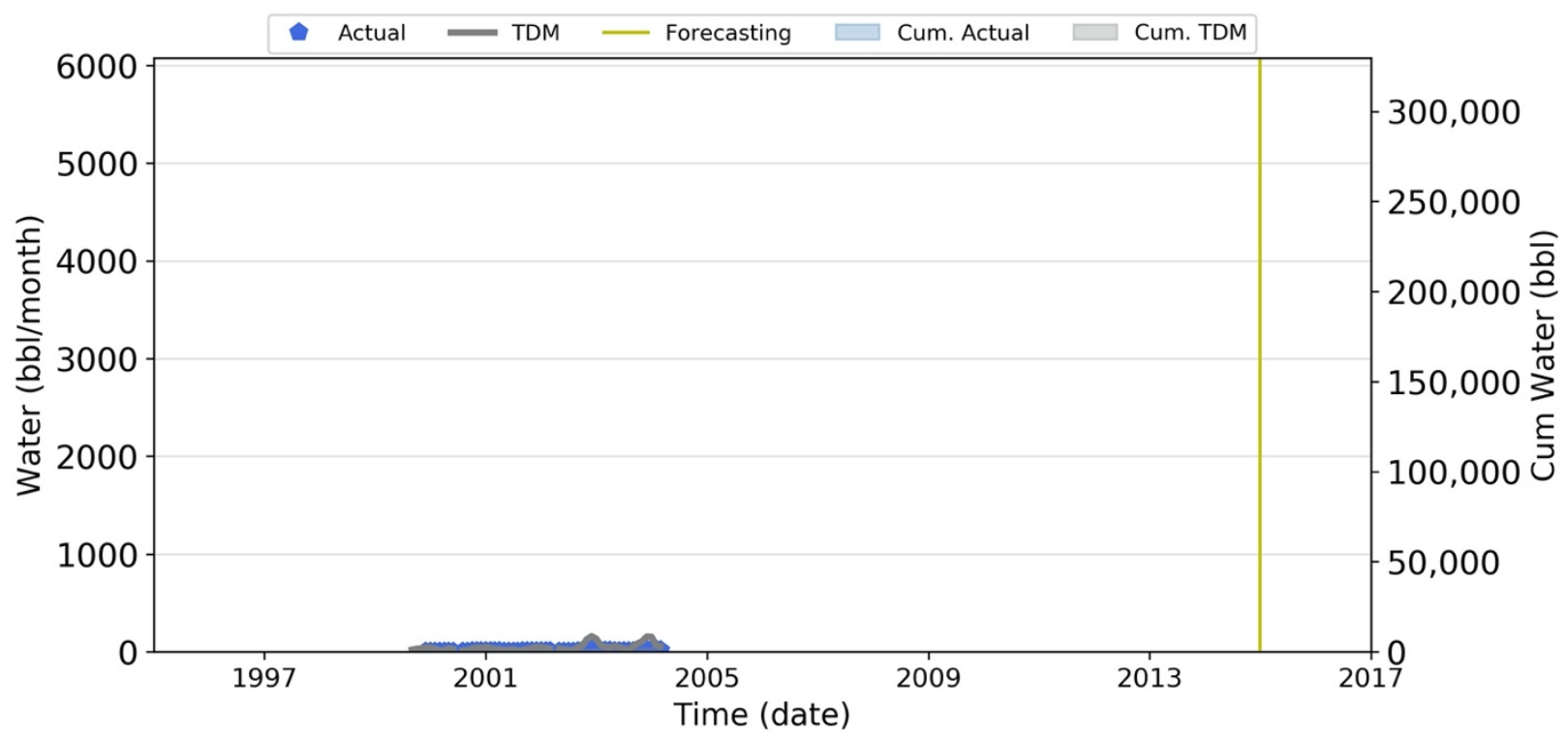


Water: P-15

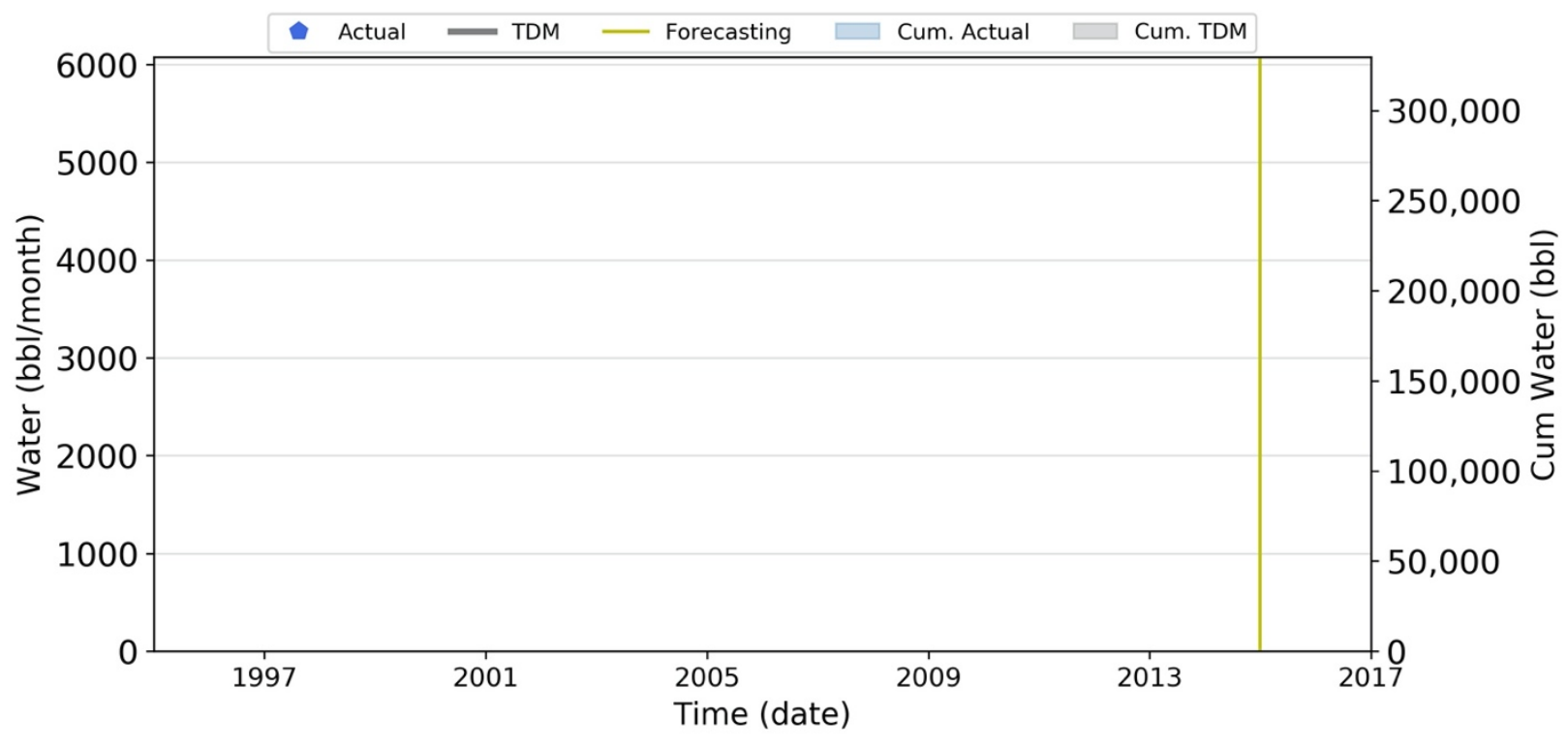

Water: P-16

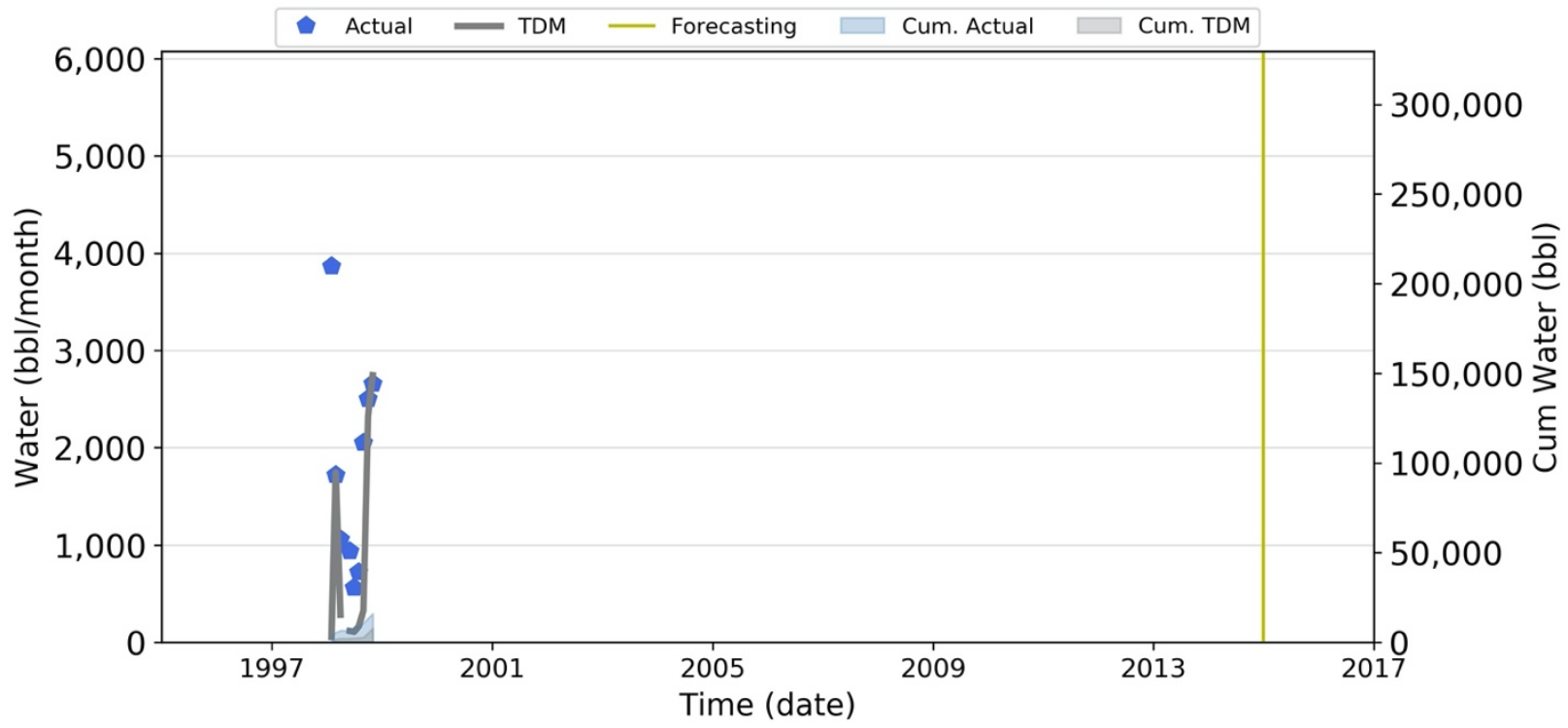


Water: P-17

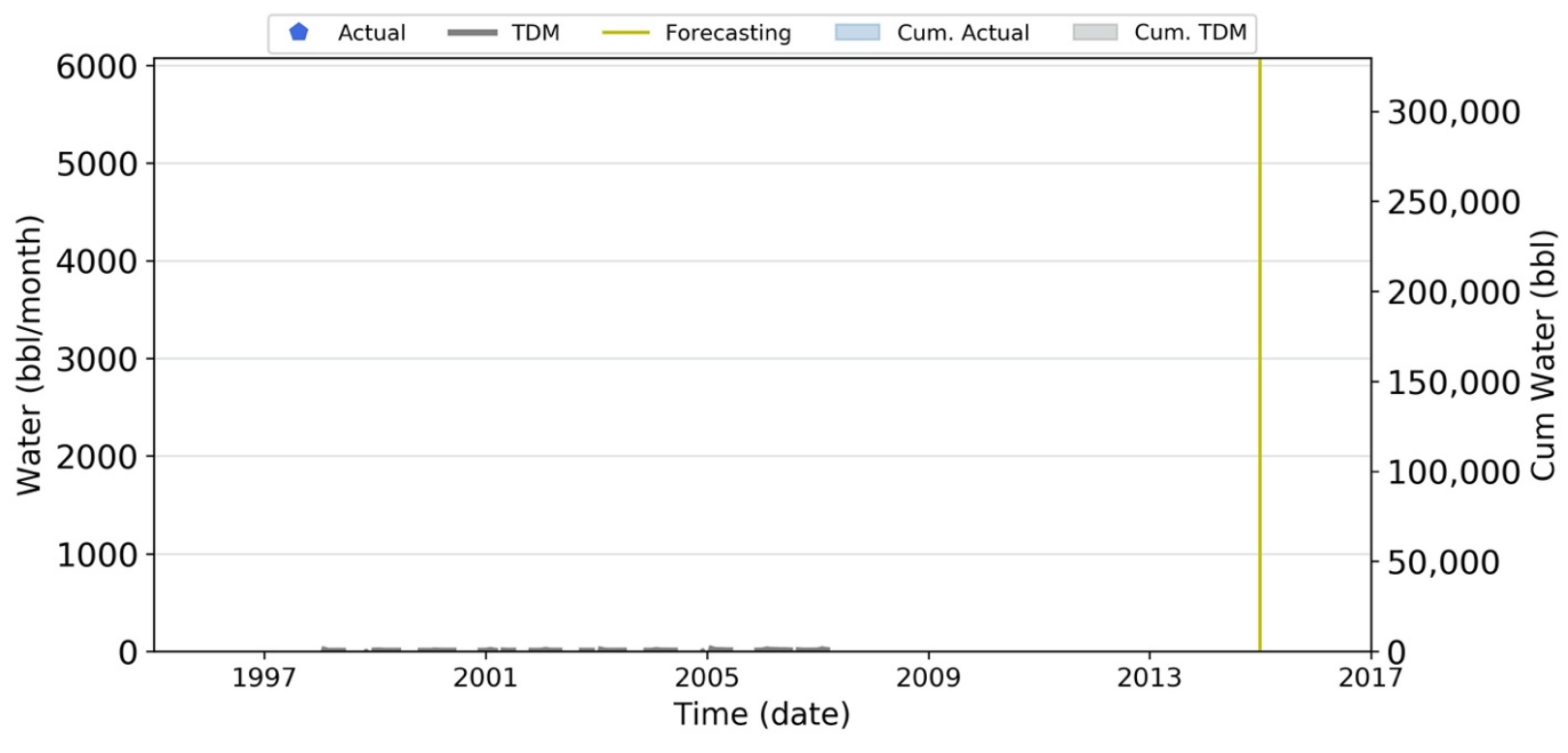

Water: P-18

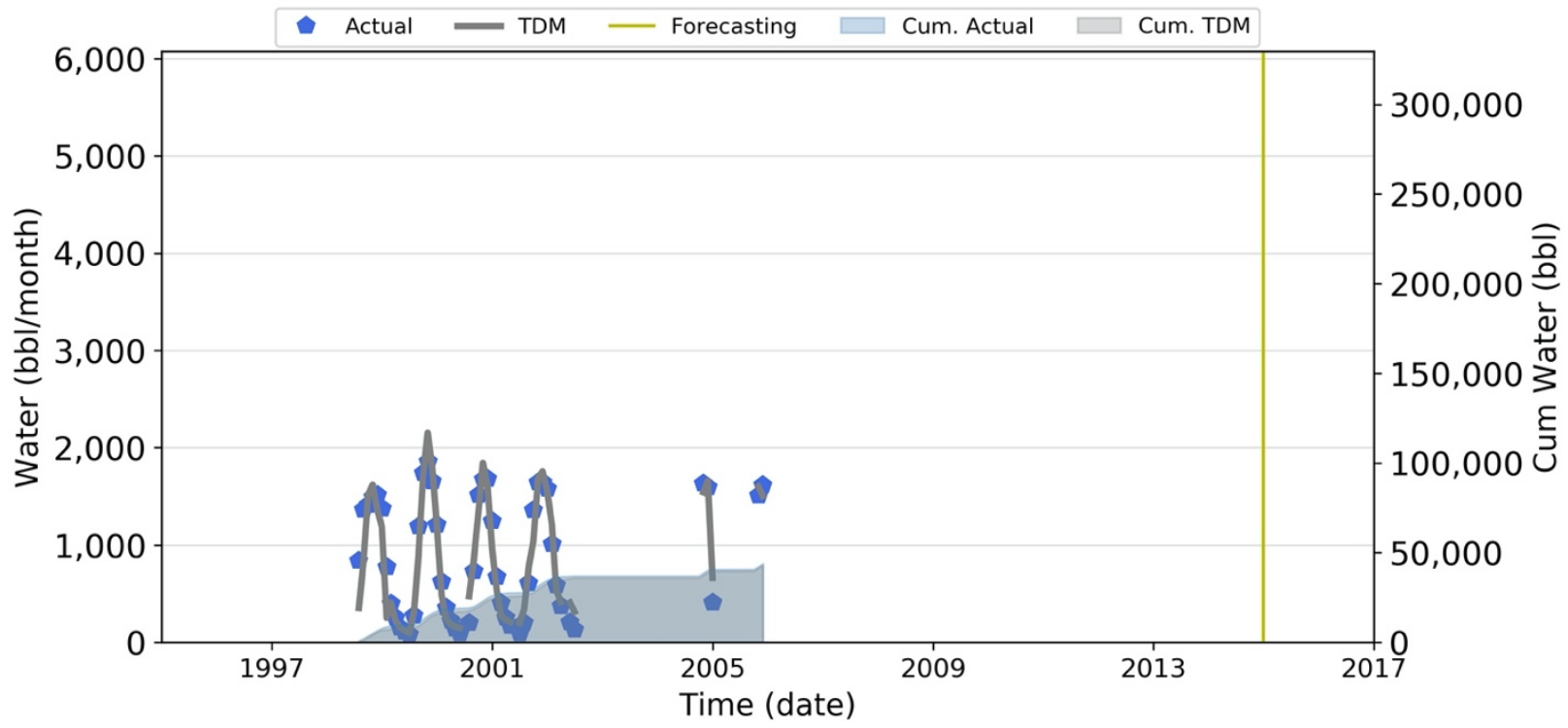


Water: P-19

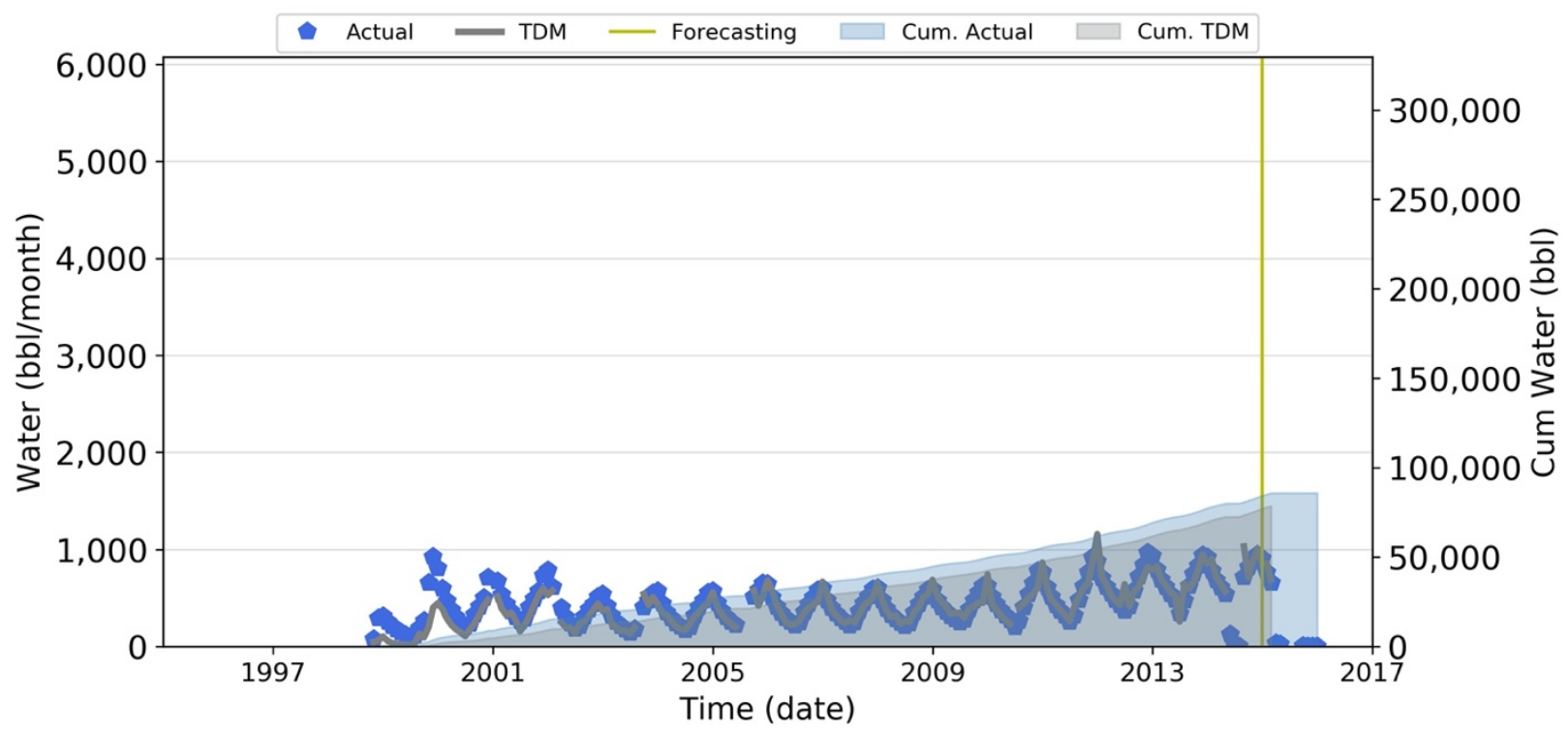

Water: P-20

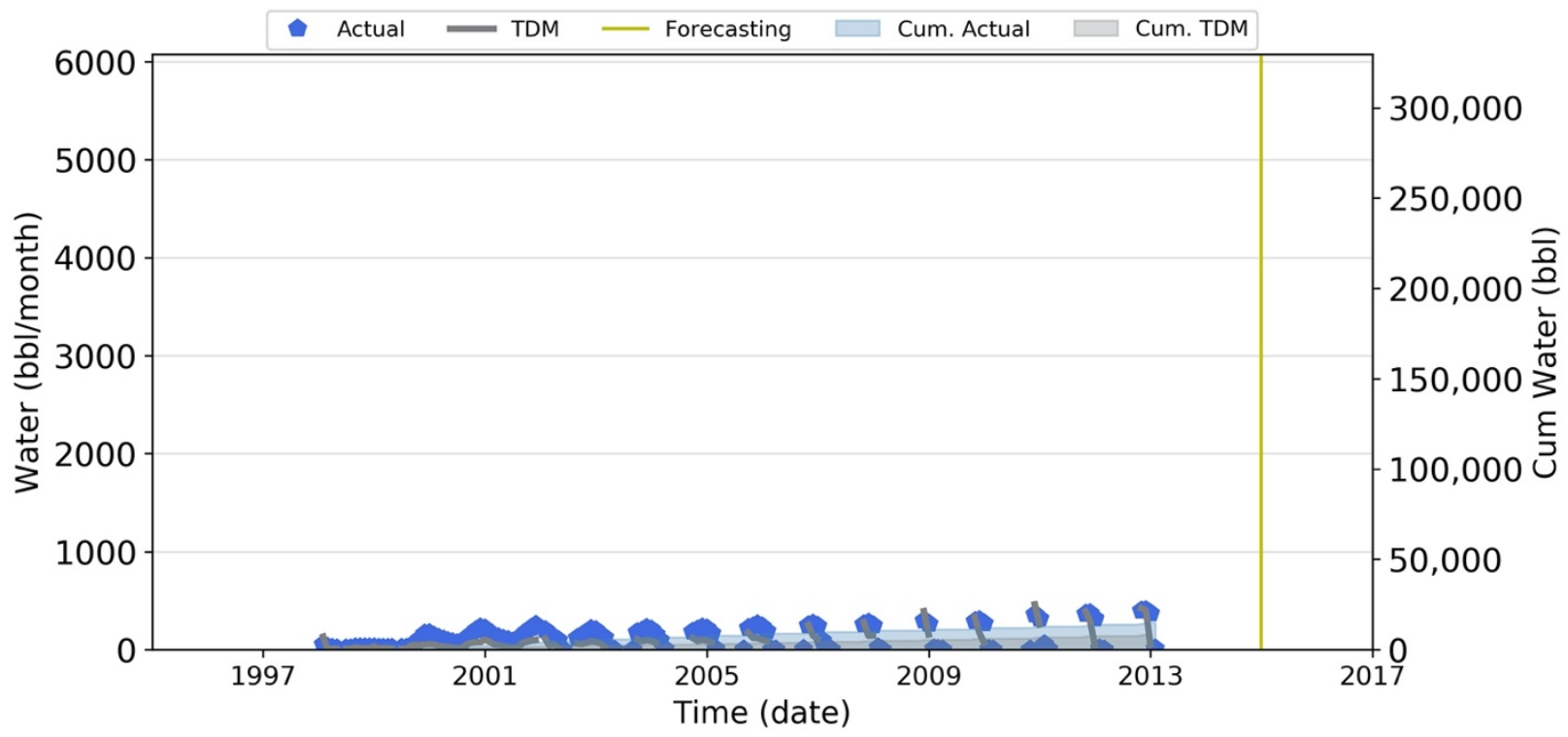


Water: P-21

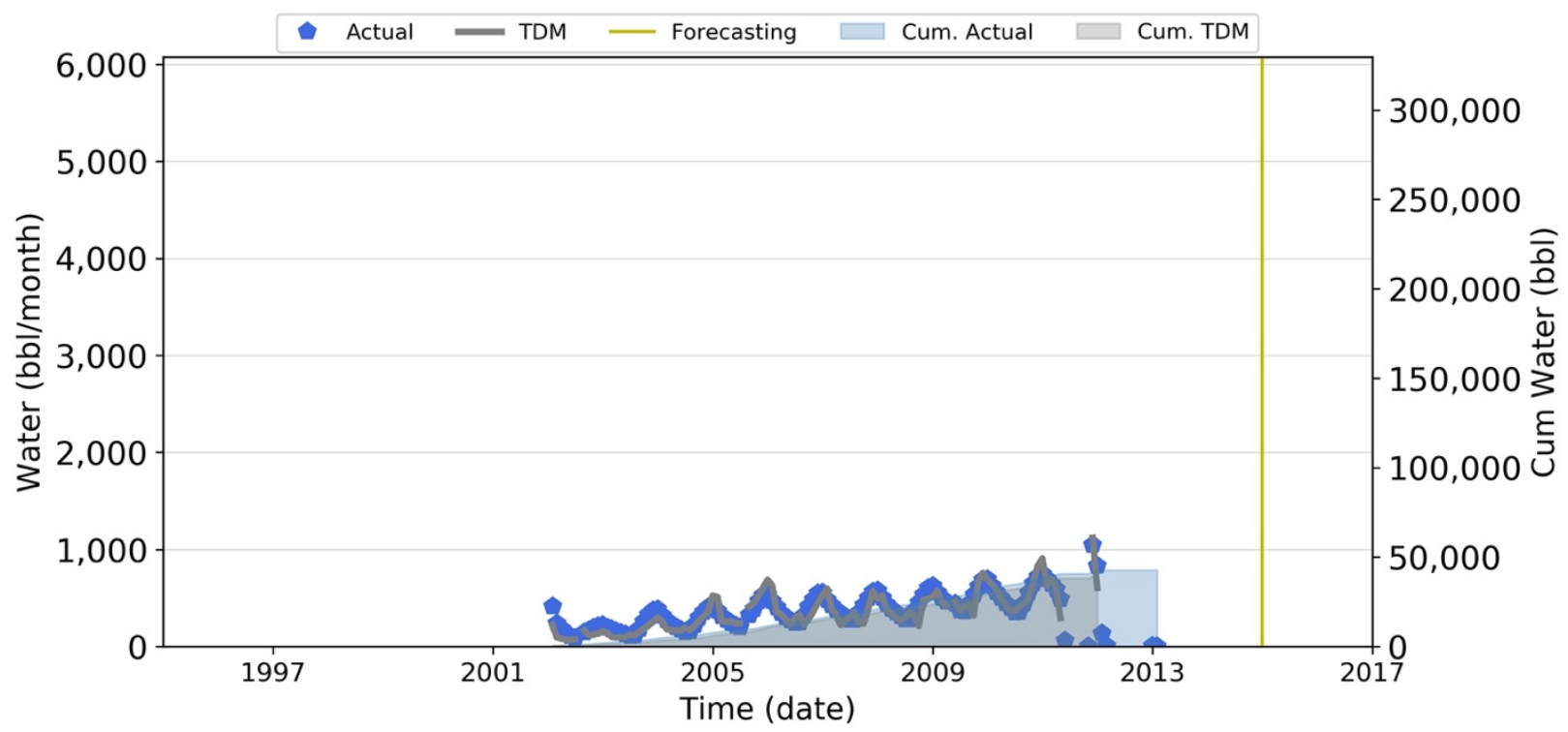

Water: P-22

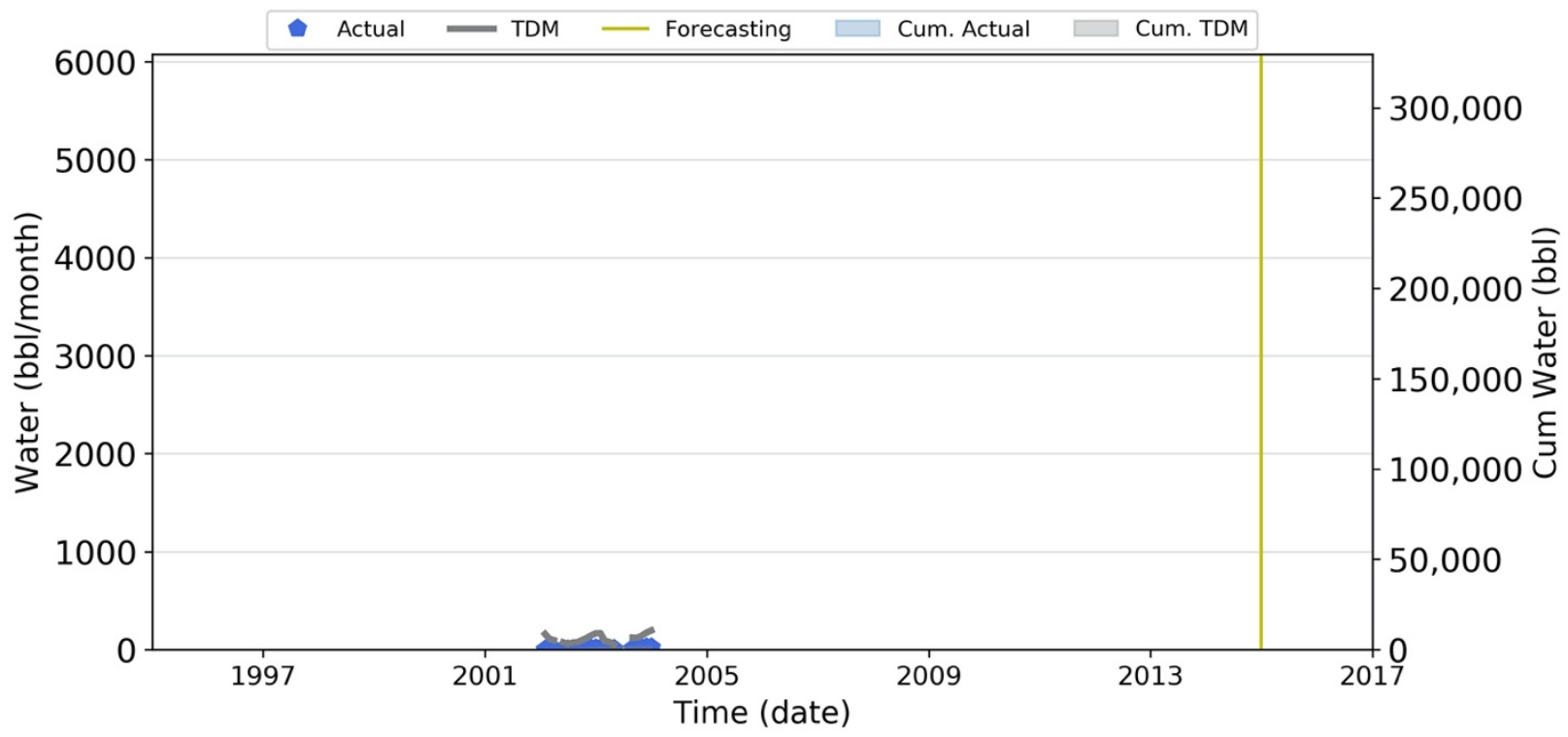


Water: P-23

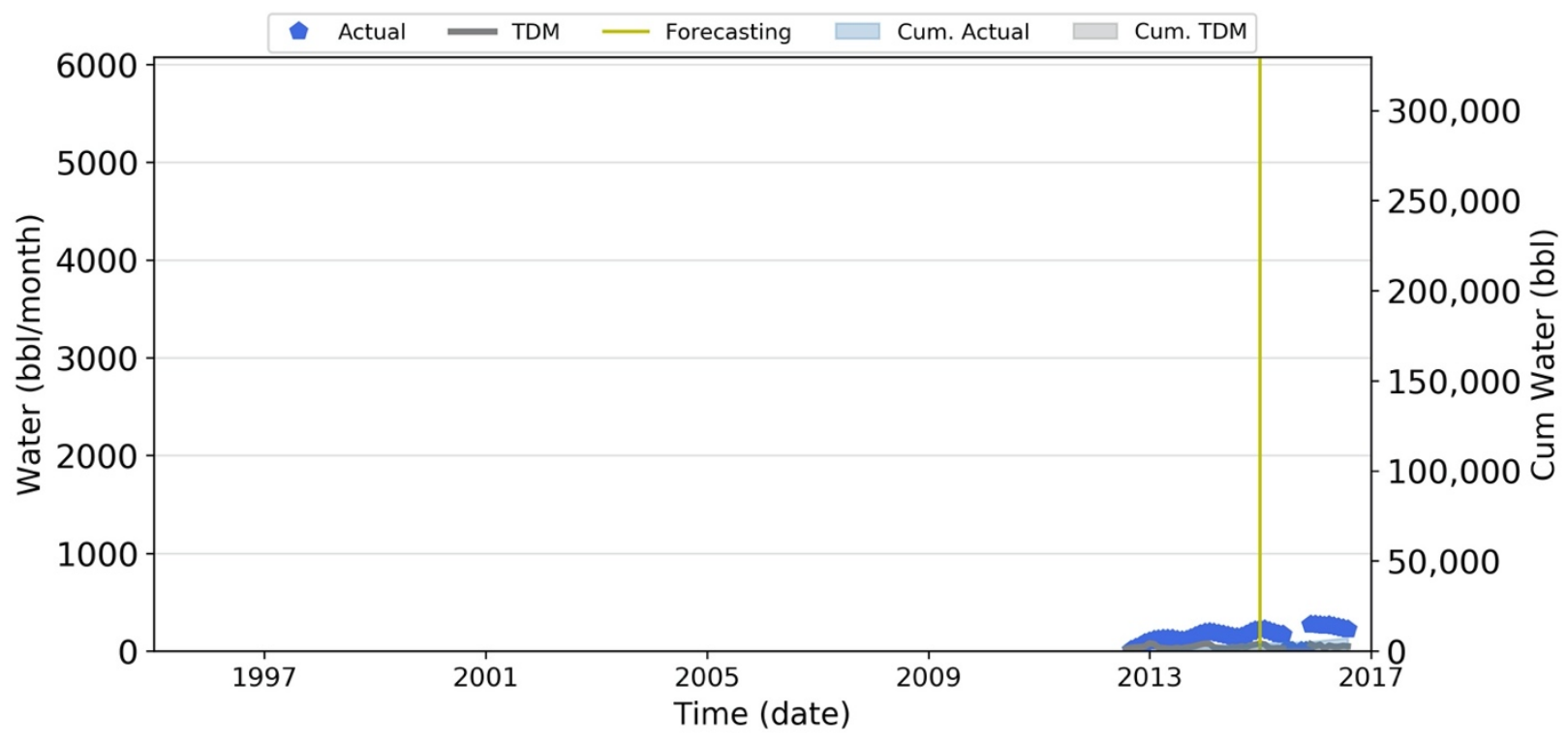

Water: P-24

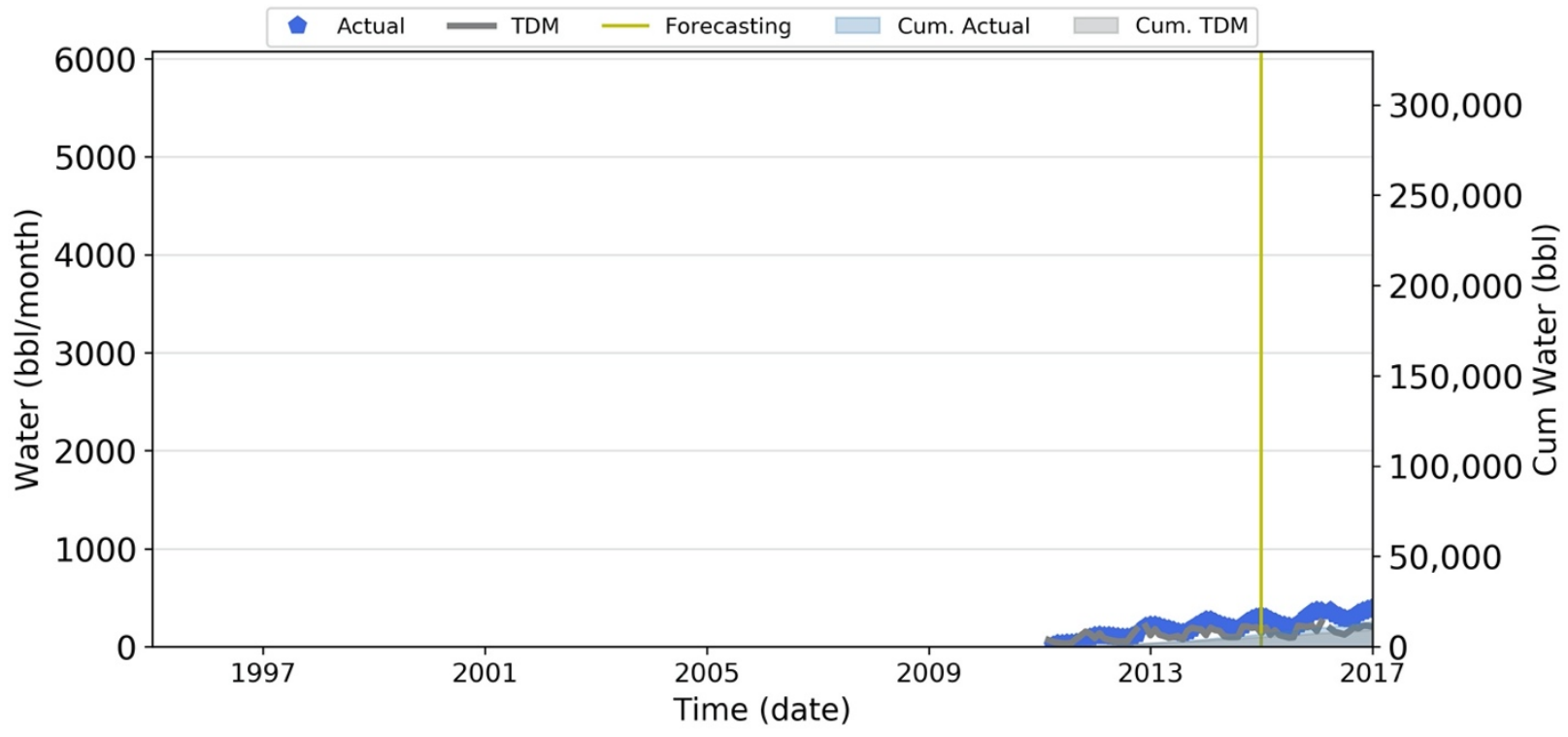


Water: P-25
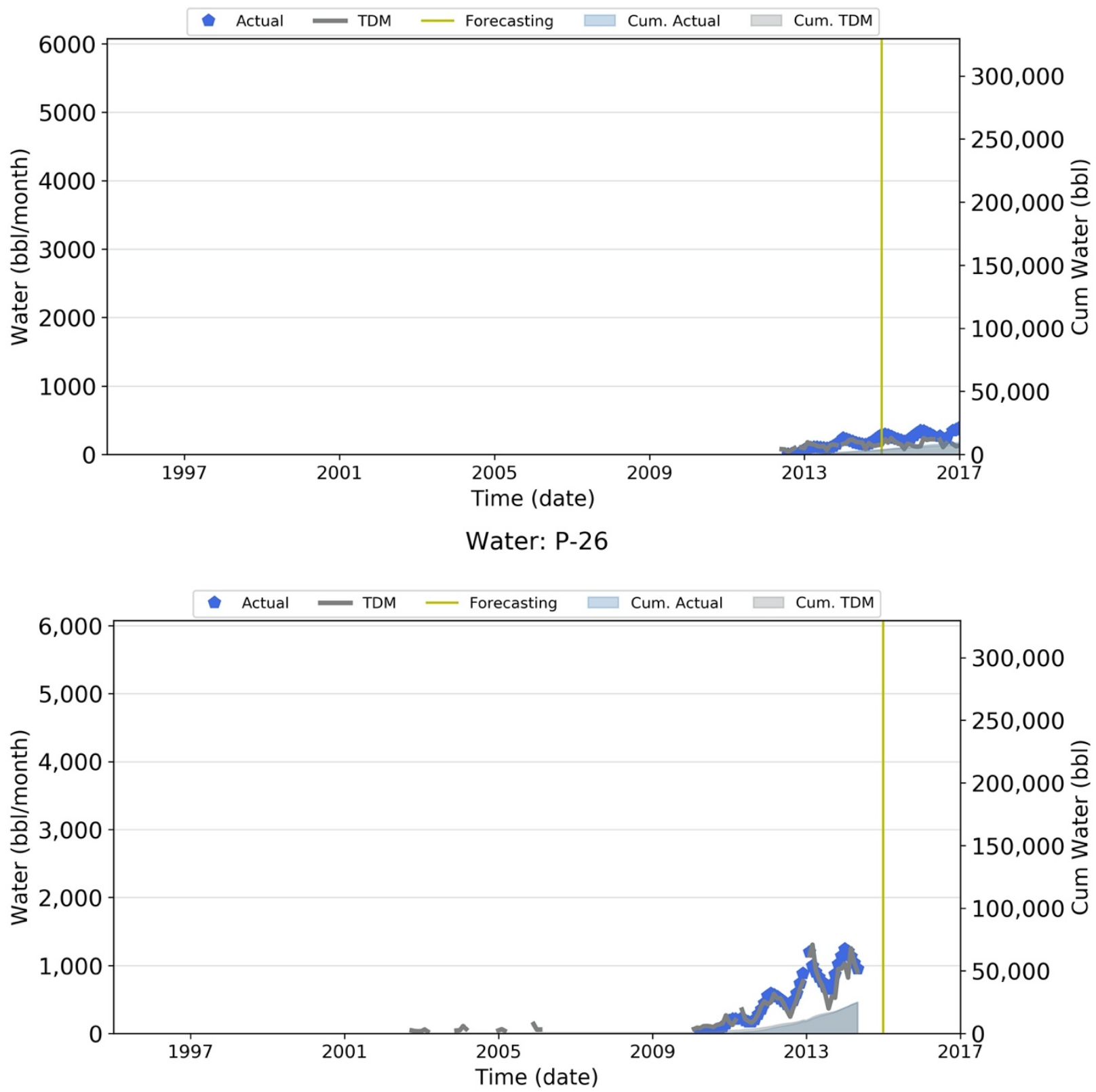
Water: P-27

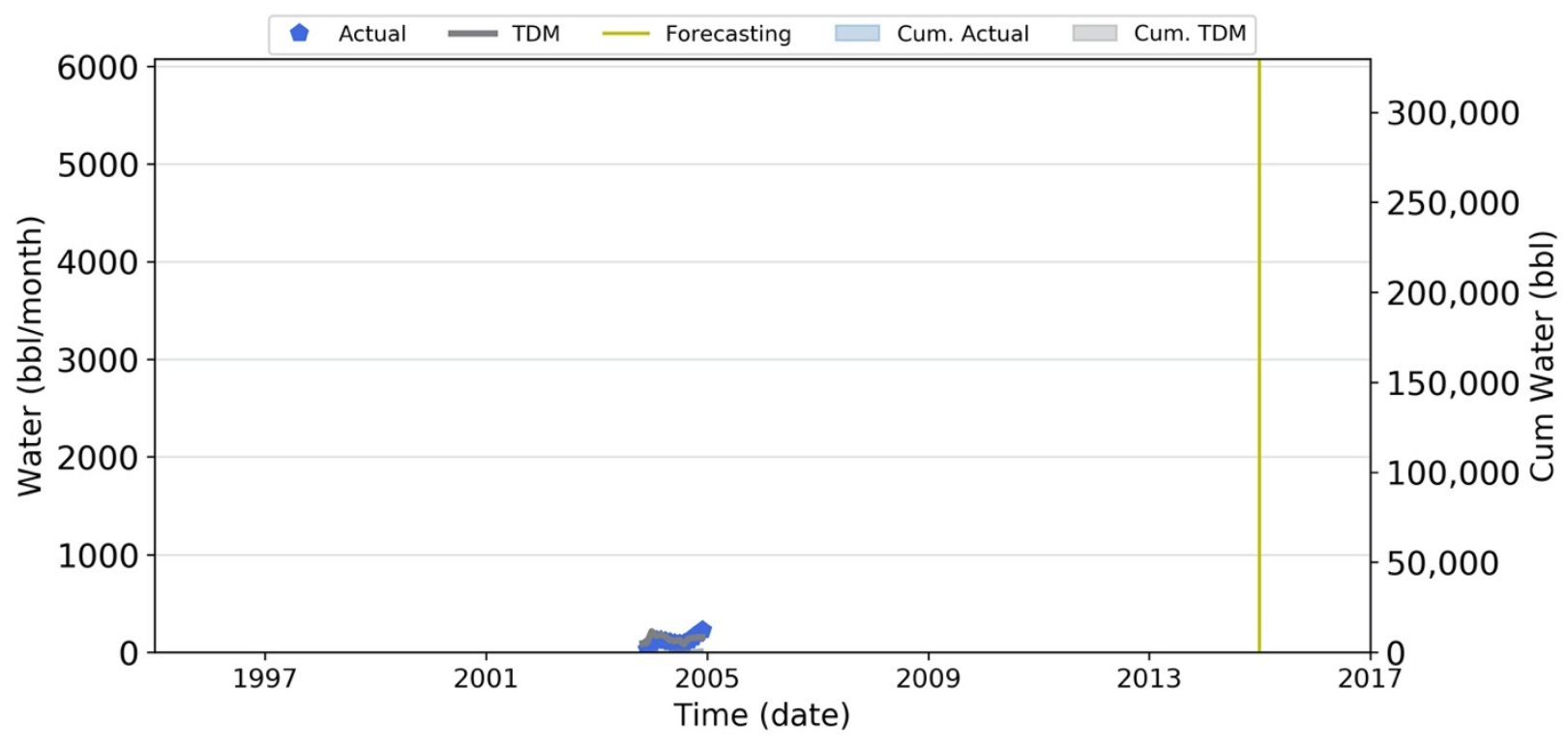

Water: P-28

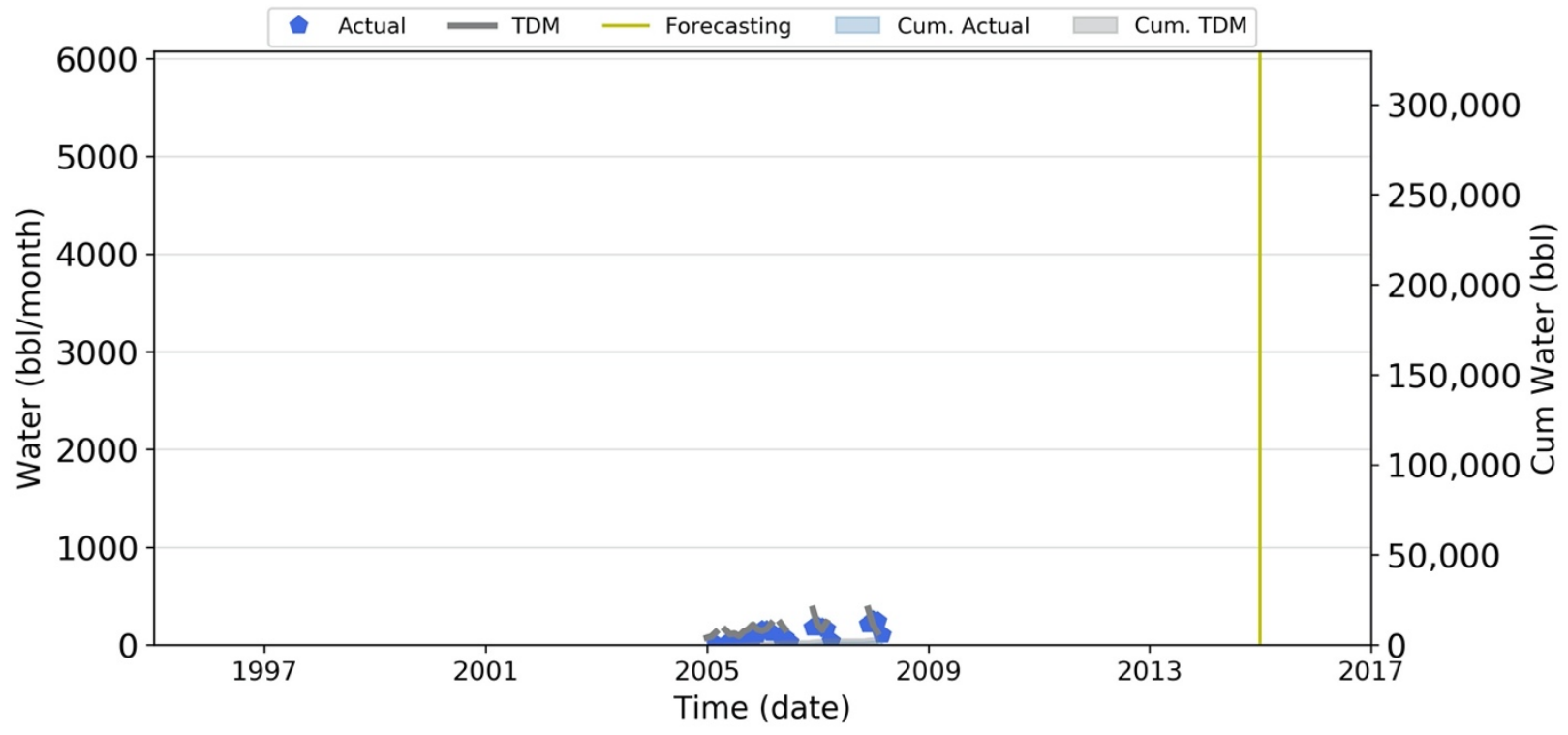


Water: P-29

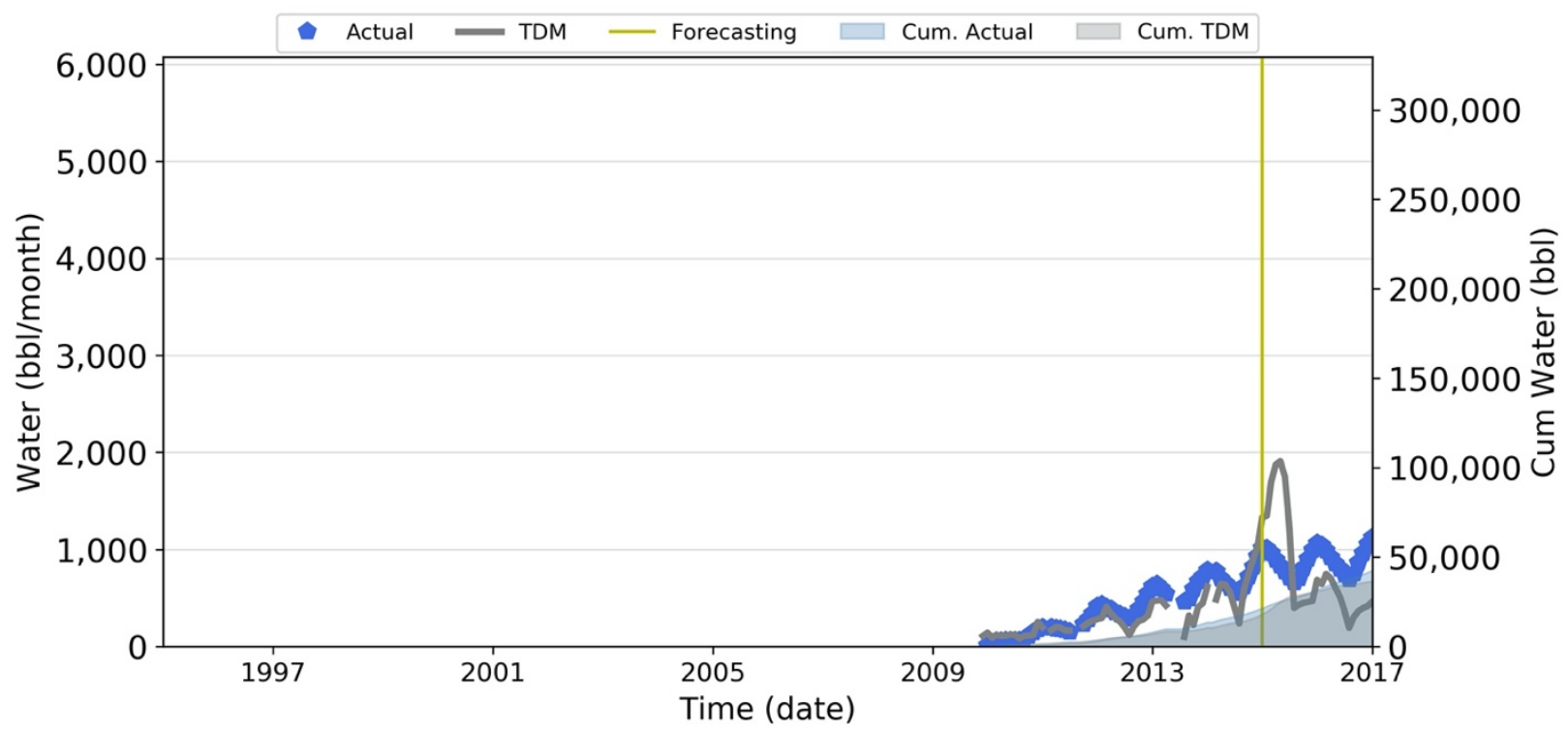

Water: P-30

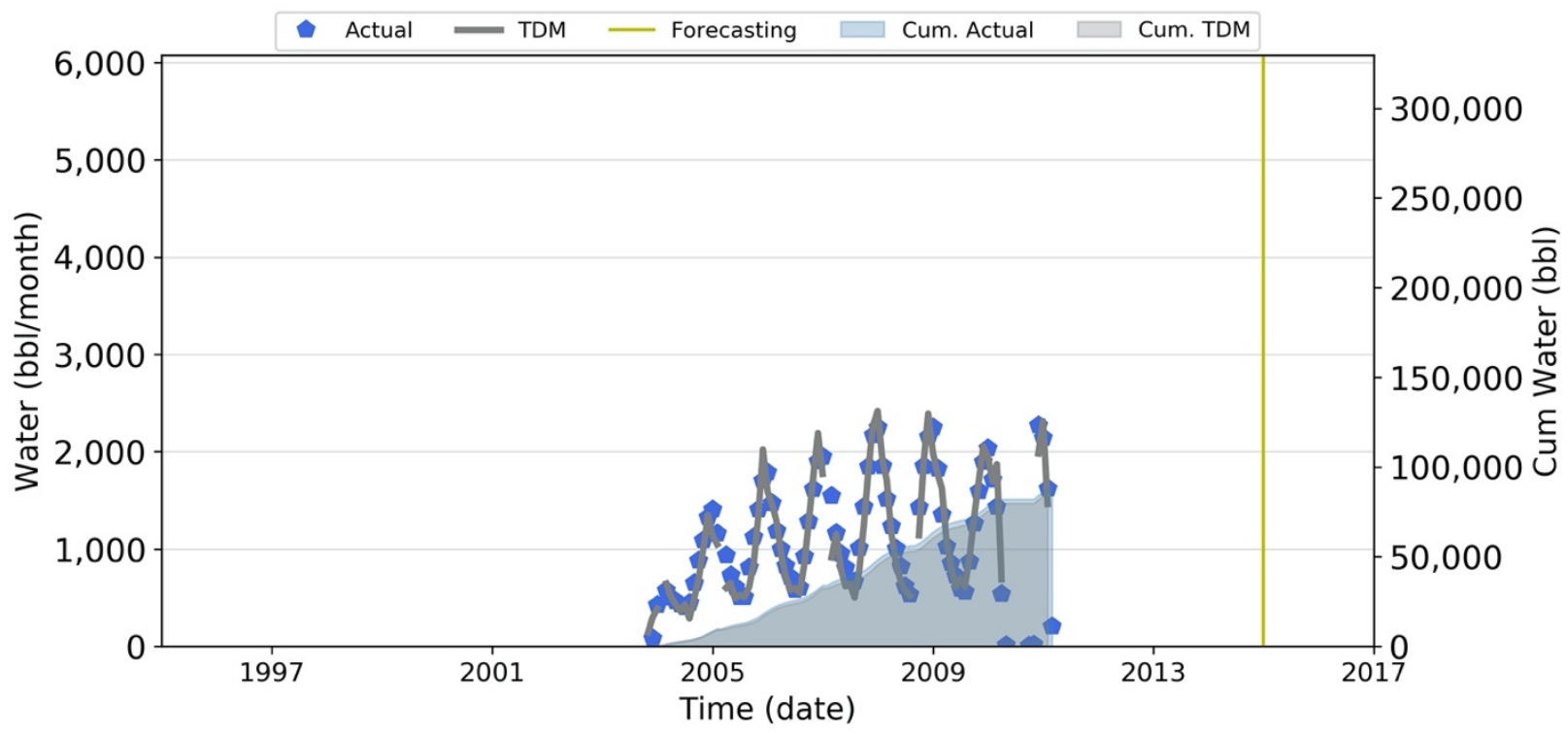


Water: P-31

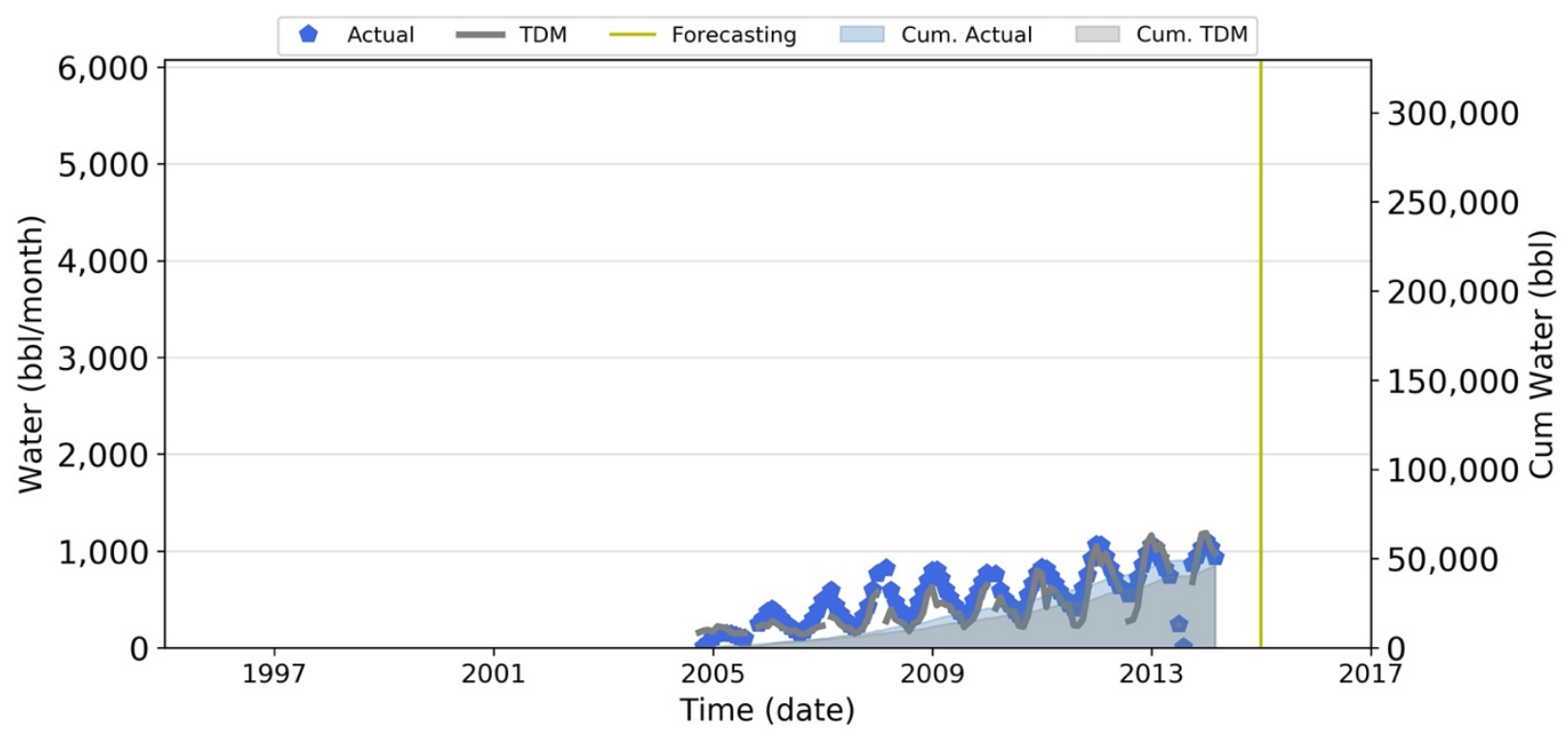

Water: P-32

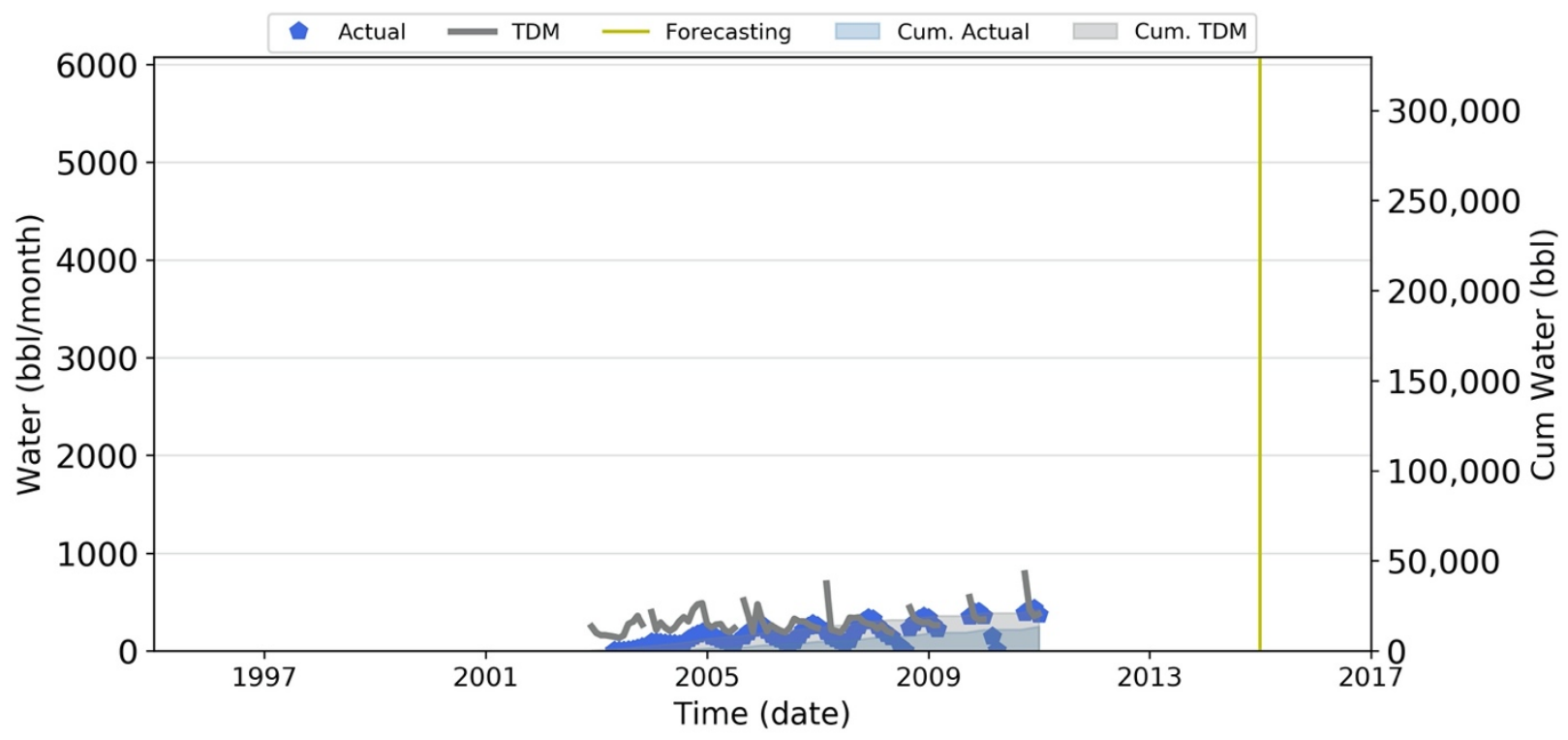


Water: P-33

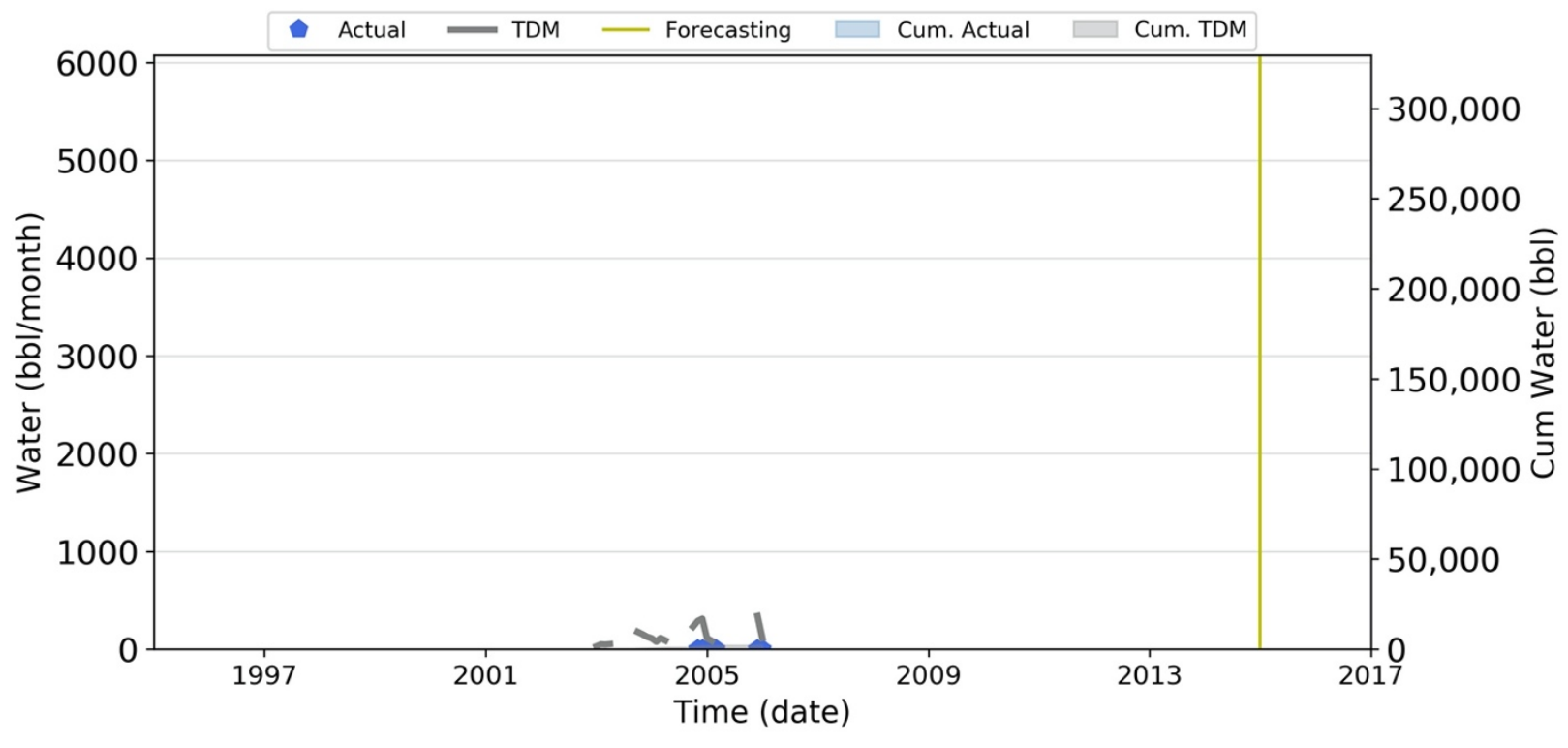

Water: P-34

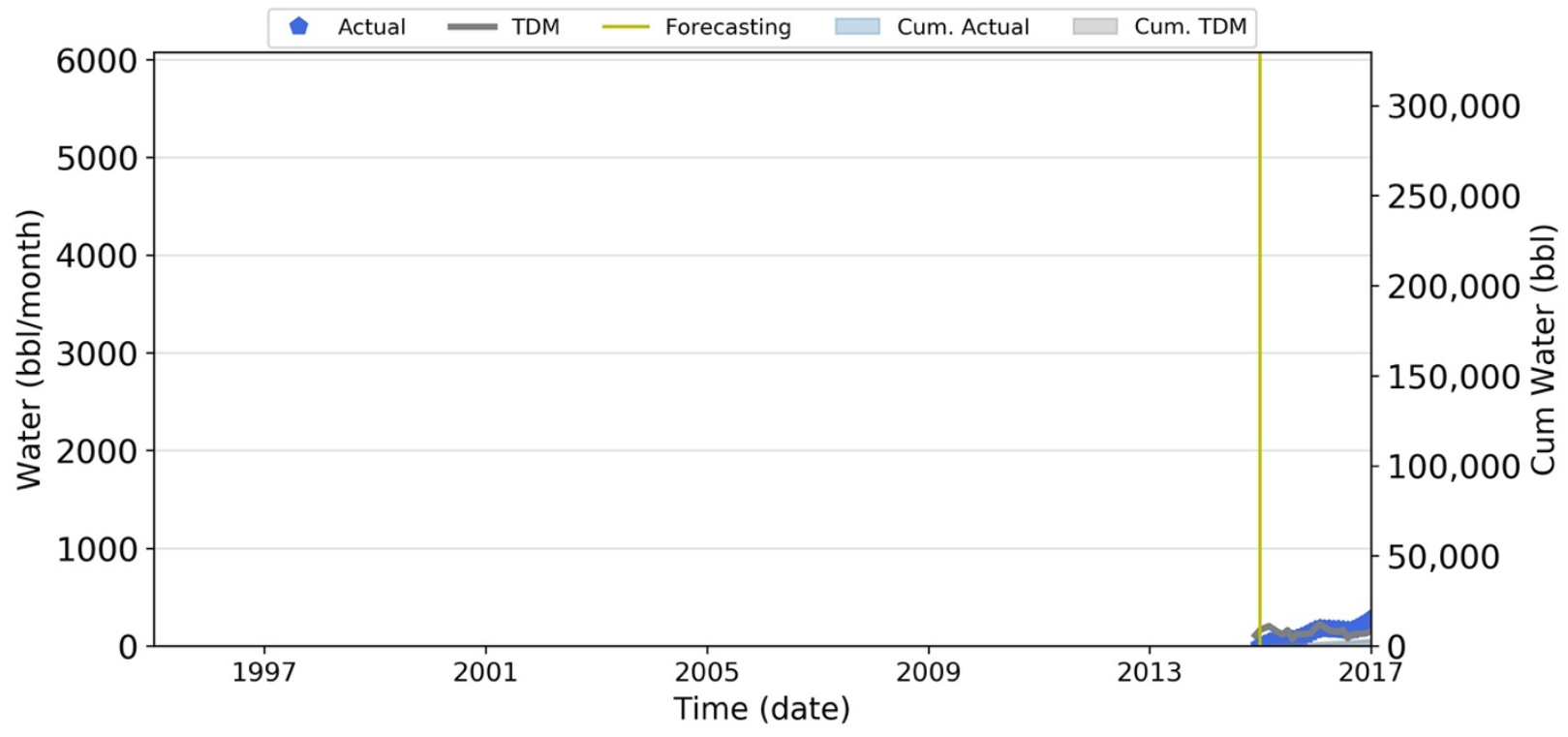


Water: P-37

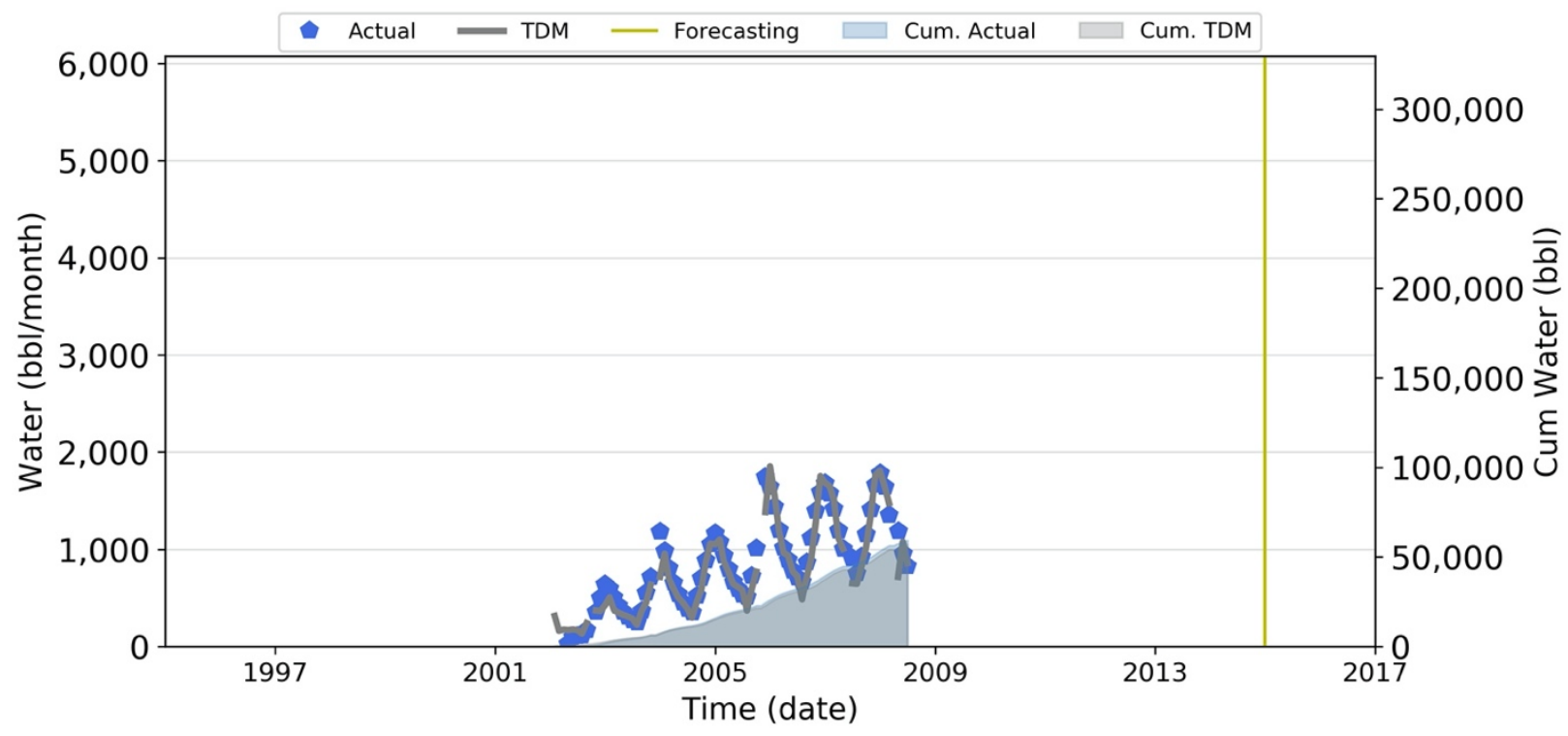

Water: P-38

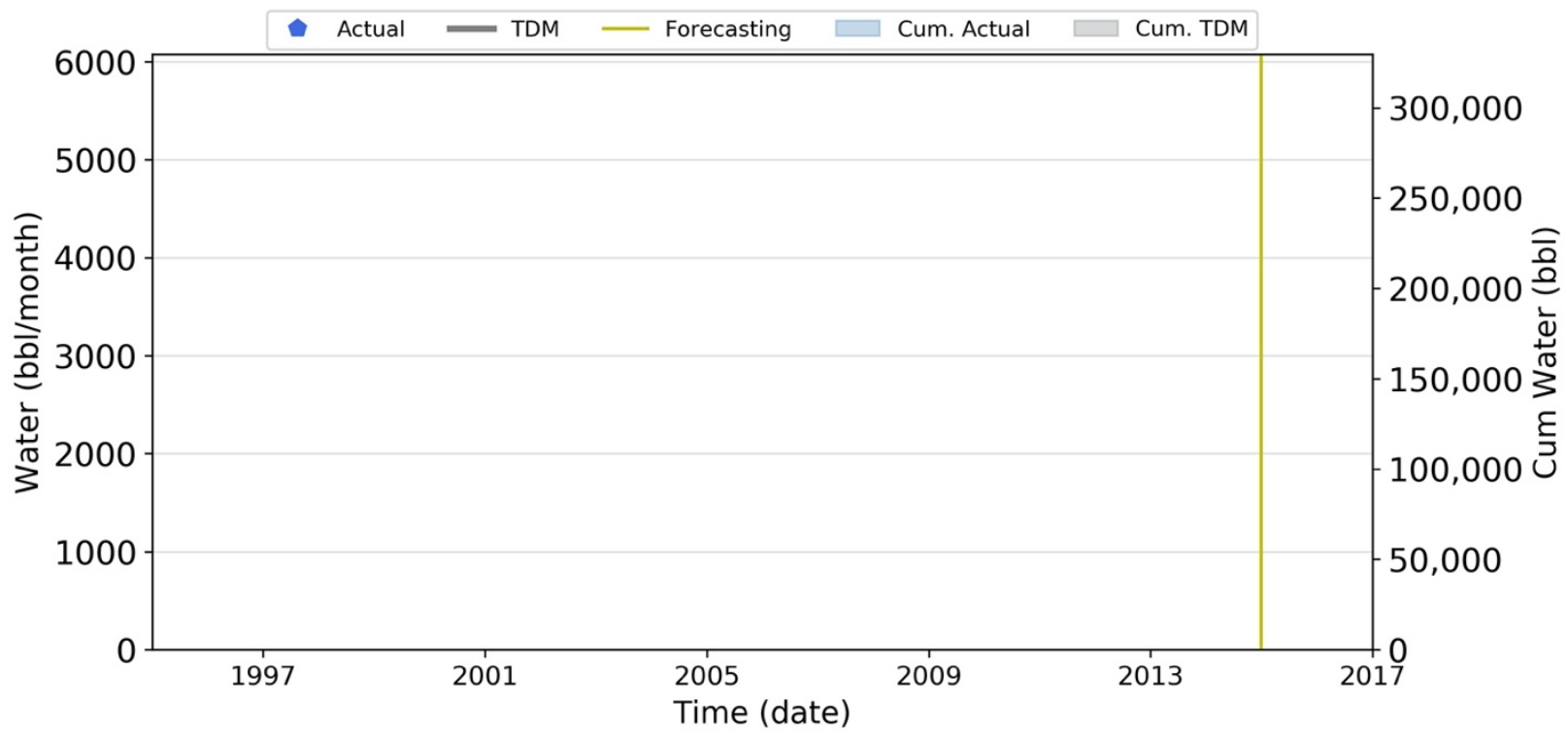


Water: P-39

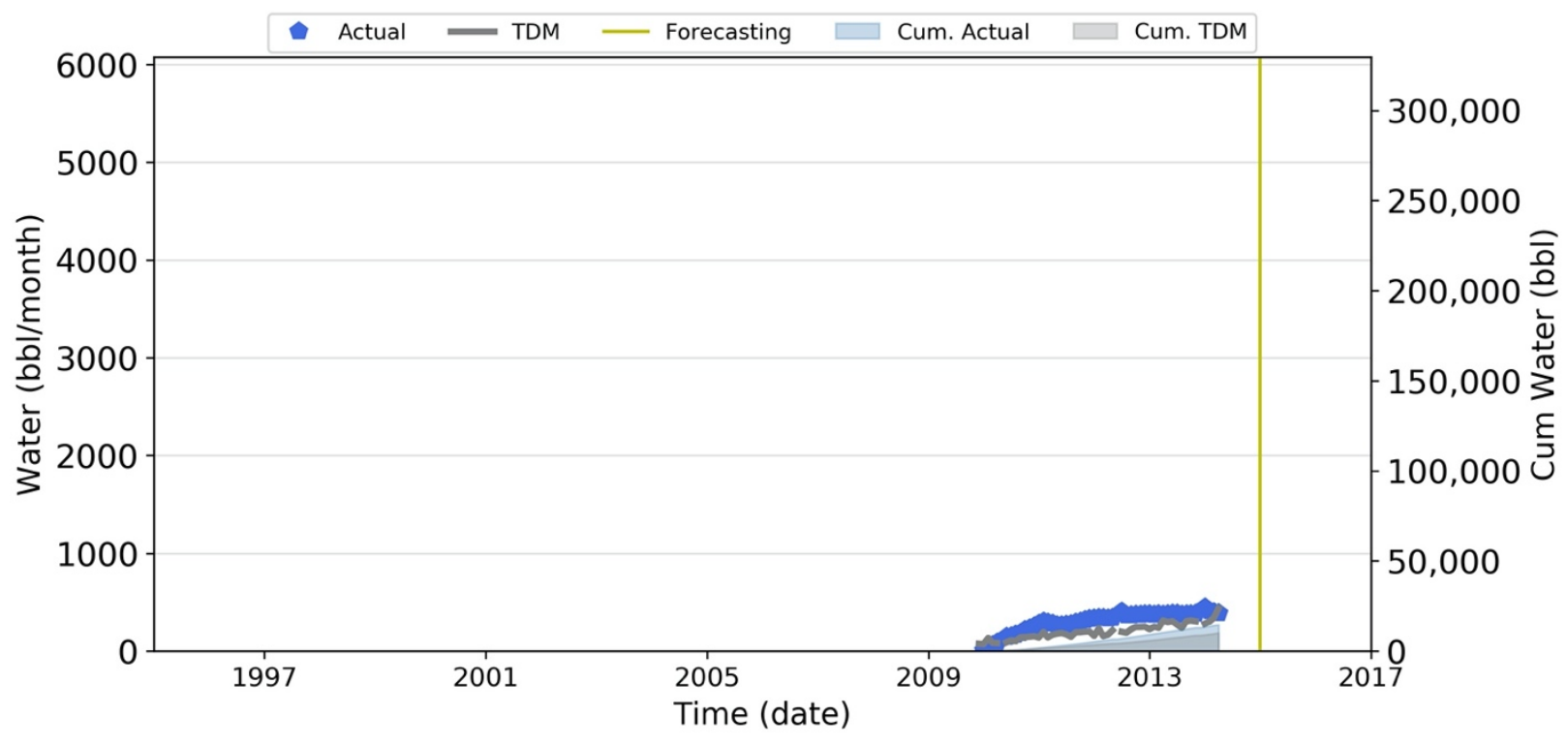

Water: P-41

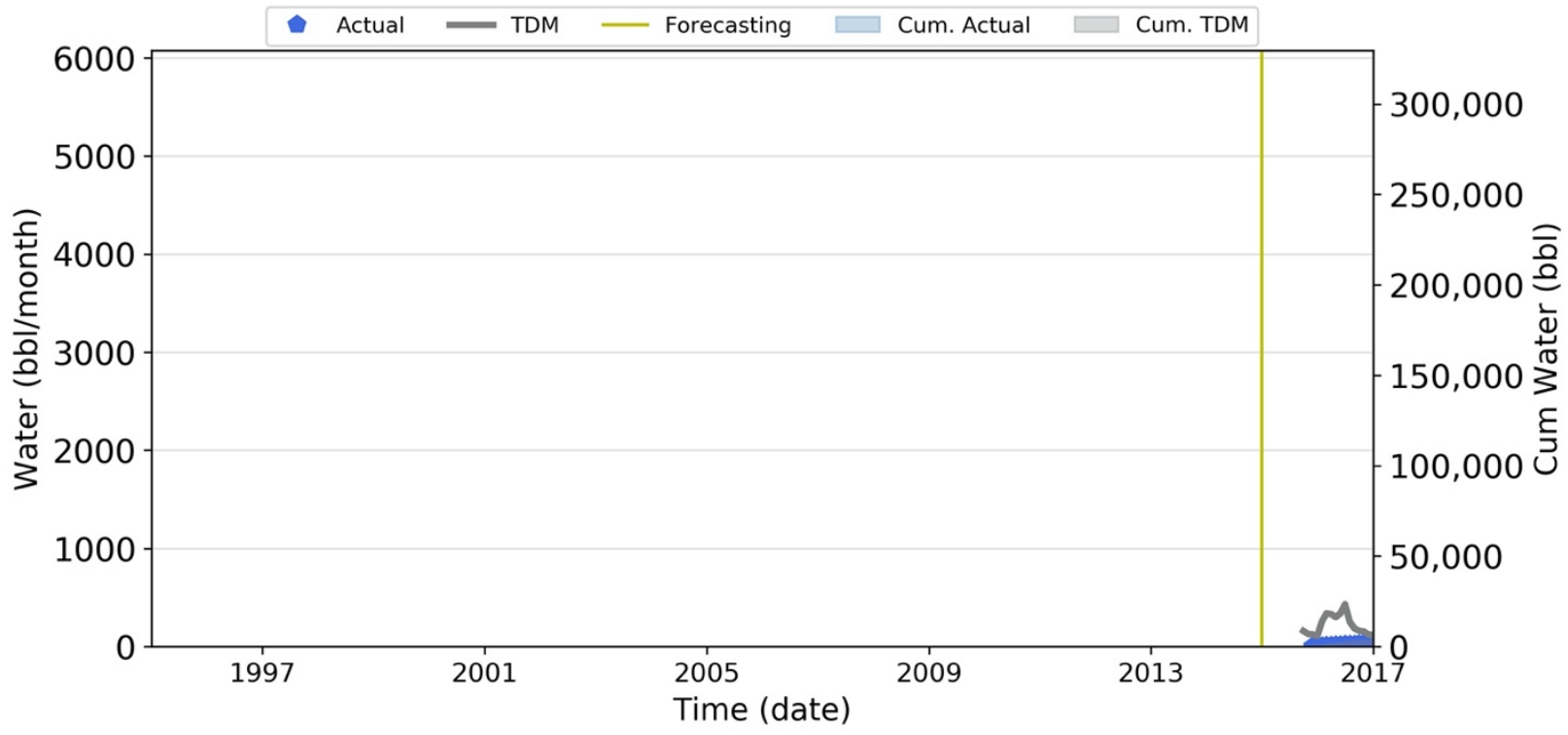


Water: P-42

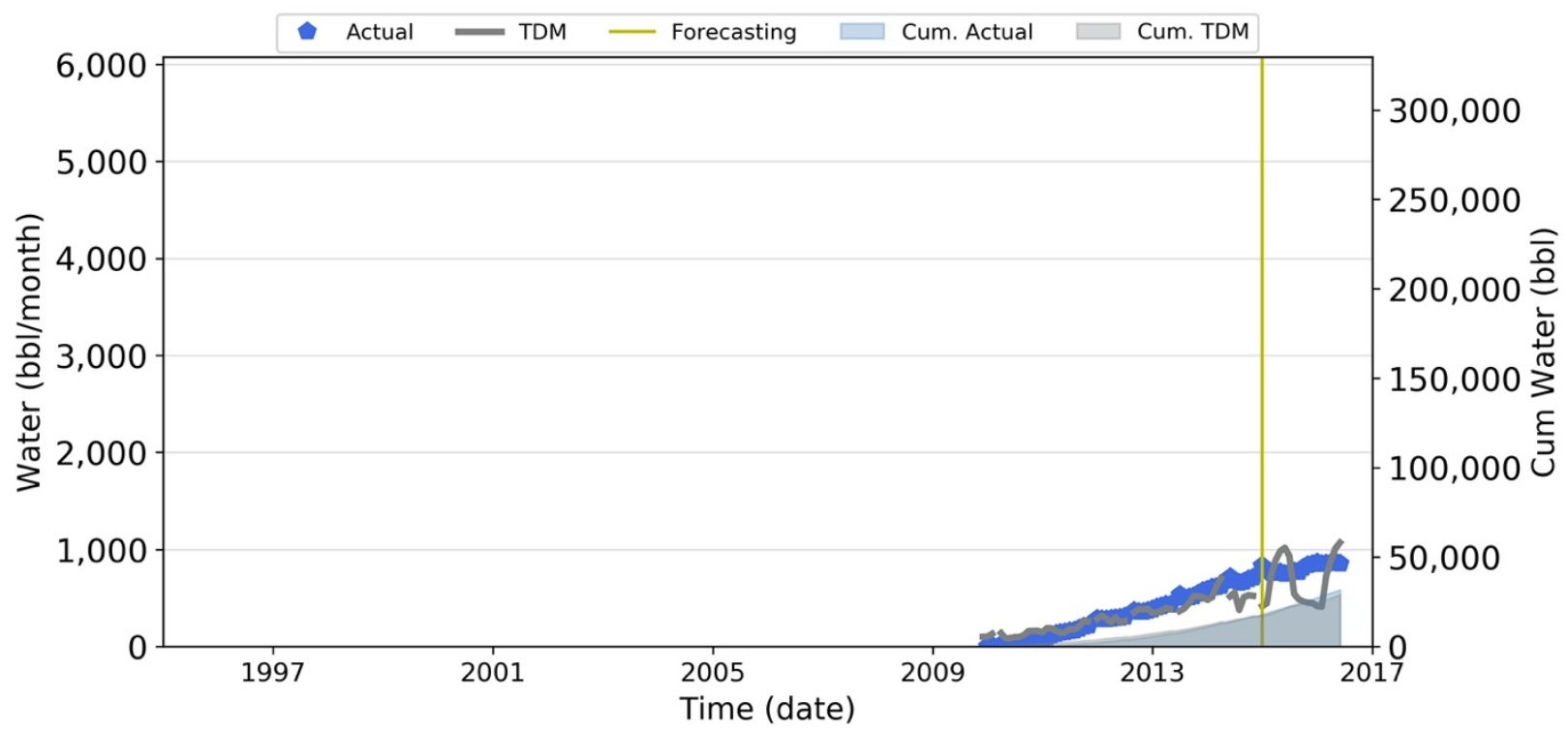

Water: P-43

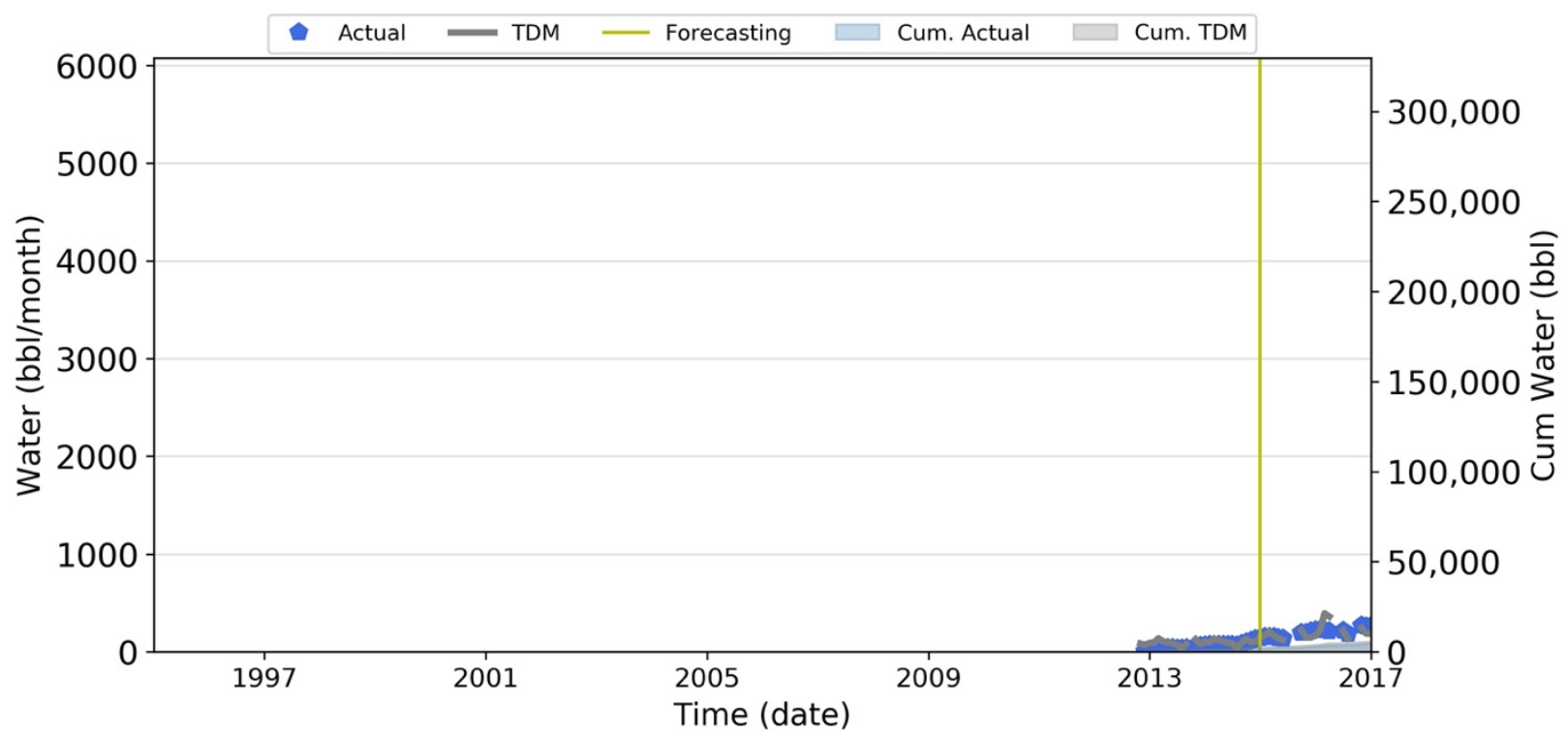


Water: P-44

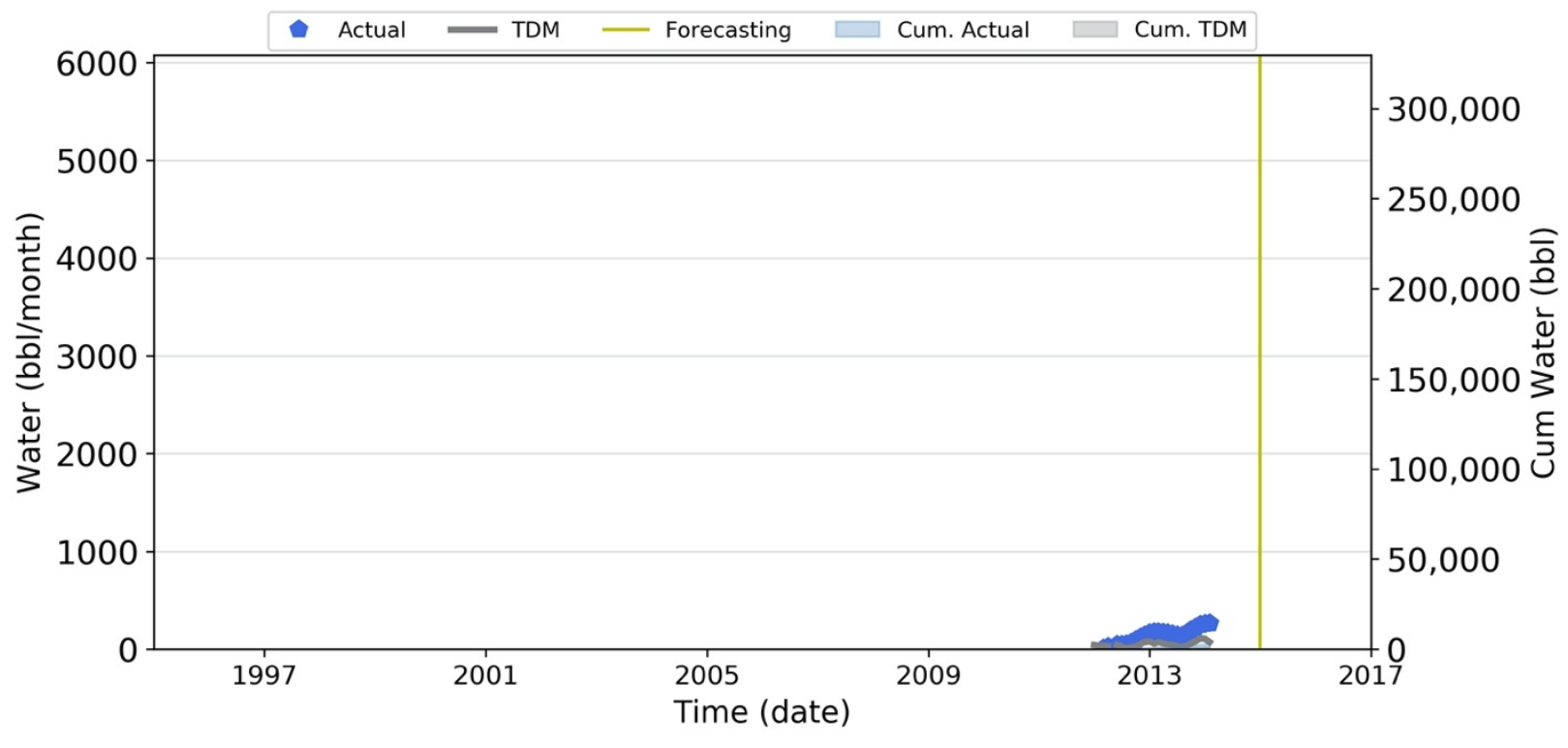

Water: P-47

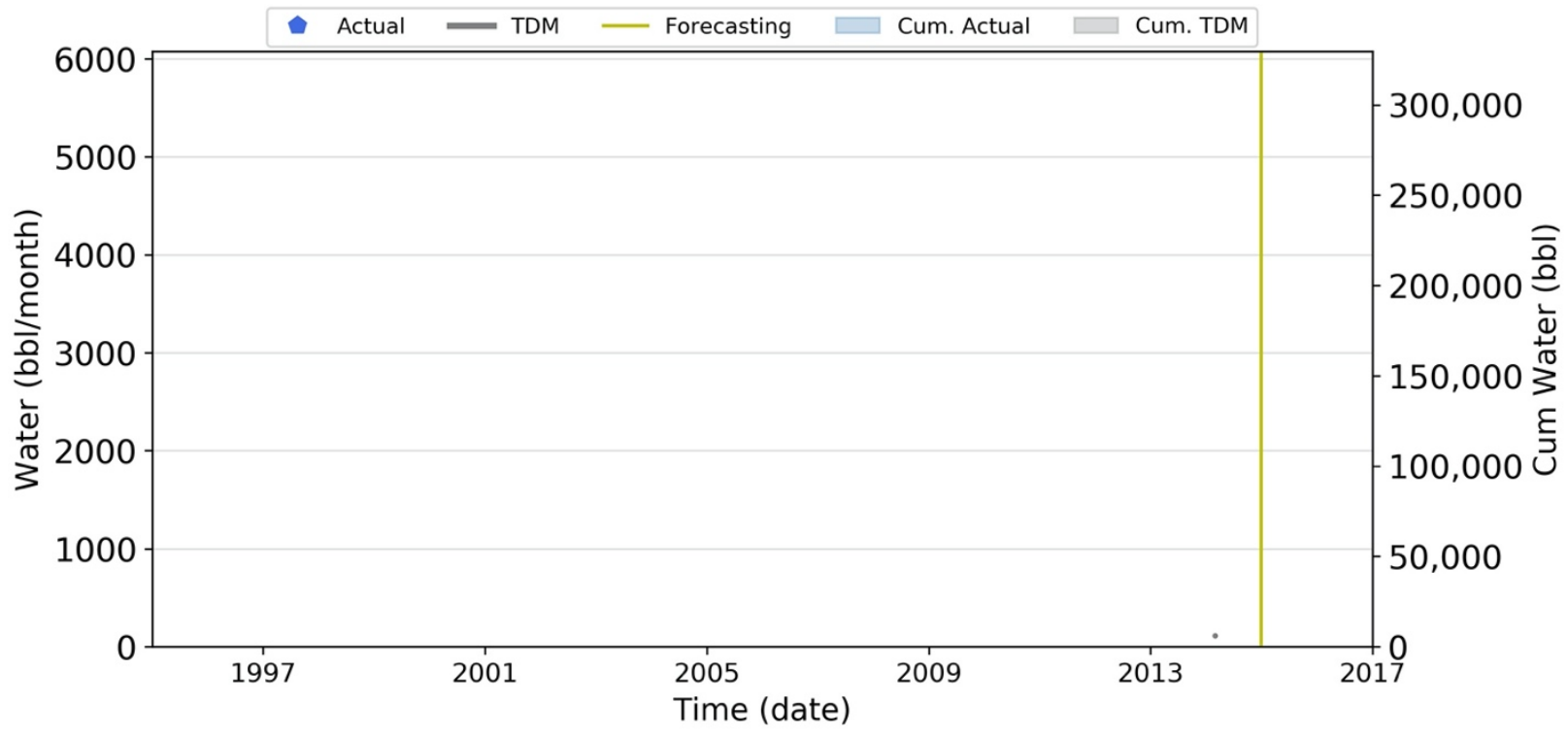


Water: P-49

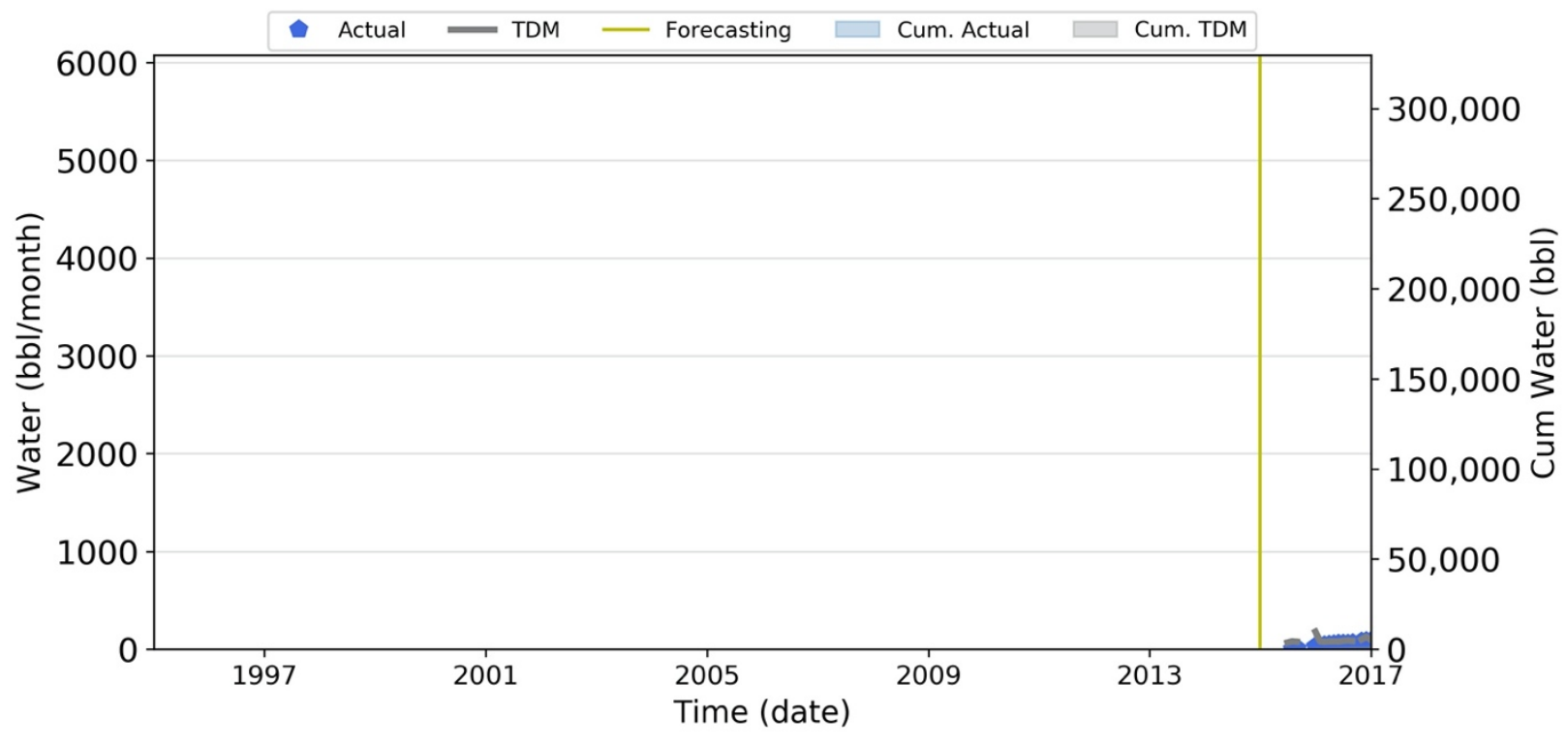

Water: P-50

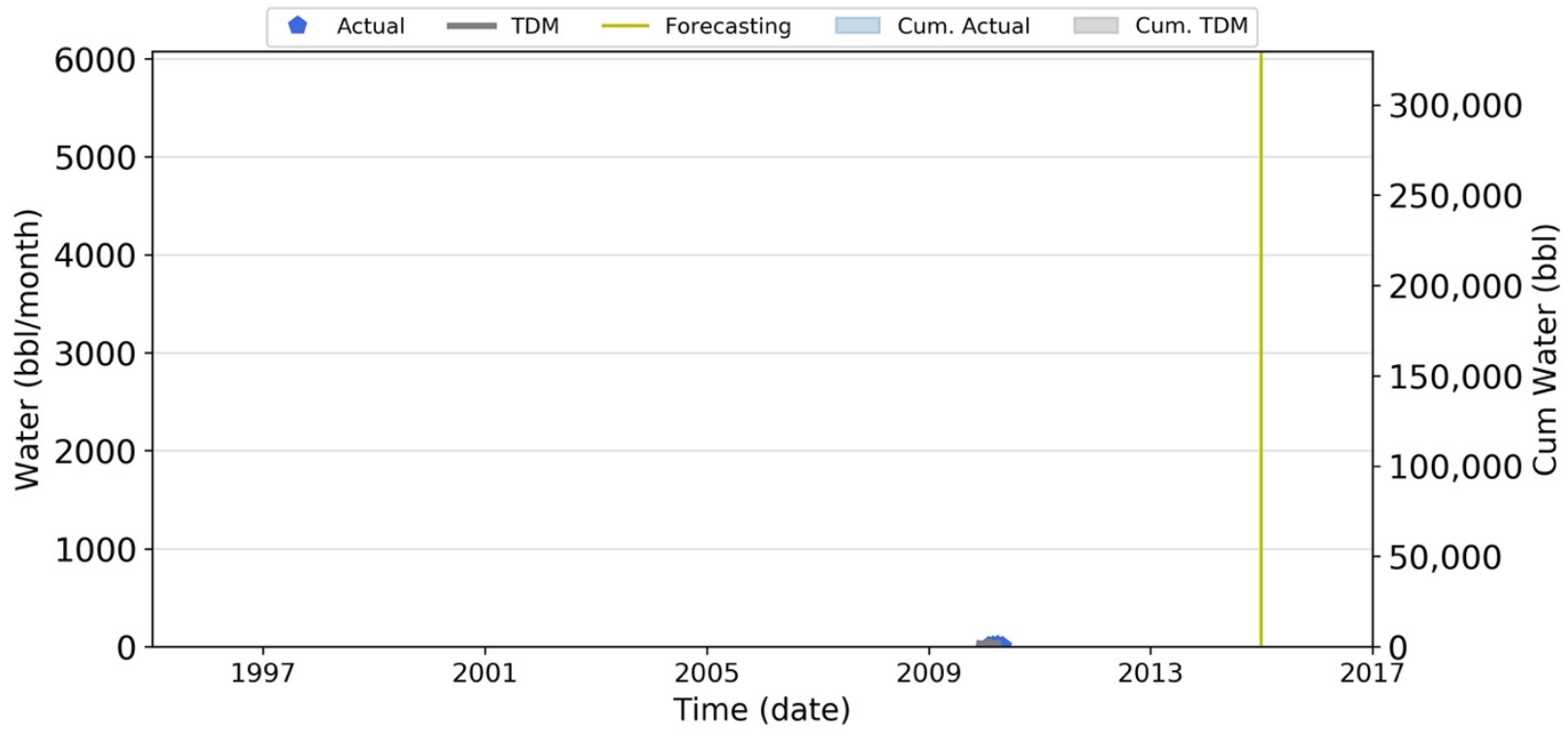


Water: P-51

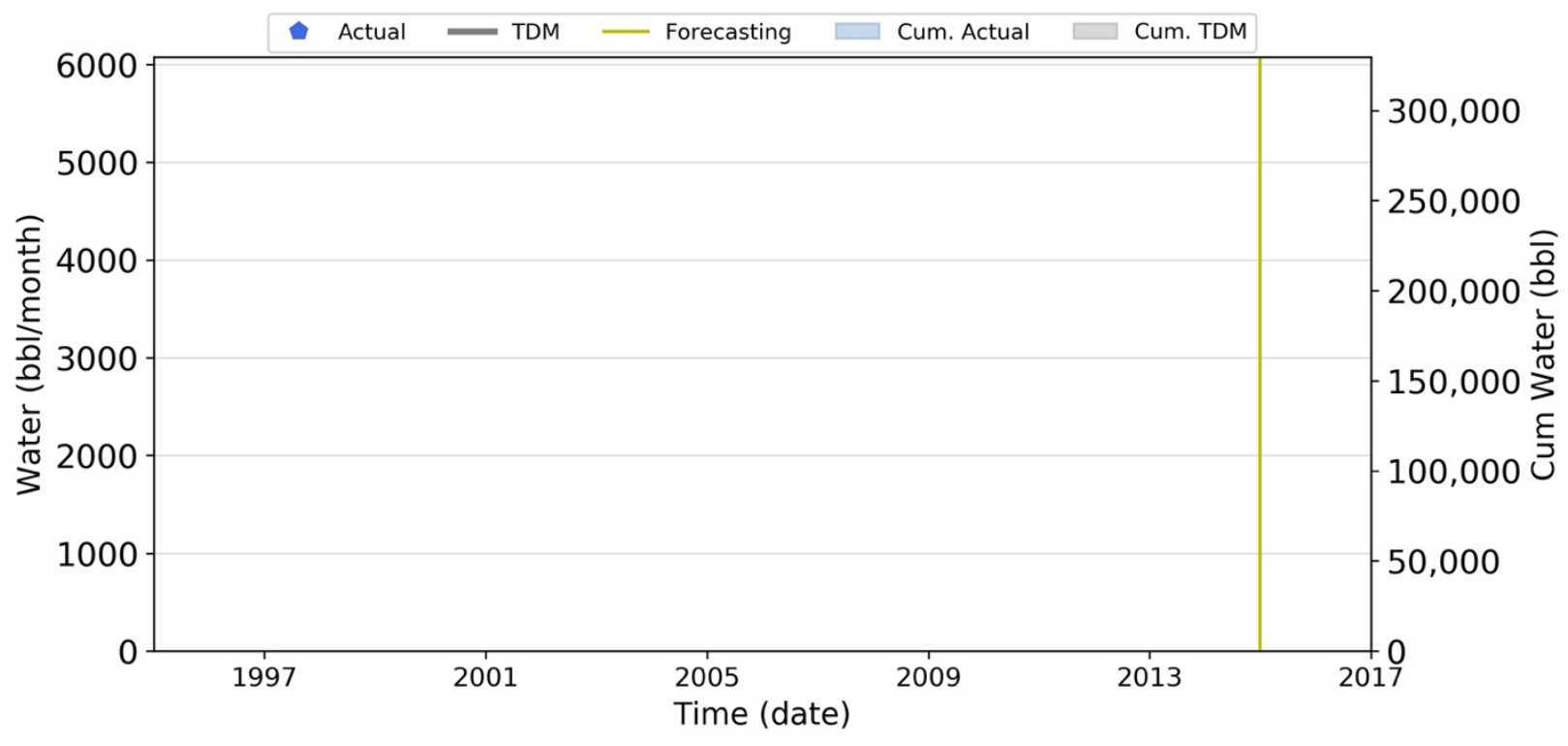

Water: P-52

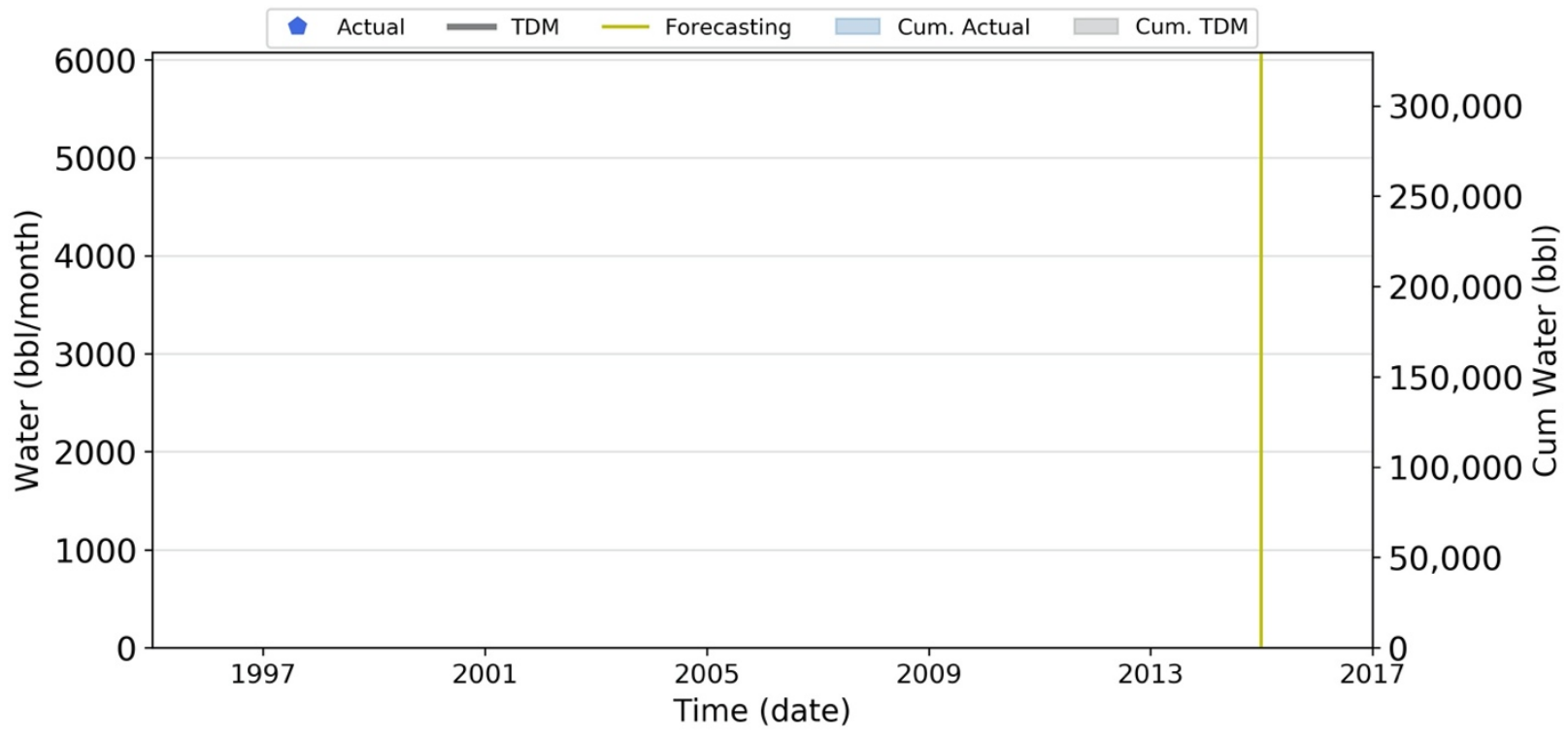


Water: P-53

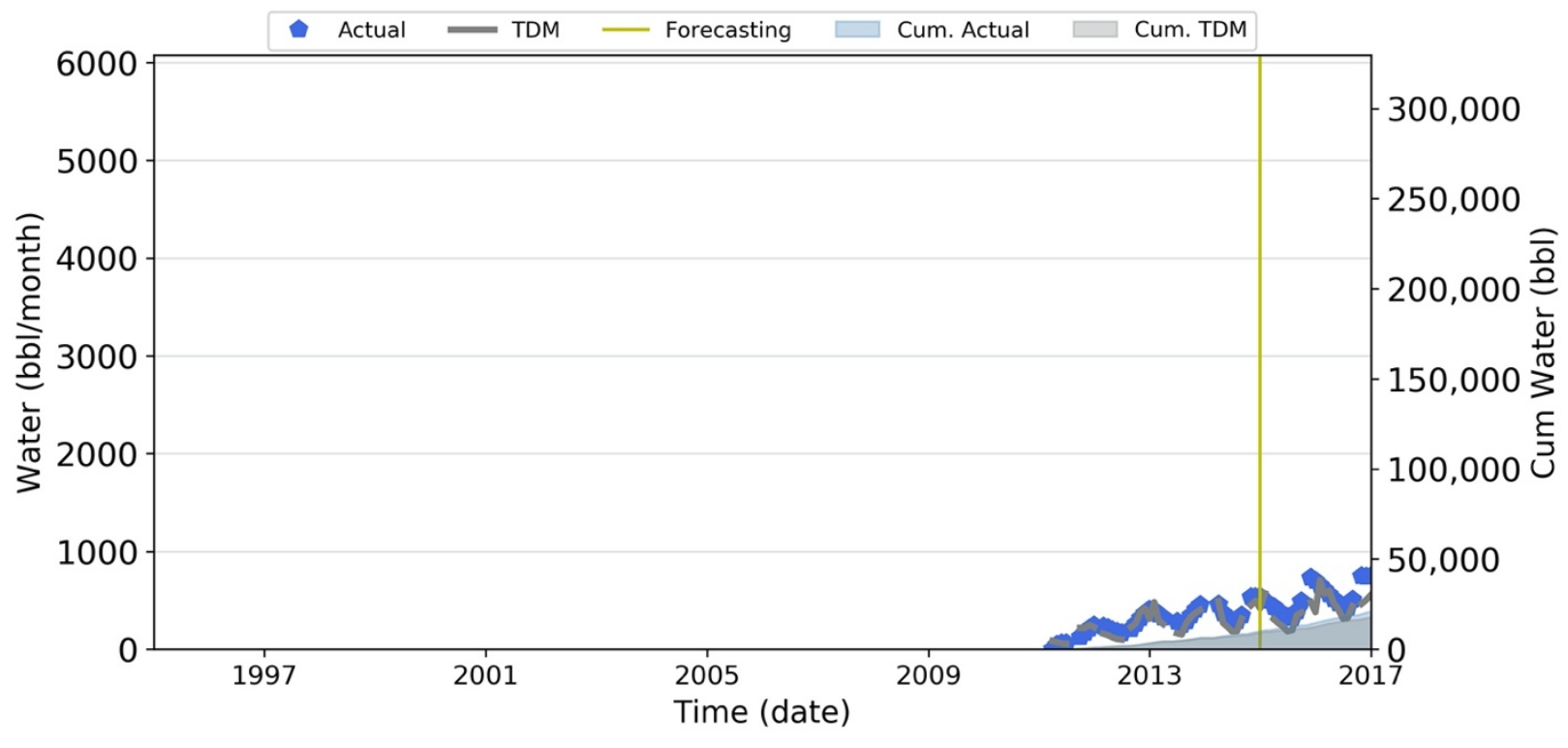

Water: P-54

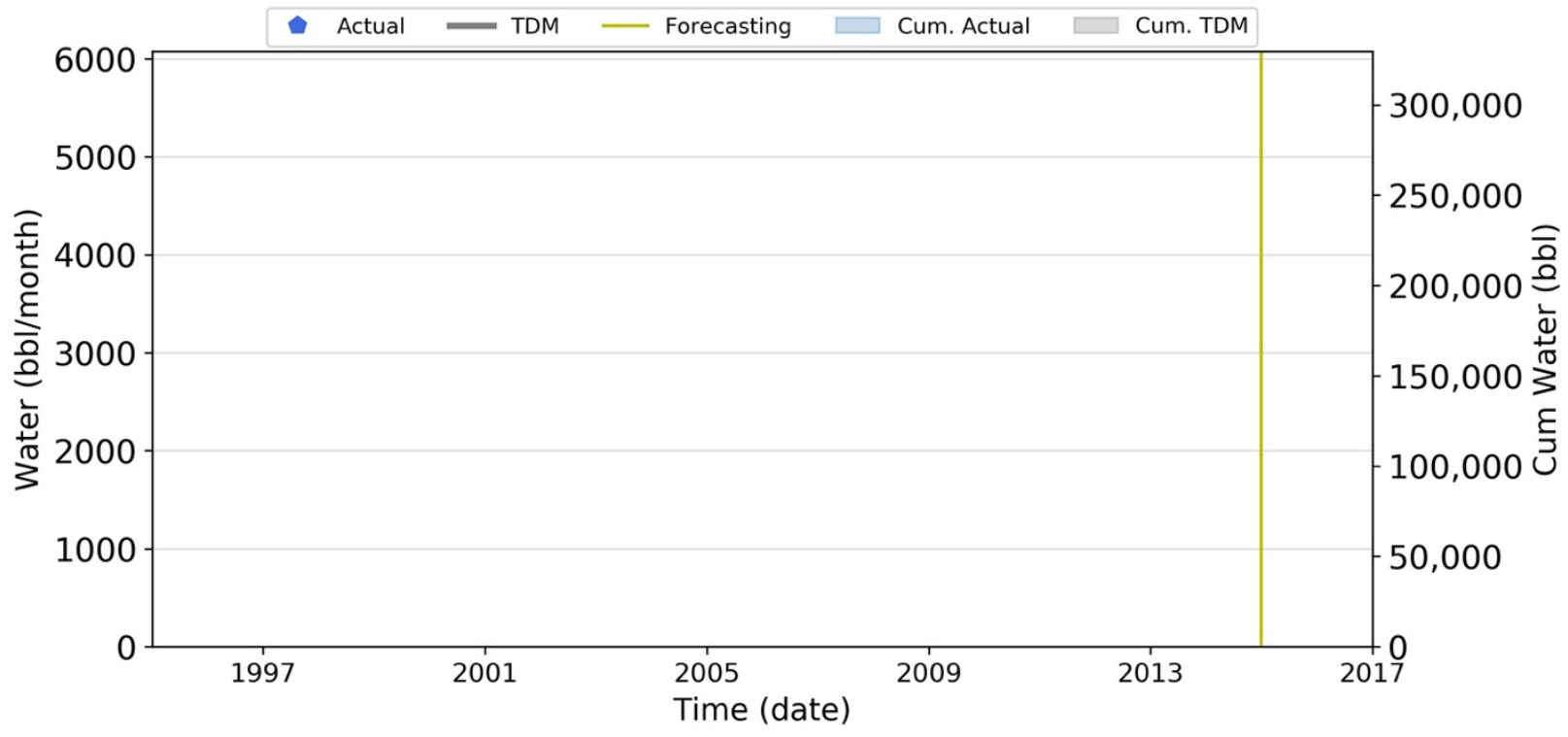


Water: P-55

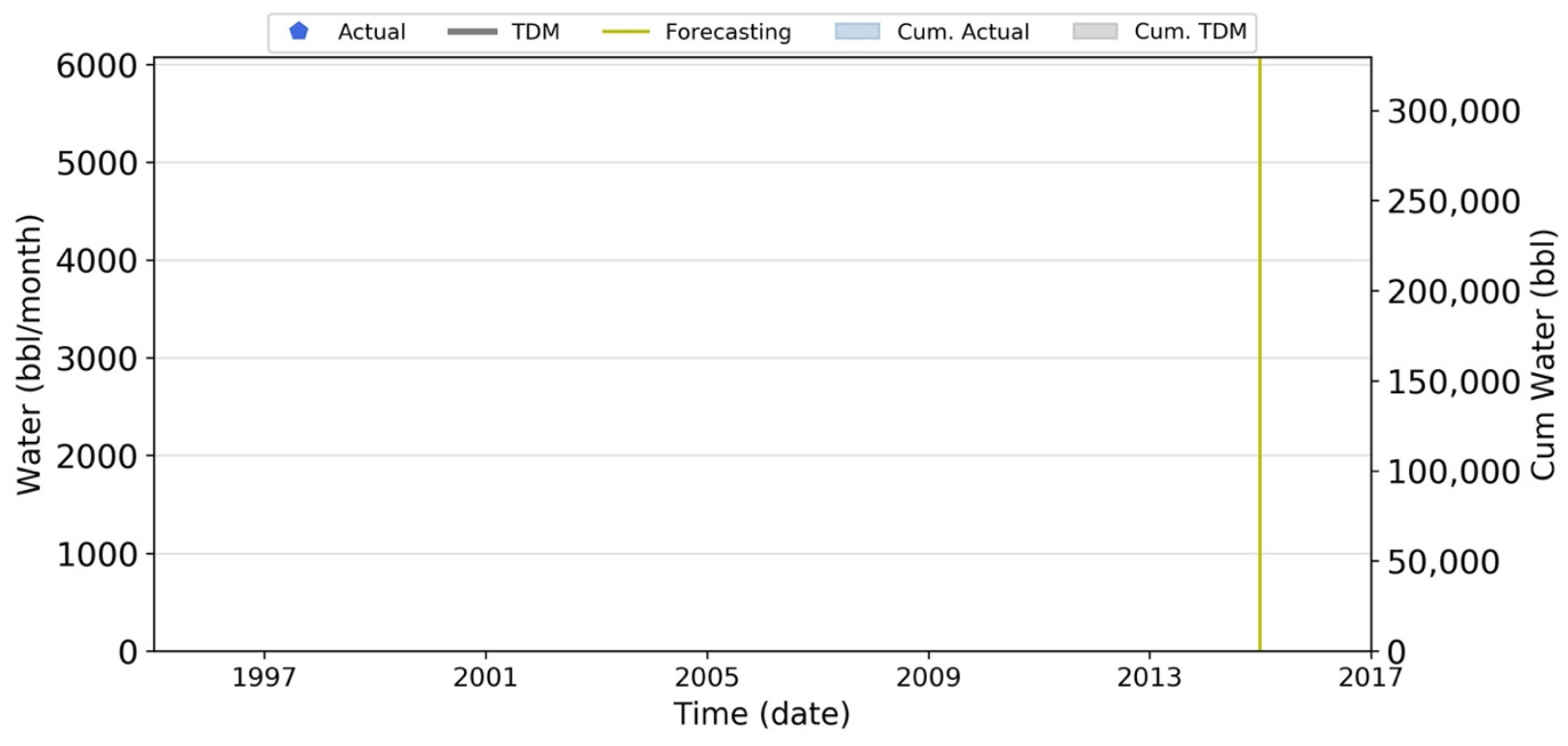

Water: P-56

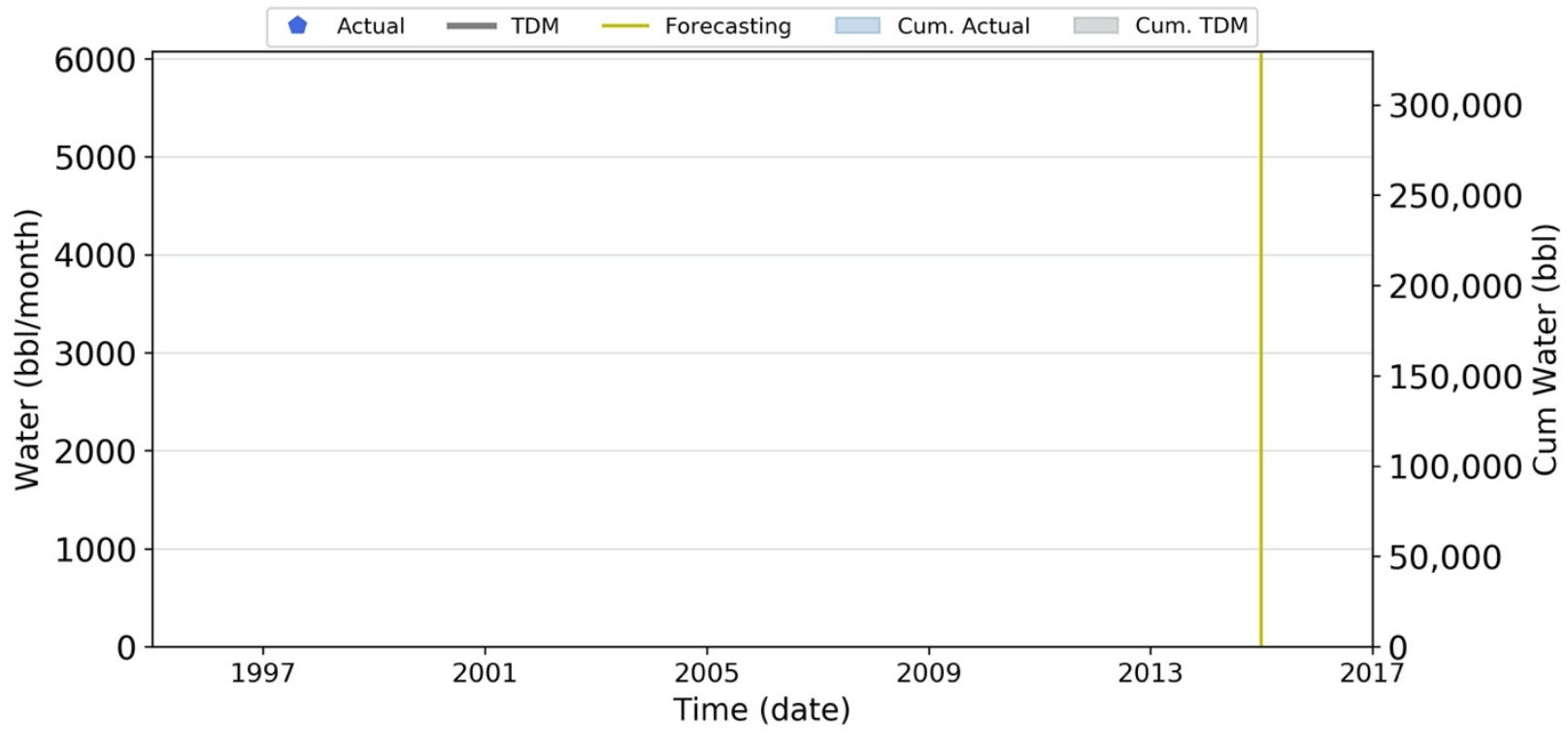




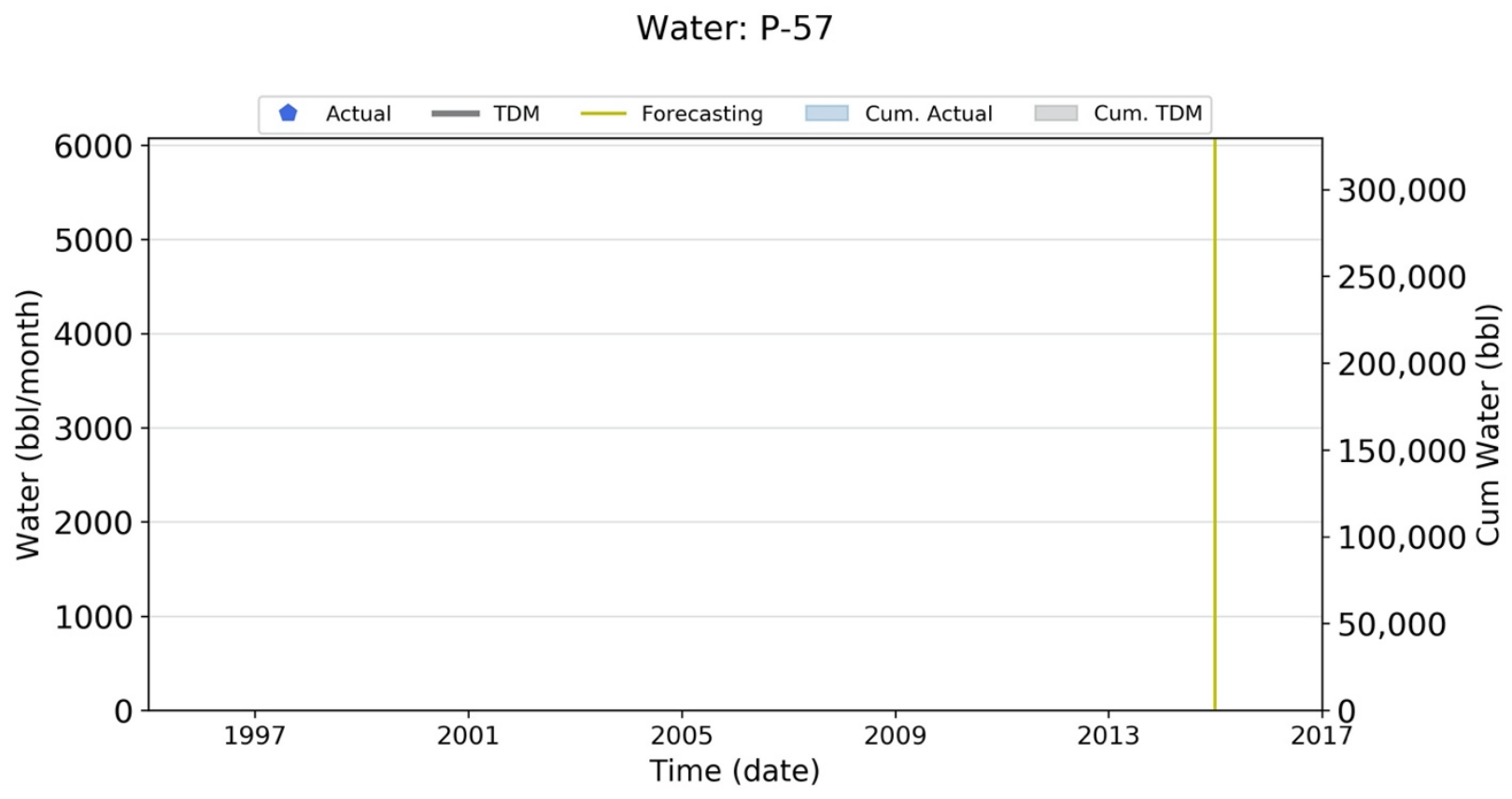


Reservoir Pressure (psi) at 2014-01-31
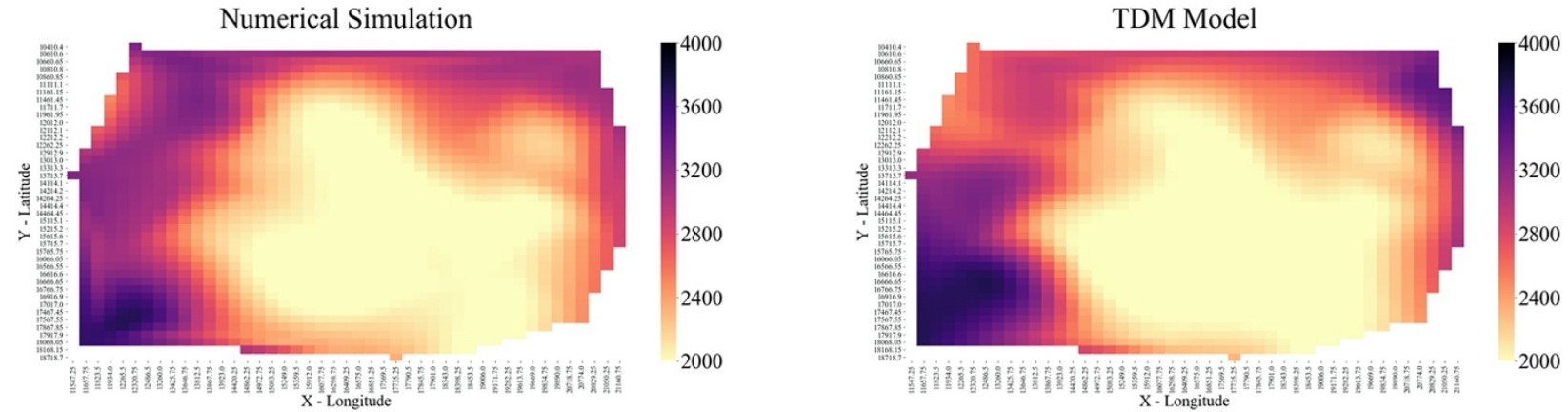

Reservoir Pressure (psi) at 2014-02-28
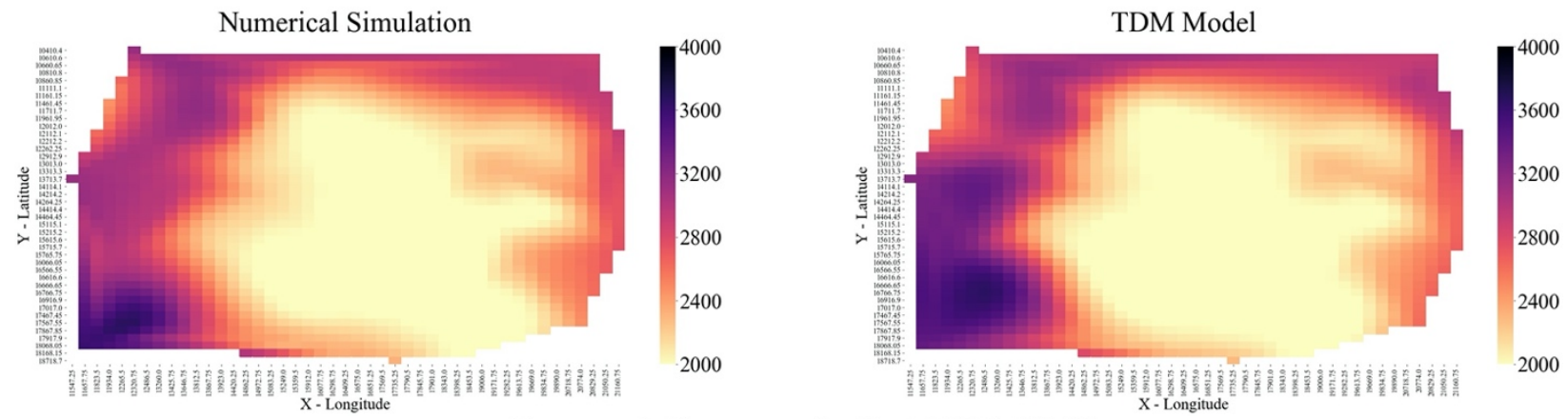

Reservoir Pressure (psi) at 2014-03-31
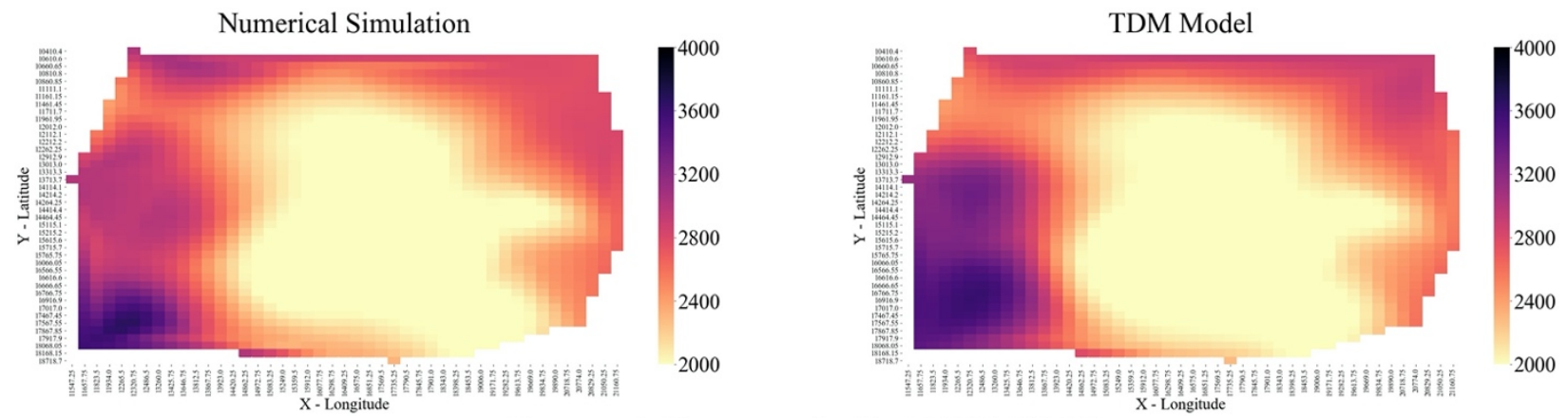

Reservoir Pressure (psi) at 2014-04-30
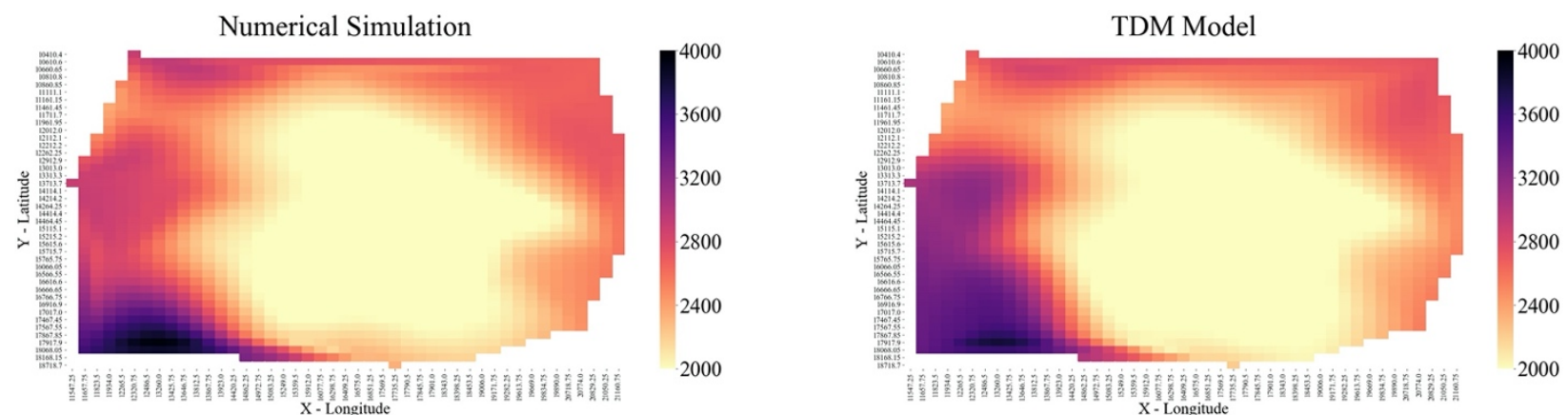
Reservoir Pressure (psi) at 2014-05-31
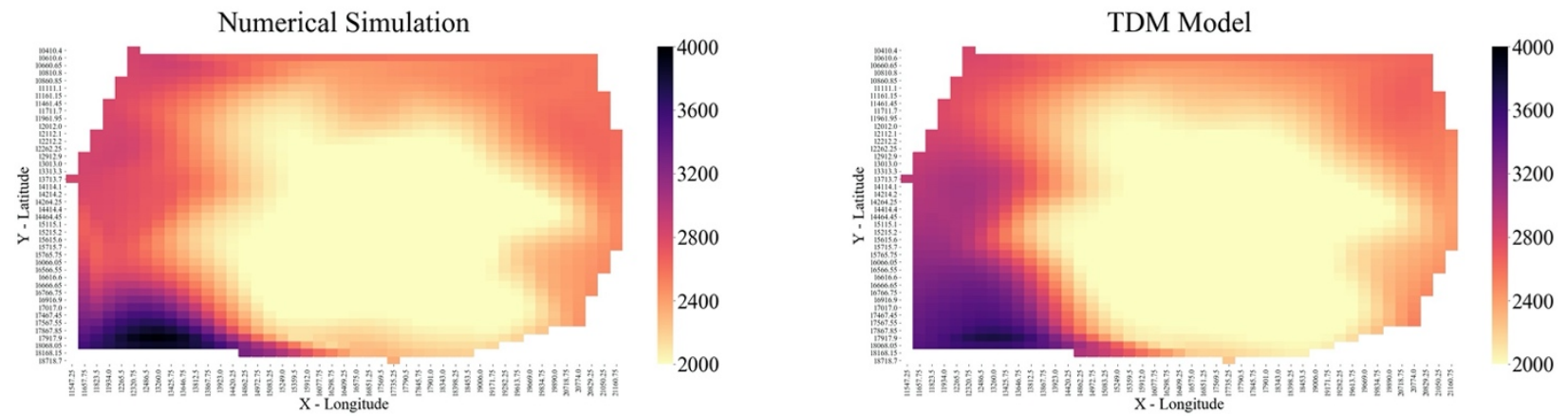

Reservoir Pressure (psi) at 2014-06-30
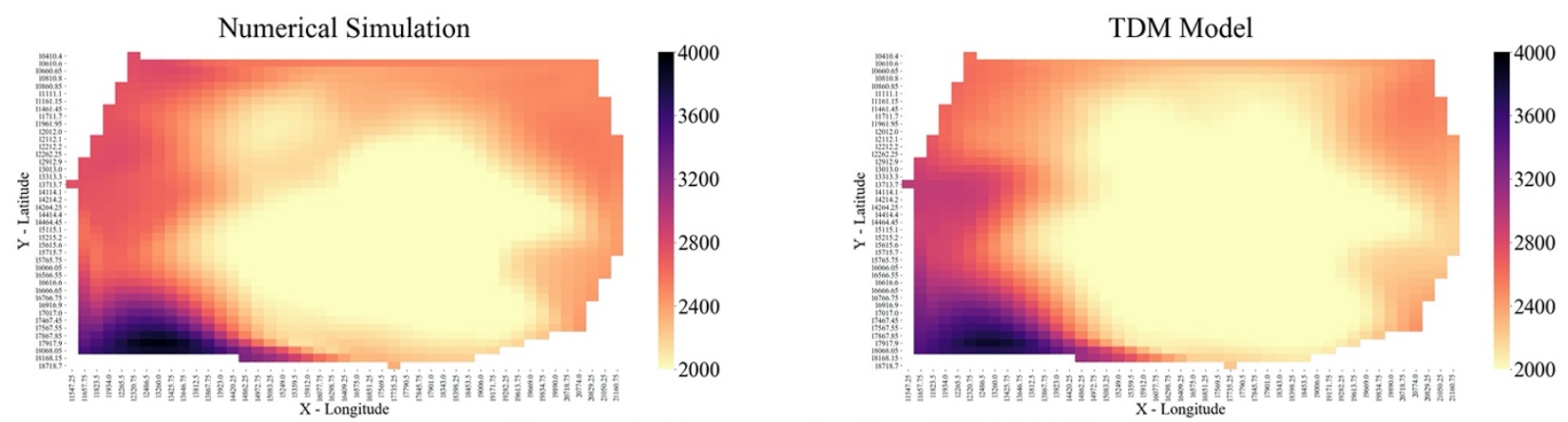

Reservoir Pressure (psi) at 2014-07-31
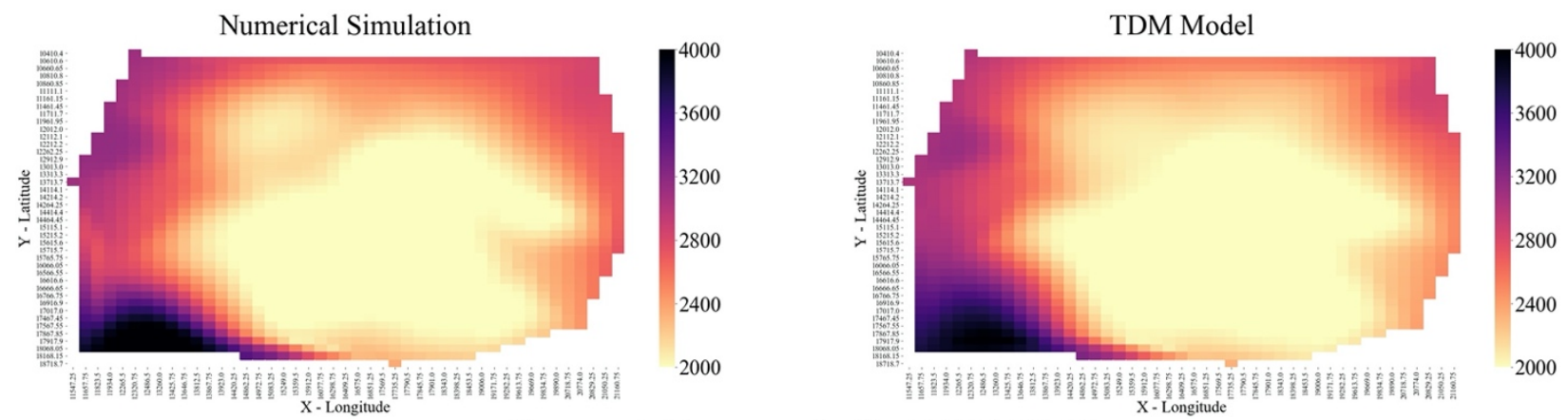

Reservoir Pressure (psi) at 2014-08-31
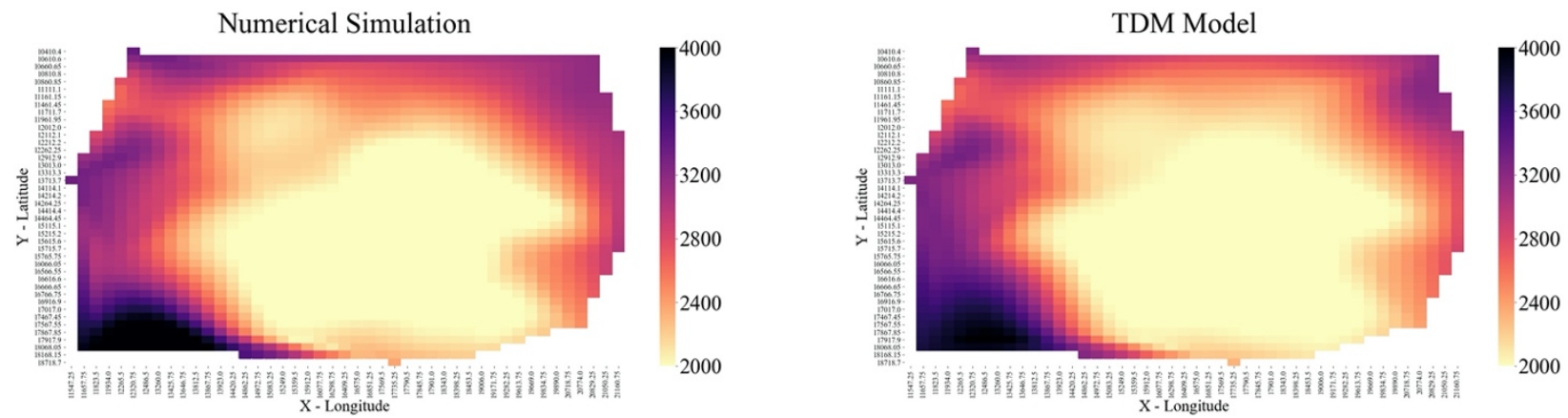
Reservoir Pressure (psi) at 2014-09-30
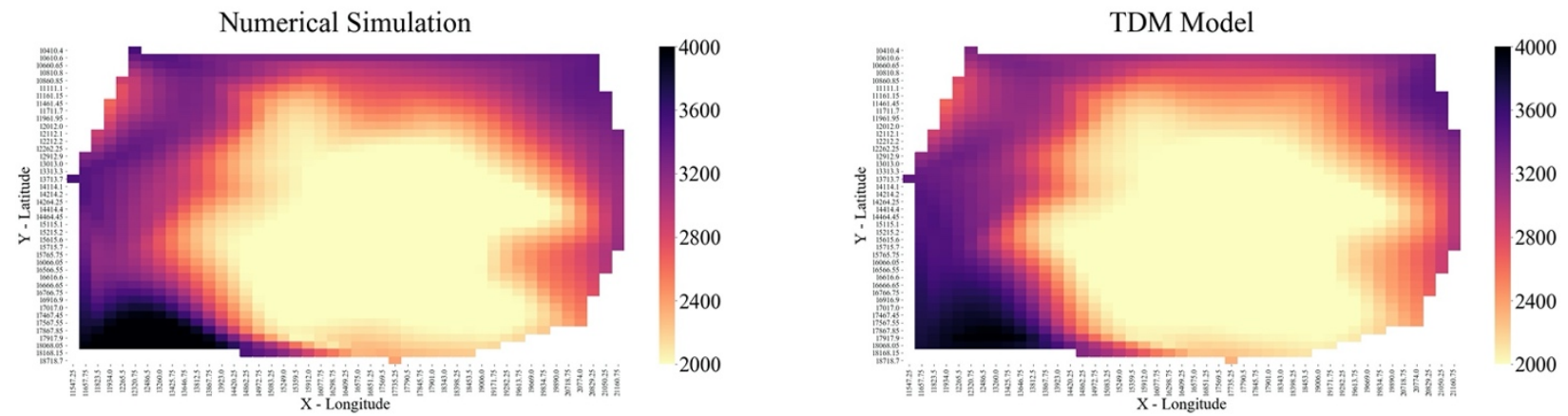

Reservoir Pressure (psi) at 2014-10-31
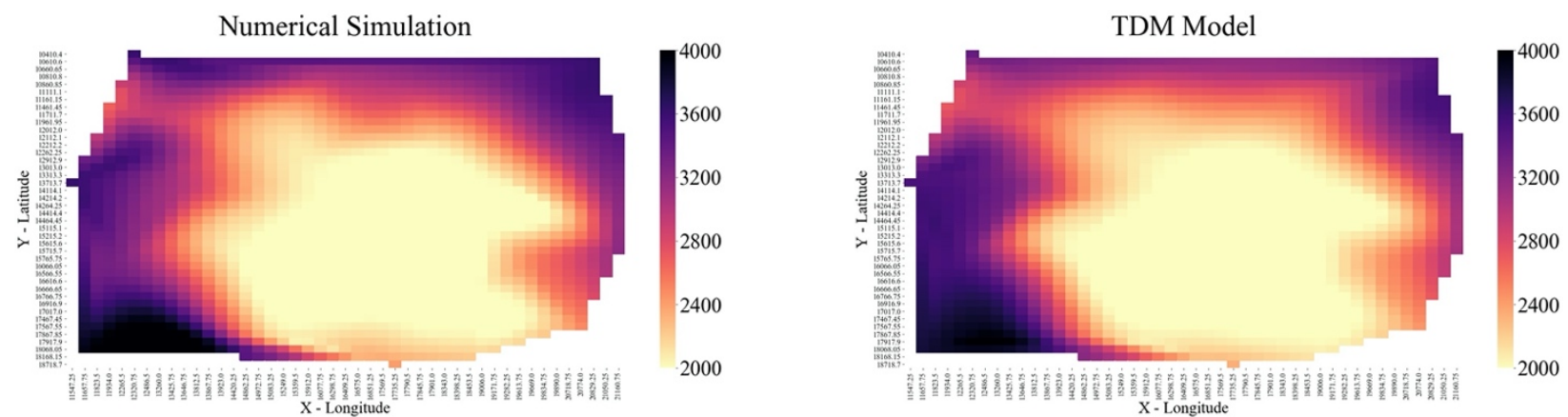

Reservoir Pressure (psi) at 2014-11-30
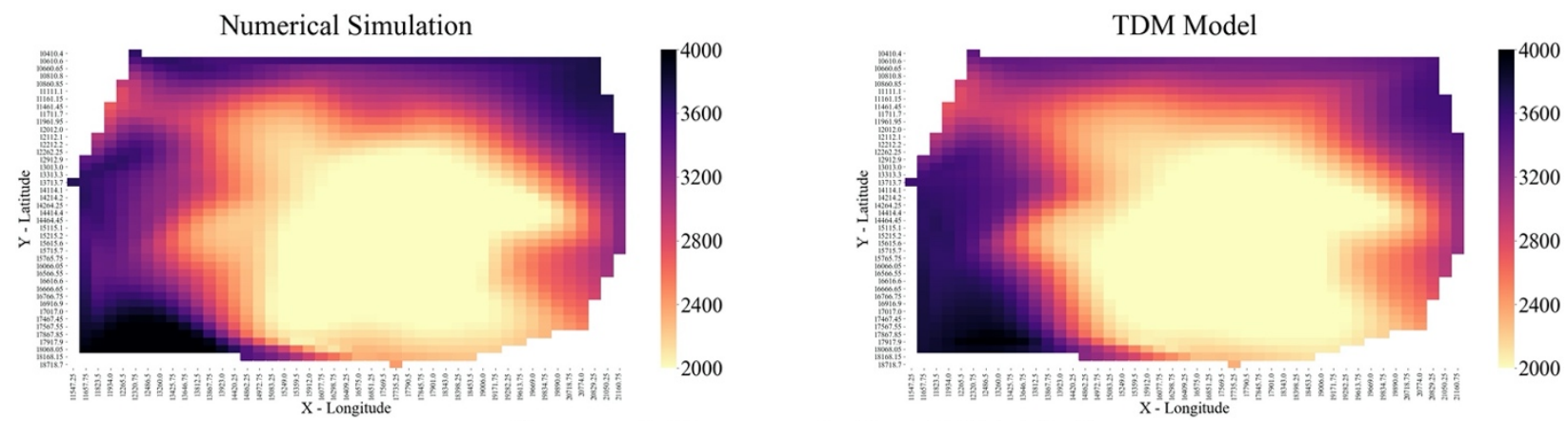

Reservoir Pressure (psi) at 2014-12-31
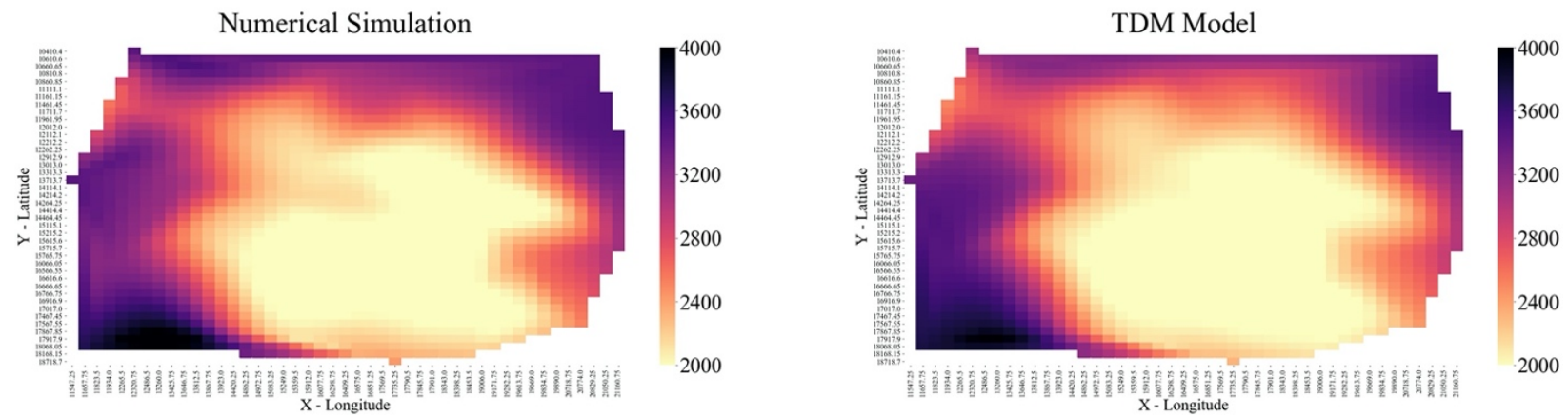
Reservoir Pressure (psi) at 2015-01-31
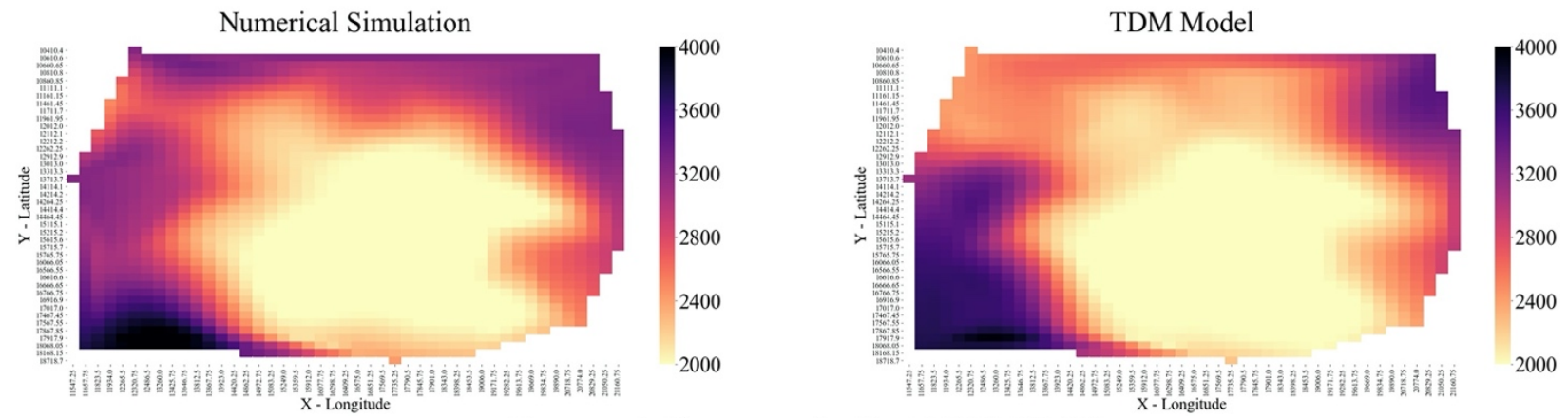

Reservoir Pressure (psi) at 2015-02-28
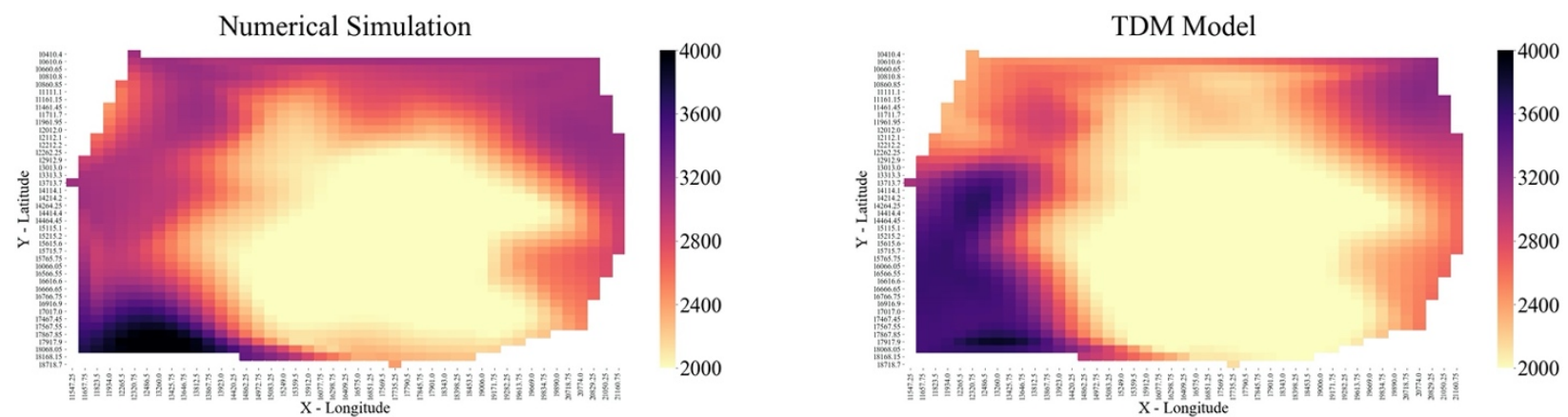

Reservoir Pressure (psi) at 2015-03-31
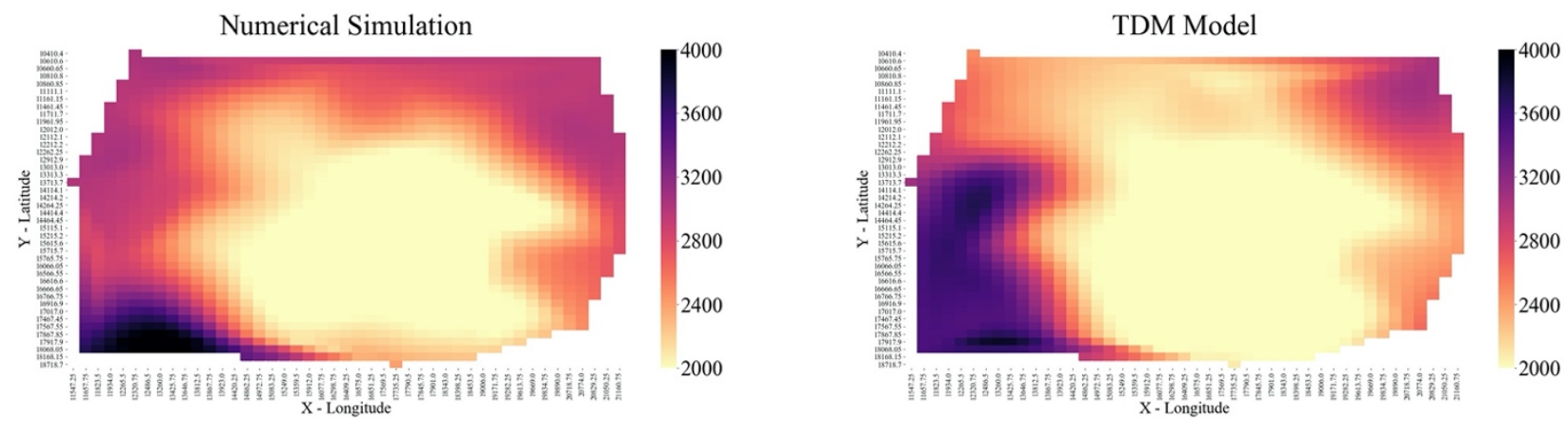

Reservoir Pressure (psi) at 2015-04-30
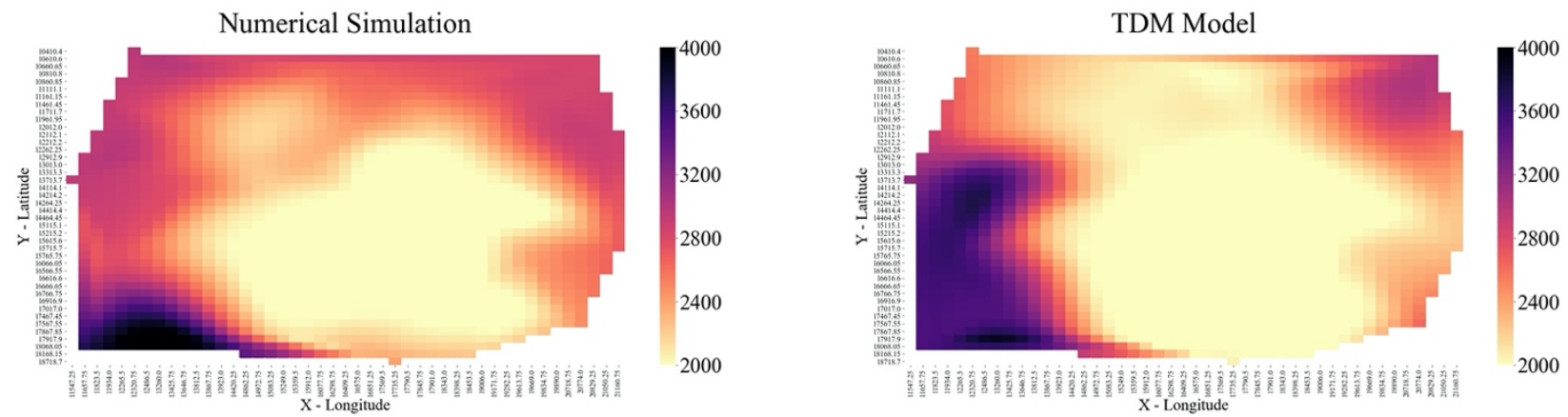
Reservoir Pressure (psi) at 2015-05-31
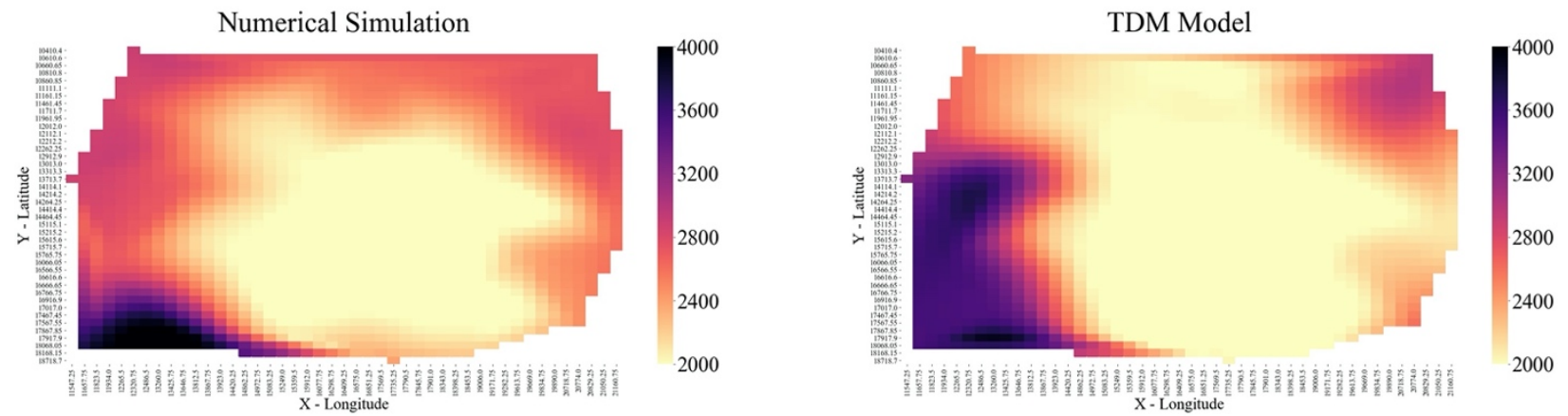

Reservoir Pressure (psi) at 2015-06-30
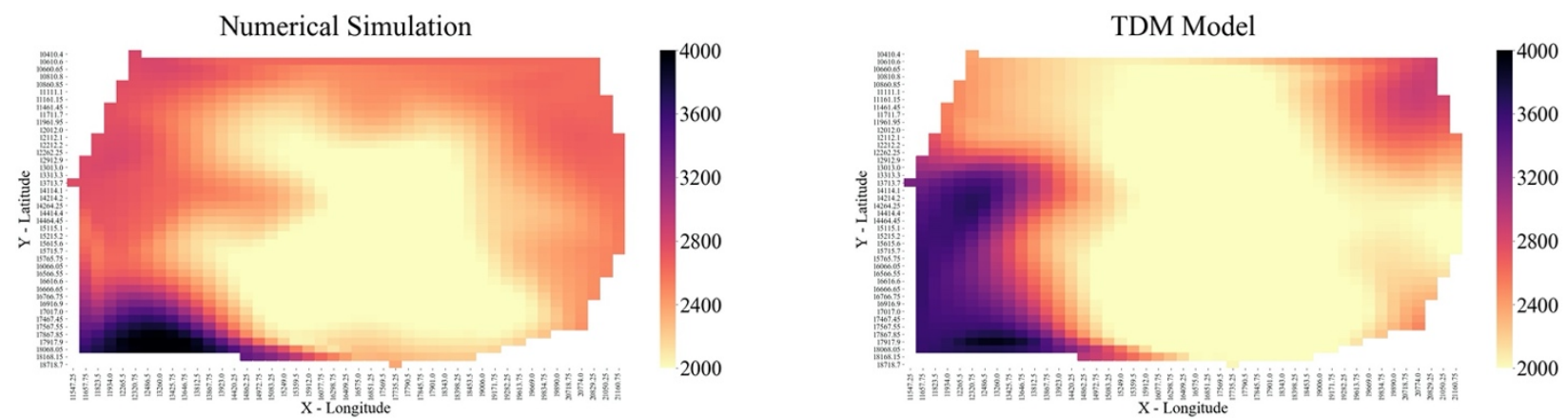

Reservoir Pressure (psi) at 2015-07-31
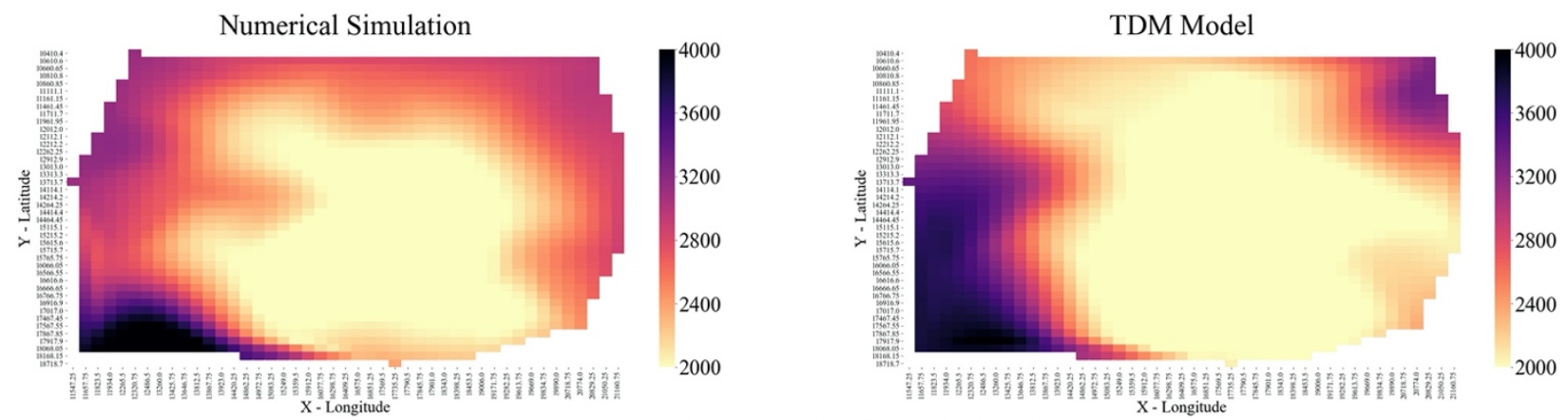

Reservoir Pressure (psi) at 2015-08-31
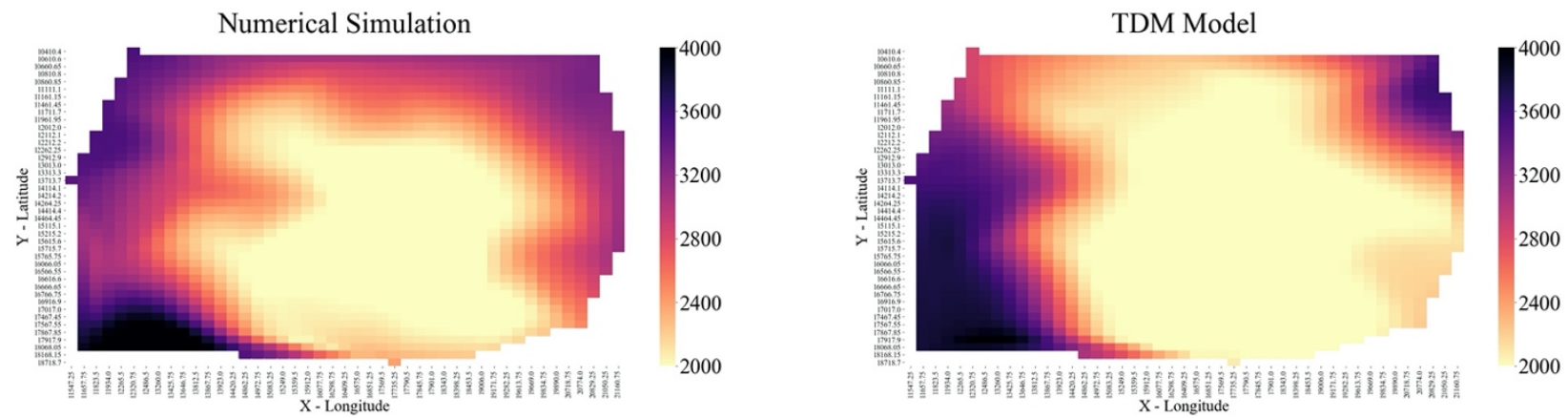
Reservoir Pressure (psi) at 2015-09-30
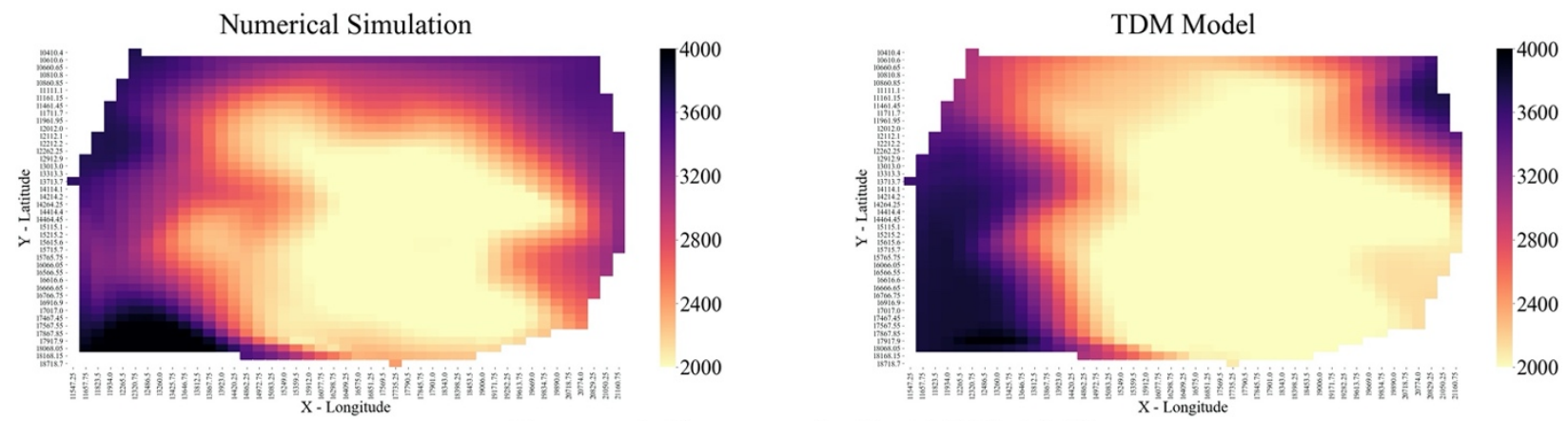

Reservoir Pressure (psi) at 2015-10-31
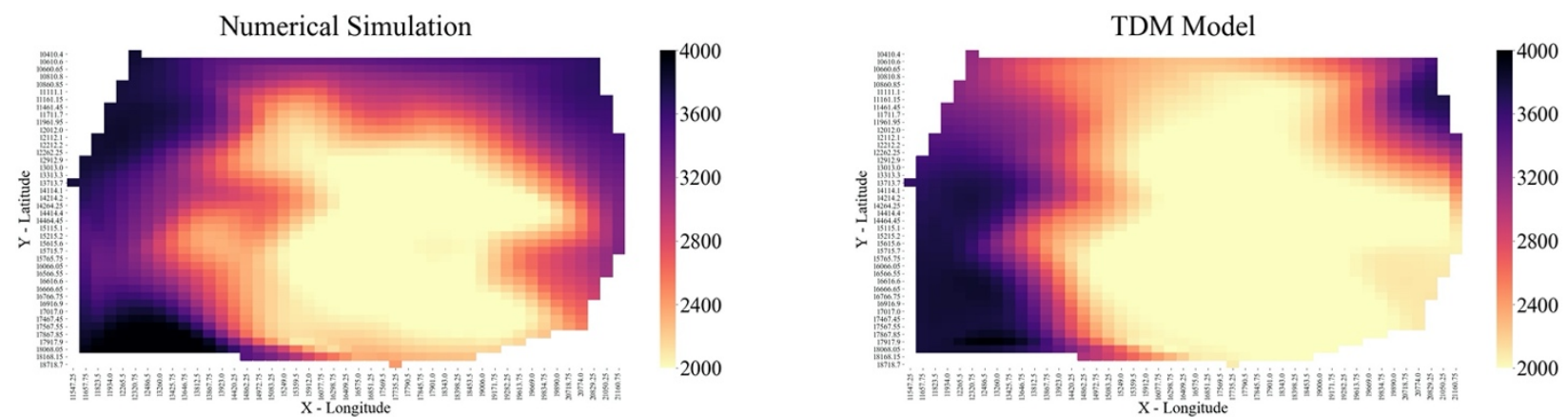

Reservoir Pressure (psi) at 2015-11-30
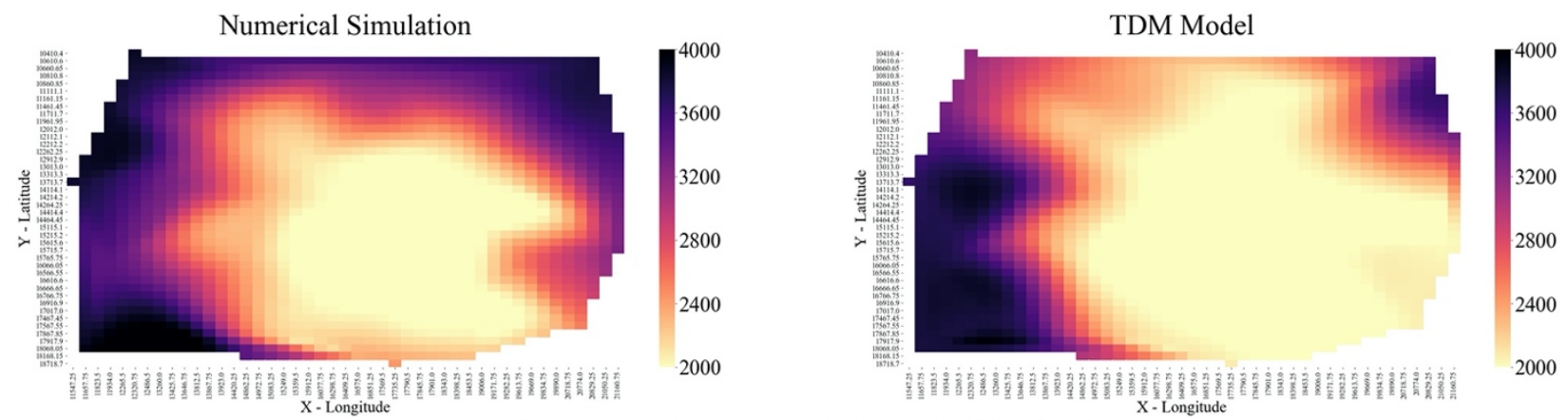

Reservoir Pressure (psi) at 2015-12-31
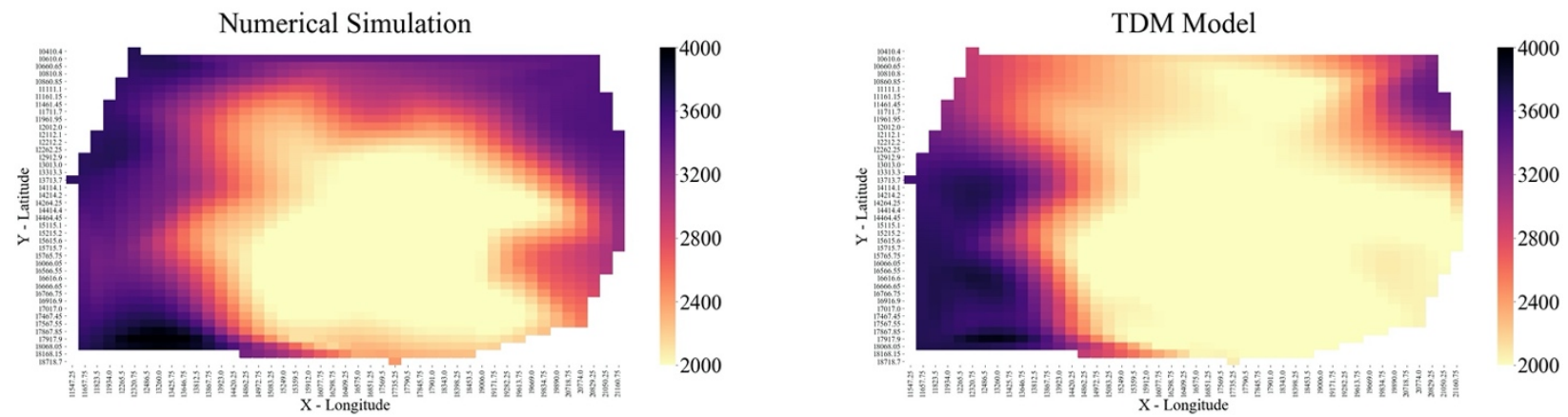
Reservoir Pressure (psi) at 2016-01-31
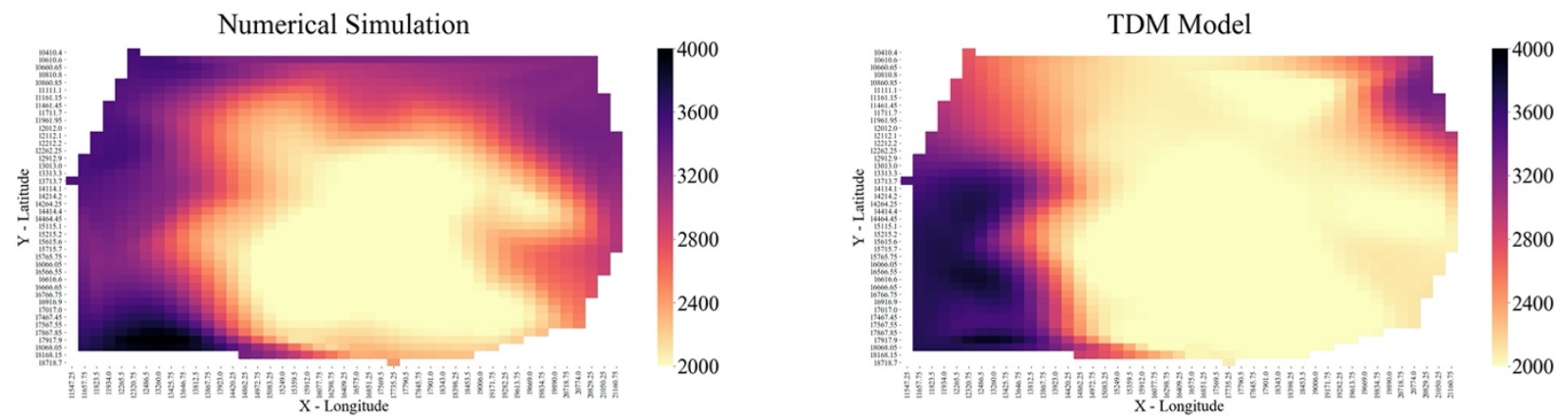

Reservoir Pressure (psi) at 2016-02-29
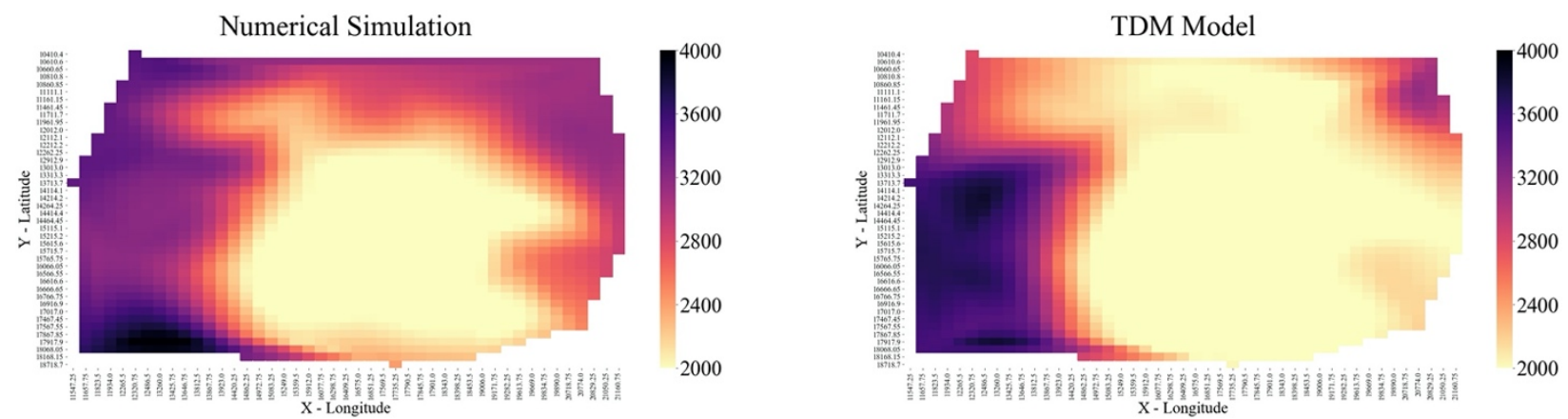

Reservoir Pressure (psi) at 2016-03-31
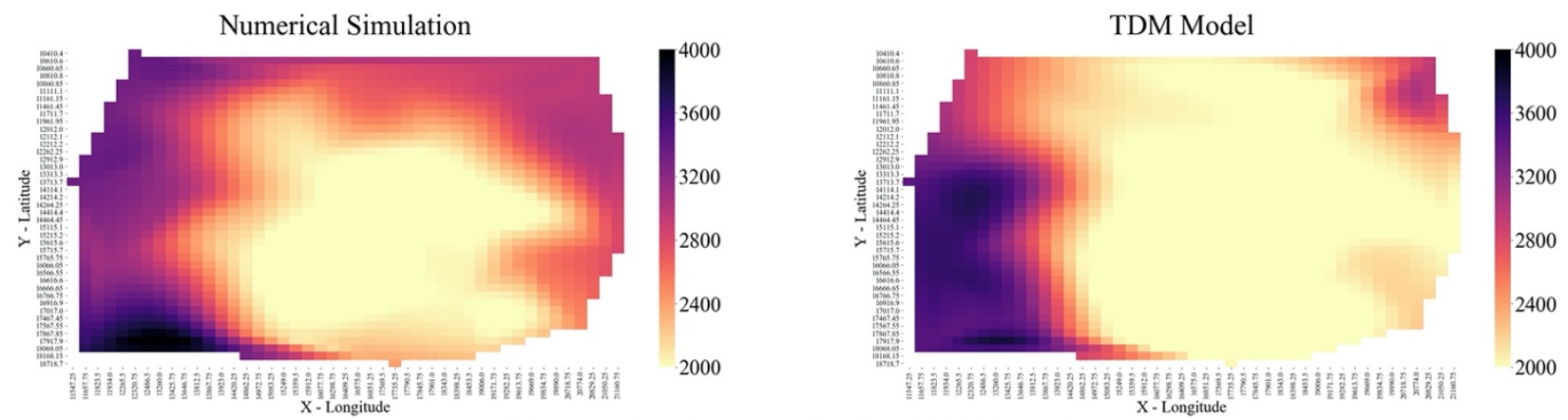

Reservoir Pressure (psi) at 2016-04-30
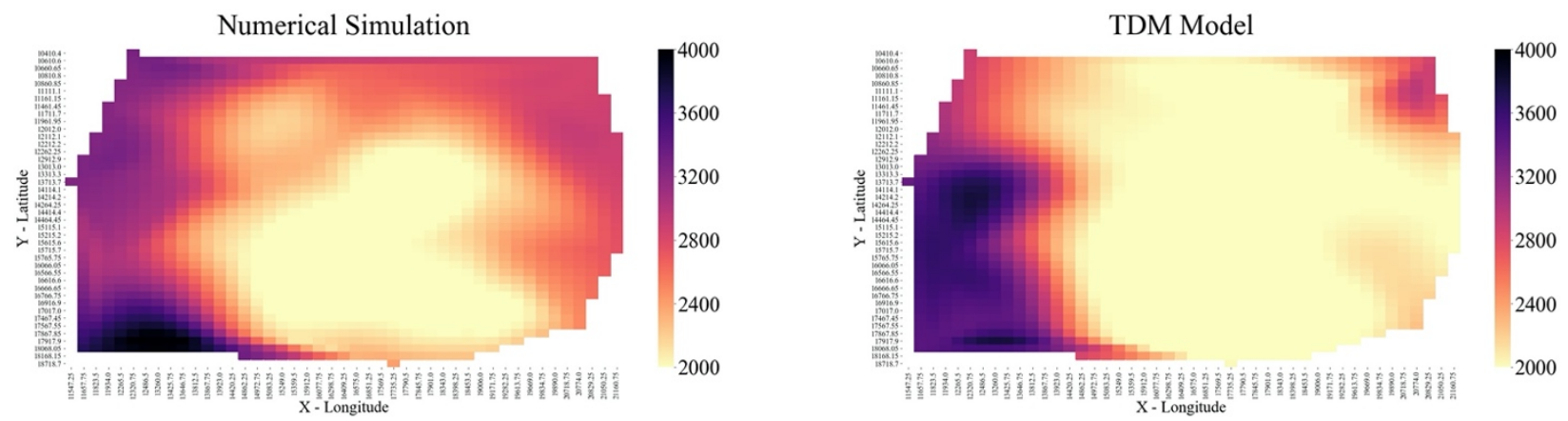
Reservoir Pressure (psi) at 2016-05-31
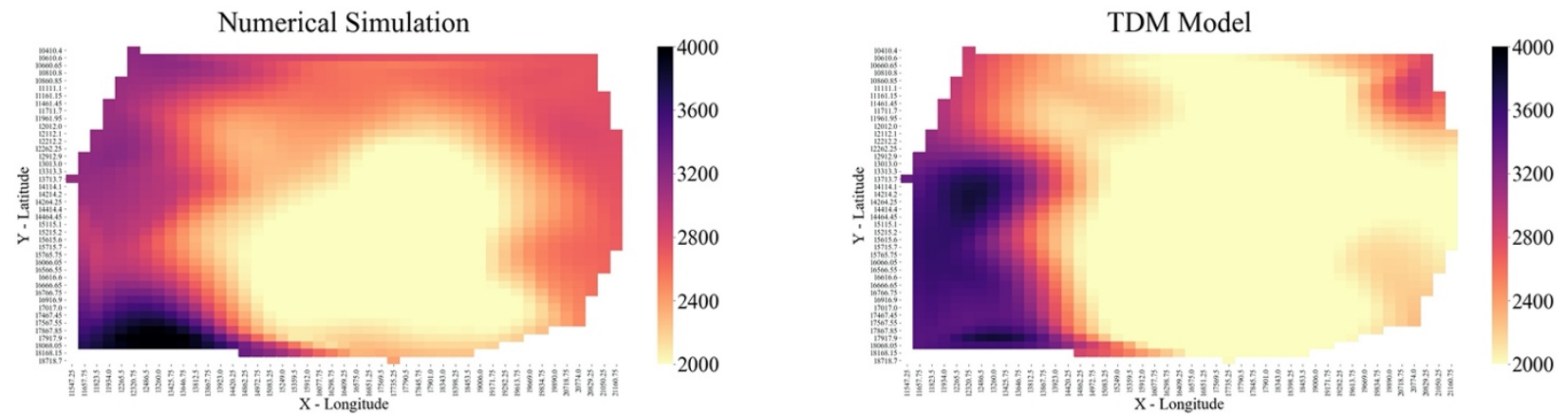

Reservoir Pressure (psi) at 2016-06-30
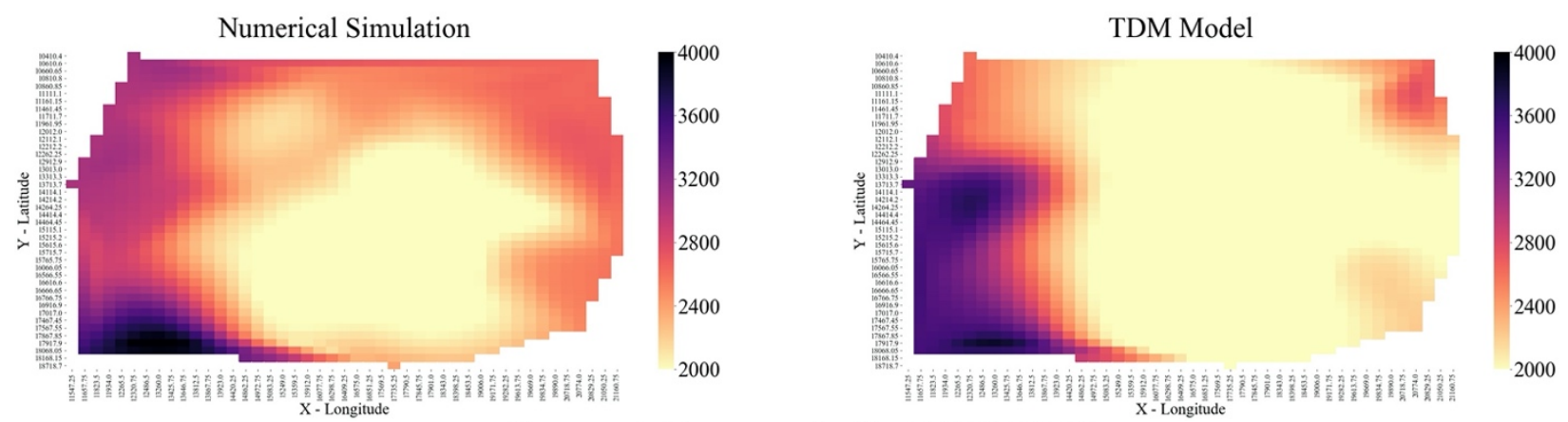

Reservoir Pressure (psi) at 2016-07-31
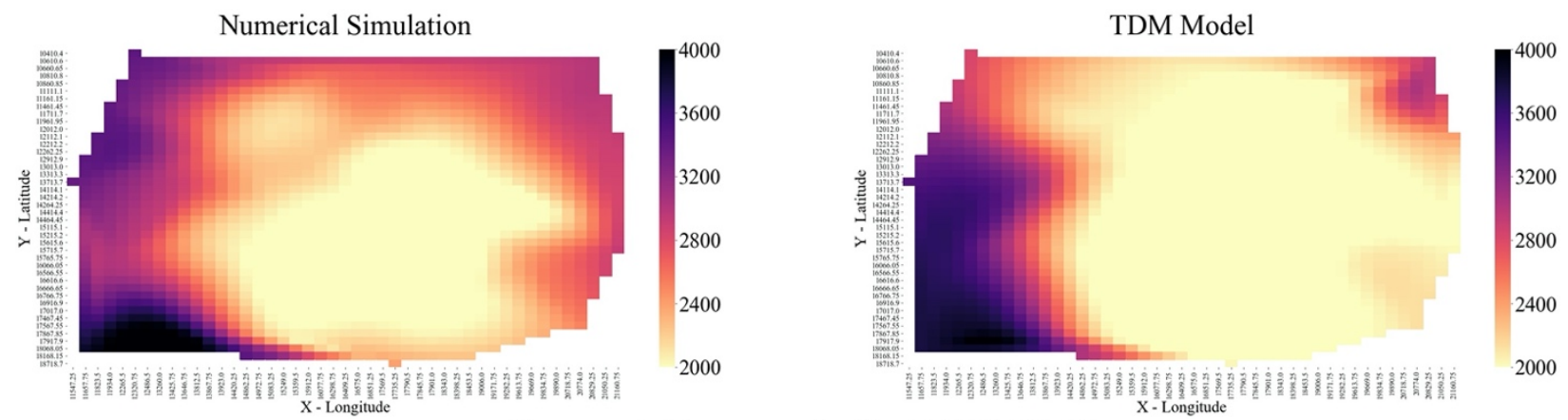

Reservoir Pressure (psi) at 2016-08-31
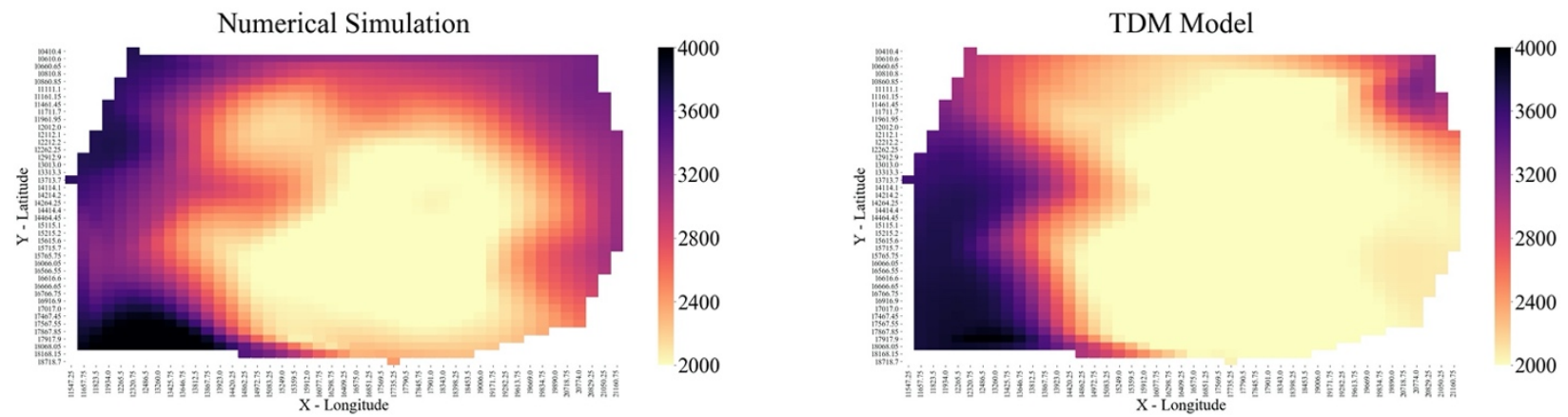
Reservoir Pressure (psi) at 2016-09-30
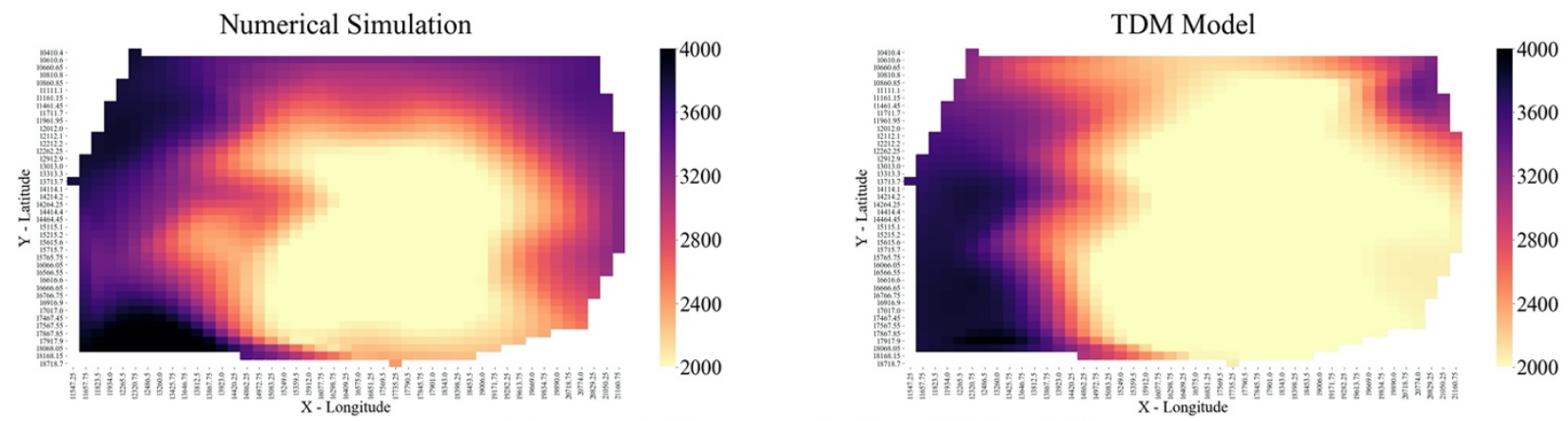

Reservoir Pressure (psi) at 2016-10-31
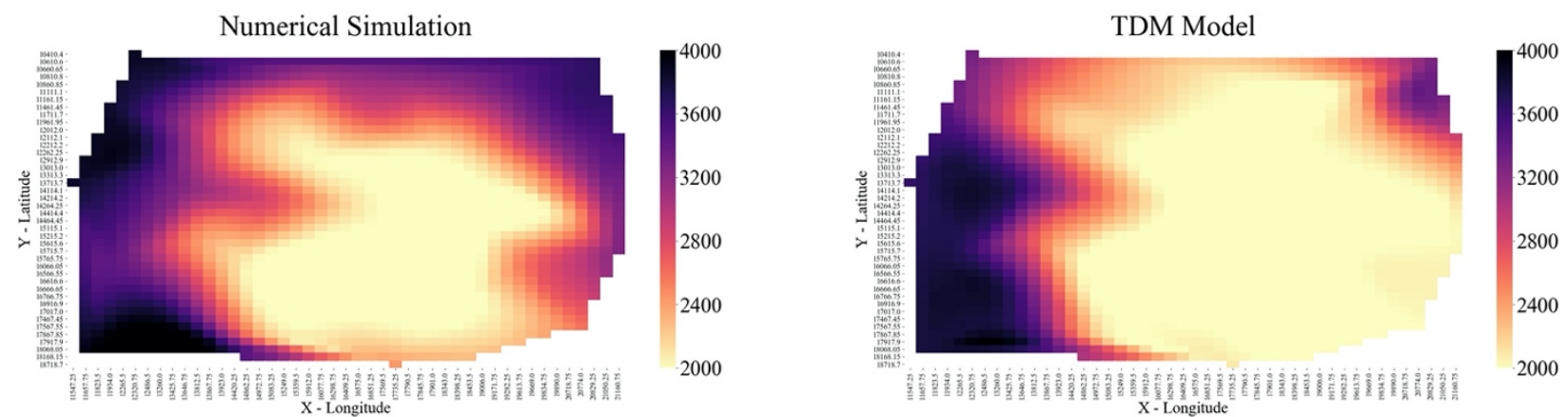

Reservoir Pressure (psi) at 2016-11-30
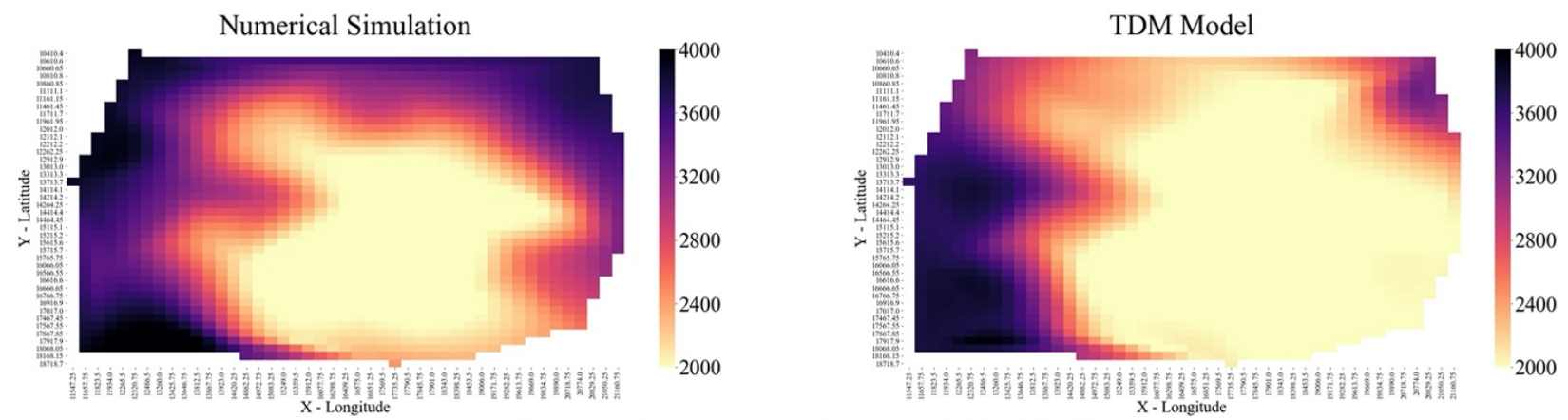

Reservoir Pressure (psi) at 2016-12-31
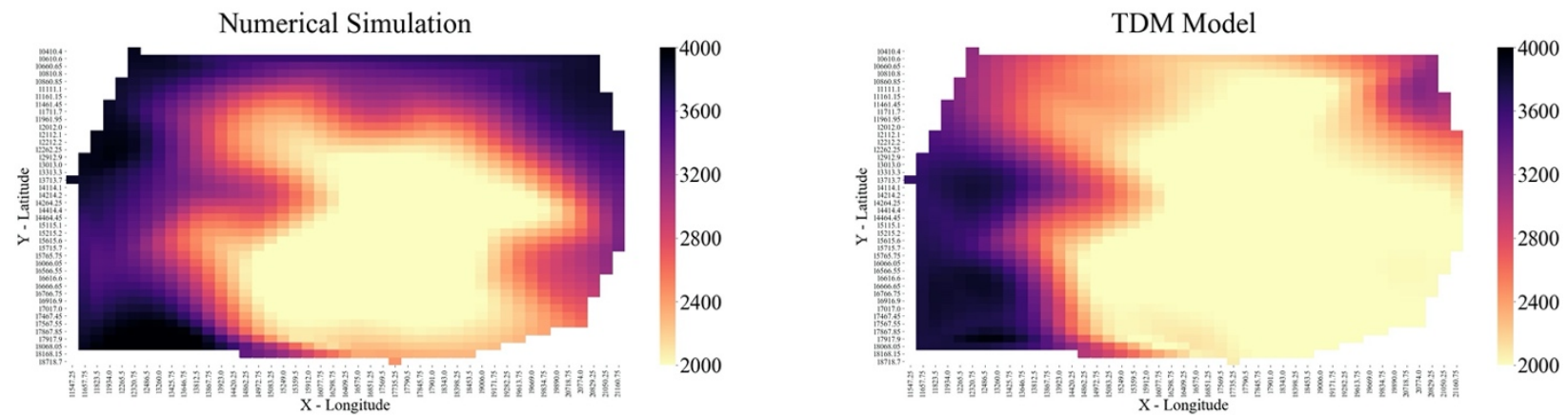
Saturation of Water Results

Water Saturation (\%) at 2014-01-31
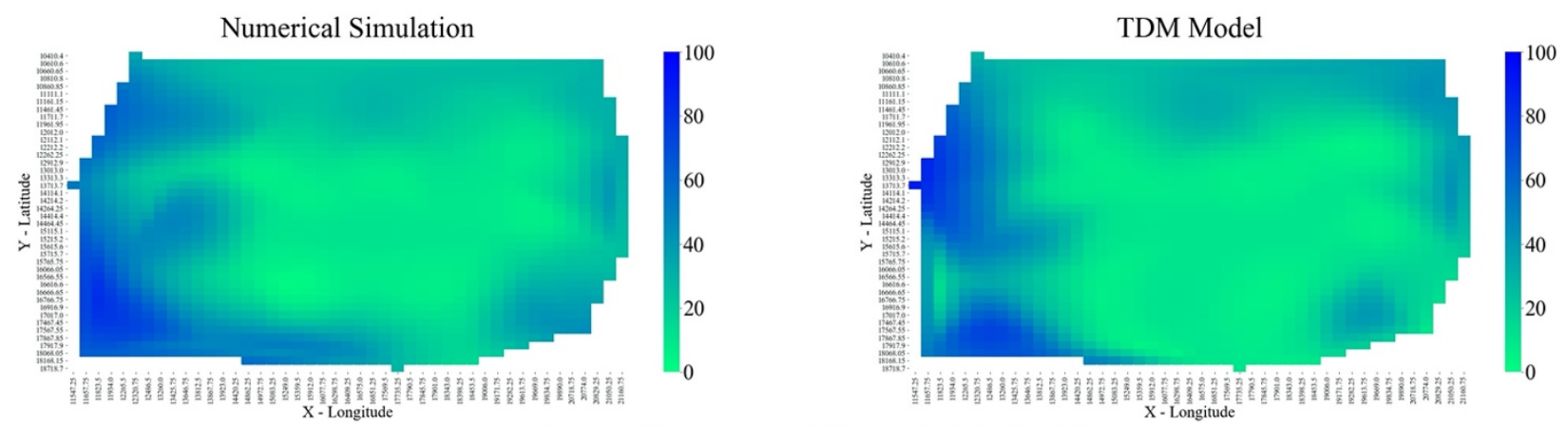

Water Saturation (\%) at 2014-02-28
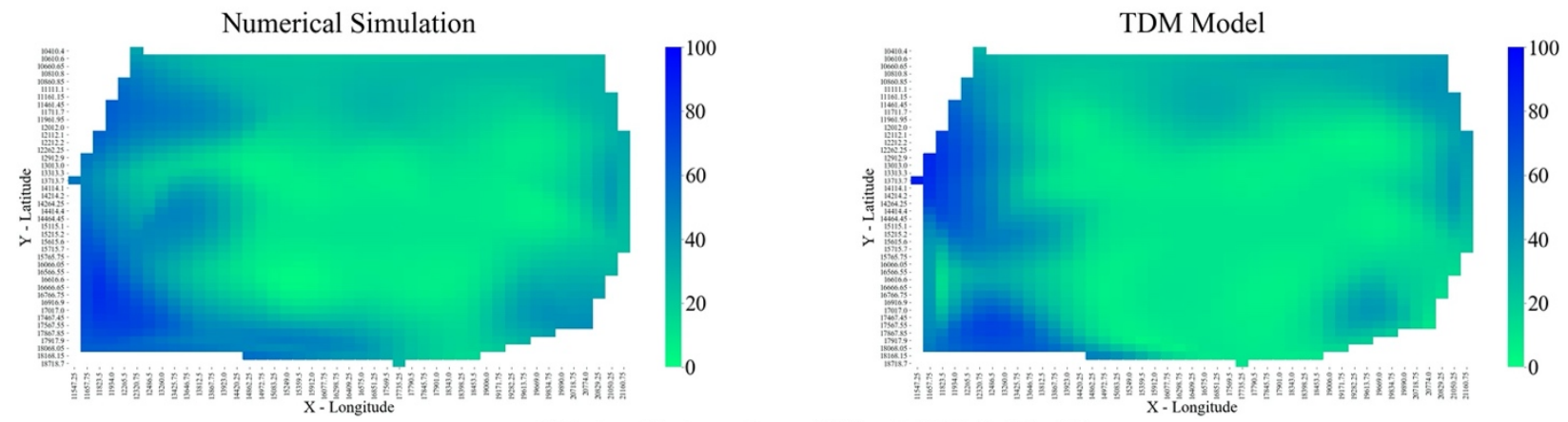

Water Saturation (\%) at 2014-03-31
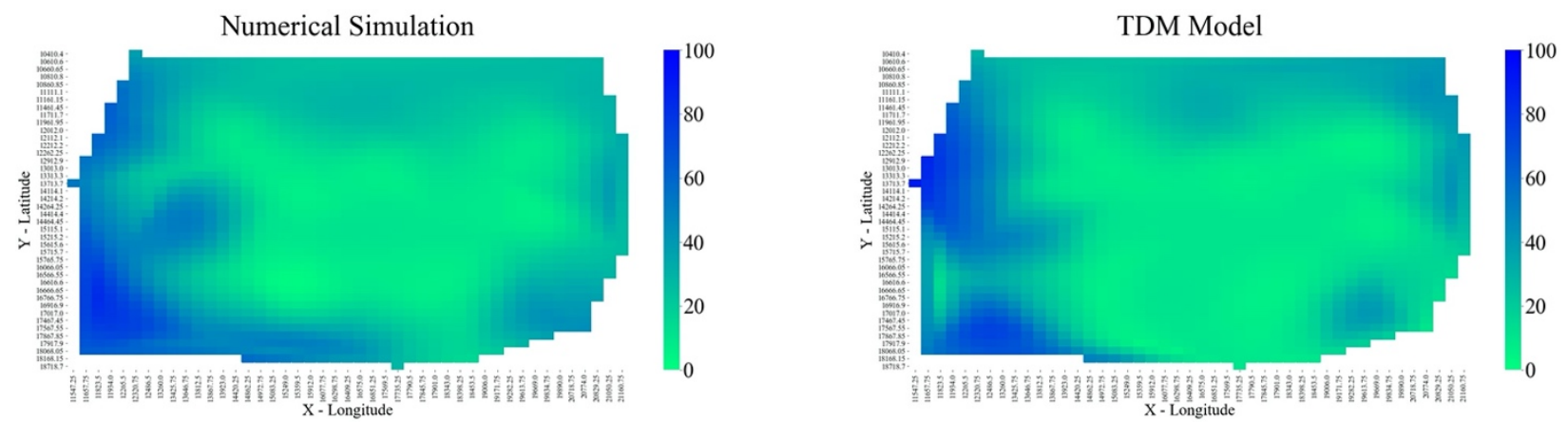

Water Saturation (\%) at 2014-04-30
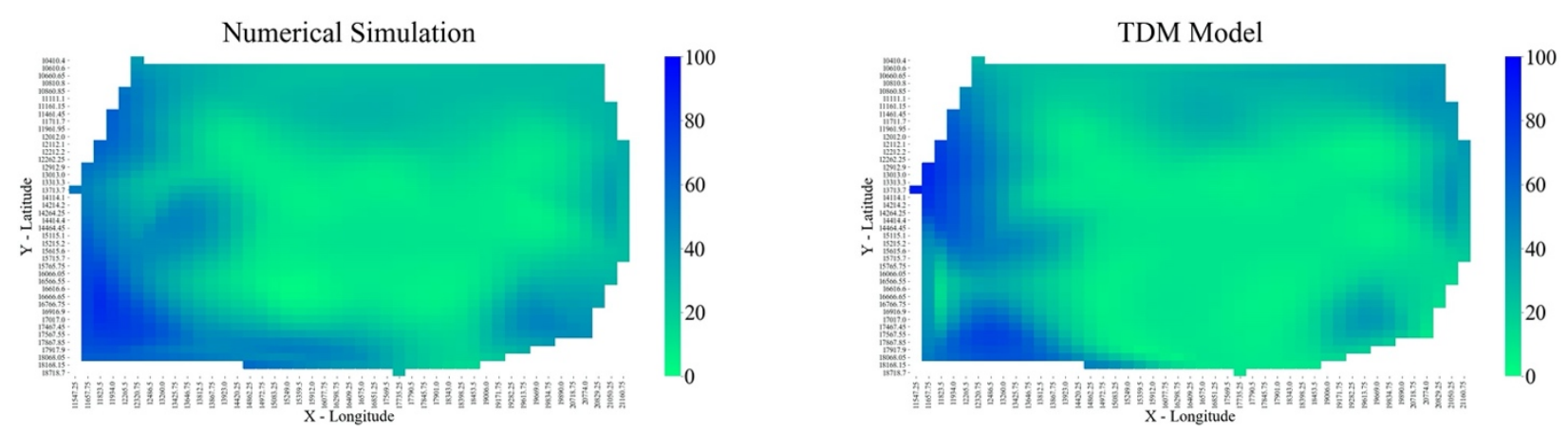
Water Saturation (\%) at 2014-05-31
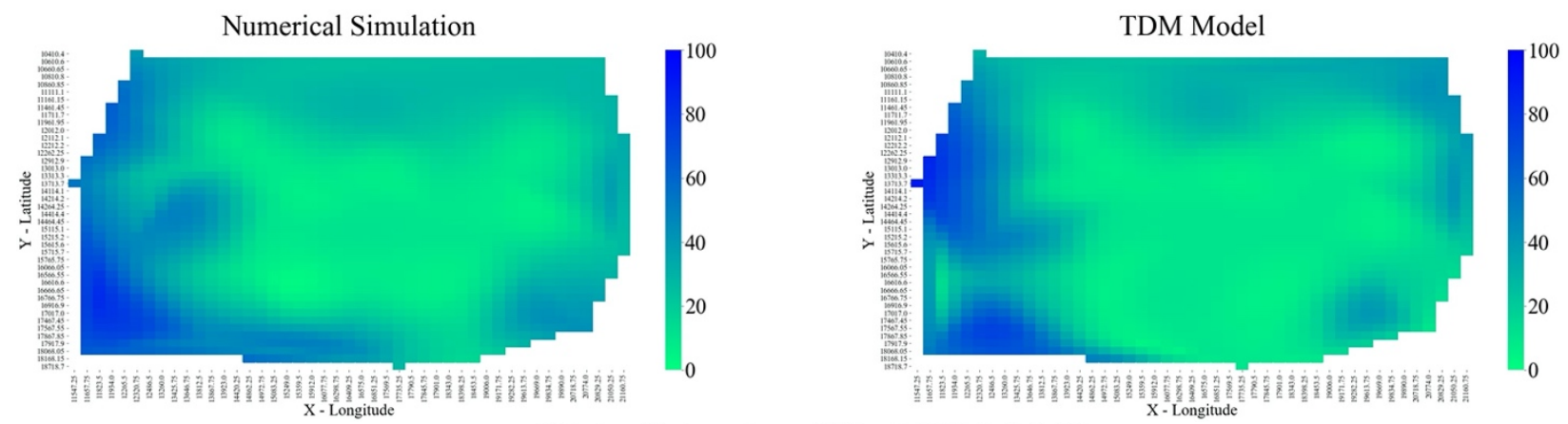

Water Saturation (\%) at 2014-06-30
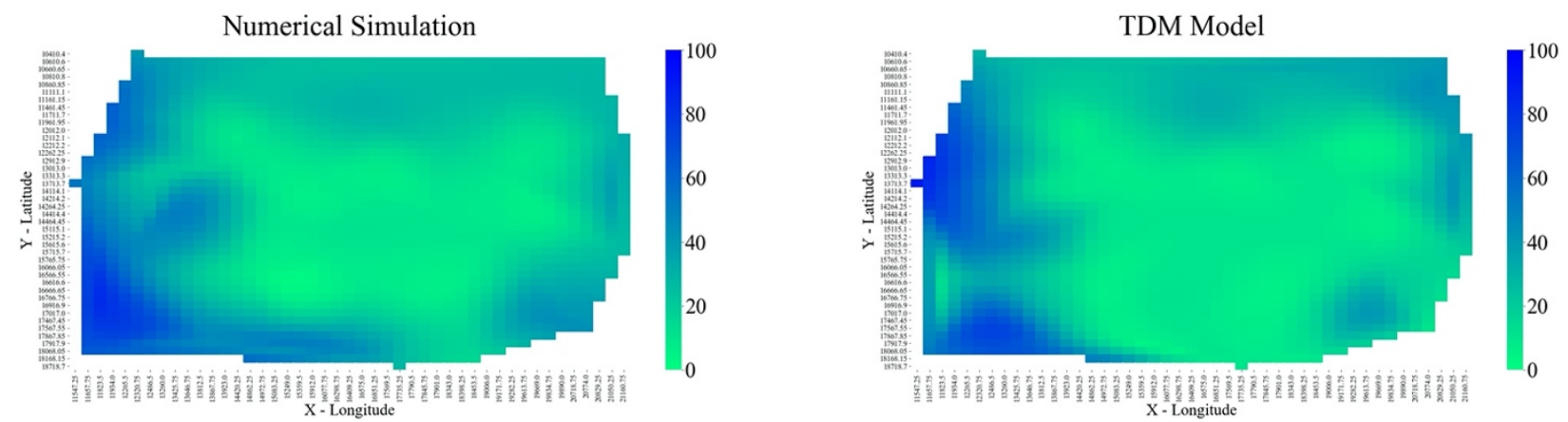

Water Saturation (\%) at 2014-07-31
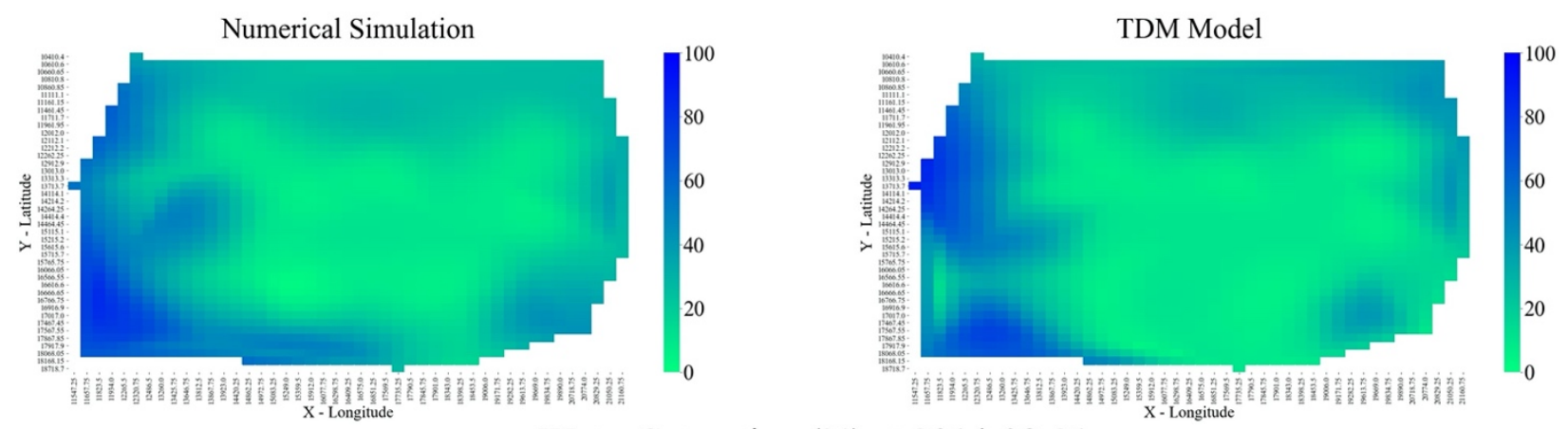

Water Saturation (\%) at 2014-08-31
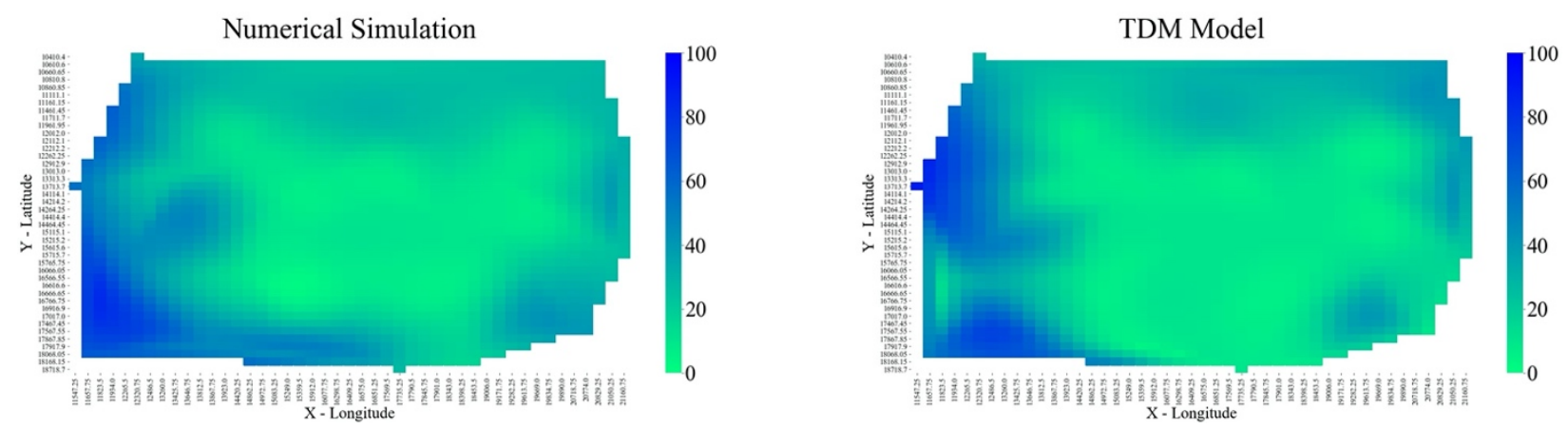
Water Saturation (\%) at 2014-09-30
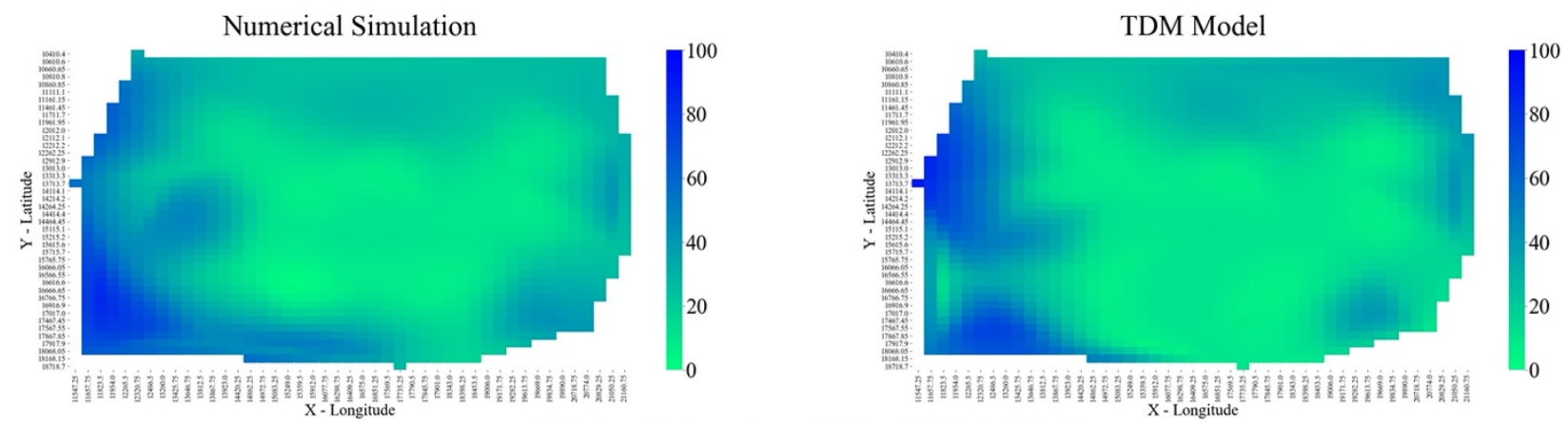

Water Saturation (\%) at 2014-10-31
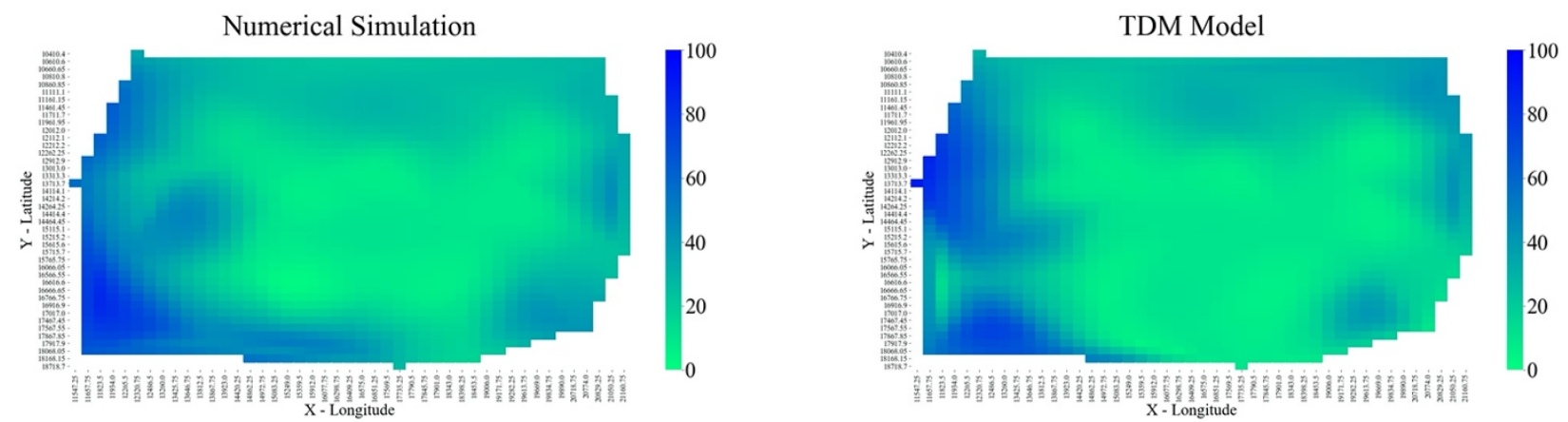

Water Saturation (\%) at 2014-11-30
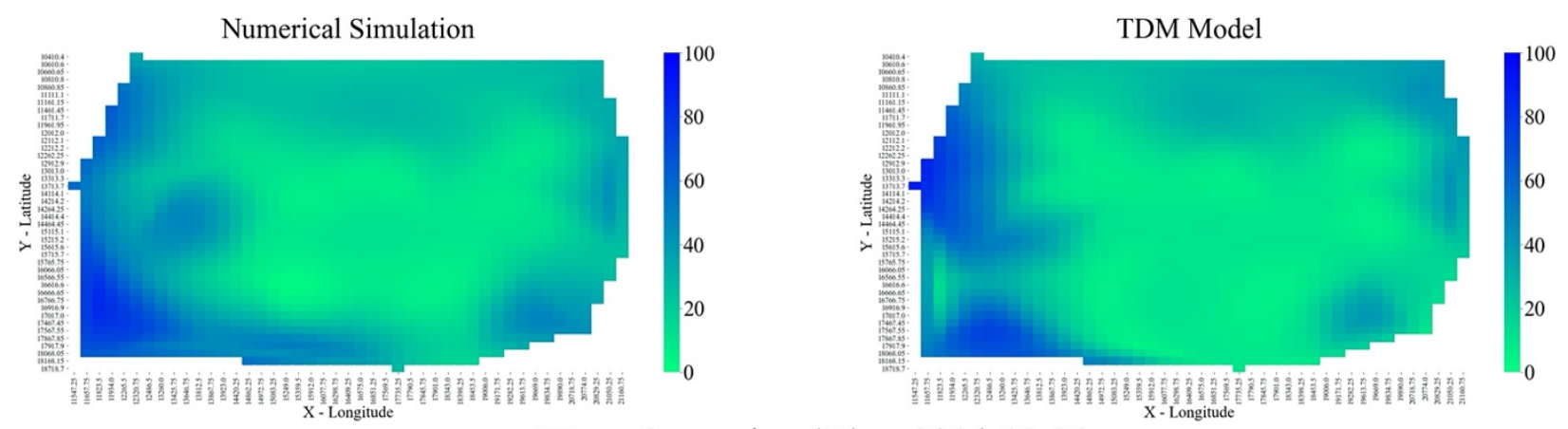

Water Saturation (\%) at 2014-12-31
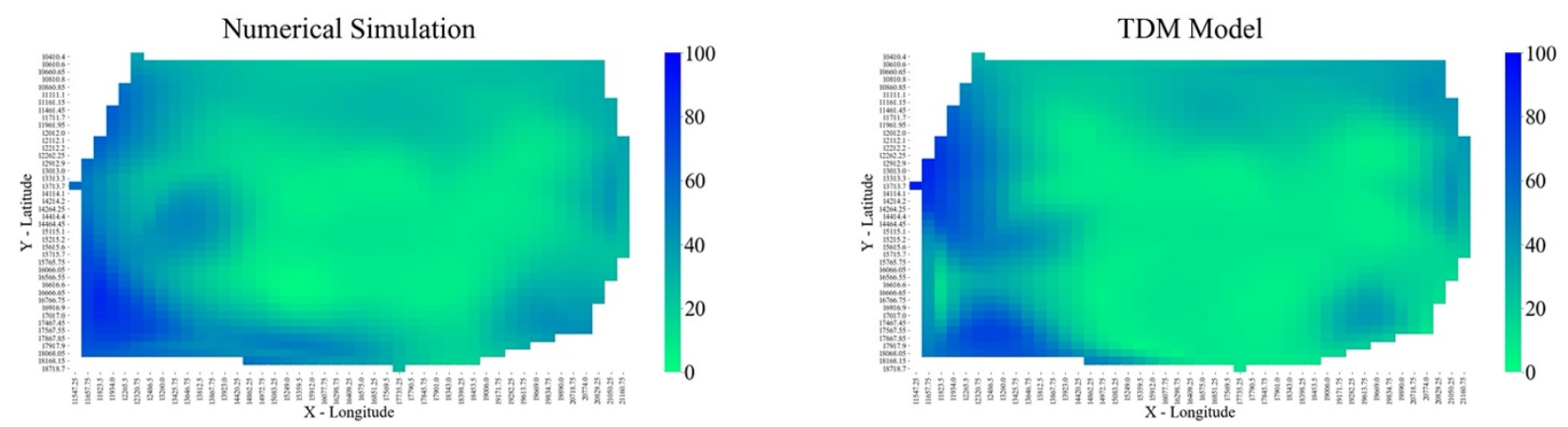
Water Saturation (\%) at 2015-01-31
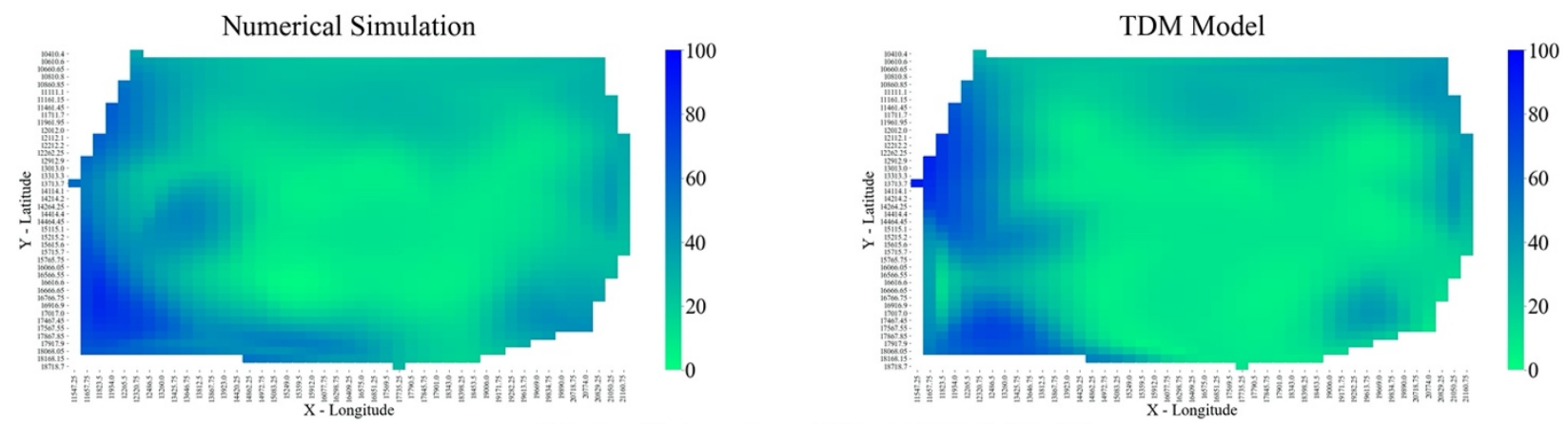

Water Saturation (\%) at 2015-02-28
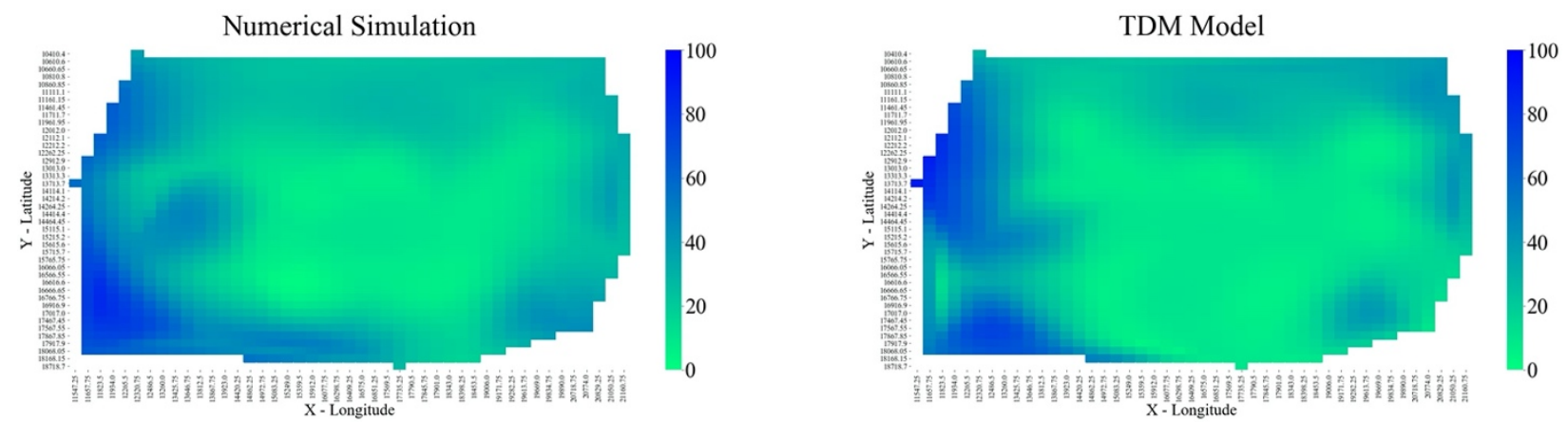

Water Saturation (\%) at 2015-03-31
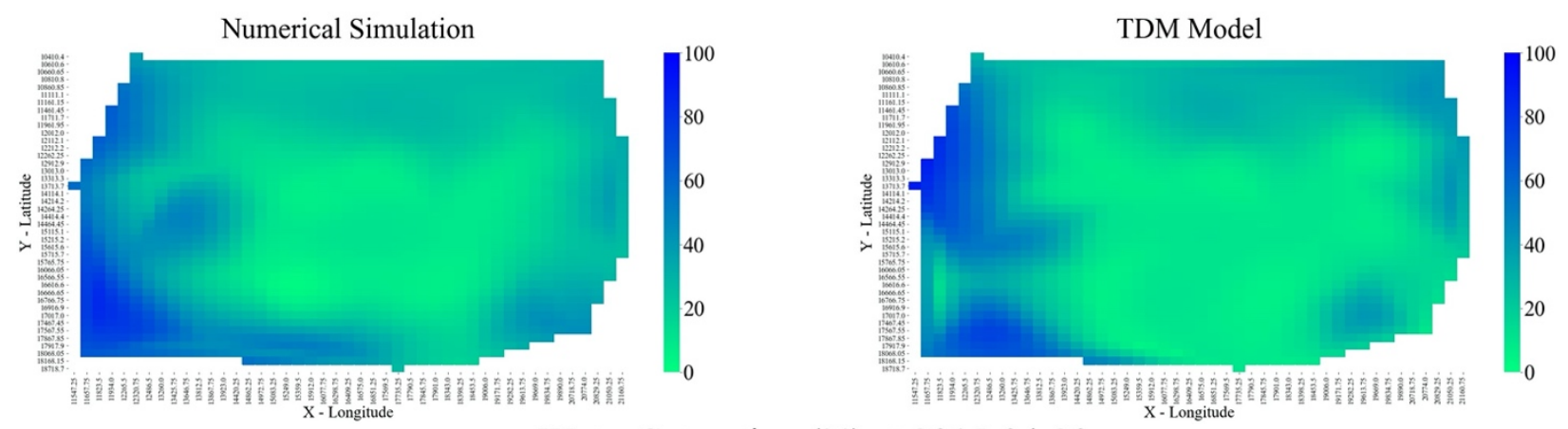

Water Saturation (\%) at 2015-04-30
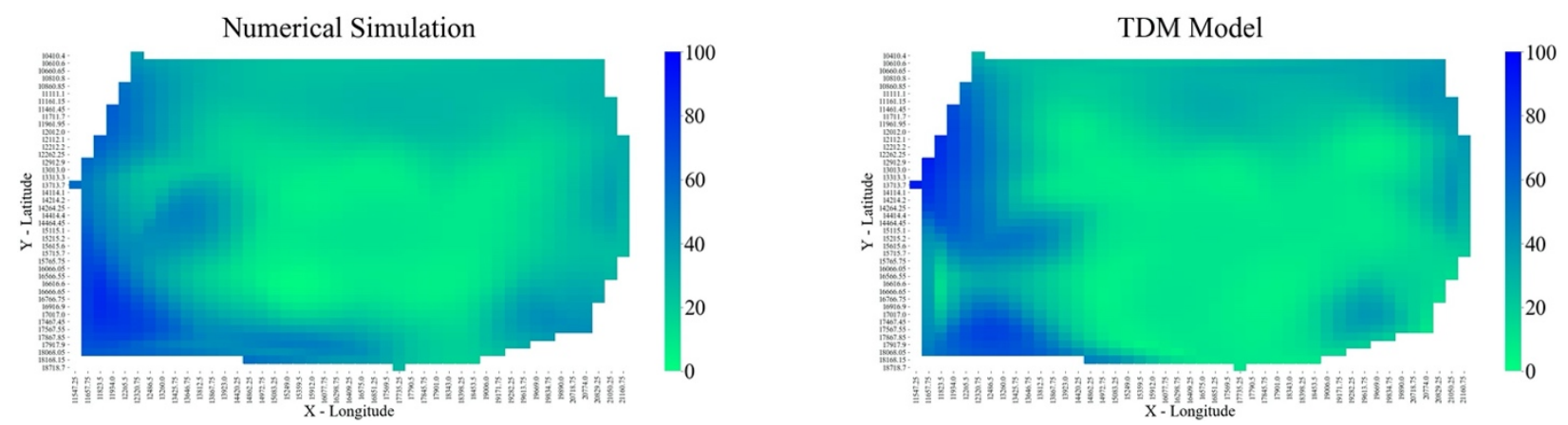
Water Saturation (\%) at 2015-05-31
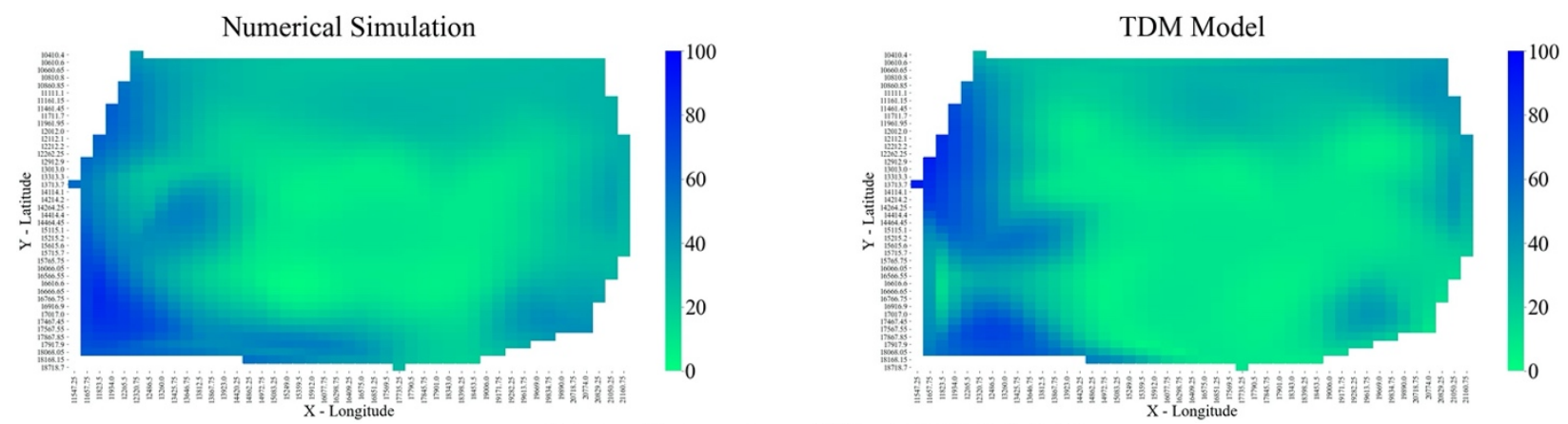

Water Saturation (\%) at 2015-06-30
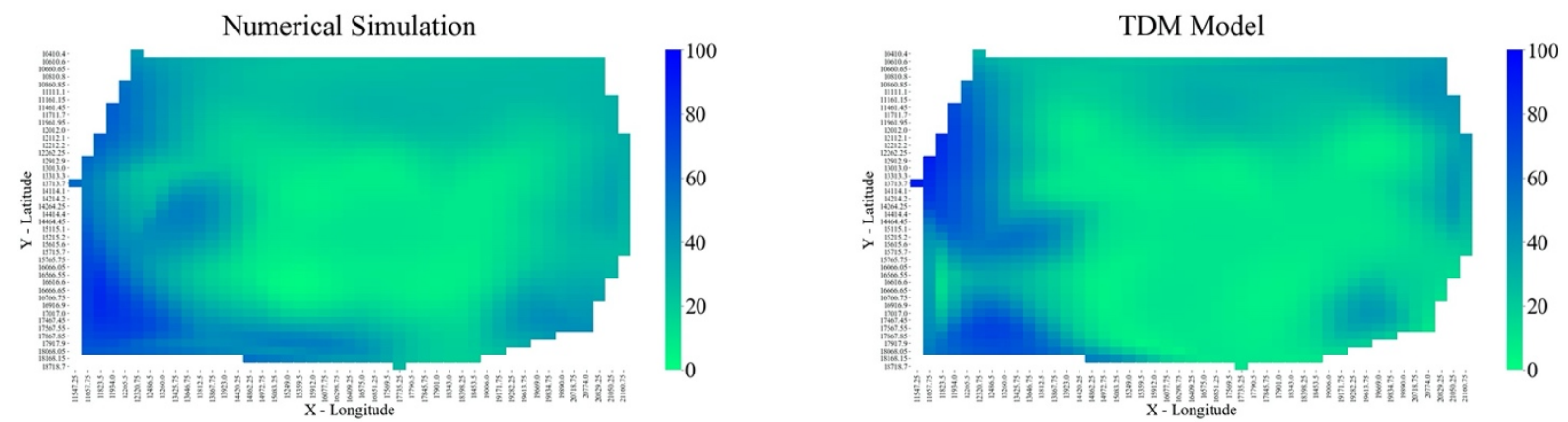

Water Saturation (\%) at 2015-07-31
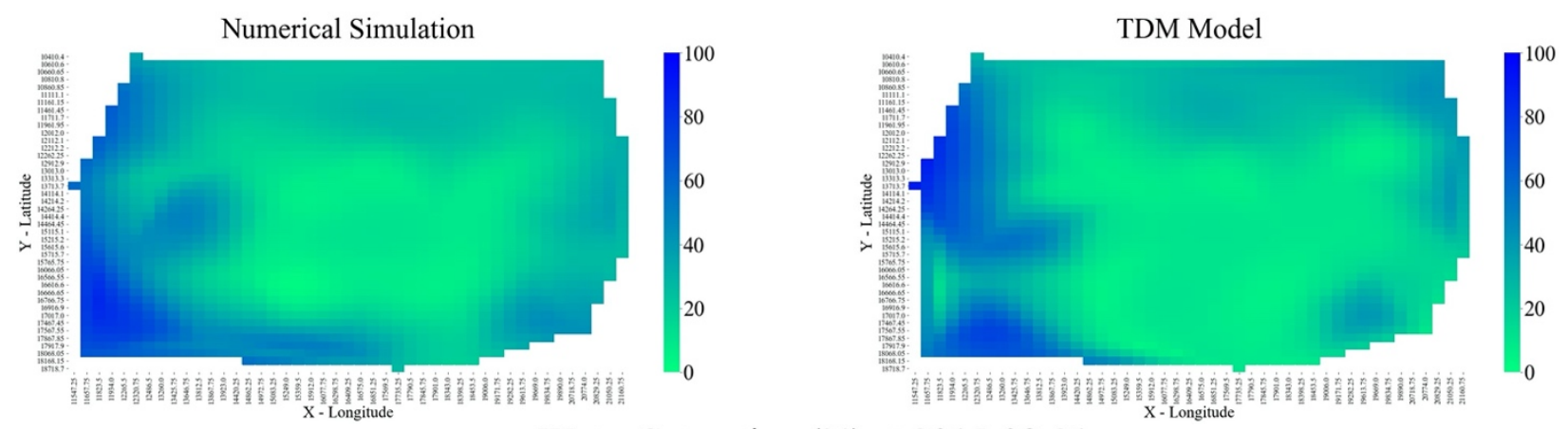

Water Saturation (\%) at 2015-08-31
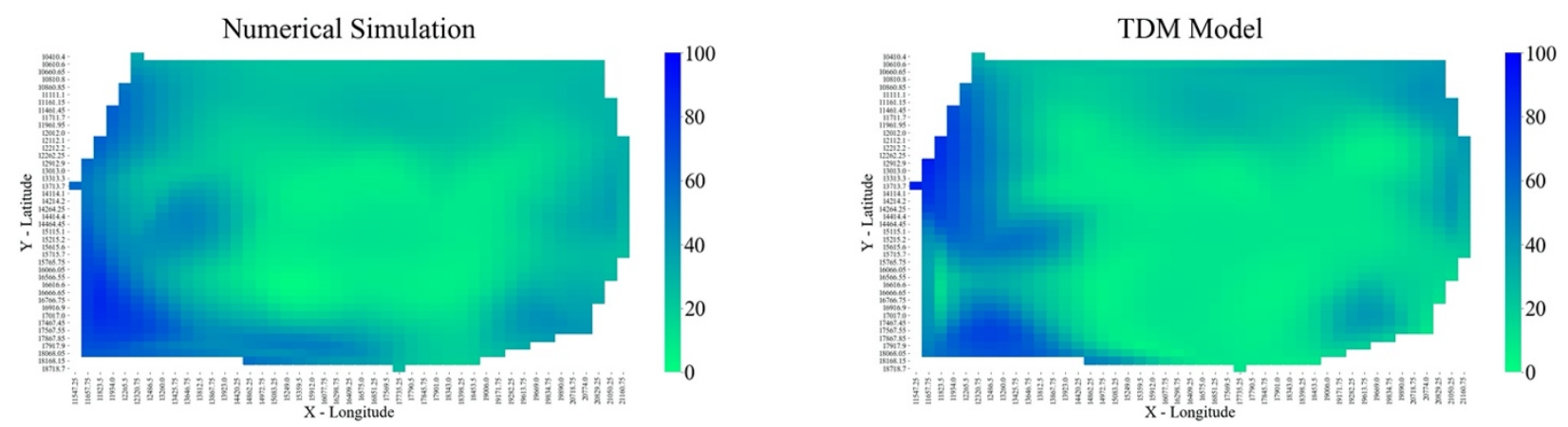
Water Saturation (\%) at 2015-09-30
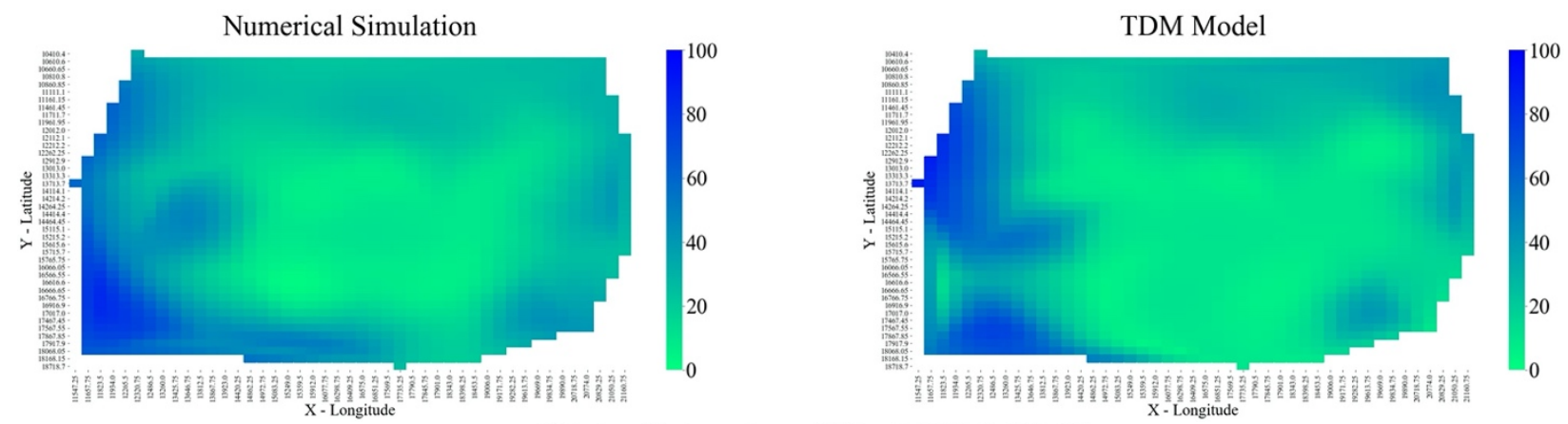

Water Saturation (\%) at 2015-10-31
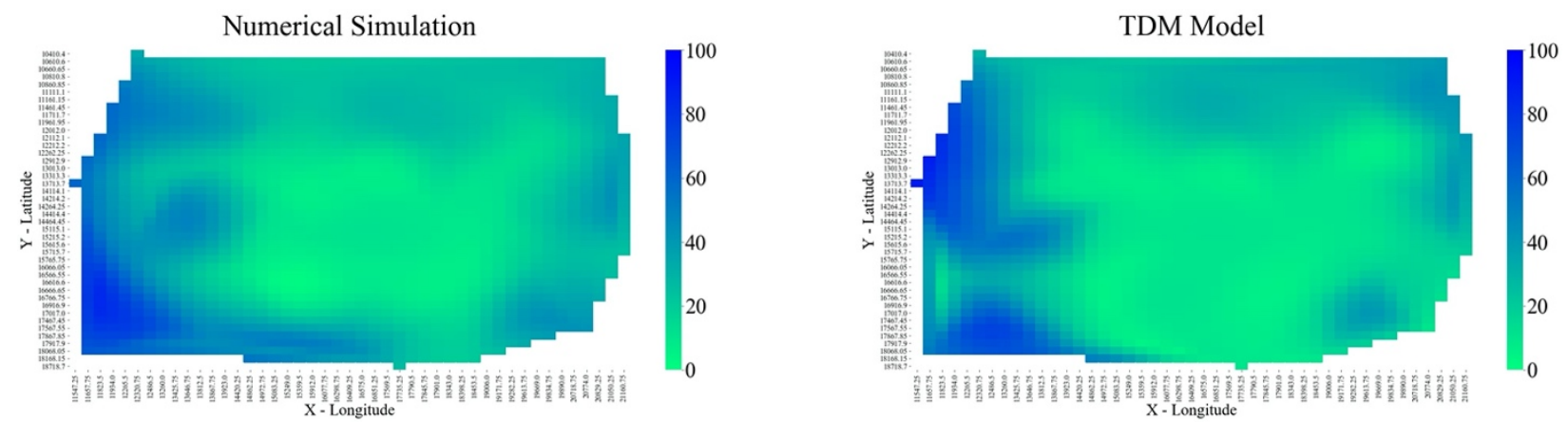

Water Saturation (\%) at 2015-11-30
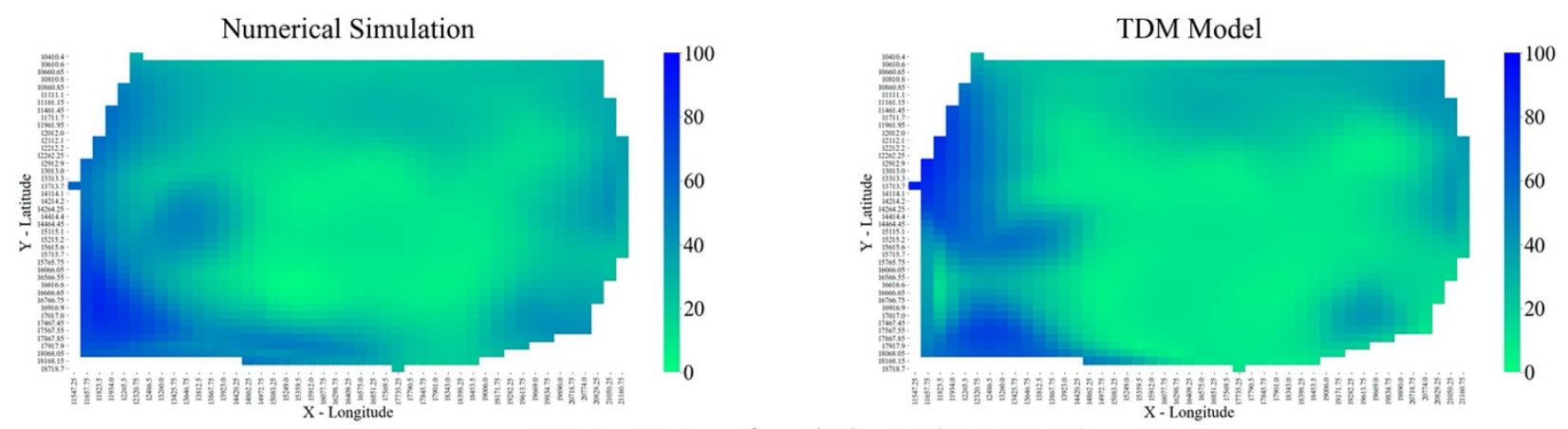

Water Saturation (\%) at 2015-12-31
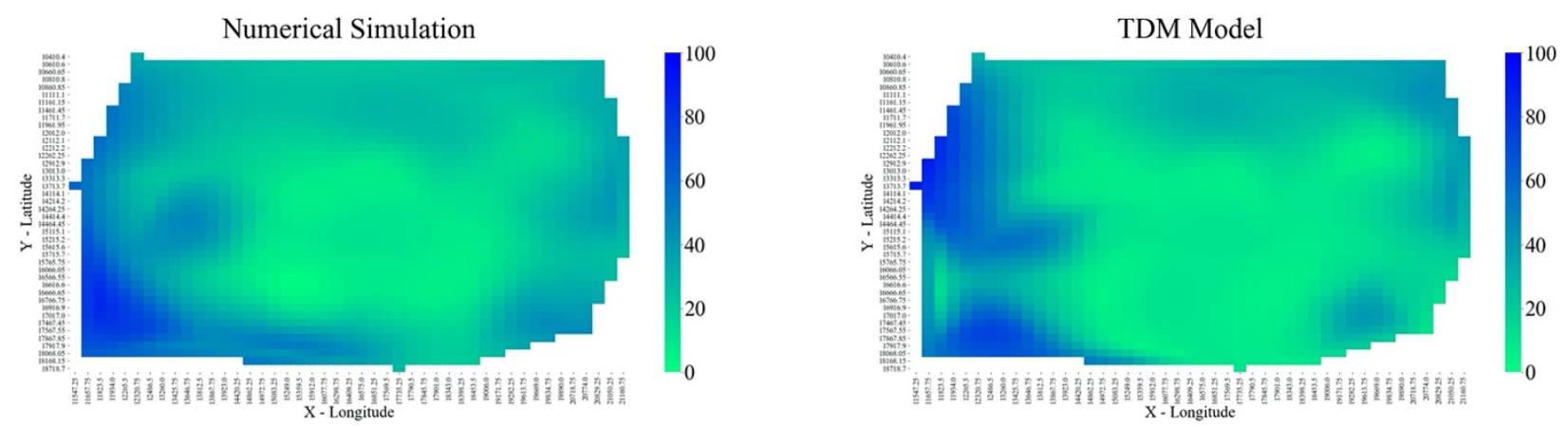
Water Saturation (\%) at 2016-01-31
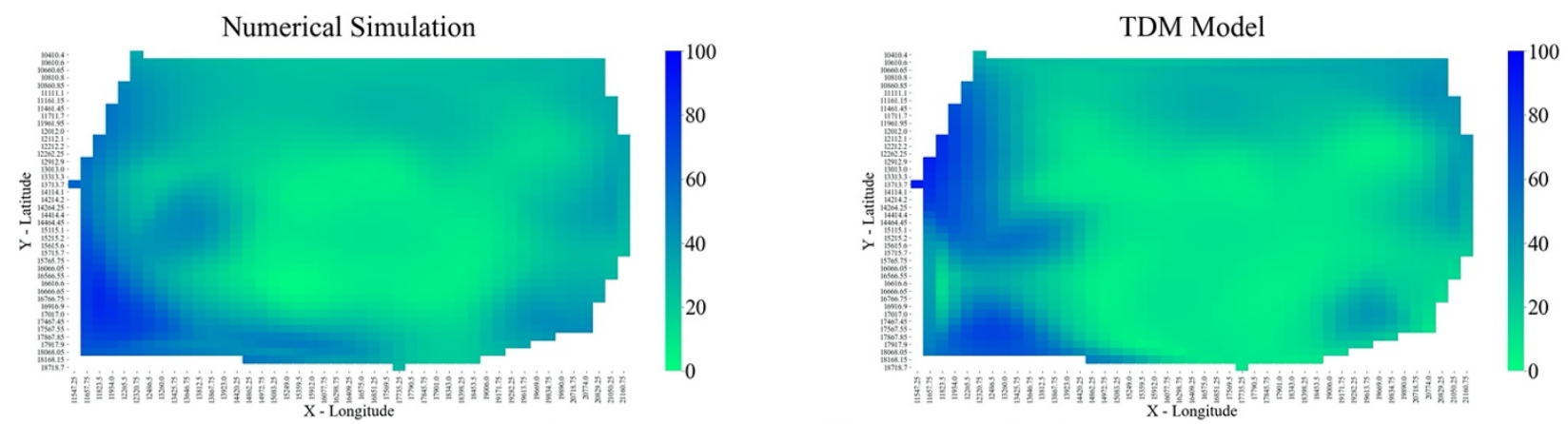

Water Saturation (\%) at 2016-02-29
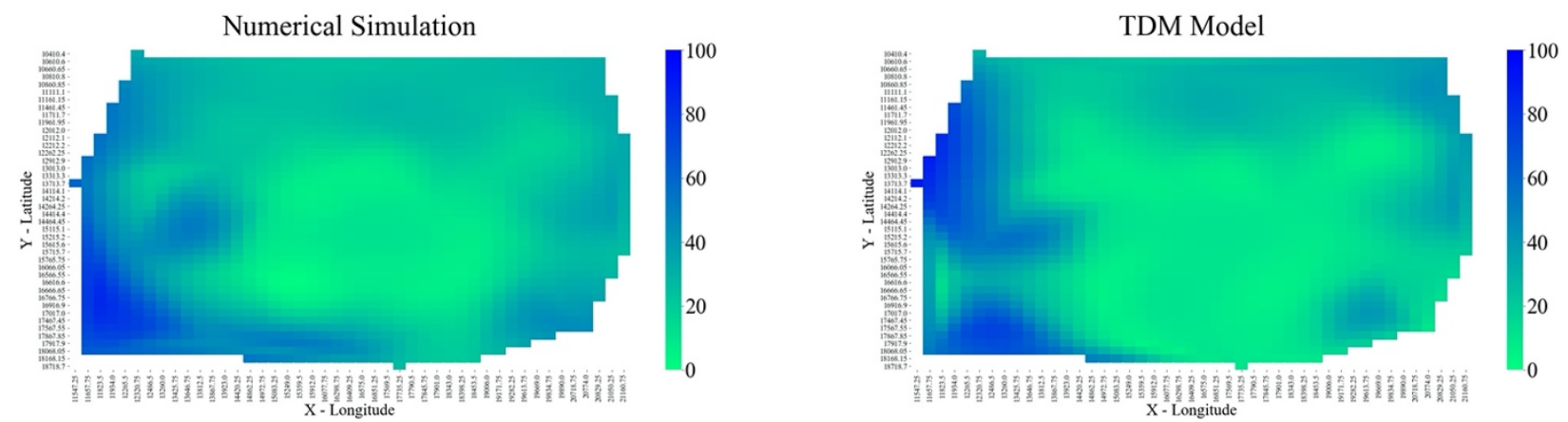

Water Saturation (\%) at 2016-03-31
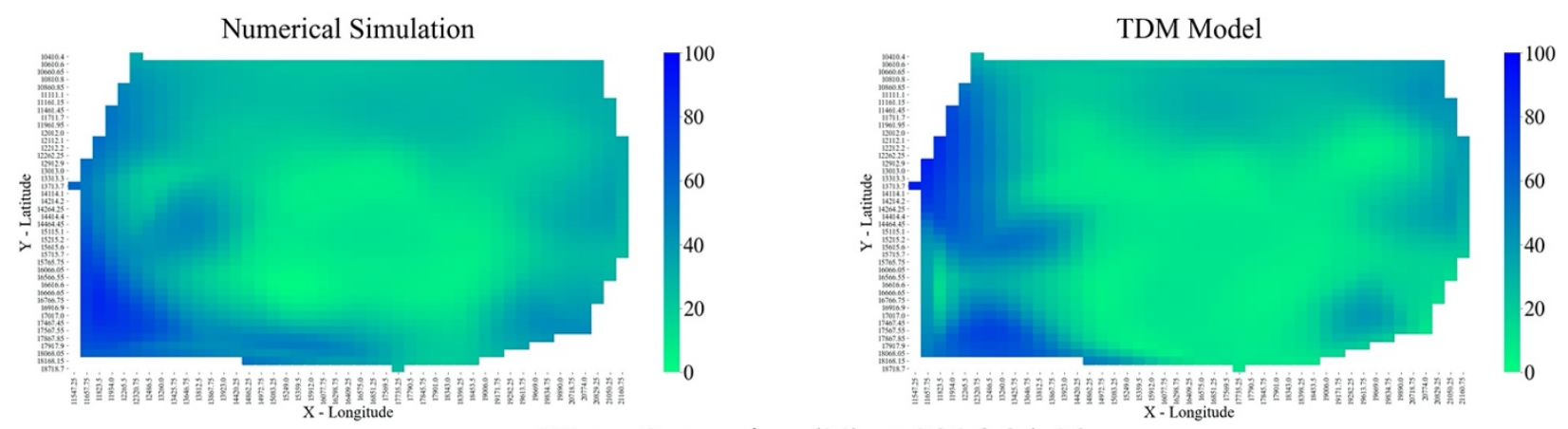

Water Saturation (\%) at 2016-04-30
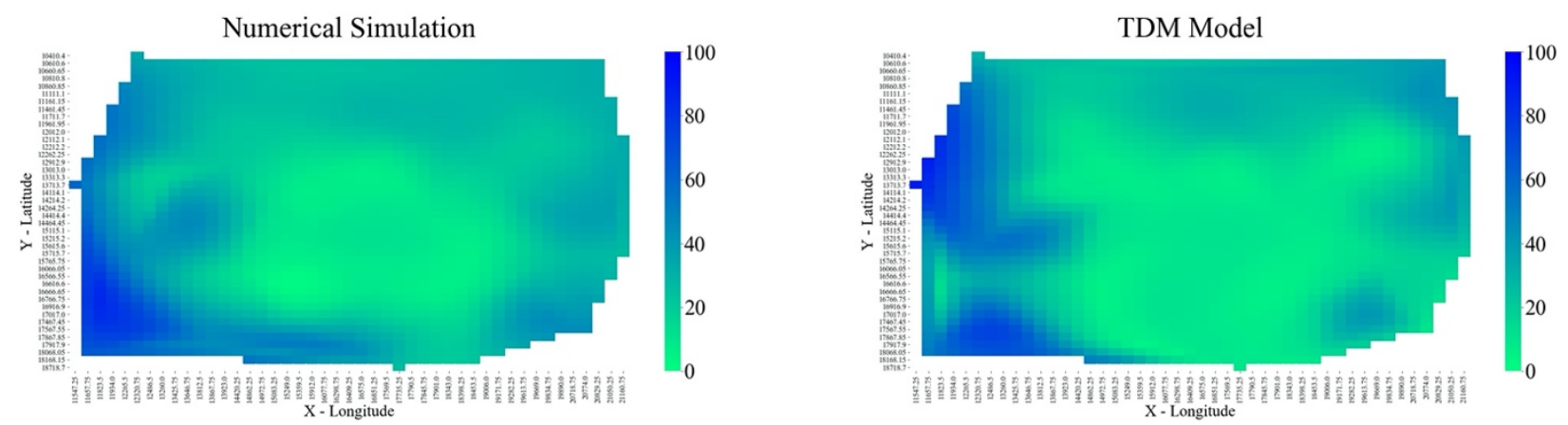
Water Saturation (\%) at 2016-05-31
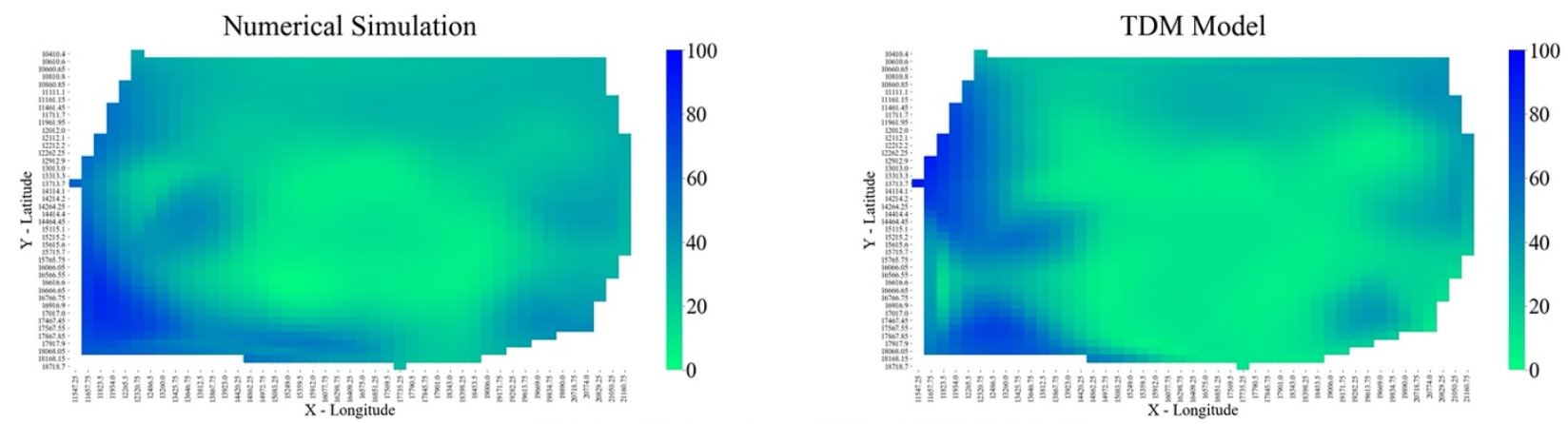

Water Saturation (\%) at 2016-06-30
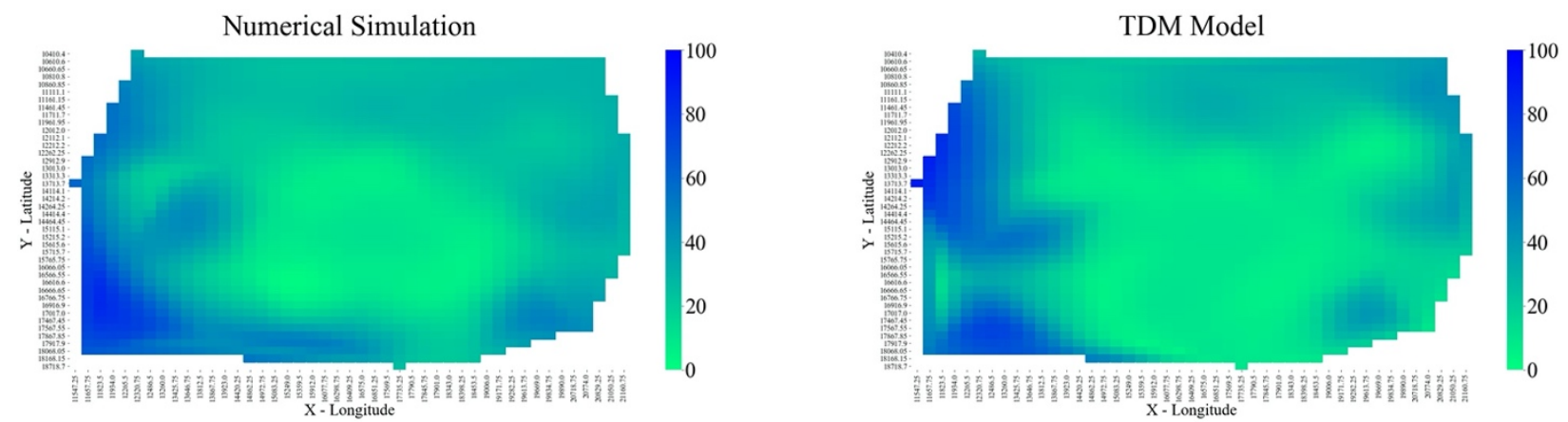

Water Saturation (\%) at 2016-07-31
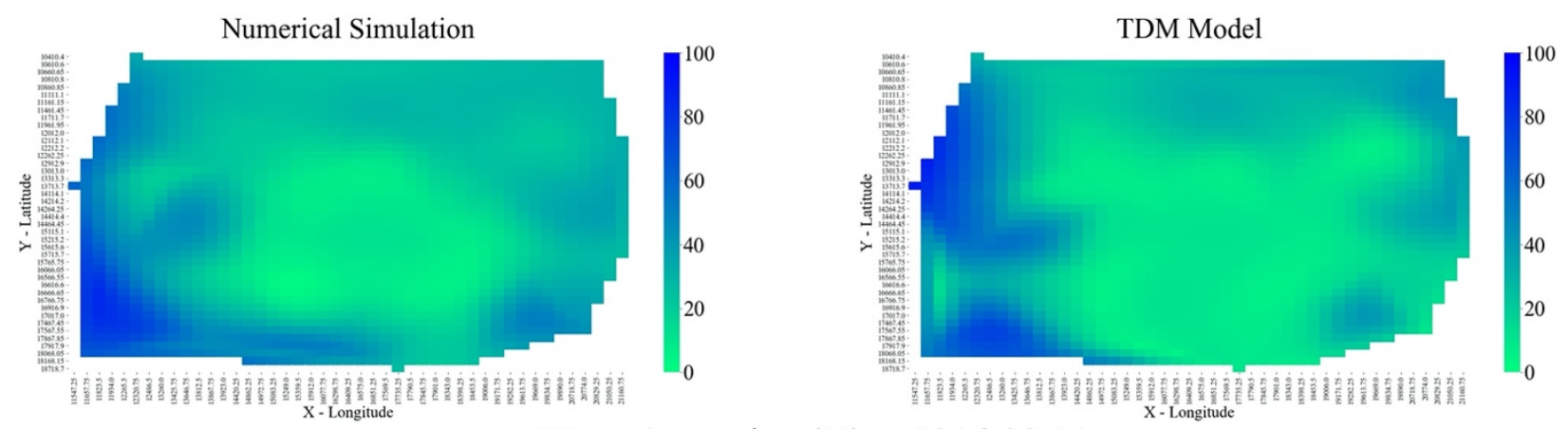

Water Saturation (\%) at 2016-08-31
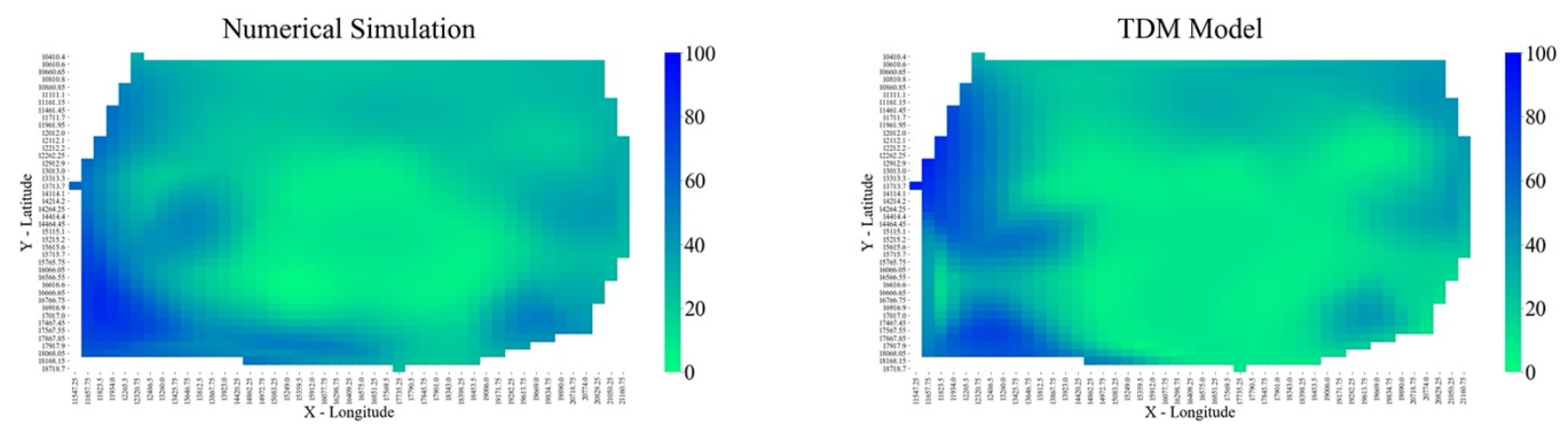
Water Saturation (\%) at 2016-09-30
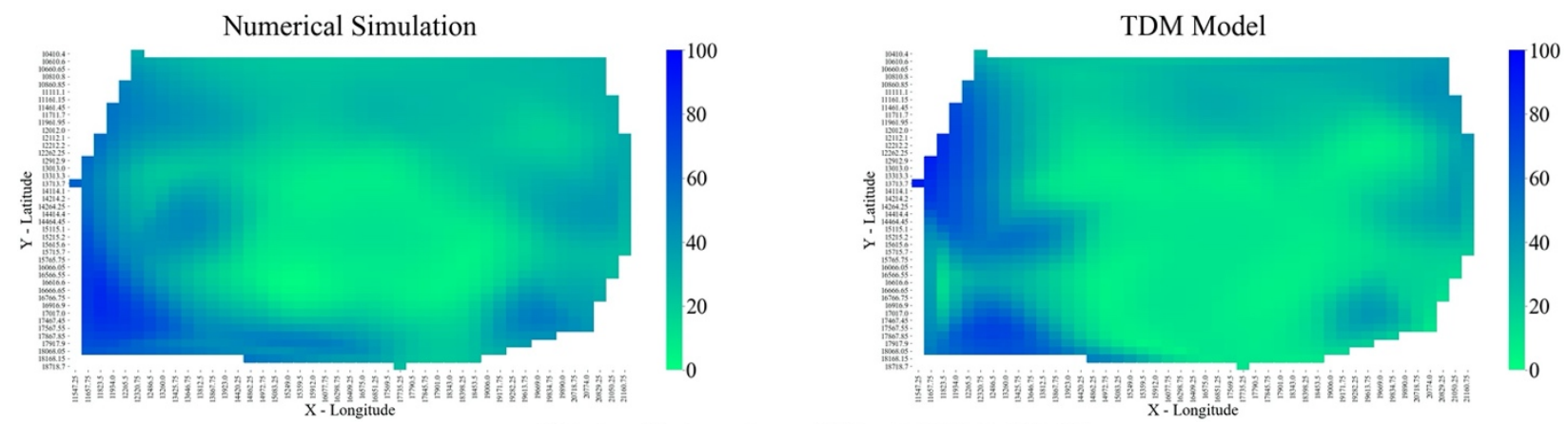

Water Saturation (\%) at 2016-10-31
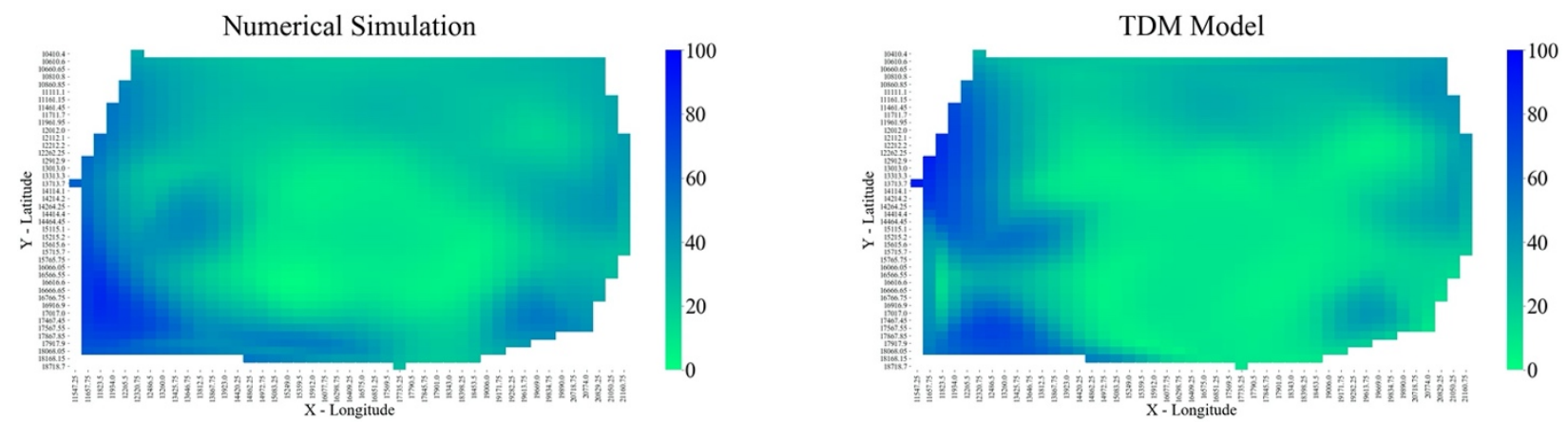

Water Saturation (\%) at 2016-11-30
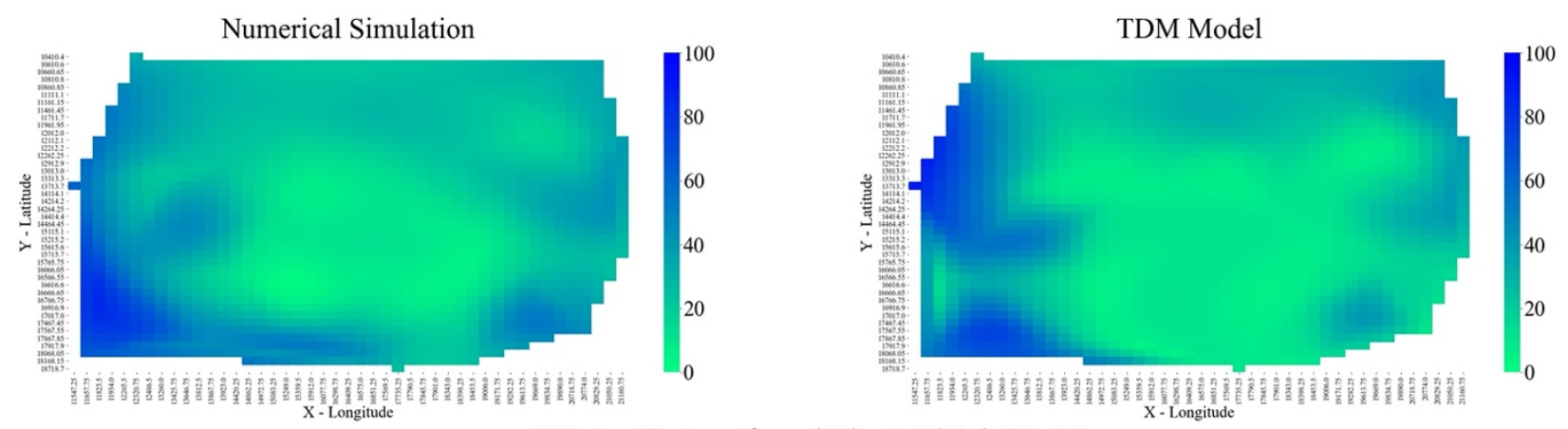

Water Saturation (\%) at 2016-12-31
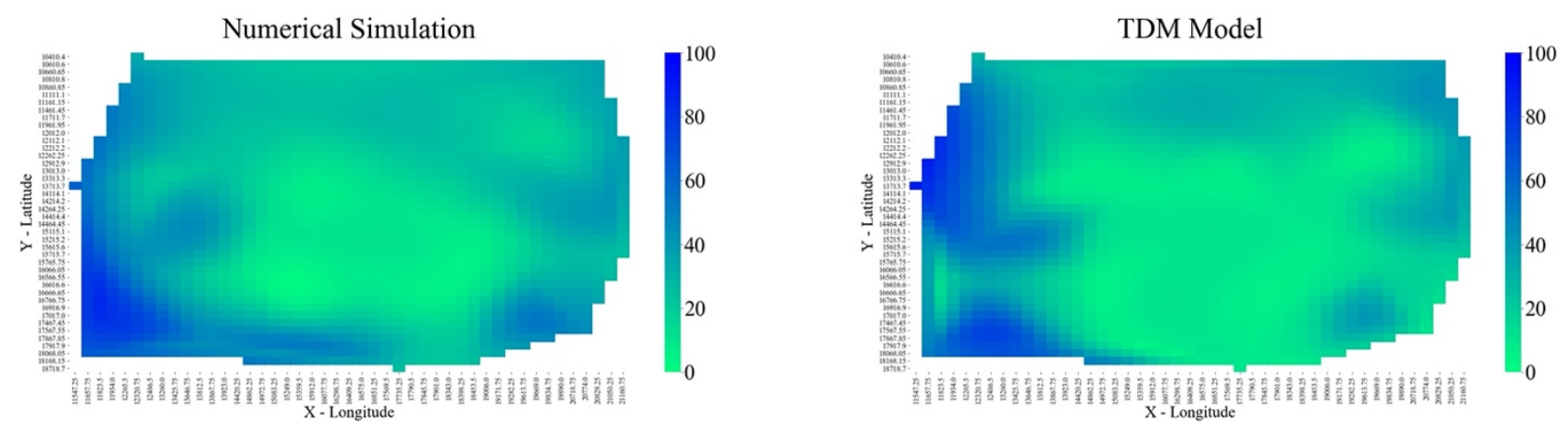